\title{
Reticulados de conceitos
}

\author{
Alexandre Luiz Junqueira Hadura Albano
}

\author{
DISSERTAÇÃO APRESENTADA \\ $\mathrm{AO}$ \\ Instituto de MATEMÁtica E Estatística \\ DA \\ Universidade DE SÃo PAUlo \\ PARA \\ OBTENÇÃ O DO TÍTULO \\ $\mathrm{DE}$ \\ Mestre EM CiÊnCIAS
}

\author{
Programa: Ciência da Computação \\ Orientador: Prof. Dr. Alair Pereira do Lago
}

Durante o desenvolvimento deste trabalho, o autor recebeu auxílio financeiro do CNPq através do processo 132590/2009-3.

São Paulo, dezembro de 2011 


\section{Reticulados de conceitos}

Esta dissertação contém as correções e alterações sugeridas pela Comissão Julgadora durante a defesa realizada por Alexandre Luiz Junqueira Hadura Albano em 02/12/2011.

O original encontra-se disponível no Instituto de Matemática e Estatística da Universidade de São Paulo.

Comissão Julgadora:

- Prof. Dr. Alair Pereira do Lago - IME-USP

- Prof. Dr. Carlos Eduardo Ferreira - IME-USP

- Prof. Dr. Renato José da Silva Carmo - UFPR 


\section{Agradecimentos}

Seguramente este trabalho não existiria sem o apoio que recebi de minha família e amigos. Por estas pessoas, fui efetivamente apoiado e quero agradecer imensamente. Por ser metódico, agradecerei em especial a cada amigo em frentes. Agradeço a meus amigos do bairro: Daniel Neiva, Guilherme, Ricardo e Rodrigo Manrubia, Pitão, Rica, Relva, Ruyzão (ceará) e Sapo. Com a mesma magnitude, agradeço aos amigos do BCC (Adroaldo, Cosen, Gigante, Hugo, Marciô Oshiro, Mariana, Pedro Simões, [Rafael], Lobaaaaaaato e Rodolpho Atoji). Agradeço da mesma forma aos amigos do NUMEC (Álvaro, Alexandre Chegado Freire, Guilherme Mota, Guilherme Puglia, Rafael Barbosa, Rafael Schouery e Roberto Parente). Foi sensacional trabalhar ao lado de vocês. Também agradeço a outros colegas do IME, como Danilo Castro e outros, com os quais convivi e muitas vezes neles me inspirei.

Agradeço aos professores do IME-USP, em especial, aos docentes do grupo de Otimização Combinatória. Sem intenções bajulatórias, quero agradecer primeiramente ao meu orientador, Prof. Alair, que sempre acreditou em minha capacidade e se preocupou em me expor diversas áreas do conhecimento, para que eu pudesse escolher alguma na qual trabalhar. Aos professores Carlinhos, Yoshiko, Cris e Coelho, por ministrarem aulas com grande clareza, excelente didática e esforço.

Por último, quero agradecer em especial à minha família (em particular, o paitrocínio que recebo) e à minha menina linda m.a.gz :). 



\section{Resumo}

A Análise de Conceitos Formais (FCA) é uma teoria matemática que formaliza a noção de conceitos e hierarquias conceituais. De importância central a esta teoria é uma estrutura algébrica denominada reticulado de conceitos. Esta estrutura é definida em função de um conjunto de objetos, outro de atributos e uma relação que indica os atributos apresentados por cada objeto.

Uma representação gráfica de um reticulado de conceitos, por meio de uma interface computacional, é capaz de expor regularidades presentes em dados a um usuário, e este pode então realizar tarefas de análise exploratória de dados. Este tipo de aplicação de FCA vem sendo empregado em dezenas de projetos pertencentes a áreas diversas, como medicina, serviços de inteligência, engenharia de software e bioinformática.

Mostramos neste trabalho um sistema de análise exploratória de dados baseado em FCA, e sua utilização sobre dados reais. Também é mostrado como reticulados de conceitos podem ser empregados em interfaces de recuperação de informação.

Do ponto de vista algorítmico, analisamos métodos computacionais para a determinação do reticulado de conceitos, e também de uma subestrutura simplificada, o conjunto de conceitos.

O tamanho de um reticulado de conceitos pode ser exponencial em função dos tamanhos dos conjuntos de objetos e de atributos. Assim, é de vital interesse o estabelecimento de cotas superiores para o número de conceitos de um reticulado. Neste trabalho, apresentamos as cotas já conhecidas presentes na literatura. Também estabelecemos uma nova cota superior, e mostramos famílias de casos em que nossa cota superior é mais justa que as demais. Para algumas famílias particulares, nossa cota é polinomial, enquanto que as demais são exponenciais.

Palavras-chave: Análise de Conceitos Formais, Reticulados de conceitos, Sistemas conceituais de informação, Bicliques maximais. 



\section{Abstract}

Formal Concept Analysis (FCA) is a mathematical theory that formalizes the notion of concepts and conceptual hierarchies. Of central importance to this theory is an algebraic structure termed concept lattice. Such structure becomes defined after being given one set of objects, one of attributes, and an incidence relation describing the attributes held by each object.

A graphical representation of a concept lattice, by means of a computational interface, is capable of unfolding regularities present in data to an user, who is then able to conduct exploratory data analysis tasks. This sort of FCA application is currently deployed in tens of projects belonging to a wide range of areas, such as medicine, intelligence services, software engineering and bioinformatics.

We show in this work an FCA-based system of exploratory data analysis, and its use over real data. Moreover, it is shown how concept lattices can be employed in information retrieval interfaces.

From the algorithmic viewpoint, we analyse computational methods for the determination of a concept lattice, and also of a simplified substructure, the concept set.

The size of a concept lattice can be exponential when compared to the size of the objects and the attributes sets. Therefore, it is of paramount interest the establishment of upper bounds for the number of concepts of a lattice. In this work, we present the upper bounds already known in the literature. We also establish a new upper bound, and show families of cases in which our bound is sharper than the others. For particular families, our bound is polynomial, whereas the other bounds are exponential.

Keywords: Formal Concept Analysis, Concept lattices, Conceptual information systems, Maximal bicliques. 



\section{Sumário}

1 Introdução 15

2 Fundamentos de FCA 19

2.1 Definições e propriedades básicas . . . . . . . . . . . . . . . . . . . . 19

2.2 Operadores de Derivação . . . . . . . . . . . . . . . . . . . . . . . . . . . . . . . . . . . . . . . . . . . . . . . .

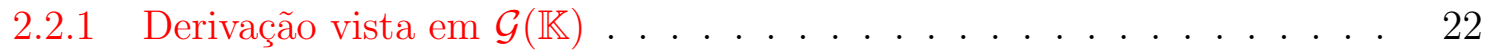

2.2 .2 Bicliques de $\mathcal{G}(\mathbb{K}) \ldots \ldots \ldots \ldots$. . . . . . . . . . . . . . . . . . . . . . . . . . . . . . . .

2.3 Conceitos formais . . . . . . . . . . . . . . . . . . . . . . . . . . . . . . . . . .

2.4 Hierarquias de conceitos . . . . . . . . . . . . . . . . . . . . . . . . . . . . . . .

2.5 Um pouco de teoria de ordem e de reticulados . . . . . . . . . . . . . . . . . 30

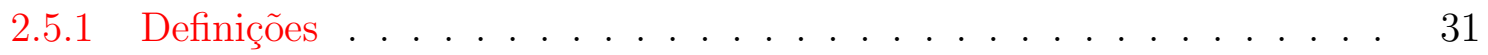

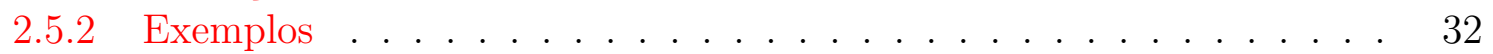

2.5.3 Reticulados completos e famílias . . . . . . . . . . . . . . . 33

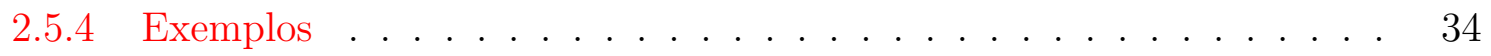

2.5.5 Preservação de ordem e homomorfismos . . . . . . . . . . . . . . . 34

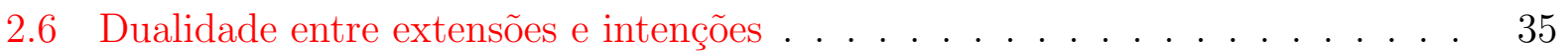

2.7 Teorema fundamental . . . . . . . . . . . . . . . . 36

3 O problema da contagem de conceitos $4 \mathbf{4 5}$

3.1 Problemas de contagem . . . . . . . . . . . . . . . . . 45

4 Aplicações $\quad 51$

4.1 Sistemas conceituais de informação . . . . . . . . . . . . . . . . . 51

4.1.1 Fase de criação de reticulados de um sistema . . . . . . . . . . . . 51

4.1 .2 Fase de análise dos dados . . . . . . . . . . . . . . . . . . . . . . . . . . . . . . . . . 55

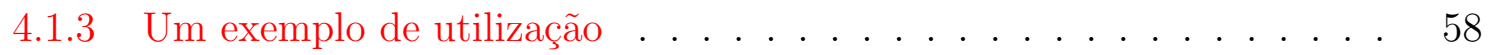

4.1.4 Sistemas Conceituais de Informação implantados . . . . . . . . . . . 61

4.2 FCA em recuperação de informação . . . . . . . . . . . . . . . . . . 62

5 Determinação do conjunto de conceitos $\quad \mathbf{6 7}$

5.1 Fecho por intersecções de intenções-objetos . . . . . . . . . . . . . . . . . . 67

5.1 .1 Análise de Complexidade . . . . . . . . . . . . . . . 71

5.2 Algoritmo de Ganter . . . . . . . . . . . . . . . . . . . . . 74

5.2.1 Análise de complexidade . . . . . . . . . . . . . . . . . 79

5.2 .2 Adaptação para a determinação dos conceitos . . . . . . . . . . . . 79 
6 Determinação do reticulado de conceitos $\quad 81$

6.1 Algoritmo dos Próximos Vizinhos . . . . . . . . . . . . . . . . . . . . . 81

6.1.1 Análise de complexidade . . . . . . . . . . . . . . . . . 85

6.2 Algoritmo de Nourine e Raynaud . . . . . . . . . . . . . . . . . . . . . 86

6.2.1 Árvore Lexicográfica . . . . . . . . . . . . . . . . . . . 91

6.2.2 Estrutura utilizada para representação da árvore lexicográfica . . . . 92

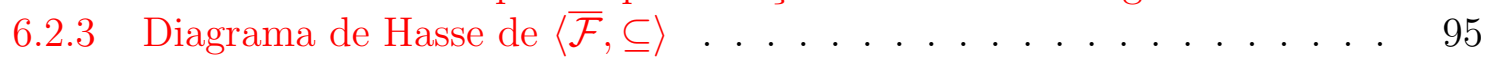

6.2.4 Redução a partir do problema de construção de reticulado de conceitos 98

7 Contextos com número polinomial de conceitos 101

7.1 Definições e a cota da classe mínima . . . . . . . . . . . . . . . . . . . 102

7.2 As cotas dos graus máximos . . . . . . . . . . . . . . . . . . . . . . . . . . . . . . . . . . . . . . . . . . .

7.3 Família esparsa com $|\mathfrak{B}|$ superpolinomial . . . . . . . . . . . . . . . . 105

7.4 A arboricidade de um grafo . . . . . . . . . . . . . . . . . . . . . . . . . . . . . . . . . . . . . . . . .

7.5 A cota da arboricidade . . . . . . . . . . . . . . . . 108

7.6 Cota inferior para o resultado de Eppstein . . . . . . . . . . . . . . . . . 112

7.7 A cota do número de arestas . . . . . . . . . . . . . . . . . . . . . . . . . . . . . . . . . . . . .

7.8 A cota da convexidade . . . . . . . . . . . . . . . . . . . . . . . . . . . . . . . . . . . . . . . . . .

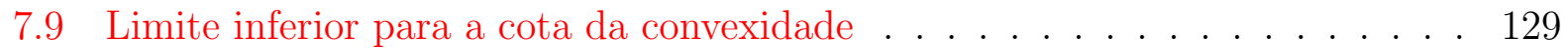

7.10 Complexidade do cálculo de convexidade . . . . . . . . . . . . . . . 134

8 Conclusão e trabalhos futuros $\quad 137$ 


\section{Lista de Figuras}

1.1 Reticulado associado ao contexto da Tabela 1.1. Rótulos inferiores estão associados aos animais, enquanto que rótulos superiores estão associados aos seus

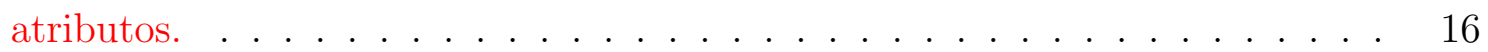

2.1 Representação por grafo bipartido do subcontexto dos vertebrados. . . . . . 20

2.2 Representações por tabela e grafo bipartido de um contexto. . . . . . . . . . 20

2.3 Derivações de conjuntos de objetos e de atributos, quando vistas em $\mathcal{G}(\mathbb{K})$. . $\quad 23$

2.4 Duas bicliques maximais em $\mathcal{G}(\mathbb{K})$ representando conceitos. . . . . . . . . . 26

2.5 Hierarquia de conceitos com rotulação cheia. . . . . . . . . . . . . . . . . 28

2.6 Hierarquia de conceitos com rotulação reduzida. . . . . . . . . . . . . . . . . 29

2.7 Recuperar extensão: toma-se rótulos abaixo de conceitos no interior da elipse. 30

2.8 Recuperar intenção: toma-se rótulos acima de conceitos no interior da elipse. 30

2.9 Diagramas de Hasse. . . . . . . . . . . . . . . . . . . . . . . . . . 31

2.10 Representações de ordens parciais. . . . . . . . . . . . . . . 33

2.11 Reticulados de extensões e de intenções do subcontexto dos vertebrados. . . 36

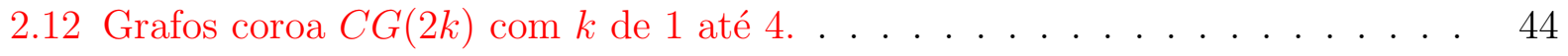

4.1 Reticulado de conceitos do contexto formal sobre opcionais. . . . . . . . . 53

4.2 Reticulado de conceitos de uma escala interordinal, sobre kms rodados. . . . 54

4.3 Reticulado de conceitos de uma escala interordinal, sobre preços. . . . . . . . 54

4.4 Análise do conjunto de carros sob o aspecto "opcionais". . . . . . . . . . . 56

4.5 Reticulado sobre preços com foco no conceito de carros com preço entre

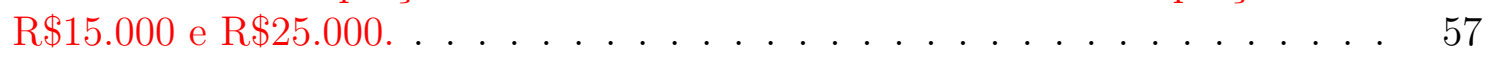

4.6 Reticulado sobre preços com zoom sobre carros com direção hidráulica e ar condicionado. . . . . . . . . . . . . . . . . . . 58

4.7 Reticulado sobre preços com foco em objetos (carros) com preço $<22500$. . 59

4.8 Reticulado sobre opcionais e motorização com zoom "preço < 22500" . . . . . 59

4.9 Reticulado sobre opcionais e motorização com zoom "preço < 22500" e foco. 60

4.10 Reticulado sobre realizadores de venda dos carros, após dois zooms. . . . . . 61

4.11 Relatório exibindo três carros. . . . . . . . . . . . . . . . . . . . 61

4.12 Interface convencional de sistema de recuperação de informação. . . . . . . . 63

4.13 Interface de sistema de recuperação de informação com reticulado de conceitos. Após o usuário clicar no vértice associado à palavra-chave "Algoritmos", os sete documentos da lista que apresentam a palavra-chave "Algoritmos" passam a ficar destacados em azul e negrito. . . . . . . . . . . . . 
5.1 Conjuntos de objetos e de atributos, ordenados, como exemplo de entrada. . 70

5.2 Uma árvore de prefixos, com três vértices destacados, e duas de suas rotulações. 72

5.3 Uma árvore de prefixos de uma família de subconjuntos e as rotulações de quatro de seus vértices. . . . . . . . . . . . . . . 73

6.1 Conceito do contra-exemplo para a recíproca da Proposição 6.1. . . . . . . 83

6.2 Contra-exemplo para a recíproca da Proposição 6.1. . . . . . . . . . . . 83

6.3 Diagrama de Hasse do fecho por uniões (6.2), juntamente com os valores da função $\gamma \ldots \ldots \ldots \ldots$. . . . . . . . . . . . . . . . . . . . . 87

6.4 Árvore lexicográfica do fecho por uniões $\overline{\mathcal{F}}$ descrito em (6.2) . . . . . . . . . 92

6.5 Árvore lexicográfica da familia $\overline{\mathcal{F}}$, descrita em (6.2), incluindo os valores de $\gamma .93$

7.1 Família de grafos com grau máximo elevado, muitas bicliques com arestas, mas poucas bicliques maximais. . . . . . . . . . . . . . . . . . . 104

7.2 Família de grafos com $\Delta_{U}$ e $\Delta_{W}$ em $\Omega(n) . \ldots \ldots$. . . . . . . . . . . . . 105

7.3 Família esparsa de grafos com $\Omega\left(\sqrt{2}^{\sqrt{n}}\right)$ bicliques maximais. . . . . . . . . 106

7.4 Uma 2-floresta-decomposição de um membro da família descrita na Figura 7.2.107

7.5 Um grafo com um subgrafo bipartido completo maximal destacado. . . . . . 109

7.6 Família Ferrers(2k), cujos membros são imunes à Proposição 7.14. . . . . . . 116

7.7 Vértices de $U$ com vizinhança convexa sob diferentes bijeções de $W$. . . . . 118

7.8 Diferença arbitrária entre a convexidade sobre $W$ e sobre $U$. . . . . . . . . . 120

7.9 Um grafo Ferrers-coroa conexo, com vizinhanças distintas, $\left|U_{F}\right|=3\left(=\left|W_{F}\right|\right)$

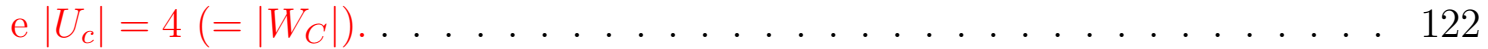

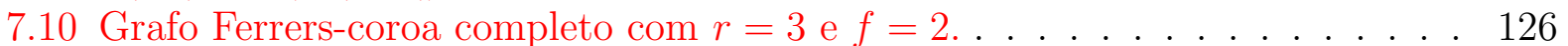

7.11 Grafos interordinais de ordem 1 até $6, \operatorname{com} l=\left\lceil\frac{k}{2}\right\rceil \ldots . . . . .131$ 


\section{Lista de Tabelas}

1.1 Um exemplo de contexto formal. . . . . . . . . . . . . . . . . . 15

2.1 Subcontexto dos vertebrados. . . . . . . . . . . . . . . . . . . . 19

4.1 Tabela de carros . . . . . . . . . . . . . . . . . . . . 51

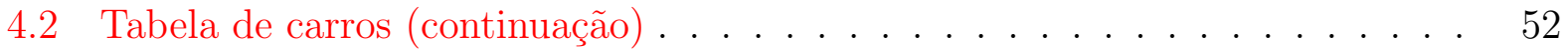

4.3 Dados sobre opcionais dos carros. . . . . . . . . . . . . . . . . . . 52

4.4 Contexto formal sobre opcionais dos carros. . . . . . . . . . . . . . 52

4.5 Contexto formal obtido após definição de escala interordinal. . . . . . . . . . 53

4.6 Contexto formal sobre combustível dos carros. . . . . . . . . . . . . . 55

5.1 Exemplo de execução do Algoritmo 2. . . . . . . . . . . . . . . . . . . . 79 



\section{Capítulo 1}

\section{Introdução}

No início da década de 1980, um grupo da Universidade de Darmstadt iniciou atividades visando "reestruturar a teoria de ordem matemática e a teoria dos reticulados". Conforme o artigo seminal de Wille [Wil82], tal reestruturação da teoria dos reticulados consistia em uma "tentativa de revigorar conexões com a cultura geral, ao interpretar a teoria [dos reticulados] da maneira mais concreta possível, a fim de promover melhor comunicação entre os teóricos de reticulados e potenciais usuários da teoria dos reticulados.".

Surgia então a Análise de Conceitos Formais (Formal Concept Analysis), uma teoria matemática baseada na formalização de conceitos e hierarquias de conceitos. Em FCA, o conjunto de conceitos e sua hierarquia ficam definidos após ser dada uma unidade básica de informação: o contexto formal. Esta unidade básica consiste num conjunto de objetos, outro de atributos e uma relação entre eles, de forma a indicar quais objetos apresentam quais atributos.

Muitas vezes diremos apenas "contexto" para nos referirmos a um contexto formal. Vemos na Tabela 1.1, retirada de [CR04], um contexto em que o conjunto de objetos é formado por sete animais, que podem ou não possuir cada um dos nove atributos designados nas colunas da tabela, conforme assinalado por um ' $X$ '.

\begin{tabular}{|c|c|c|c|c|c|c|c|c|c|}
\hline & 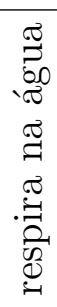 & $\begin{array}{l}\tilde{p} \\
\stackrel{p}{2}\end{array}$ & $\begin{array}{l}\stackrel{0}{0} \\
.0 \\
.3 \\
0 \\
0 \\
0 \\
0\end{array}$ & 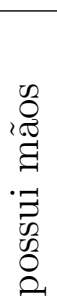 & 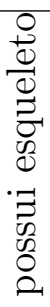 & 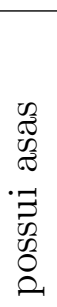 & 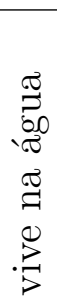 & 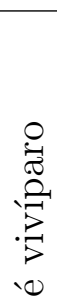 & 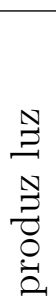 \\
\hline morcego & & $x$ & & & $\times$ & $x$ & & $x$ & \\
\hline águia & & $x$ & $x$ & & $x$ & $x$ & & & \\
\hline macaco & & & & $x$ & $x$ & & & $x$ & \\
\hline peixe papagaio & $x$ & & $x$ & & $x$ & & $x$ & & \\
\hline pinguim & & & $x$ & & $x$ & $x$ & $x$ & & \\
\hline tubarão & $\times$ & & & & $\times$ & & $\times$ & & \\
\hline peixe lanterna & $x$ & & & & $\times$ & & $x$ & & $x$ \\
\hline
\end{tabular}

Tabela 1.1: Um exemplo de contexto formal. 
A interpretação concreta da teoria dos reticulados, pretendida pelo grupo em Darmstadt, significaria que todo contexto possui um reticulado associado, e reciprocamente, todo reticulado está associado a algum contexto. Neste sentido, a teoria que fora desenvolvida interpreta a subteoria dos reticulados completos: isto é, a classe dos reticulados associados a contextos formais é exatamente a classe dos reticulados completos [Wil82], classe esta que inclui todos os reticulados finitos.

Em particular, o reticulado associado ao contexto representado na Tabela 1.1 está retratado na Figura 1.1:

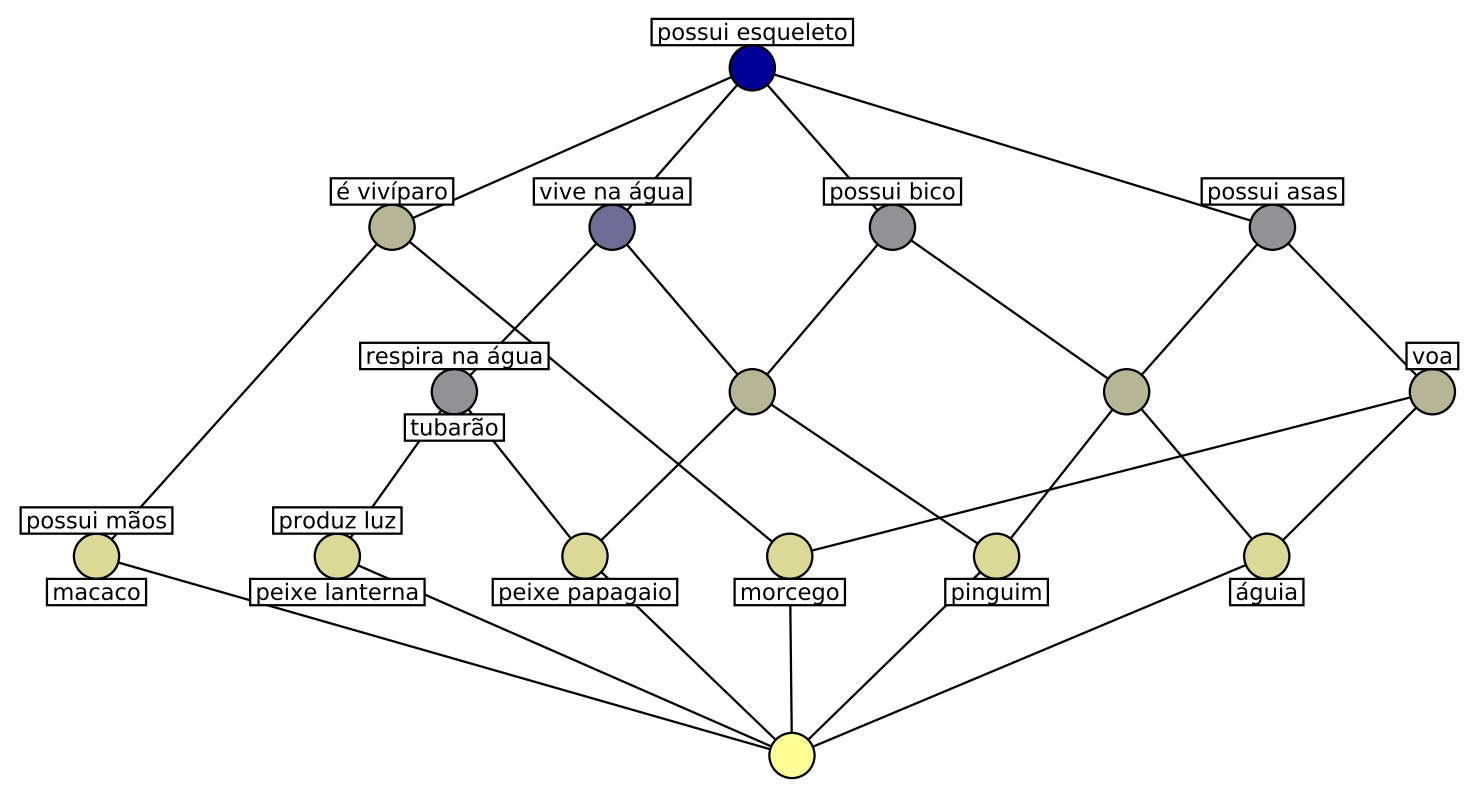

Figura 1.1: Reticulado associado ao contexto da Tabela 1.1. Rótulos inferiores estão associados aos animais, enquanto que rótulos superiores estão associados aos seus atributos.

O desenvolvimento das definições e resultados fundamentais de FCA é normalmente realizado apenas com o emprego da linguagem da teoria dos conjuntos e da álgebra. No entanto, é sabido que a informação presente em um contexto formal pode ser traduzida matematicamente em um grafo bipartido [Sch82], como nos foi comunicado por [Gan10]. Sob esta tradução, objetos matemáticos notáveis de um contexto formal correspondem a objetos matemáticos notáveis de seu grafo bipartido associado. Mostraremos estes desenvolvimentos durante o Capítulo 2. Ademais, mostramos uma família de contextos cujos reticulados crescem exponencialmente em função do tamanho de seus contextos. Os grafos bipartidos associados aos contextos desta família são conhecidos como grafos coroa.

Além dos tamanhos dos reticulados serem eventualmente exponenciais, um resultado devido a Sergei Kuznetsov classifica o problema de contagem de conceitos como difícil [Kuz01]. Isto é, a existência de um algoritmo polinomial que devolve o número de conceitos de um contexto qualquer implica que $\mathbf{P}=\mathbf{N P}$. A exposição deste resultado é realizada no Capítulo 3.

Duas aplicações computacionais baseadas em FCA são expostas durante o Capítulo 4. Especificamente, discorremos acerca dos Sistemas Conceituais de Informação de Becker e Correia [BC04], e exibimos a utilização de um tal sistema sobre dados reais. Neste mesmo 
capítulo é mostrado como a FCA pode ser aplicada a um cenário de recuperação de informação, conforme aplicações sugeridas por Carpineto e Romano [CR04].

O Capítulo 5 contém a exposição e análise de dois algoritmos que são capazes de encontrar o conjunto de conceitos. Este conjunto é exatamente o conjunto dos vértices de reticulados, como os 16 vértices que podem ser vistos na Figura 1.1. O primeiro algoritmo é bastante conhecido dentre a comunidade de FCA e segue quase que diretamente a partir de uma caracterização de conceitos. O segundo é conhecido como algoritmo de Ganter (e também como NextClosure) [Gan84] e apresenta a propriedade de ser um algoritmo de atraso polinomial (polynomial delay). Esta propriedade é desejável, uma vez que o conjunto de conceitos pode ter, conforme destacamos, tamanho exponencial em função do tamanho do contexto.

Durante o Capítulo 6, expomos e analisamos dois algoritmos para o problema de se encontrar o reticulado de conceitos, isto é, seu conjunto de vértices juntamente com seus arcos. Primeiramente expomos a versão do algoritmo de Bordat [Bor86] feita por Carpineto e Romano [CR04], e que é relativamente simples. Em seguida, mostramos o algoritmo de Nourine e Raynaud [NR99], que apresenta na atualidade a menor complexidade computacional conhecida, a saber, $O(|\mathfrak{B}||G||M|)$, onde $\mathfrak{B}$ é o conjunto de conceitos, enquanto que $G$ e $M$ são, respectivamente, os conjuntos de objetos e de atributos.

A família de contextos cujos reticulados crescem exponencialmente, descrita durante o Capítulo 2, serve de motivação para o problema de se estabelecer cotas superiores para $|\mathfrak{B}|$, isto é, para o número de conceitos de um contexto. Para tanto, o desenvolvimento de FCA utilizando a linguagem da teoria dos grafos se mostrará valioso. Em particular, será de grande utilidade a correspondência biunívoca entre os conceitos de um contexto e bicliques maximais do seu grafo bipartido associado.

No Capítulo 7, provamos a validade de todas as cotas superiores para $|\mathfrak{B}|$ encontradas por nós na literatura. Em particular, expomos uma limitação do número de conceitos em função do grau máximo do grafo bipartido associado, e também uma cota superior, devida a David Eppstein [Epp94], em função de uma medida de esparsidade hereditária chamada arboricidade.

Resultados originais A cota superior de David Eppstein é uma limitação do número de bicliques maximais em grafos, e para isto não é necessário que o grafo seja bipartido. Em [Epp94], o autor constrói uma família de grafos, exclusivamente não bipartidos, que servem de limite inferior para sua cota superior. Por não serem bipartidos, tais grafos não servem de limite inferior no nosso cenário. Ademais, entre o limite inferior e a cota superior mostrados por Eppstein, há uma folga de pelo menos $2^{\Upsilon}$, onde $\Upsilon$ é a arboricidade dos grafos da família. Após a exposição da cota de Eppstein, mostramos, neste trabalho, uma família de grafos bipartidos que serve de limite inferior para a cota superior de Eppstein, e que ao mesmo tempo fecha a folga exponencial em $\Upsilon$. Para isso, realizamos um cálculo explícito da arboricidade do grafo coroa, através do teorema de Nash-Williams [NW64].

A cota de Schütt [Sch87], em função do número de arestas, também é exposta. Mostramos ainda como certos argumentos de simplificação podem ser utilizados para limitar o número de bicliques maximais de um grafo em função do número de bicliques maximais de grafos mais simples. 
Ainda no Capítulo 7, demonstramos uma nova cota superior e construímos famílias para as quais nossa cota é a mais justa. Em particular, são construídas famílias para as quais nossa cota é cúbica, enquanto que as demais são exponenciais. Com relação a limites inferiores, construímos uma família infinita, de um parâmetro, para a qual nossa cota é justa assintoticamente. Famílias com maior número de parâmetros também são construídas e suas folgas perante nossa cota superior são calculadas.

A cota que estabelecemos é uma expressão em função de uma medida que chamamos de convexidade, que é definida para qualquer grafo bipartido. Esta medida é naturalmente induzida por uma propriedade bastante conhecida na literatura, a saber, a propriedade de um grafo bipartido ser convexo. Apesar dessa indução natural, não encontramos referências na literatura que definissem esta medida. Ao final do Capítulo 7, demonstramos que o problema de decisão associado ao cálculo desta medida é NP-completo. 


\section{Capítulo 2}

\section{Fundamentos de FCA}

\subsection{Definições e propriedades básicas}

Um contexto formal é uma tripla $\mathbb{K}=(G, M, I)$, onde $G$ é um conjunto cujos elementos são chamados de objetos, $M$ é um conjunto cujos elementos são chamados de atributos ${ }^{1}$ e $I$ é uma relação entre $G$ e $M(I \subseteq G \times M)$, que chamaremos de relação de incidência. Muitas vezes omitiremos o adjetivo formal, dizendo apenas contexto. Quando $|G|$ e $|M|$ forem finitos, é claro que $\mathbb{K}$ pode ser representado por uma tabela, como a Tabela 2.1 a seguir:

\begin{tabular}{|c|c|c|c|c|c|c|c|c|}
\hline & 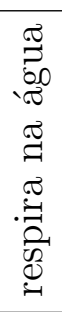 & $\stackrel{\approx}{8}$ & 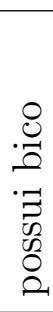 & 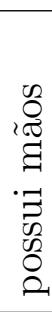 & $\begin{array}{l}0 \\
\stackrel{0}{0} \\
0 \\
\overrightarrow{0} \\
0 \\
0 \\
= \\
0 \\
0 \\
0\end{array}$ & 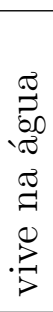 & 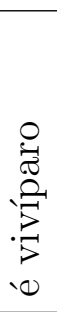 & 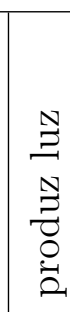 \\
\hline macaco & & & & $x$ & $x$ & & $x$ & \\
\hline peixe papagaio & $x$ & & $x$ & & $x$ & $x$ & & \\
\hline peixe lanterna & $x$ & & & & $x$ & $x$ & & $\times$ \\
\hline águia & & $x$ & $x$ & & $x$ & & & \\
\hline
\end{tabular}

Tabela 2.1: Subcontexto dos vertebrados.

O contexto acima, que será chamado de subcontexto dos vertebrados, foi obtido através da omissão de uma coluna e algumas linhas da Tabela 1.1. Nas aplicações, é frequente que ocorra a disjunção entre o conjunto de objetos e o conjunto de atributos, apesar disto não ser exigido pela definição de contexto formal. Uma representação alternativa de contextos $(G, M, I)$ com $G$ e $M$ disjuntos é um grafo bipartido, como por exemplo o da Figura 2.1, que representa o subcontexto dos vertebrados.

\footnotetext{
${ }^{1}$ Essa escolha de letras é comum na literatura e é fruto de influência da língua alemã: Gegenstände significa "objetos", enquanto que Merkmale significa "atributos".
} 


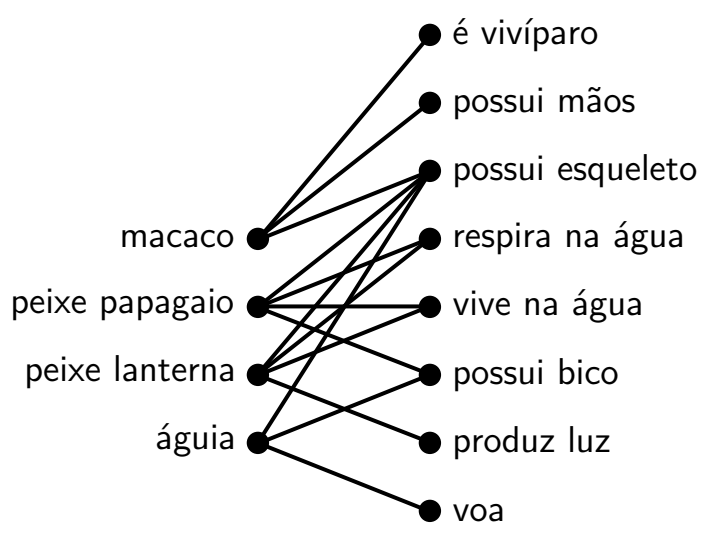

Figura 2.1: Representação por grafo bipartido do subcontexto dos vertebrados.

Se não tivermos a disjunção entre $G$ e $M$, podemos tomar cópias disjuntas de $G$ e de $M$ para representar um contexto $\mathbb{K}$ através de um grafo bipartido. Formalmente, dados $g^{*}$ e $m^{*}$ elementos distintos e dado $\mathbb{K}=(G, M, I)$, defina $\dot{G}=G \times\left\{g^{*}\right\}$ e $\dot{M}=M \times\left\{m^{*}\right\}$. Observe que $\dot{G} \cap \dot{M}=\emptyset$. Além disso, cada $g \in G$ está naturalmente identificado com o elemento $\left(g, g^{*}\right)$ de $\dot{G}$, e o mesmo ocorre para $m \in M$, que se identifica com o elemento $\left(m, m^{*}\right)$ de $\dot{M}$. Em um tratamento mais rigoroso, as bijeções ' $: G \rightarrow \dot{G} \mathrm{e}^{\cdot}: M \rightarrow \dot{M}$ merecem dois símbolos distintos. Nossa escolha é por uma notação mais simples. Por isso, ficará claro, durante o texto, se estamos aplicando ${ }^{\cdot}$ a um elemento tomado de $G$ ou tomado de $M$, de maneira a evitarmos ambiguidade. Finalmente, definimos o grafo bipartido induzido por $\mathbb{K}=(G, M, I)$ como sendo $\mathcal{G}(\mathbb{K})=(U, W, E)$ onde:

$$
\begin{aligned}
& U=\dot{G}, W=\dot{M} \\
& E=\{\{\dot{g}, \dot{m}\} \mid g \in G, m \in M,(g, m) \in I\} .
\end{aligned}
$$

Chamamos de vértices os elementos de $U \cup W$, e os conjuntos $U$ e $W$ de classes de vértices. Os elementos de $E$ são ditos arestas e denotados simplesmente por $\dot{g} \dot{m}$. Quando $\dot{g} \dot{m} \in E$, será dito que $\dot{g}$ e $\dot{m}$ são vértices adjacentes ou vizinhos, e que são as pontas de $\dot{g} \dot{m}$. Daqui em diante, denotaremos $(g, m) \in I$ por $g I m$ e $(g, m) \notin I$ por $g \mp m$.

Em certo sentido, $\mathcal{G}(\mathbb{K})$ carrega a mesma informação da tripla $\mathbb{K}$ : as duas classes de vértices $\dot{G}$ e $\dot{M}$ estão em bijeção, respectivamente, com $G$ e $M$. Além disso, dois vértices $\dot{g} \in \dot{G}$ e $\dot{m} \in \dot{M}$ são adjacentes em $\mathcal{G}(\mathbb{K})$ se e somente se $g I m$. Por exemplo, considere o contexto $\mathbb{K}=(\{1,2,3\},\{1,2,3\}, \neq)$. Uma representação de $\mathbb{K}$ por meio de uma tabela, e uma representação por grafo bipartido são vistos na Figura 2.2.

\begin{tabular}{|c|c|c|c|}
\hline $\mathbb{K}$ & 1 & 2 & 3 \\
\hline 1 & & $\times$ & $\times$ \\
\hline 2 & $\times$ & & $\times$ \\
\hline 3 & $\times$ & $\times$ & \\
\hline
\end{tabular}

(a)

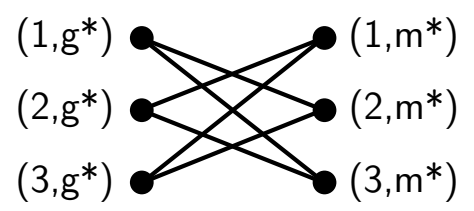

(b)

Figura 2.2: Representações por tabela e grafo bipartido de um contexto. 
A ordem das linhas e das colunas na tabela presente na Figura 2.2(a) é arbitrária e, assim, tabelas obtidas por permutações de suas linhas ou colunas representam o mesmo contexto.

Denotaremos ao longo do texto por $\mathcal{P}(X)$ o conjunto das partes de um conjunto $X$ arbitrário. Mais adiante, faremos um paralelo entre um contexto $\mathbb{K}=(G, M, I)$ e seu grafo $\mathcal{G}(\mathbb{K})$. Preparando a linguagem para isto, mencionamos que subconjuntos de objetos (isto é, subconjuntos $A \subseteq G$ ) serão tratados como subconjuntos de vértices da classe $\dot{G}$. Também serão tratados subconjuntos de atributos como subconjuntos de vértices da classe $\dot{M}$. Para tanto, usaremos bijeções entre $\mathcal{P}(G)$ e $\mathcal{P}(\dot{G})$ e entre $\mathcal{P}(M)$ e $\mathcal{P}(\dot{M})$. A bijeção entre $\mathcal{P}(G)$ e $\mathcal{P}(\dot{G})$ será dada de maneira óbvia, induzida pela identificação dos elementos de $G$ em $\dot{G}$, isto é, a função aplicada em $A \subseteq G$ é definida como

$$
\dot{A}=\{\dot{g} \in \dot{G} \mid g \in A\} .
$$

A bijeção entre $\mathcal{P}(M)$ e $\mathcal{P}(\dot{M})$ será dada de maneira análoga.

\subsection{Operadores de Derivação}

A fim de formalizar as noções de conceito e hierarquia conceitual, define-se em FCA um operador de derivação, que leva um conjunto de objetos $A \subseteq G$ ao conjunto de atributos comuns a todos os objetos $g \in A$. Mais formalmente, definimos a derivação de um conjunto $A \subseteq G$, denotada $A^{\prime}$, por:

$$
A^{\prime}=\{m \in M \mid(\forall g \in A) g I m\} .
$$

Note que a derivação de um conjunto de objetos é uma função ' $: \mathcal{P}(G) \longrightarrow \mathcal{P}(M)$. Veremos a seguir alguns exemplos de derivações tirados do subcontexto dos vertebrados, apresentado na Tabela 2.1.

1. $\{\text { peixe papagaio }\}^{\prime}=\{$ respira na água, possui bico, possui esqueleto, vive na água $\}$.

2. $\{\text { peixe papagaio, peixe lanterna }\}^{\prime}=\{$ respira na água, possui esqueleto, vive na água $\}$.

3. $\{\text { águia, macaco }\}^{\prime}=\{$ possui esqueleto $\}$.

Observe que a derivação de um conjunto unitário $\{g\}$ é simplesmente o conjunto de atributos apresentados por $g$, como pode ser observado no item 1.

Também observe que o conjunto de objetos do item 2 contém propriamente o conjunto de objetos visto no item 1, e o atributo "possui bico" não pertence à sua derivação porque o objeto "peixe lanterna" não possui este atributo. Neste contexto formal, observe que temos que $G^{\prime}=$ \{macaco, peixe papagaio, peixe lanterna, águia $\}^{\prime}=$ \{possui esqueleto\}, ou seja, todo objeto do contexto apresenta o atributo "possui esqueleto" (algo esperado num contexto de animais vertebrados). A derivação de $G$ ser não vazia equivale a dizer que (pelo menos) uma das colunas de $\mathbb{K}$ quando representado por uma tabela é repleta de cruzes $(x)$.

De maneira análoga, também podemos definir um operador de derivação sobre conjuntos de atributos. Mais adiante, veremos que os operadores de derivação que estamos definindo podem ser vistos como intersecção de adjacências em $\mathcal{G}(\mathbb{K})$. 
A derivação de um conjunto $B \subseteq M$, denotada ${ }^{2} B^{\prime}$, é:

$$
B^{\prime}=\{g \in G \mid(\forall m \in B) g I m\} .
$$

A derivação de um conjunto de atributos é uma função ' $: \mathcal{P}(M) \longrightarrow \mathcal{P}(G)$. Tipicamente, nos trabalhos de FCA, denotam-se conjuntos de objetos por $A, A_{1}, A_{2}$, etc. e conjuntos de atributos por $B, B_{1}, B_{2}$, etc.

Retornando ao exemplo da Tabela 2.1, temos por exemplo as seguintes derivações de subconjuntos de atributos:

1. $\{\text { vive na água }\}^{\prime}=\{$ peixe papagaio, peixe lanterna $\}$.

2. $\{\text { possui esqueleto, voa }\}^{\prime}=\{$ águia $\}$.

3. $M^{\prime}=\emptyset$.

4. $\{\text { respira na água, possui esqueleto, vive na água }\}^{\prime}=\{$ peixe papagaio, peixe lanterna $\}$.

Observe que a derivação de um conjunto unitário $\{m\}$ é simplesmente o conjunto de objetos que apresentam o atributo $m$, como pode ser visto no item 1 .

Apesar do atributo "possui esqueleto" ser apresentado por todos os objetos do contexto, apenas "águia" pertence à derivação \{possui esqueleto, voa\}' vista no item 2 pois, "águia" é o único objeto que apresenta o atributo "voa". Como nenhum objeto do contexto apresenta todos os atributos, segue que a derivação de $M$ é vazia. Equivalentemente, nenhuma linha de $\mathbb{K}$, quando visto como tabela, é repleta de cruzes $(\times)$.

\subsubsection{Derivação vista em $\mathcal{G}(\mathbb{K})$}

Considere o grafo bipartido $(\dot{G}, \dot{M}, E)$ associado a um contexto qualquer. Sejam $\dot{g} \in \dot{G}$ e $\dot{m} \in \dot{M}$. Definimos a vizinhança de $\dot{g}, N(\dot{g})$, como sendo o conjunto de vértices em $\dot{M}$ que são adjacentes a $\dot{g}$ em $\mathcal{G}$, e analogamente para $\dot{m}$, isto é:

$$
N(\dot{g})=\{\dot{m} \in \dot{M} \mid \dot{g} \dot{m} \in E\} \quad \text { e } \quad N(\dot{m})=\{\dot{g} \in \dot{G} \mid \dot{g} \dot{m} \in E\} .
$$

No que seguirá, tomaremos conjuntos $\dot{A} \subseteq \dot{G}$ e $\dot{B} \subseteq \dot{M}$ e consideraremos a intersecção de vizinhanças $\cap_{\dot{g} \in \dot{A}} N(\dot{g})$ e $\cap_{\dot{m} \in \dot{B}} N(\dot{m})$. Assim, convencionamos que, se $\dot{A}$ for vazio, então a intersecção das vizinhanças de seus elementos vale $\dot{M}$, e, da mesma maneira, $\cap_{\dot{m} \in \dot{B}} N(\dot{m})$ vale $\dot{G}$ quando $\dot{B}$ for vazio.

O operador de derivação pode ser entendido de uma forma intuitiva ao nos depararmos com o grafo associado a um contexto. A construção de $\mathcal{G}$ foi realizada de maneira que a seguinte equivalência vale:

O objeto $g$ possui o atributo $m \Leftrightarrow g I m$

$$
\begin{aligned}
& \Leftrightarrow \text { os vértices } \dot{g} \in \dot{G} \text { e } \dot{m} \in \dot{M} \text { são adjacentes em } \mathcal{G}(\mathbb{K}) \\
& \Leftrightarrow \dot{g} \dot{m} \in E .
\end{aligned}
$$

\footnotetext{
${ }^{2}$ A utilização do mesmo símbolo de derivação para conjuntos de atributos e de objetos é um abuso de linguagem que simplifica a notação, mas exige cuidado para evitar ambiguidade. Se $X$ for um conjunto, deve estar claro se estamos tomando $X \subseteq G$ ou $X \subseteq M$, para não haver ambiguidade quanto ao conjunto $X^{\prime}$.
} 
Então, seja $A \subseteq G$ e fixemos $m \in M$. Assim, temos que:

$$
\begin{aligned}
m \in A^{\prime} \Leftrightarrow(\forall g \in A) g I m & \Leftrightarrow(\forall \dot{g} \in \dot{A}) \dot{g} \dot{m} \in E \\
& \Leftrightarrow \dot{m} \in \bigcap_{\dot{g} \in \dot{A}} N(\dot{g}) .
\end{aligned}
$$

Ou seja, em $\mathcal{G}$, o conjunto $A^{\prime}$ corresponde aos vértices de $\dot{M}$ que são adjacentes a cada $\dot{g} \in \dot{A}$.

Os itens (a) e (b) da Figura 2.3 ilustram alguns exemplos de derivações de objetos num subcontexto dos vertebrados. Vértices azuis da classe $\dot{G}$ são vértices associados aos objetos de $A$ e os vértices azuis da classe $\dot{M}$ são vértices associados aos atributos pertencentes a $A^{\prime}$. Uma aresta é azul se e só se ambas suas pontas são vértices azuis. A situação análoga para conjuntos de atributos está representada nos itens (c) e (d) da mesma figura.

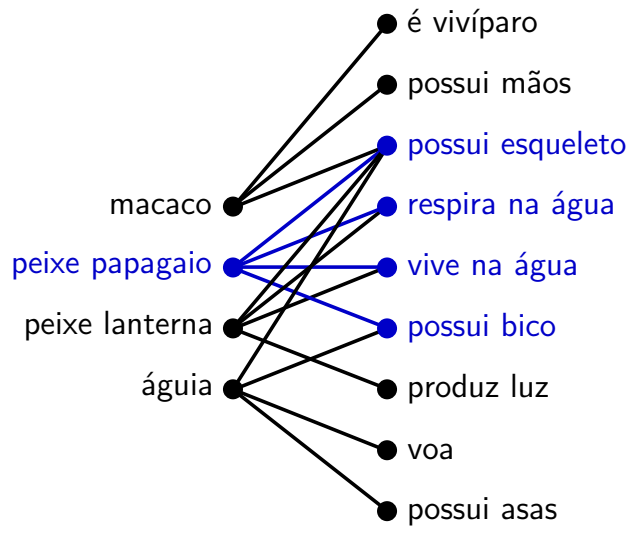

(a) Derivação de \{peixe papagaio\}

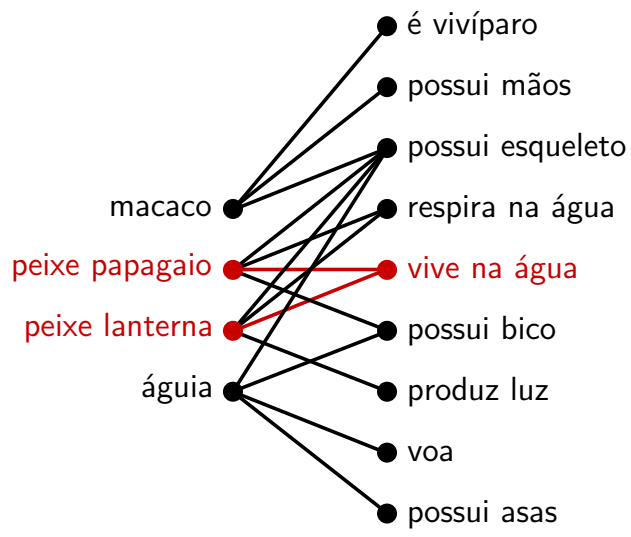

(c) Derivação de \{vive na água\}

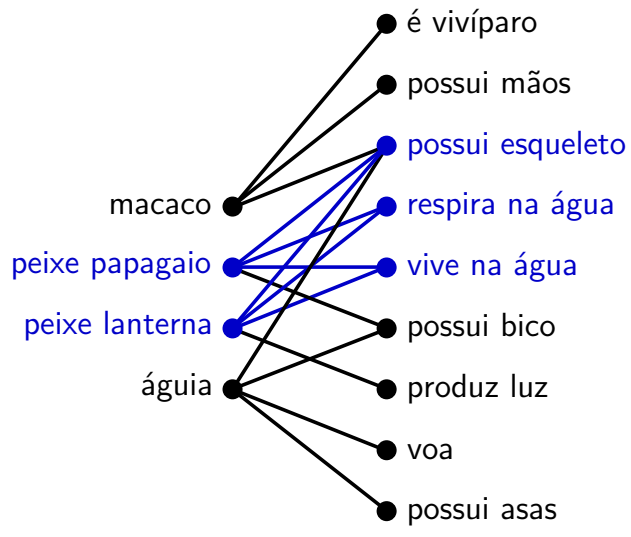

(b) Derivação de \{peixe papagaio, peixe lanterna\}

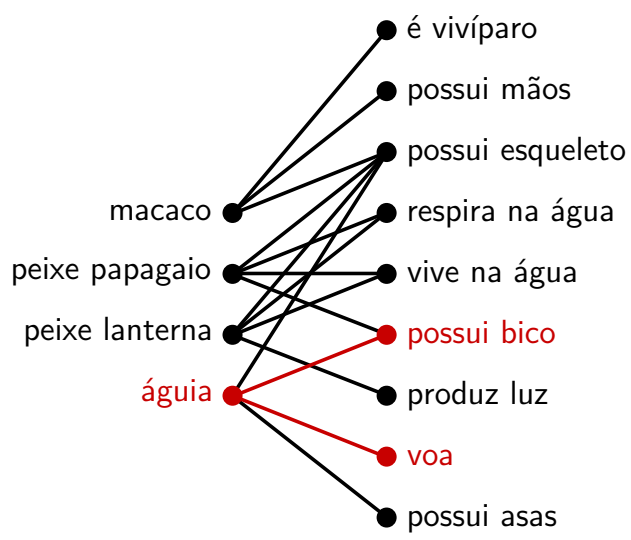

(d) Derivação de \{possui bico, voa\}

Figura 2.3: Derivações de conjuntos de objetos e de atributos, quando vistas em $\mathcal{G}(\mathbb{K})$. 


\subsubsection{Bicliques de $\mathcal{G}(\mathbb{K})$}

Após termos mostrado como se reflete a derivação de um conjunto de objetos $A$ (e de atributos $B$ ) no grafo $\mathcal{G}$, estamos interessados em considerar $A$ e sua derivação, $A^{\prime}$, como conjuntos de vértices de $\mathcal{G}$ e investigar se estes correspondem a algum objeto matemático notável em $\mathcal{G}$. Veremos que o objeto em questão é o que chamaremos de biclique, e que este possui uma propriedade de maximalidade.

Dado um grafo bipartido $(U, W, E)$, uma biclique é um par $(A, B)$ com $A \subseteq U, B \subseteq W$ tal que $u w \in E$ para todo $u \in A$ e todo $w \in B$. Se $(A, B)$ for uma biclique e não existir biclique $(A, D)$ com $D \supsetneq B$, então chamamos esta biclique de $W$-maximal. Analogamente, se não existir biclique $(C, B) \operatorname{com} C \supsetneq A$, então a chamamos de biclique $U$-maximal. Se uma biclique for $U$-maximal e $W$-maximal, então a chamamos de biclique maximal.

Considere um conjunto de objetos $A \subseteq G$, defina $B=A^{\prime}$ e considere o par $(\dot{A}, \dot{B})$ de subconjuntos de vértices de $\mathcal{G}$. Claramente tal par é uma biclique, uma vez que $B=A^{\prime}$ e portanto, para todo $g \in A$ e todo $m \in B$, temos que $g I m$, o que equivale a $\dot{g} \dot{m} \in E(\mathcal{G})$. A próxima proposição mostra que as bicliques desta forma são $\dot{M}$-maximais. Naturalmente, ao considerarmos $B \subseteq M$ e definirmos $A=B^{\prime}$, teremos um resultado análogo.

Proposição 2.1. Sejam $(G, M, I)$ um contexto, $\mathcal{G}=(\dot{G}, \dot{M}, E)$ seu grafo bipartido associado e sejam $A \subseteq G$ e $B \subseteq M$. Então:

i) $S e B=A^{\prime}$, então, para todo $D \subseteq M$ tal que $(\dot{A}, \dot{D})$ é uma biclique, vale que $D \subseteq B$. Em particular, $(\dot{A}, \dot{B})$ é uma biclique $\dot{M}$-maximal.

ii) Se $A=B^{\prime}$, então, para todo $C \subseteq G$ tal que $(\dot{C}, \dot{B})$ é uma biclique, vale que $C \subseteq A$. Em particular, $(\dot{A}, \dot{B})$ é uma biclique $\dot{G}$-maximal.

Prova. Suponha o caso em que $B=A^{\prime}$. Seja $(\dot{A}, \dot{D})$ uma biclique de $\mathcal{G}$. Pela definição de biclique, temos que

$$
\begin{aligned}
(\forall \dot{g} \in \dot{A})(\forall \dot{m} \in \dot{D}) \dot{g} \dot{m} \in E & \Leftrightarrow(\forall g \in A)(\forall m \in D) g I m \\
& \Leftrightarrow(\forall m \in D) m \in A^{\prime} \\
& \Leftrightarrow D \subseteq A^{\prime}=B .
\end{aligned}
$$

Assim, em particular, não vale que $D$ contém propriamente $B$. Logo, segue que não existe biclique $(\dot{A}, \dot{D})$ com $\dot{D} \supsetneq \dot{B}$, isto é, a biclique $(\dot{A}, \dot{B})$ é $\dot{M}$-maximal. $\mathrm{O}$ caso ii) segue analogamente.

Uma consequência da Proposição 2.1 é que pares $(A, B)$ satisfazendo $A=B^{\prime}$ e $B=A^{\prime}$ correspondem a bicliques maximais em $\mathcal{G}$. Tais pares serão alvo da próxima definição, a ser feita na seção seguinte.

\subsection{Conceitos formais}

Dado um contexto $(G, M, I)$, um conceito formal (ou simplesmente conceito) é um par $(A, B)$, com $A \subseteq G, B \subseteq M$, satisfazendo $A^{\prime}=B$ e $B^{\prime}=A$. Além disso, chamamos $A$ de extensão e $B$ de intenção do conceito $(A, B)$. 
Tomemos, por exemplo, $A=\{$ peixe papagaio, peixe lanterna $\}$ e $B=\{$ respira na água, possui esqueleto, vive na água\}. No item 2 dos exemplos de derivações de objetos verificamos que $A^{\prime}=B$. Também foi verificado, no item 4 dos exemplos de derivações de atributos, que $B^{\prime}=A$. Assim, o par $(A, B)$ é um conceito.

Vimos na Proposição 2.1 que conceitos de um contexto correspondem a bicliques maximais do seu grafo associado. É fácil mostrar que também há uma correspondência no sentido contrário, e isto será feito na próxima proposição.

A Proposição 2.2 também mostrará que extensões podem ser caracterizadas como conjuntos $A \subseteq G$ fixados pelo operador de derivação dupla, isto é, $A=A^{\prime \prime}$, e, analogamente, intenções são subconjuntos $B \subseteq M$ com $B^{\prime \prime}=B$. Definimos que um subconjunto de objetos (atributos) fixados pelo respectivo operador de derivação dupla é um fechado. A razão desta denominação, fechado, ficará clara após uma observação que será feita em seguida da demonstração da Proposição 2.3.

Proposição 2.2. Seja $\mathbb{K}=(G, M, I)$ um contexto, $\mathcal{G}$ o seu grafo bipartido associado $e$ sejam $A \subseteq G$ e $B \subseteq M$. São equivalentes:

i) $(A, B)$ é um conceito de $\mathbb{K}$;

ii) $A$ derivação de $A$ é $B$ e $A$ é fechado;

iii) $A$ derivação de $B$ é $A$ e $B$ é fechado;

iv) $(\dot{A}, \dot{B})$ é uma biclique maximal de $\mathcal{G}$.

Prova. i) $\Rightarrow$ iv): Suponha que $(A, B)$ seja um conceito. Logo, $A=B^{\prime}$ e $B=A^{\prime}$ e portanto, pela Proposição 2.1, temos que $(\dot{A}, \dot{B})$ é uma biclique $\dot{G}$-maximal e $\dot{M}$-maximal.

iv) $\Rightarrow$ i): Seja $(\dot{A}, \dot{B})$ uma biclique maximal de $\mathcal{G}$. Provaremos que $A^{\prime}=B$, e a demonstração de que $B=A^{\prime}$ é análoga.

Do fato de $(\dot{A}, \dot{B})$ ser biclique, temos que $\dot{g} \dot{m} \in E$ para todo $\dot{g} \in \dot{A}$ e todo $\dot{m} \in \dot{B}$, e portanto,

$$
(\forall g \in A)(\forall m \in B) g I m \Leftrightarrow(\forall m \in B) m \in A^{\prime},
$$

e então $B \subseteq A^{\prime}$. Por hipótese, em particular, a biclique $(\dot{A}, \dot{B})$ é $\dot{M}$-maximal. Assim, para todo $\dot{m} \in \dot{M} \backslash \dot{B}$, existe $\dot{g} \in A$ tal que $\dot{g} \dot{m} \notin E$. Logo,

$$
(\forall m \in M \backslash B)(\exists g \in A) g \mp m
$$

de onde segue que $m \notin A^{\prime}$, para todo $m \in M \backslash B$. Logo, $A^{\prime} \subseteq B$ e, portanto, $B=A^{\prime}$.

i) $\Rightarrow$ ii): Seja $(A, B)$ um conceito. Da definição de conceito, segue de imediato que $A^{\prime}=B$ e portanto $A^{\prime \prime}=B^{\prime}=A$.

ii) $\Rightarrow$ i): Suponha que $A^{\prime}=B$ e $A^{\prime \prime}=A$. Substituindo, temos que $B^{\prime}=A^{\prime \prime}=A$, e assim, segue que $A^{\prime}=B$ e $B^{\prime}=A$.

Mostramos a equivalência entre i), ii) e iv). A equivalência entre i) e iii) pode ser mostrada de maneira análoga à demonstração de que i) e ii) são equivalentes. 
Na Figura 2.4(a) está representado um conceito, que pode ser entendido como o conceito de peixes do subcontexto dos vertebrados, enquanto que na Figura 2.4(b), vemos o que pode ser entendido como o conceito de vertebrados. Denotaremos o conjunto de conceitos de um contexto $\mathbb{K}=(G, M, I)$ por $\mathfrak{B}(\mathbb{K})$ ou por $\mathfrak{B}(G, M, I)$.

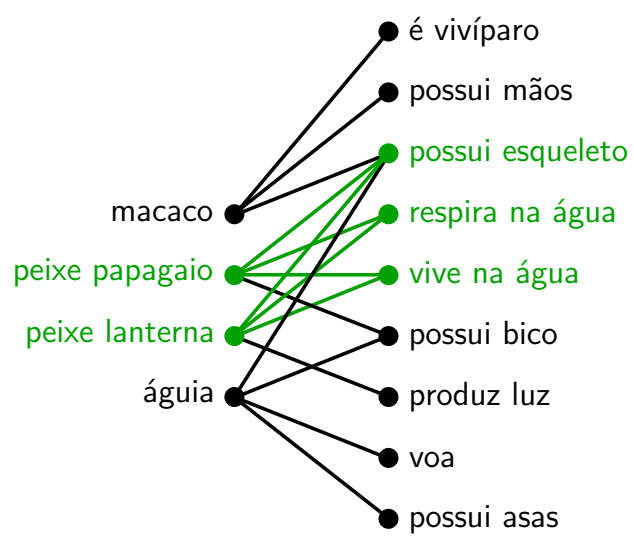

(a) Conceito dos peixes

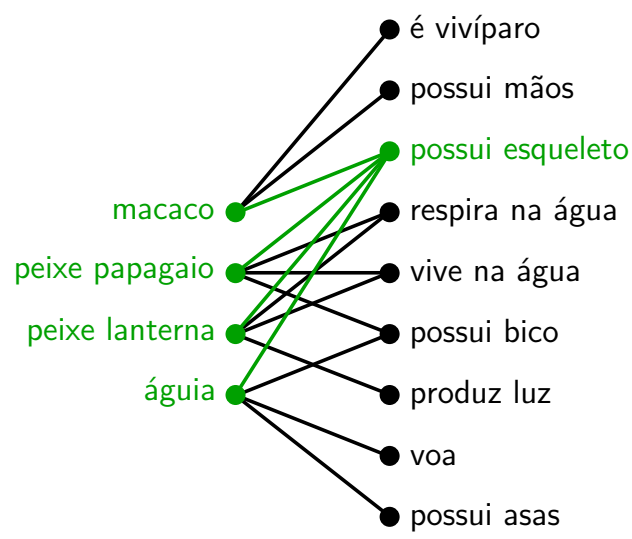

(b) Conceito dos vertebrados

Figura 2.4: Duas bicliques maximais em $\mathcal{G}(\mathbb{K})$ representando conceitos.

O conteúdo da Proposição 2.2 é conhecido há algum tempo na comunidade de FCA. Como nos foi comunicado [Gan10], uma das primeiras referências é [Sch82]. No entanto, não temos acesso a esse trabalho, tampouco a quaisquer referências que apresentem este conteúdo. Assim, o que foi apresentado aqui é uma formalização própria. O valor do paralelo realizado entre bicliques maximais e conceitos é a possibilidade de se utilizar resultados da teoria dos grafos em FCA. Esta utilização será feita ao longo do Capítulo 7.

\subsection{Hierarquias de conceitos}

O objetivo buscado nesta seção é o de definir a noção de hierarquia de conceitos. Isso se dará através da definição de uma ordem parcial sobre $\mathfrak{B}(G, M, I)$. Havendo uma ordem parcial sobre conceitos, poderemos expressar, por exemplo, que o conceito de peixes, visto na Figura 2.4(a), é "mais específico" que o conceito de vertebrados, visto na Figura 2.4(b). Equivalentemente, diremos que o conceito de vertebrados é "mais geral" que o conceito de peixes. Mas, antes de definirmos formalmente esta relação entre conceitos, precisamos provar propriedades básicas dos operadores de derivação que nos serão muito úteis.

Proposição 2.3. Seja $\mathbb{K}=(G, M, I)$ um contexto. Os operadores de derivação satisfazem as seguintes propriedades:

(i) $A_{1} \subseteq A_{2} \Rightarrow A_{1}^{\prime} \supseteq A_{2}^{\prime}$

(ii) $A \subseteq A^{\prime \prime}$

(iii) $A^{\prime}=A^{\prime \prime \prime}$

(iv) $A \subseteq B^{\prime} \Leftrightarrow A^{\prime} \supseteq B$, (i') $B_{1} \subseteq B_{2} \Rightarrow B_{1}^{\prime} \supseteq B_{2}^{\prime}$

(ii') $B \subseteq B^{\prime \prime}$

(iii') $B^{\prime}=B^{\prime \prime \prime}$

para quaisquer $A, A_{1}, A_{2} \subseteq G$ e quaisquer $B, B_{1}, B_{2} \subseteq M$. 
Prova. Serão provados (i), (ii), (iii) e (iv). Da mesma maneira, seguem (i'), (ii') e (iii').

(i) Seja $m \in A_{2}^{\prime}$. Então temos que $g I m$, para todo $g \in A_{2}$. Como $A_{2} \supseteq A_{1}$, temos em particular, que $g I m$ para todo $g \in A_{1}$. Logo, $m \in A_{1}^{\prime}$, e portanto $A_{2}^{\prime} \subseteq A_{1}^{\prime}$.

(ii) Seja $g \in A$. Considere $A^{\prime}=\{m \in M \mid(\forall g \in A) g I m\}$. Em particular, para cada $m \in A^{\prime}$, temos que $g I m$. Logo, $g \in A^{\prime \prime}=\left\{g \in G \mid\left(\forall m \in A^{\prime}\right) g I m\right\}$ e, assim, $A \subseteq A^{\prime \prime}$.

(iii) Para provar $A^{\prime} \subseteq A^{\prime \prime \prime}$, basta tomar $B=A^{\prime}$ no item (ii'). Pelo item (ii), temos que $A \subseteq A^{\prime \prime}$. Aplicando o item (i) com $A_{1}=A$ e $A_{2}=A^{\prime \prime}$, temos que $A^{\prime} \supseteq A^{\prime \prime \prime}$.

(iv) Suponha que $A \subseteq B^{\prime}$. Por (i), segue que $A^{\prime} \supseteq B^{\prime \prime}$. Do item (ii'), vale que $B^{\prime \prime} \supseteq B$. Por transitividade, temos que $A^{\prime} \supseteq B$. Agora, suponha o caso $A^{\prime} \supseteq B$. Pelo item (i'), segue que $A^{\prime \prime} \subseteq B^{\prime}$. Do item (ii), vale que $A \subseteq A^{\prime \prime}$. Por transitividade, temos que $A \subseteq B^{\prime}$

É possível aplicar, de maneira elementar, as propriedades mostradas na Proposição 2.3 para concluir que os operadores de derivação dupla: " $: \mathcal{P}(G) \rightarrow \mathcal{P}(G) \mathrm{e}^{\prime \prime}: \mathcal{P}(M) \rightarrow \mathcal{P}(M)$ satisfazem as seguintes propriedades:

- extensividade: $A \subseteq A^{\prime \prime}$ e $B \subseteq B^{\prime \prime}$;

- idempotência: $A^{\prime \prime \prime \prime}=A^{\prime \prime}$ e $B^{\prime \prime \prime \prime}=B^{\prime \prime}$;

- monotonicidade crescente: $A_{1} \subseteq A_{2} \Rightarrow A_{1}^{\prime \prime} \subseteq A_{2}^{\prime \prime}$ e $B_{1} \subseteq B_{2} \Rightarrow B_{1}^{\prime \prime} \subseteq B_{2}^{\prime \prime}$;

para quaisquer $A \subseteq G$ e $B \subseteq M$. Qualquer função satisfazendo as três propriedades acima é dita um operador de fecho. Como foi adiantado antes da Proposição 2.2, conjuntos iguais aos seus respectivos fechos são chamados de fechados. Utilizando parte dos conteúdos das Proposições 2.2 e 2.3, e ainda fazendo uso destas últimas definições, podemos resumir: operadores de derivação dupla são sempre operadores de fecho e, seus fechados são extensões ou intenções.

Outra consequência da proposição anterior é que os conceitos de um contexto $\mathbb{K}$ podem ser naturalmente submetidos a uma ordem parcial, da seguinte maneira:

$$
\left(A_{1}, B_{1}\right) \leq\left(A_{2}, B_{2}\right) \Leftrightarrow A_{1} \subseteq A_{2} .
$$

Neste caso, dizemos que o conceito $\left(A_{1}, B_{1}\right)$ é mais específico que $\left(A_{2}, B_{2}\right)$, ou ainda, o conceito $\left(A_{2}, B_{2}\right)$ é mais geral que $\left(A_{1}, B_{1}\right)$. É trivial demonstrar que esta relação é uma ordem parcial sobre $\mathfrak{B}(G, M, I)$.

A assimetria da definição (2.1) é meramente ilusória: para dois conceitos $\left(A_{1}, B_{1}\right)$ e $\left(A_{2}, B_{2}\right)$, tem-se que $A_{1} \subseteq A_{2}$ se e somente se $B_{1} \supseteq B_{2}$ pois, por definição, $B_{1}=A_{1}^{\prime} \mathrm{e}$ $B_{2}=A_{2}^{\prime}$, e ainda o item (i) da Proposição 2.3 garante que $A_{1} \subseteq A_{2} \Rightarrow B_{1}=A_{1}^{\prime} \supseteq A_{2}^{\prime}=B_{2}$. Reciprocamente, se $B_{1} \supseteq B_{2}$, então o item (i') da mesma proposição garante que $B_{1}^{\prime} \subseteq B_{2}^{\prime}$ e, portanto, que $A_{1} \subseteq A_{2}$. Assim:

$$
\left(A_{1}, B_{1}\right) \leq\left(A_{2}, B_{2}\right) \Leftrightarrow A_{1} \subseteq A_{2} \Leftrightarrow B_{1} \supseteq B_{2} .
$$


Veremos adiante que a ordem parcial definida em (2.1) apresenta propriedades especiais, mais especificamente, a propriedade de ser um reticulado. Este fato justifica o nome reticulado de conceitos para a estrutura $\underline{\mathfrak{B}}(G, M, I)=\langle\mathfrak{B}(G, M, I), \leq\rangle$, onde $\leq$ é como definido em (2.1). Como não estabelecemos ainda que a estrutura $\underline{\mathfrak{B}}(G, M, I)$ é, de fato, um reticulado, chamála-emos por ora de hierarquia de conceitos de $\mathbb{K}$.

Como exemplo, segue representada na Figura 2.5 a hierarquia de conceitos do subcontexto dos vertebrados, através de um diagrama de Hasse, a ser descrito na Seção 2.5.

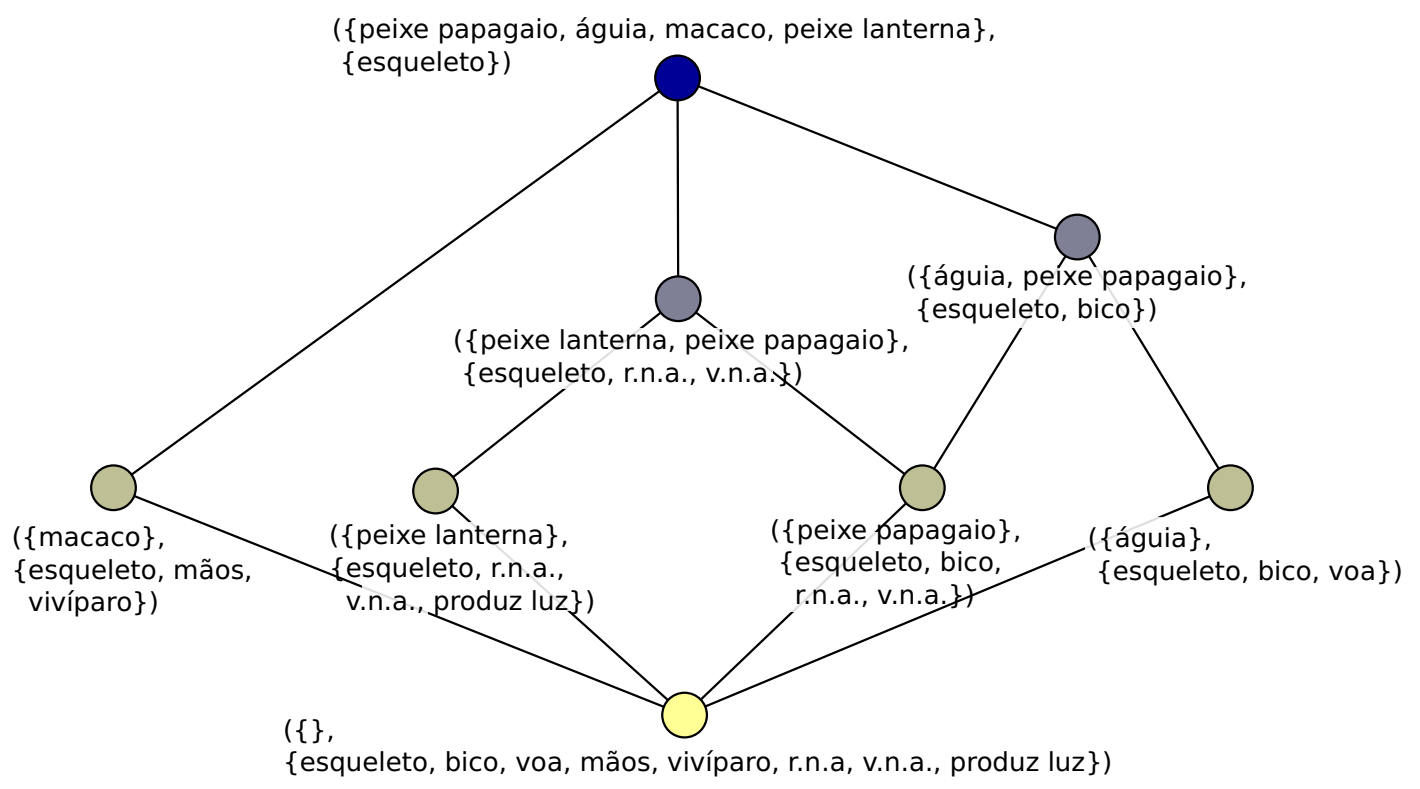

Figura 2.5: Hierarquia de conceitos com rotulação cheia.

No diagrama da Figura 2.5, cada conceito está rotulado com sua extensão e intenção. Esta é a chamada rotulação cheia. Para facilitar a apresentação destes rótulos, muitos atributos foram abreviados. Em particular, o atributo "vive na água" foi abreviado para "v.n.a.", "respira na água" para "r.n.a.", e assim semelhantemente com outros atributos. Esta rotulação apresenta redundância de informação e geralmente torna poluídos os diagramas de Hasse de hierarquias de conceitos.

A redundância de uma rotulação cheia pode ser notada observando-se que, para todo conceito $(A, B)$, cada elemento $g \in A$ pertence a todas as extensões dos conceitos mais gerais que ele. Semelhantemente, para todo conceito $(A, B)$, cada elemento $m \in B$ pertence a todas as intenções dos conceitos mais específicos que $(A, B)$.

As seguintes funções $\gamma: G \rightarrow \underline{\mathfrak{B}}(G, M, I)$ e $\mu: M \rightarrow \underline{\mathfrak{B}}(G, M, I)$ levam, respectivamente, um objeto ao conceito mais específico que contém $g$ como objeto e um atributo $m$ ao conceito mais geral que contém $m$ como atributo:

$$
\begin{aligned}
\gamma: G & \rightarrow \underline{\mathfrak{B}}(G, M, I) & \mu: M & \rightarrow \underline{\mathfrak{B}}(G, M, I) \\
g & \mapsto\left(\{g\}^{\prime \prime},\{g\}^{\prime}\right) & m & \mapsto\left(\{m\}^{\prime},\{m\}^{\prime \prime}\right) .
\end{aligned}
$$

A seguinte maneira de rotular uma hierarquia $\underline{\mathfrak{B}}(G, M, I)$ é chamada de rotulação reduzida: 
- Para todo $g \in G$, o conceito $\gamma(g)$ recebe o rótulo $g$;

- Para todo $m \in M$, o conceito $\mu(m)$ recebe o rótulo $m$.

As funções $\gamma$ e $\mu$ não são, em geral, injetoras. O rotulamento acima é cumulativo, no sentido de que, se $(A, B)=\gamma\left(g_{i}\right)=\gamma\left(g_{j}\right)$, então o conceito $(A, B)$ recebe ambos os objetos $g_{i}$ e $g_{j}$ como rótulos. O mesmo vale para casos onde $(A, B)=\mu\left(m_{i}\right)=\mu\left(m_{j}\right)$, recebendo o conceito $(A, B)$ rótulos de ambos os atributos $m_{i}$ e $m_{j}$. Por convenção, ao realizar uma rotulação reduzida, rótulos de atributos são posicionados em cima de conceitos, enquanto rótulos de objetos são posicionados embaixo de conceitos. O diagrama de Hasse da Figura 2.6 foi rotulado de maneira reduzida, e podemos observar que $\mu$ não é injetora, pois aplica "respira na água" e "vive na água" em um mesmo conceito.

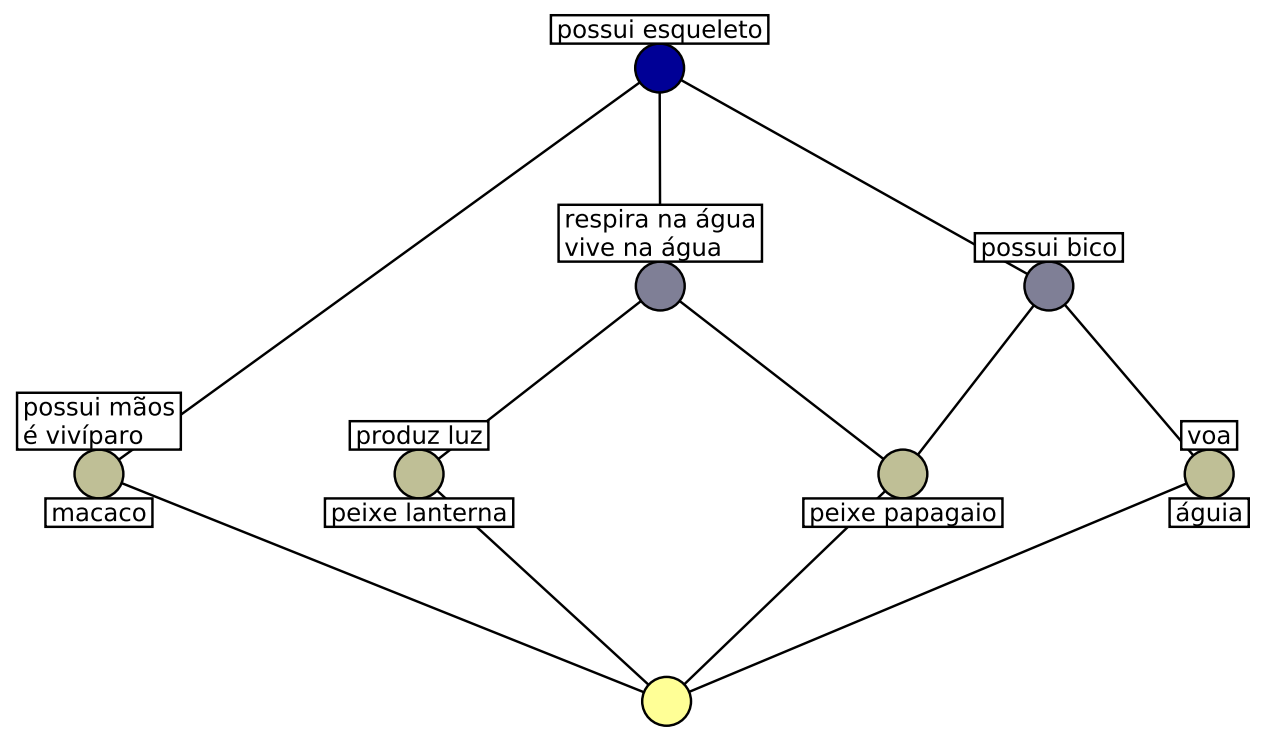

Figura 2.6: Hierarquia de conceitos com rotulação reduzida.

É possível recuperar a rotulação cheia a partir da rotulação reduzida. A extensão de um conceito $(A, B)$, isto é, $A$, é dada pela união dos rótulos atribuidos a conceitos mais específicos ou iguais a $(A, B)$. Como exemplo, seja $(A, B)$ o conceito que recebeu os rótulos "respira na água" e "vive na água" na Figura 2.7. O conjunto $A$ é dado pela união dos rótulos posicionados abaixo dos conceitos presentes no interior da elipse representada naquela figura. Ou seja, $A=\{$ peixe lanterna, peixe papagaio $\}$.

Dualmente, a intenção $B$ daquele conceito é dado pela união dos rótulos posicionados acima de conceitos mais gerais que $(A, B)$. Assim, é possível observar pela Figura 2.8 que $B$ é o conjunto \{respira na água, vive na água, possui esqueleto\}.

Em ambos os tipos de rotulamento (cheio ou reduzido), é comum que os conceitos recebam cores associadas ao tamanho de sua extensão. Mais especificamente, no nosso caso, um conceito $(A, B)$ recebe uma cor que varia do amarelo até o azul ${ }^{3}$, em função do quanto vale a razão $|A| /|G|$, onde $G$ é o conjunto de objetos do contexto.

\footnotetext{
${ }^{3}$ Em impressão monocromática, uma cor que varia do branco até o preto, passando por tons de cinza.
} 


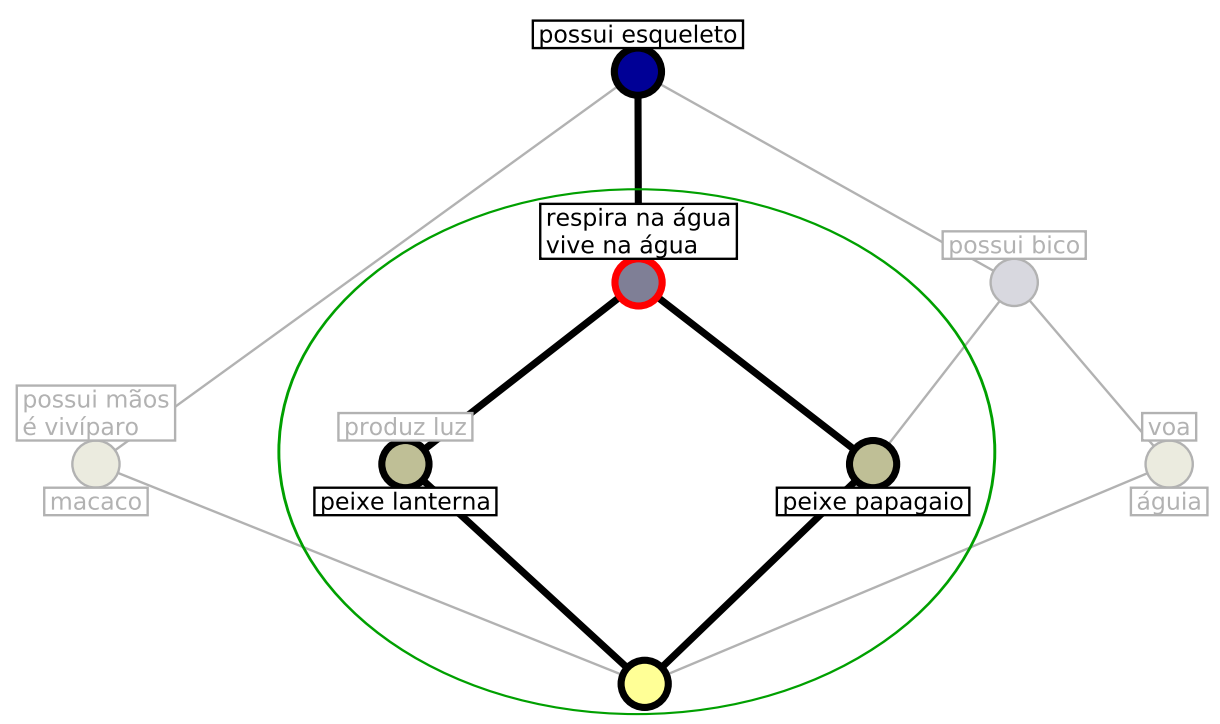

Figura 2.7: Recuperar extensão: toma-se rótulos abaixo de conceitos no interior da elipse.

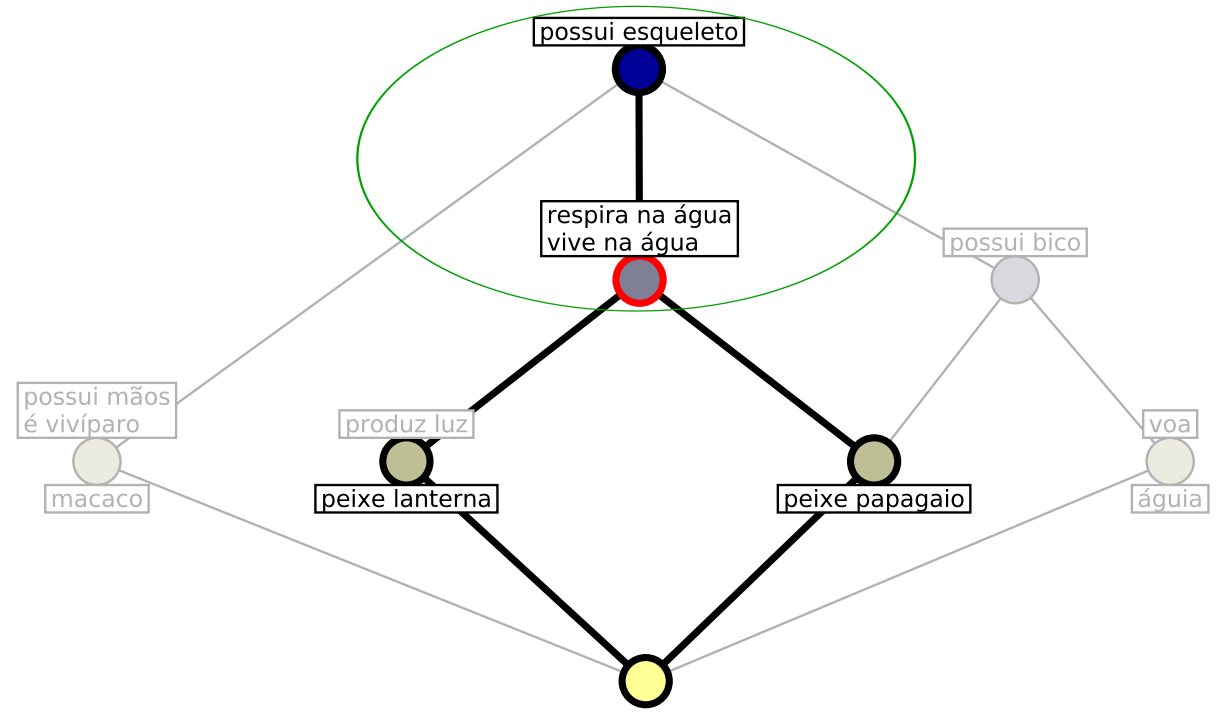

Figura 2.8: Recuperar intenção: toma-se rótulos acima de conceitos no interior da elipse.

Será mostrada no Capítulo 4, sob o foco de aplicações computacionais, a utilização de representações gráficas de reticulados de conceitos. Naquelas aplicações, o tipo de rotulamento desempenha papel central em tarefas de análise de dados.

\subsection{Um pouco de teoria de ordem e de reticulados}

Antes de mostrarmos a caracterização das hierarquias de conceitos na Seção 2.7, faremos uma rápida exposição de elementos básicos da teoria de ordem e de reticulados que serão necessários. 


\subsubsection{Definições}

Um conjunto parcialmente ordenado ou uma ordem parcial é um par $\langle P, \leq\rangle$ onde $\leq$ é uma relação binária sobre $P$ satisfazendo:

1) Reflexividade: $x \leq x$, para todo $x$ em $P$.

1) Anti-simetria: se $x \leq y$ e $y \leq x$, então $x=y$, para todo $x, y \in P$.

3) Transitividade: se $x \leq y$ e $y \leq z$, então $x \leq z$, para todo $x, y, z \in P$.

Toda relação satisfazendo estes três axiomas induz uma relação irreflexiva e transitiva, chamada de ordem estrita, denotada por $<$ e definida da seguinte maneira: $x<y$ se e só se $x \leq y$ e $x \neq y$.

Quando estiver claro qual é a ordem $\leq$ em questão, cometemos o abuso de dizer somente que $P$ é uma ordem parcial. Será dito que $y$ cobre $x$ ou $x$ é coberto por $y$, e denotaremos isso por $x \prec y$, se e só se $x<y$ e, para todo $z \in P$, se $x \leq z<y$ então $z=x$.

Se $P$ for finito, podemos representar a estrutura $\langle P, \leq\rangle$ através de um diagrama de Hasse. Tal diagrama é um grafo orientado, $\mathcal{D}=(V, A)$, onde $V=P$ e existe um arco $(x, y) \in A$ se e somente se $x, y \in P$ e $x \prec y$. Por convenção, toda representação de $\mathcal{D}$ no plano é feita de maneira que, se houver arco $(x, y) \in A$, então o vértice $x$ é representado em um ponto do plano com $Y$-coordenada menor do que $y$. Esta convenção permite omitir a orientação dos arcos de $\mathcal{D}$ quando o representamos no plano. Vemos alguns exemplos na Figura 2.9.

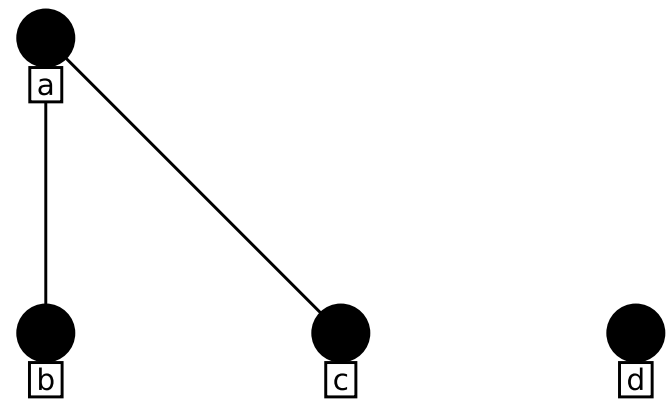

(a) Uma ordem parcial onde $b<a$ e $c<a$.

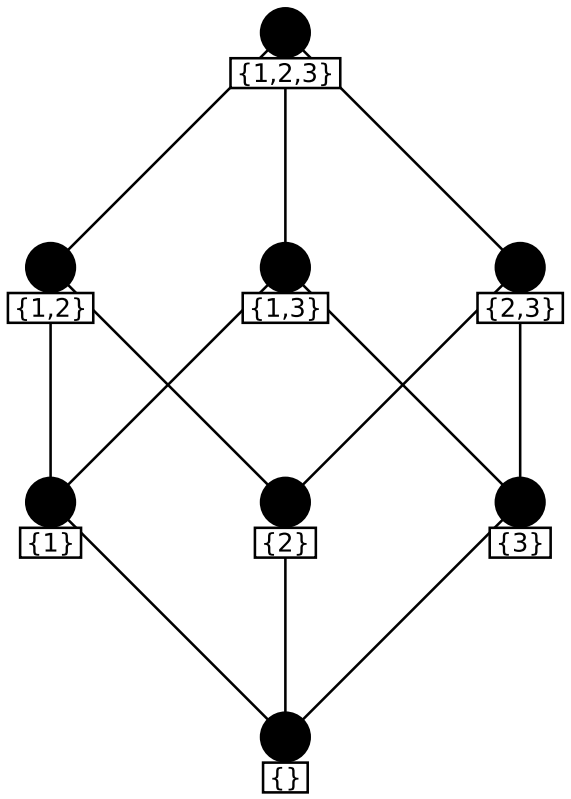

(b) $\langle\mathcal{P}(\{1,2,3\}), \subseteq\rangle$.

Figura 2.9: Diagramas de Hasse.

Um conjunto parcialmente ordenado $\langle P, \leq\rangle$ possui mínimo se e somente se existe $\perp \in P$ tal que $\perp \leq x$ para todo $x \in P$. Neste caso, $\perp$ é chamado de elemento mínimo de $P$. 
Analogamente, $(P, \leq)$ possui máximo se e somente se existir $\top \in P$ tal que $x \leq \top$ para todo $x \in P$. Neste caso, $\top$ é chamado de elemento máximo de $P$. Elementos que cobrem $\perp$ são chamados átomos e elementos que são cobertos por $T$ são chamados coátomos.

Todo subconjunto $S$ de um conjunto parcialmente ordenado $P$ torna-se um conjunto parcialmente ordenado ao restringirmos a relação $\leq$ de $P$ para pares de elementos de $S$. Dado $S \subseteq P$, o conjunto dos limitantes superiores de $S$ em $P$ é denotado e definido da seguinte maneira:

$$
S^{u}=\{x \in P \mid(\forall s \in S) s \leq x\}
$$

Analogamente, o conjunto dos limitantes inferiores de $S$ em $P$ é:

$$
S^{l}=\{x \in P \mid(\forall s \in S) x \leq s\}
$$

Se $S$ for subconjunto de $P$ e $S^{u}$ possuir mínimo, denotamos o (único) elemento mínimo de $S^{u}$ por sup $S$ e o chamamos de supremo de $S$. Também, se $S^{l}$ possuir máximo, chamamos o elemento máximo de $S^{l}$ de ínfimo de $S$ e o denotamos por inf $S$.

Um reticulado é um conjunto parcialmente ordenado $P$ tal que existem $\inf \{x, y\}$ e $\sup \{x, y\}$ para todo $x, y \in P$. Neste caso, denotamos, respectivamente, por $x \wedge y$ e $x \vee y$ o ínfimo e o supremo mencionados. É claro que estas definições de $\wedge$ e $\vee$ resultam em duas operações binárias sobre $P$.

\subsubsection{Exemplos}

Uma cadeia ou ordem total é um conjunto ordenado $P$, tal que, para todo $x, y \in P$, tem-se que $x \leq y$ ou $y \leq x$. Toda cadeia é um reticulado, $\operatorname{com} x \vee y=\max \{x, y\}$, assim como $x \wedge y=\min \{x, y\}$. Dado um conjunto $X$, o conjunto das partes de $X, \mathcal{P}(X)$, torna-se um reticulado após ser equipado com a relação $\subseteq$. Chamaremos este reticulado de reticulado booleano. Nele, os supremos são dados por $A_{1} \vee A_{2}=A_{1} \cup A_{2}$ e $A_{1} \wedge A_{2}=A_{1} \cap A_{2}$, para quaisquer $A_{1}, A_{2} \in \mathcal{P}(X)$.

Considere $\mathbb{N}=\{0,1,2,3, \ldots\}$. A estrutura $\langle\mathbb{N}, \unrhd\rangle$, onde $n \unrhd m$ se e só se $n$ divide $m$ é um reticulado, com $n \vee m=m m c(n, m)$ e $n \wedge m=m d c(n, m)$.

Não é possível representar efetivamente através de uma figura o diagrama de Hasse da cadeia $\langle\mathbb{N}, \leq\rangle$, mas podemos sugerir sua estrutura da maneira mostrada na Figura 2.10(b). Usaremos a notação $[n]$ para nos referirmos ao subconjunto de naturais $\{1,2, \ldots, n\}$. O subconjunto $[7]=\{1,2, \ldots, 7\}$ de $\mathbb{N}$, quando equipado com a ordem $\unrhd$, torna-se um conjunto parcialmente ordenado, mas não um reticulado. No entanto, um diagrama de [7] pode auxiliar a analisar a estrutura de $\langle\mathbb{N}, \unrhd\rangle$. Faremos algumas afirmações sobre $\langle\mathbb{N}, \unrhd\rangle$ que podem ser sugeridas pelo diagrama da Figura 2.10(a), e por fim, demonstradas. O elemento mínimo é 1, e o conjunto de átomos é exatamente o conjunto dos números primos. Não há coátomos. O elemento máximo é 0 . Com a ordem $\unrhd$ (e com a usual também), o subconjunto $C_{n}=\left\{n^{k} \mid k \in \mathbb{N}^{*}\right\}$ de $\mathbb{N}$ é uma cadeia. 


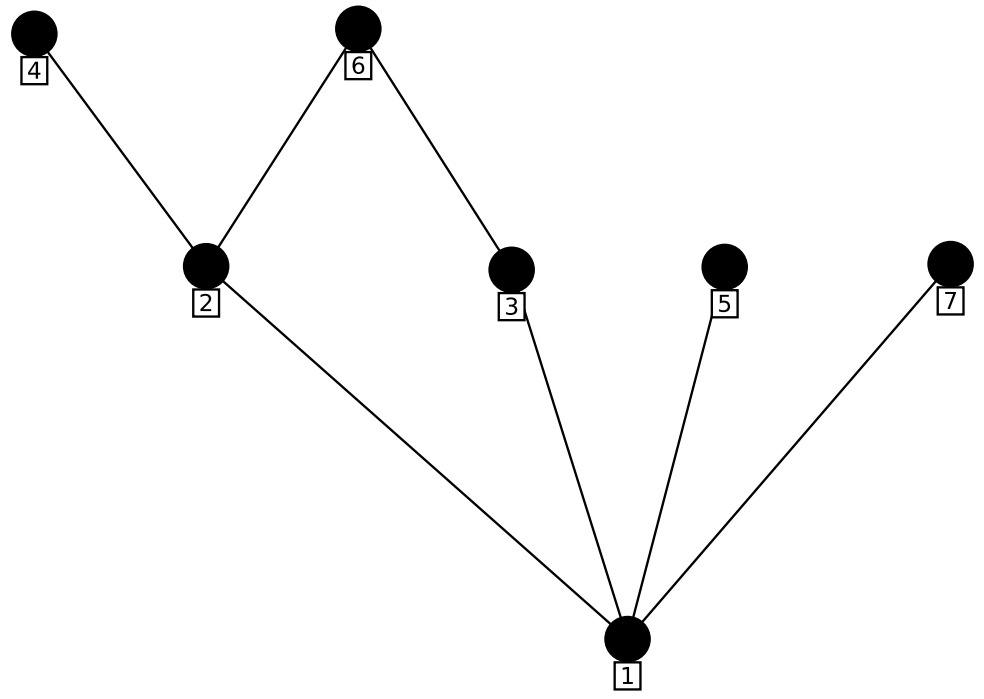

(a) Sub-ordem de $\langle\mathbb{N}, \unrhd\rangle$.

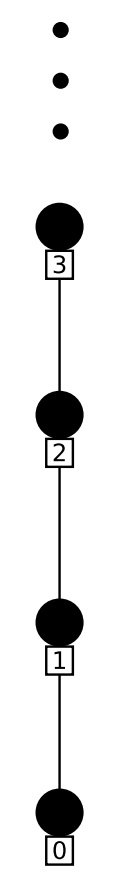

(b) $\langle\mathbb{N}, \leq\rangle$.

Figura 2.10: Representações de ordens parciais.

\subsubsection{Reticulados completos e famílias}

Se um conjunto parcialmente ordenado $L$ satisfizer a seguinte condição: para todo $S \subseteq L$, existe $\sup S$ e inf $S$, dizemos que $L$ é um reticulado completo.

Nem todo reticulado é completo. O seguinte subconjunto da cadeia dos reais é tal que $S^{u}$ é vazio (e portanto não possui elemento mínimo): $[0,+\infty)$. No entanto, todo intervalo fechado $[a, b]$ da reta real torna-se um reticulado completo, quando equipado com a relação de ordem usual. A cadeia dos racionais também não é um reticulado completo: o conjunto $S=\left\{x \in \mathbb{Q} \mid x^{2}<2\right\}$ possui limitantes superiores mas $S^{u}$ não possui elemento mínimo.

Uma família indexada de elementos de $S$ (ou simplesmente, familia) é uma função que associa um elemento $A_{i} \in S$ para cada índice $i \in I$. Denotamos isto por $\left\{A_{i}\right\}_{i \in I}$, e chamamos cada $A_{i}$ de membro da família. Neste capítulo, $S$ será frequentemente um reticulado completo ou o conjunto das partes de um conjunto fixado (tipicamente, conjunto de objetos ou atributos). No entanto, no decorrer deste texto, em particular, durante o Capítulo 7, tomaremos famílias de grafos, de maneira que, naquele contexto, $S$ deve ser entendido como uma classe.

Seja $L$ um reticulado completo. Então podemos tomar, em $L$, supremos e ínfimos sobre conjuntos arbitrários, de maneira que as operações binárias $\vee: L \times L \rightarrow L$ e $\wedge: L \times L \rightarrow L$ induzem funções de $\mathcal{P}(L)$ em $L: \bigvee S=\sup S$ e $\bigwedge S=\inf S$. Em particular, quando $S$ for uma família $S=\left\{A_{i}\right\}_{i \in I}$, com cada $A_{i} \in L$, denotamos o supremo

$$
\bigvee\left\{A_{i} \mid i \in I\right\} \text { simplesmente por } \bigvee_{i \in I} A_{i}
$$


Todo reticulado completo $L$ possui elemento máximo e mínimo, dados, respectivamente, por $\bigvee L$ e $\wedge L$. O outro caso extremo de supremos e ínfimos de $S \subseteq L$ é quando $S=\emptyset$. Em um conjunto parcialmente ordenado qualquer, temos que $\emptyset^{u}=\emptyset^{l}=L$ diretamente da definição, por vacuidade. Em palavras: o conjunto de limitantes superiores (inferiores) do vazio é todo o conjunto ordenado. Logo, em um reticulado completo, $\bigwedge \emptyset=\top$ e $\bigvee \emptyset=\perp$, já que as operações tomam, respectivamente, o maior limitante inferior e o menor limitante superior.

Supremo e ínfimo densidades Dizemos que um subconjunto $S$ de $L$ é supremo-denso (em $L$ ) se e só se para todo $x \in L$ existe $Q \subseteq S$ tal que $\bigvee Q=x$. Equivalentemente, $S$ é supremo-denso se e somente se $\{\bigvee Q \mid Q \subseteq S\}=L$, isto é, tomar o supremo de cada subconjunto de $S$ gera os elementos de $L$.

Definimos também o conceito dual: $S \subseteq L$ é ínfimo-denso (em $L$ ) se e somente se, todo elemento $x \in L$ pode ser escrito como o ínfimo $\wedge Q$ de um subconjunto $Q$ de $S$. Naturalmente, a ínfimo densidade de $S \subseteq L$ equivale a $\{\bigwedge Q \mid Q \subseteq S\}=L$.

\subsubsection{Exemplos}

O exemplo dado do conjunto das partes de $S$ é também um exemplo de reticulado completo. O supremo de uma família arbitrária de subconjuntos de $S$ é dado pela união desta família, enquanto que o ínfimo é dado pela intersecção.

Um conjunto supremo-denso de $\mathcal{P}(S)$ é o conjunto formado pelos seus átomos, isto é, pelos subconjuntos unitários de $S$. De fato, dado $A \in \mathcal{P}(S)$, se $A$ for vazio, então $A=\bigvee \emptyset$. Se $A$ não for vazio, claramente $A$ é a união de subconjuntos unitários de $S$. Se $S$ for finito, o conjunto formado pelos coátomos de $\mathcal{P}(S)$, isto é, os subconjuntos de cardinalidade $|S|-1$, é ínfimo-denso, por um argumento análogo.

$\operatorname{Em}\langle\mathbb{N}, \unrhd\rangle$, um conjunto supremo-denso é o formado por todas as cadeias de potências de números primos, isto é, o conjunto $\bigcup_{\text {p primo }} C_{p}$, onde $C_{p}=\left\{p^{k} \mid k \in \mathbb{N}^{*}\right\}$. Para ver este fato, basta lembrar que todo natural $n \geq 2$ pode ser escrito como produto de potências de primos $p_{1}^{k_{1}} p_{2}^{k_{2}} \cdots p_{\alpha}^{k_{\alpha}}$, e tal produto é o $m m c$ (generalizado) de $S=\left\{p_{1}^{k_{1}}, p_{2}^{k_{2}}, \ldots, p_{\alpha}^{k_{\alpha}}\right\}$, e então $n=\bigvee S$; para $n=0$ e $n=1$, basta notar que estes são os elementos máximo e mínimo, e portanto podem ser expressos como $\bigvee \mathbb{N}$ e $\bigvee \emptyset$, respectivamente.

\subsubsection{Preservação de ordem e homomorfismos}

Considere dois conjuntos parcialmente ordenados $P$ e $Q$. Por definição, uma função $\varphi: P \rightarrow Q$ preserva ordem se e somente se $x \leq y$ implica que $\varphi(x) \leq \varphi(y)$ para todo $x, y \in P$. A função $\varphi: P \rightarrow Q$ é dita um isomorfismo de ordem se $\varphi$ é bijetora e vale que $x \leq y$ se e só se $\varphi(x) \leq \varphi(y)$ para todo $x, y \in P$.

Para reticulados $L_{1}$ e $L_{2}$, podemos definir homomorfismos: uma função $\varphi: L_{1} \rightarrow L_{2}$ é dita um homomorfismo de reticulados se

$$
\begin{aligned}
& \varphi(x \wedge y)=\varphi(x) \wedge \varphi(y) \quad \mathrm{e} \\
& \varphi(x \vee y)=\varphi(x) \vee \varphi(y),
\end{aligned}
$$


para todo $x, y \in L_{1}$. Um homomorfismo de reticulados bijetor é dito um isomorfismo de reticulados.

Usaremos o seguinte resultado básico que pode ser encontrado em [DP02]. Em particular, este resultado garante que não existe a possibilidade de existir um isomorfismo de ordem entre dois reticulados, e não existir um isomorfismo de reticulados entre eles.

Proposição 2.4. Sejam $L_{1}$ e $L_{2}$ dois reticulados. São equivalentes:

i) Existe um isomorfismo de ordem entre $L_{1}$ e $L_{2}$.

ii) Existe um isomorfismo de reticulados entre $L_{1}$ e $L_{2}$.

iii) Existem funções $\varphi: L_{1} \rightarrow L_{2}$ e $\psi: L_{2} \rightarrow L_{1}$, ambas que preservam ordem, tais que $\varphi$ e $\psi$ são mutuamente inversas, isto é, $\varphi(\psi(x))$ e $\psi(\varphi(x))$ são funções identidade.

Quando existir um isomorfismo de ordem (e portanto, de reticulados), entre dois reticulados $L_{1}$ e $L_{2}$, denotaremos este fato por $L_{1} \cong L_{2}$.

Uma outra proposição, demonstrada na mesma referência, e que usaremos é o que segue. Na realidade, usamos apenas a conclusão mais fraca presente no final do enunciado. Apresentamos o fato desta maneira pois acreditamos que desta maneira há maior contextualização. Além disso, o seu conteúdo é bastante natural.

Proposição 2.5. Se $P$ e $Q$ forem dois conjuntos ordenados isomorfos, isto é, se existir isomorfismo de ordem $\varphi: P \rightarrow Q$, então $\varphi$ preserva supremos existentes, isto é, para cada $S \subseteq P$ tal que existe $\bigvee S$, existe também $\bigvee \varphi(S)$. Ademais, $\bigvee \varphi(S)=\varphi(\bigvee S)$. De maneira dual, $\varphi$ preserva infimos existentes. Em particular, se $P$ e $Q$ forem reticulados completos, então vale que $\varphi(\bigvee S)=\bigvee \varphi(S)$ e $\varphi(\bigwedge S)=\bigwedge \varphi(S)$, para todo $S \subseteq P$.

\subsection{Dualidade entre extensões e intenções}

Com o auxílio de algumas das definições feitas na seção anterior, estamos aptos a mostrar o caráter dual apresentado pelas extensões e intenções de um contexto. Considere uma ordem parcial qualquer $\langle P, \leq\rangle$. A ordem parcial dual de $\langle P, \leq\rangle$, é denotada por $\langle P, \geq\rangle$ ou $P^{\partial}$, e difere da ordem parcial original apenas pela relação de ordem, que é definida da maneira esperada a partir da original: $x \geq y \Leftrightarrow y \leq x$.

Seja $\mathbb{K}$ um contexto. Definimos como $\operatorname{Ext}(\mathbb{K})$ e $\operatorname{Int}(\mathbb{K})$, respectivamente, os conjuntos de extensões e de intenções de $\mathbb{K}$. Quando não estão explícitas as ordens parciais sobre $\operatorname{Ext}(\mathbb{K})$ e $\operatorname{Int}(\mathbb{K})$, deve-se subentender que cada ordem em questão é a relação de inclusão $\subseteq$.

O conjunto de conceitos $\mathfrak{B}(\mathbb{K})$, sendo formado por pares, pode ser projetado em cada uma das duas coordenadas, dando origem ao conjunto de extensões e ao conjunto de intenções. Mais formalmente, assim escrevemos essas projeções:

$$
\begin{aligned}
\pi_{1}: \mathfrak{B}(\mathbb{K}) & \rightarrow \operatorname{Ext}(\mathbb{K}) & \pi_{2}: \mathfrak{B}(\mathbb{K}) & \rightarrow \operatorname{Int}(\mathbb{K}) \\
(A, B) & \mapsto A & (A, B) & \mapsto B .
\end{aligned}
$$

Claramente, $\pi_{1}$ e $\pi_{2}$ são sobrejetoras. Além disso, como a extensão de um conceito determina sua intenção e vice-versa, temos que cada uma das funções $\pi_{1}$ e $\pi_{2}$ é injetora. Ambas funções são, portanto, bijetoras. 
Lembramos que a ordem parcial sobre conceitos, $\mathfrak{B}(\mathbb{K})$, foi definida em (2.1) de maneira a herdar a relação $\subseteq$ sobre $\operatorname{Ext}(\mathbb{K})$. Então, a função $\pi_{1}$ é um isomorfismo de ordem e, portanto, de reticulados. Por outro lado, mostramos em (2.2) que há uma definição equivalente, a saber, a de herdar a relação $\supseteq$ sobre $\operatorname{Int}(\mathbb{K})$. Essas observações implicam que um reticulado de conceitos é sempre isomorfo ao reticulado de extensões: $\mathfrak{B}(\mathbb{K}) \cong\langle\operatorname{Ext}(\mathbb{K}), \subseteq\rangle$, assim como isomorfo ao dual do reticulado de intenções, isto é: $\mathfrak{B}(\mathbb{K}) \cong\langle\operatorname{Int}(\mathbb{K}), \supseteq\rangle$. Em vez de "isomorfo ao dual", é comum que se diga anti-isomorfo. Assim, dizemos que um reticulado de conceitos é sempre anti-isomorfo ao seu reticulado de intenções, e que a função $\pi_{2}$ é um anti-isomorfismo de reticulados.

Claramente, o dual de $P^{\partial}$ é novamente a ordem parcial $P$. Além disso, a relação de isomorfismo é transitiva e respeitada pela aplicação de ${ }^{\partial}$ (ou seja: $P \cong Q \Leftrightarrow P^{\partial} \cong Q^{\partial}$ ). Consequentemente, os reticulados de extensões e de intenções são sempre anti-isomorfos entre si. No exemplo do subcontexto dos vertebrados, temos tais reticulados anti-isomorfos retratados na Figura 2.11.

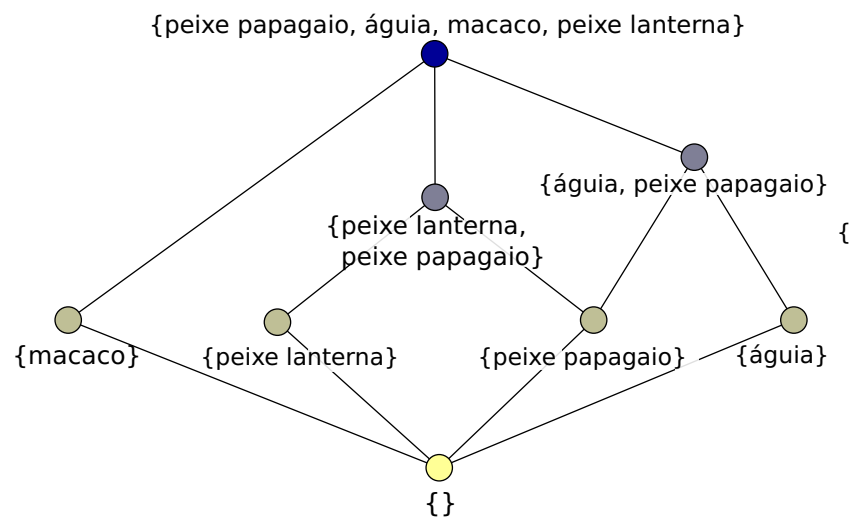

$\langle\operatorname{Ext}(\mathbb{K}), \subseteq\rangle$

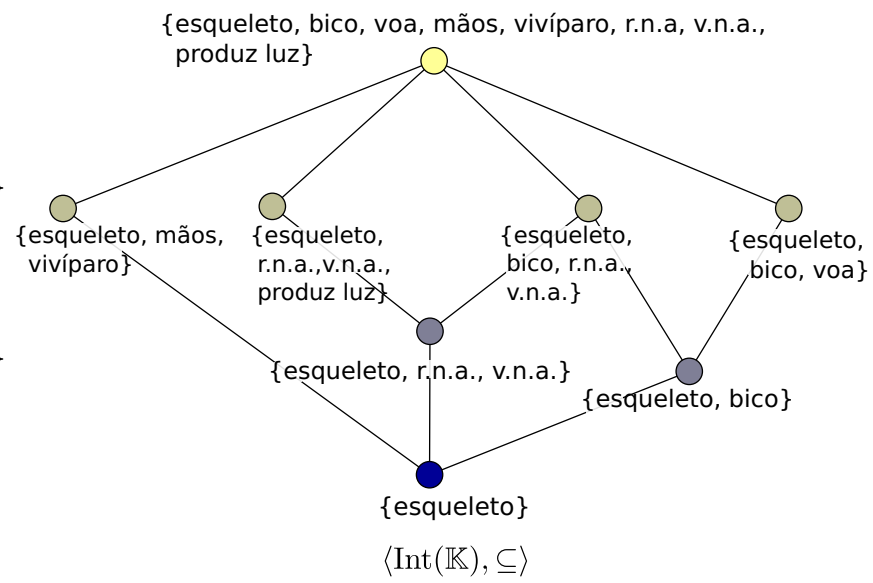

Figura 2.11: Reticulados de extensões e de intenções do subcontexto dos vertebrados.

O algoritmo de Nourine e Raynaud para a determinação do diagrama de Hasse de $\underline{\mathfrak{B}}(\mathbb{K})$, a ser exposto na seção 6.2 do Capítulo 6, se vale desta dualidade.

\subsection{Teorema fundamental}

Os Teoremas 2.7 e 2.8 e o Corolário 2.9 são resultados que caracterizam os reticulados de conceitos. Eles foram apresentados e demonstrados por Wille [Wil82], mas de maneira compilada, em apenas um teorema, constituindo o que é conhecido como teorema fundamental da FCA. Nossa apresentação é mais próxima à apresentação de Davey e Priestley [DP02]. Antes de chegarmos neste resultado, veremos a demonstração do Lema 2.6, bastante semelhante à lei de de Morgan, que prova que a derivação de uniões é a intersecção das derivações. Este lema será bastante útil para a demonstração dos Teoremas 2.7 e 2.8. 
Lema 2.6. Sejam $(G, M, I)$ um contexto e $\left\{A_{t}\right\}_{t \in T}$ uma familia indexada de subconjuntos de G. Então:

$$
\left(\bigcup_{t \in T} A_{t}\right)^{\prime}=\bigcap_{t \in T} A_{t}^{\prime}
$$

Uma igualdade análoga segue para conjuntos de atributos.

Prova. Seja $m \in\left(\bigcup_{t \in T} A_{t}\right)^{\prime}$. Da definição de derivação, temos que

$$
m \in\left(\bigcup_{t \in T} A_{t}\right)^{\prime} \Leftrightarrow\left(\forall g \in \bigcup_{t \in T} A_{t}\right) g I m
$$

Assim como:

$$
\left(\forall g \in \bigcup_{t \in T} A_{t}\right) g I m \Leftrightarrow(\forall t \in T)\left(\forall g \in A_{t}\right) g I m,
$$

pois podemos quantificar os elementos de cada conjunto $A_{t}$ por vez. A sentença do lado direito claramente equivale a

$$
(\forall t \in T) m \in A_{t}^{\prime} \Leftrightarrow m \in \bigcap_{t \in T} A_{t}^{\prime}
$$

O próximo resultado mostra que toda hierarquia de conceitos é um reticulado completo, e provê expressões para o cálculo de ínfimos e supremos arbitrários nesta estrutura.

Teorema 2.7. Para todo contexto $(G, M, I)$, a hierarquia de conceitos $\underline{\mathfrak{B}}(G, M, I)$ é um reticulado completo, no qual ínfimos e supremos são dados por

$$
\begin{aligned}
& \bigwedge_{j \in J}\left(A_{j}, B_{j}\right)=\left(\bigcap_{j \in J} A_{j},\left(\bigcup_{j \in J} B_{j}\right)^{\prime \prime}\right) e \\
& \bigvee_{j \in J}\left(A_{j}, B_{j}\right)=\left(\left(\bigcup_{j \in J} A_{j}\right)^{\prime \prime}, \bigcap_{j \in J} B_{j}\right),
\end{aligned}
$$

para uma familia de conceitos $\left\{\left(A_{j}, B_{j}\right)\right\}_{j \in J}$ qualquer de $\underline{\mathfrak{B}}(G, M, I)$.

Prova. Seja $\left\{\left(A_{j}, B_{j}\right)\right\}_{j \in J}$ uma família de conceitos de $\underline{\mathfrak{B}}(G, M, I)$. Definimos

$$
(A, B)=\left(\bigcap_{j \in J} A_{j},\left(\bigcup_{j \in J} B_{j}\right)^{\prime \prime}\right) .
$$

Vamos provar que $(A, B)$ é o maior limitante inferior da família dada. Para isso, mostramos primeiramente que $(A, B)$ é um conceito. Como $\left\{\left(A_{j}, B_{j}\right)\right\}_{j \in J}$ é uma família de conceitos, segue que $A_{j}=B_{j}^{\prime}$ para todo $j \in J$. Logo,

$$
\begin{aligned}
(A, B) & =\left(\bigcap_{j \in J} B_{j}^{\prime}, \quad\left(\bigcup_{j \in J} B_{j}\right)^{\prime \prime}\right) \\
& =\left(\left(\bigcup_{j \in J} B_{j}\right)^{\prime},\left(\bigcup_{j \in J} B_{j}\right)^{\prime \prime}\right) \quad \text { (pelo Lema 2.6) }
\end{aligned}
$$


o que significa que a segunda coordenada deste par é a derivação da primeira coordenada. Pelo item (iii) da Proposição 2.3, sobre derivação tripla, segue que $\left(\bigcup_{j \in J} B_{j}\right)^{\prime \prime \prime}=\left(\bigcup_{j \in J} B_{j}\right)^{\prime}$, e portanto $(A, B)$ é um conceito.

É claro que $(A, B)$ é um limitante inferior de $\left\{\left(A_{j}, B_{j}\right)\right\}_{j \in J}$, já que

$$
(\forall j \in J) A_{j} \supseteq \bigcap_{k \in J} A_{k}=A,
$$

e portanto, pela definição de hierarquia conceitual em (2.1), temos que $(A, B) \leq\left(A_{j}, B_{j}\right)$, para todo $j \in J$. Também vale que $(A, B)$ é o maior dos limitantes inferiores. De fato, considere um conceito $\left(A^{*}, B^{*}\right) \in(G, M, I)$ que seja um limitante inferior de $\left\{\left(A_{j}, B_{j}\right)\right\}_{j \in J}$. Logo,

$$
(\forall j \in J) A^{*} \subseteq A_{j} \Rightarrow A^{*} \subseteq \bigcap_{j \in J} A_{j}=A,
$$

e, portanto, segue que $\left(A^{*}, B^{*}\right) \leq(A, B)$, novamente pela definição de hierarquia conceitual.

Com relação ao supremo, definimos

$$
(A, B)=\left(\left(\bigcup_{j \in J} A_{j}\right)^{\prime \prime}, \bigcap_{j \in J} B_{j}\right) .
$$

Novamente $(A, B)$ é um conceito, pois

$$
\begin{aligned}
(A, B) & =\left(\left(\bigcup_{j \in J} A_{j}\right)^{\prime \prime}, \bigcap_{j \in J} A_{j}^{\prime}\right) \\
& =\left(\left(\bigcup_{j \in J} A_{j}\right)^{\prime \prime},\left(\bigcup_{j \in J} A_{j}\right)^{\prime}\right) \quad \text { (pelo Lema 2.6). }
\end{aligned}
$$

Usando novamente a propriedade da derivação tripla, segue que $(A, B)$ é um conceito. Quando utilizamos a definição alternativa da hierarquia conceitual (2.1), que estabelece que $\left(A_{1}, B_{1}\right) \leq\left(A_{2}, B_{2}\right) \Leftrightarrow B_{1} \supseteq B_{2}$, e usamos a mesma argumentação usada para estabelecer (2.3), segue que $(A, B)$ é o menor limitante superior de $\left\{\left(A_{j}, B_{j}\right)\right\}_{j \in J}$.

O Teorema 2.7 comprova que, para qualquer contexto $(G, M, I)$, a estrutura $\underline{\mathfrak{B}}(G, M, I)$ é um reticulado completo. Naturalmente, a recíproca se põe em questão. Será que, para todo reticulado completo $L$ existe um contexto $(G, M, I)$ tal que $\underline{B}(G, M, I) \cong L$ ? A resposta a essa pergunta é sim, como veremos no Corolário 2.9. Em outras palavras, podemos dizer que todo reticulado completo $L$ se realiza como reticulado de conceitos de algum contexto formal. No entanto, veremos que esta realização, em geral, não é única. Há diversos contextos cujos reticulados são isomorfos e, inclusive, é possível que dois contextos com "tamanhos" muito diferentes possuam reticulados isomorfos. Isto será melhor ilustrado após a demonstração do Corolário 2.10.

O teorema a seguir caracteriza as realizações de um reticulado completo $L$ como reticulado de conceitos. A seguinte notação, bastante comum, será utilizada em seu enunciado e demonstração: para uma função qualquer $f$, de domínio $X$, e um conjunto $S \subseteq X$, o símbolo $f(S)$ denota a união $\bigcup_{s \in S} f(s)$. 
Teorema 2.8. Sejam $L$ um reticulado completo e $(G, M, I)$ um contexto formal. Então, vale que $L$ é isomorfo a $\mathfrak{B}(G, M, I)$ se e somente se existirem funçôes $\gamma: G \rightarrow L$ e $\mu: M \rightarrow L$ tais que $\gamma(G)$ é supremo-denso em $L, \mu(M)$ é infimo-denso em $L$ e $g I m \Leftrightarrow \gamma(g) \leq \mu(m)$, para todo $g \in G, m \in M$.

Prova. $(\Rightarrow)$ : Sejam $L \cong \mathfrak{B}(G, M, I)$ e $\varphi: \mathfrak{B}(G, M, I) \rightarrow L$ um isomorfismo de reticulados (e portanto, também isomorfismo de ordem). Mostraremos que existem funções com as propriedades citadas. Defina as funções

$$
\begin{aligned}
\gamma: G & \rightarrow L & \mu: M & \rightarrow L \\
g & \mapsto \varphi\left(\left(\{g\}^{\prime \prime},\{g\}^{\prime}\right)\right) & m & \mapsto \varphi\left(\left(\{m\}^{\prime},\{m\}^{\prime \prime}\right)\right) .
\end{aligned}
$$

A partir de agora e até o fim desta prova, escreveremos simplesmente $g^{\prime}$ em vez de $\{g\}^{\prime}$ e idem para $\{m\}^{\prime}$. Mostraremos que $\gamma(G)$ é supremo-denso em $L$. Para tanto, fixamos $x \in L$ e mostraremos que existe $S \subseteq G$ tal que $\bigvee_{s \in S} \gamma(s)=x$.

Seja

$$
(A, B)=\varphi^{-1}(x) .
$$

Tomamos $S=A$, e mostraremos que $\bigvee_{g \in A} \gamma(g)=x$. Vale que

$$
\begin{aligned}
\bigvee_{g \in A} \gamma(g) & \left.=\bigvee_{g \in A} \varphi\left(\left(g^{\prime \prime}, g^{\prime}\right)\right) \quad \text { (pela definição de } \gamma\right) \\
& =\varphi\left(\bigvee_{g \in A}\left(g^{\prime \prime}, g^{\prime}\right)\right) \quad \text { (pela Proposição 2.5, pois } \varphi \text { é isomorfismo). }
\end{aligned}
$$

Note que o supremo $\bigvee_{g \in A}\left(g^{\prime \prime}, g^{\prime}\right)$ é calculado sobre a estrutura $\underline{\mathfrak{B}}(G, M, I)$. Do Teorema 2.7, temos que

$$
\begin{array}{rlr}
\bigvee_{g \in A}\left(g^{\prime \prime}, g^{\prime}\right) & =\left(\left(\bigcup_{g \in A} g^{\prime \prime}\right)^{\prime \prime}, \bigcap_{g \in A} g^{\prime}\right) \\
& =\left(\left(\bigcap_{g \in A} g^{\prime \prime \prime}\right)^{\prime}, \bigcap_{g \in A} g^{\prime}\right) \quad \text { (pelo Lema 2.6) } \\
& =\left(\left(\bigcap_{g \in A} g^{\prime}\right)^{\prime}, \bigcap_{g \in A} g^{\prime}\right) \quad \text { (pela Proposição 2.3, item (iii)) } \\
& =\left(\left(\bigcup_{g \in A}\{g\}\right)^{\prime \prime},\left(\bigcup_{g \in A}\{g\}\right)^{\prime}\right) \quad(\text { pelo Lema 2.6) } \\
& =\left(A^{\prime \prime}, A^{\prime}\right) .
\end{array}
$$

Como $(A, B) \in \mathfrak{B}(G, M, I)$, segue pela Proposição 2.2 que $A^{\prime \prime}=A$ e $A^{\prime}=B$. Portanto, $\bigvee_{g \in A}\left(g^{\prime \prime}, g^{\prime}\right)=(A, B)$. Assim, vale que $\varphi\left(\bigvee_{g \in A}\left(g^{\prime \prime}, g^{\prime}\right)\right)=\varphi((A, B))=x$, onde a última 
igualdade segue de (2.4). Concluímos que $\gamma(G)$ é supremo-denso em $L$. A ínfimo-densidade de $\mu$ segue de maneira similar.

Agora, mostraremos a equivalência entre $g \operatorname{Im}$ e $\gamma(g) \leq \mu(m)$ :

$$
\begin{array}{rlrl}
\gamma(g) \leq \mu(m) & \Leftrightarrow \varphi\left(\left(g^{\prime \prime}, g^{\prime}\right)\right) \leq \varphi\left(\left(m^{\prime}, m^{\prime \prime}\right)\right) & \text { (pelas definições de } \gamma \text { e } \mu \text { ) } \\
& \Leftrightarrow\left(g^{\prime \prime}, g^{\prime}\right) \leq\left(m^{\prime}, m^{\prime \prime}\right) & \text { (pois } \varphi \text { é isomorfismo de ordem) } \\
& \Leftrightarrow\{g\}^{\prime \prime} \subseteq\{m\} & \text { (hierarquia conceitual, def. em (2.1)) } \\
& \Leftrightarrow\{g\}^{\prime} \supseteq\{m\} & \text { (itens (iv) e (ii'), Proposição 2.3) } & \\
& \Leftrightarrow m \in\{g\}^{\prime} \Leftrightarrow g I m . &
\end{array}
$$

$(\Leftarrow)$ : Sejam $L$ um reticulado completo, $(G, M, I)$ um contexto e $\gamma: G \rightarrow L, \mu: M \rightarrow L$ funções com as propriedades do enunciado. Primeiramente mostraremos que:

$$
(\forall A \subseteq G)(\forall m \in M) m \in A^{\prime} \Leftrightarrow \bigvee_{g \in A} \gamma(g) \leq \mu(m)
$$

De fato,

$$
\begin{array}{rlrl}
m \in A^{\prime} & \Leftrightarrow(\forall g \in A) g I m & \\
& \Leftrightarrow(\forall g \in A) \gamma(g) \leq \mu(m) & \text { (por hipótese, } g \operatorname{Im} \Leftrightarrow \gamma(g) \leq \mu(m)) \\
& \Leftrightarrow \mu(m) \text { é um limitante superior de }\{\gamma(g)\}_{g \in A} \\
& \Leftrightarrow \bigvee_{g \in A} \gamma(g) \leq \mu(m) \quad \text { (pois } \bigvee_{g \in A} \gamma(g) \text { é o menor dos lim. superiores). }
\end{array}
$$

De maneira dual, mostraremos agora que:

$$
(\forall B \subseteq M)(\forall g \in G) g \in B^{\prime} \Leftrightarrow \gamma(g) \leq \bigwedge_{m \in B} \mu(m) .
$$

De fato,

$$
\begin{array}{rlr}
g \in B^{\prime} & \Leftrightarrow(\forall m \in B) g I m \\
& \Leftrightarrow(\forall m \in B) \gamma(g) \leq \mu(m) \quad \text { (por hipótese, } g \operatorname{Im} \Leftrightarrow \gamma(g) \leq \mu(m)) \\
& \Leftrightarrow \gamma(g) \text { é um limitante inferior de }\{\mu(m)\}_{m \in B} \\
& \Leftrightarrow \gamma(g) \leq \bigwedge_{m \in B} \mu(m) \quad \text { (pois } \bigwedge_{m \in B} \mu(m) \text { é o maior dos lim. inferiores). }
\end{array}
$$

Definiremos funções $\varphi: \underline{\mathfrak{B}}(G, M, I) \rightarrow L$ e $\psi: L \rightarrow \underline{\mathfrak{B}}(G, M, I)$, ambas que preservam ordem, e tais que uma é a inversa da outra. Pela Proposição 2.4, isto estabelecerá que $L$ é isomorfo a $\mathfrak{B}(G, M, I)$, e encerrará a demonstração. Para $x \in L$, sejam

$$
\begin{aligned}
& A_{x}=\{g \in G \mid \gamma(g) \leq x\} \text { e } \\
& B_{x}=\{m \in M \mid x \leq \mu(m)\} .
\end{aligned}
$$

Vamos mostrar que

$$
(\forall x \in L) \bigvee_{g \in A_{x}} \gamma(g)=x
$$


De fato, como $\gamma(G)$ é supremo-denso em $L$ (por hipótese), temos que existe $\gamma(Q) \subseteq \gamma(G)$ tal que $\bigvee \gamma(Q)=x$. Claramente, o conjunto $\gamma(Q)$ não pode conter um elemento incomparável ou estritamente maior do que $x$, pois $\bigvee \gamma(Q)=x$. Logo, $Q$ está contido em $A_{x}$. Portanto, $\bigvee_{g \in A_{x}} \gamma(g) \geq \bigvee_{g \in Q} \gamma(g)=x$

Por outro lado, a delimitação $\bigvee_{g \in A_{x}} \gamma(g) \leq x$ é clara, bastando observar que a definição de $A_{x}$ exige que $x$ seja um limitante superior de cada $\gamma(g) \operatorname{com} g \in A_{x}$. Assim, mostramos (2.7). Um argumento dual estabelece que

$$
(\forall x \in L) \bigwedge_{m \in B_{x}} \mu(m)=x
$$

Queremos provar que, para todo $x \in L$, o par $\left(A_{x}, B_{x}\right)$ é um conceito de $(G, M, I)$. Seja $m \in M$. Temos que

$$
\begin{aligned}
m \in A_{x}^{\prime} & \Leftrightarrow \bigvee_{g \in A_{x}} \gamma(g) \leq \mu(m) \\
& \Leftrightarrow x \leq \mu(m) \\
& \Leftrightarrow m \in B_{x}
\end{aligned}
$$

(pela definição de $B_{x}$ ).

Logo, $A_{x}^{\prime}=B_{x}$. Seja $g \in G$. Temos que

$$
\begin{array}{rlr}
g \in B_{x}^{\prime} & \Leftrightarrow \gamma(g) \leq \bigwedge_{m \in B_{x}} \mu(m) & \text { (por }(2.6)) \\
& \Leftrightarrow \gamma(g) \leq x & \\
& \Leftrightarrow g \in A_{x} & \text { (por }(2.8))
\end{array}
$$

Assim, $B_{x}^{\prime}=A_{x}$, e portanto, $\left(A_{x}, B_{x}\right)$ é um conceito, para todo $x \in L$. Conforme mencionamos, iremos definir duas funções que preservam ordem e que são mutuamente inversas. A primeira delas a ser definida é $\psi$ :

$$
\begin{aligned}
\psi: L & \longrightarrow \mathfrak{B}(G, M, I) \\
x & \longmapsto\left(A_{x}, B_{x}\right) .
\end{aligned}
$$

Não é difícil ver que $\psi$ preserva ordem: $x \leq y$ implica que $A_{x} \subseteq A_{y}$, diretamente da definição de $A_{x}$. Ou seja, $\left(A_{x}, B_{x}\right) \leq\left(A_{y}, B_{y}\right)$ em $\underline{\mathfrak{B}}(G, M, I)$. Definimos também uma função $\varphi$ :

$$
\begin{aligned}
\varphi: \underline{\mathfrak{B}}(G, M, I) & \longrightarrow L \\
(A, B) & \longmapsto \bigvee_{g \in A} \gamma(g)
\end{aligned}
$$

A função $\varphi$ preserva ordem, já que

$$
\begin{aligned}
\left(A_{1}, B_{1}\right) \leq\left(A_{2}, B_{2}\right) \Leftrightarrow A_{1} \subseteq A_{2} & \Rightarrow \gamma\left(A_{1}\right) \subseteq \gamma\left(A_{2}\right) \\
& \left.\Rightarrow \bigvee_{g \in A_{1}} \gamma(g) \leq \bigvee_{g \in A_{2}} \gamma(g) \quad \text { (monotonicidade crescente de } \bigvee\right)
\end{aligned}
$$


Resta mostrarmos que $\varphi$ e $\psi$ são mutuamente inversas. Seja $x \in L$. Temos que:

$$
\begin{aligned}
\varphi(\psi(x))=\varphi\left(\left(A_{x}, B_{x}\right)\right) & =\bigvee_{g \in A_{x}} \gamma(g) & \quad(\text { pelas definições de } \psi \text { e } \varphi) \\
& =x & (\text { por }(2.7)) .
\end{aligned}
$$

Agora, seja $(A, B) \in \underline{\mathfrak{B}}(G, M, I)$. Calcularemos $\psi(\varphi((A, B)))$, a fim de mostrarmos que isto vale $(A, B)$. Temos que:

$$
\psi(\varphi((A, B)))=\psi\left(\bigvee_{g \in A} \gamma(g)\right) \quad(\text { pela definição de } \varphi)
$$

Definimos

$$
x=\bigvee_{g \in A} \gamma(g) .
$$

Queremos provar que $\psi(x)=(A, B)$. Pela definição de $\psi$, isto significa provarmos as igualdades $A_{x}=A$ e $B_{x}=B$. Inicialmente mostramos que $A \subseteq A_{x}$. De fato, para todo $g_{0} \in A$, claramente vale que $\gamma\left(g_{0}\right) \leq \bigvee_{g \in A} \gamma(g)$, e por (2.9), temos que $\bigvee_{g \in A} \gamma(g)=x$, e portanto $\gamma\left(g_{0}\right) \leq x$, o que implica que $g_{0} \in A_{x}$, pela definição de $A_{x}$. Então, segue que $A \subseteq A_{x}$. Agora, provaremos que $A_{x} \subseteq A$. Suponha que $g \in A_{x}$. Logo, $\gamma(g) \leq x$, e então

$$
\gamma(g) \leq x=\bigvee_{g \in A} \gamma(A)
$$

Aplicando (2.5), temos que:

$$
\left(\forall m \in A^{\prime}\right) \bigvee_{g \in A} \gamma(g) \leq \mu(m)
$$

Note primeiramente que $A^{\prime \prime}=A$, pois $(A, B) \in \mathfrak{B}(G, M, I)$ e a Proposição 2.2 garante que toda extensão é fechado de ". Aplicando transitividade em (2.10) e (2.11), concluímos que:

$$
\begin{aligned}
\left(\forall m \in A^{\prime}\right) \gamma(g) \leq \mu(m) & \Leftrightarrow\left(\forall m \in A^{\prime}\right) g I m & & \text { (por hipótese) } \\
& \Leftrightarrow g \in A^{\prime \prime} & & \text { (pela definição de derivação) } \\
& \Leftrightarrow g \in A & & \text { (pois } \left.A^{\prime \prime}=A\right) .
\end{aligned}
$$

Consequentemente, $A_{x} \subseteq A$ e portanto $A_{x}=A$. Como $\left(A_{x}, B_{x}\right)$ e $(A, B)$ são dois conceitos com a mesma extensão, segue que $B_{x}=B$. Logo, $\mathfrak{B}(G, M, I) \cong L$.

O corolário a seguir mostra que, para todo reticulado completo $\langle L, \leq\rangle$, existe um contexto que o realiza enquanto reticulado de conceitos. A saber, $\mathbb{K}=(L, L, \leq)$.

Corolário 2.9. Se $L$ for um reticulado completo, então $L \cong \mathfrak{B}(L, L, \leq)$.

Prova. Basta tomarmos $G=L, M=L$, a relação de incidência como a relação de ordem parcial associada a $L$ e as funções $\gamma$ e $\mu$ como a identidade $i d_{L}: L \rightarrow L$. As condições de supremo e ínfimo-densidade são trivialmente verificadas, assim como a condição sobre a relação de incidência, isto é, $g \operatorname{Im} \Leftrightarrow \gamma(g) \leq \mu(m)$. 
O próximo corolário mostra que existe uma família de contextos arbitrariamente grandes cujos reticulados de conceitos possuem tamanho exponencial em função do tamanho dos contextos.

Corolário 2.10. Para todo $k \in \mathbb{N}^{*}$, existe um contexto $\mathbb{K}^{k}=(G, M, I)$ com $|G|=|M|=k$ e cujo reticulado de conceitos é isomorfo ao reticulado booleano $\langle\mathcal{P}([k]), \subseteq\rangle$. Em particular, $|\mathfrak{B}(G, M, I)|=2^{k}$.

Prova. Seja $k \in \mathbb{N}^{*}$. Definimos que $G=M=[k]=\{1,2, \ldots, k\}$. Considere a seguinte ordenação dos átomos e coátomos de $\langle\mathcal{P}([k]), \subseteq\rangle$ :

$$
\begin{array}{ll}
\mathcal{A}_{i}=\{i\}, & \text { para } i=1,2, \ldots, k . \\
\mathcal{C}_{i}=[k] \backslash\{i\}, & \text { para } i=1,2, \ldots, k .
\end{array}
$$

Definimos a função $\gamma: G \rightarrow \mathcal{P}([k])$ dada por

$$
\gamma(i)=\mathcal{A}_{i} \text {, para todo } i \in G .
$$

Também definimos $\mu: M \rightarrow \mathcal{P}([k])$ dada por

$$
\mu(i)=\mathcal{C}_{i} \text {, para todo } i \in M .
$$

Desta maneira, $\gamma(G)$ é o conjunto de átomos e $\mu(M)$ é o conjunto de coátomos do reticulado booleano. Logo, $\gamma(G)$ e $\mu(M)$ são, respectivamente, supremo e ínfimo densos em $\mathcal{P}([k])$. Ao definirmos a relação de incidência $I$ como

$$
i I j \Leftrightarrow \mathcal{A}_{i} \subseteq \mathcal{C}_{j},
$$

para cada $i \in G$ e cada $j \in M$, temos que $i I j \Leftrightarrow \gamma(i) \subseteq \mu(j)$, isto é, a relação $I$ satisfaz a condição do Teorema 2.8. As condições sobre supremo-densidade de $\gamma(G)$ e ínfimo-densidade de $\mu(M)$ também estão satisfeitas e portanto $\mathfrak{B}(G, M, I) \cong\langle\mathcal{P}([k]), \subseteq\rangle$.

Convém observar a forma tabular do contexto assim definido. Sem dificuldades se observa que $\mathcal{A}_{i} \subseteq \mathcal{C}_{j}$ se e só se $i \neq j$. Então o contexto $(G, M, I)$ pode ser representado por uma tabela que apenas não possui cruzes na diagonal:

\begin{tabular}{|c|c|c|c|c|c|}
\hline $\mathbb{K}$ & 1 & 2 & 3 & $\ldots$ & $k$ \\
\hline 1 & & $\times$ & $\times$ & $\ldots$ & $\times$ \\
\hline 2 & $\times$ & & $\times$ & $\ldots$ & $\times$ \\
\hline 3 & $\times$ & $\times$ & & $\ldots$ & $\times$ \\
\hline$\vdots$ & $\vdots$ & $\vdots$ & & $\ddots$ & $\vdots$ \\
\hline$k$ & $\times$ & $\times$ & $\times$ & $\ldots$ & \\
\hline
\end{tabular}

Os grafos associados aos contextos $\mathbb{K}^{k}$, com valores de $k$ de 1 até 4 estão retratados na Figura 2.12. 
Dado um grafo $(V, E)$, dizemos que um conjunto $S \subseteq E$ é um emparelhamento se, para cada par de arestas distintas $e_{1}, e_{2} \in S$, cada ponta de $e_{1}$ é diferente de cada ponta de $e_{2}$. Se, adicionalmente, cada vértice $v \in V$ for ponta de alguma aresta $e \in S$, dizemos que $S$ é (um emparelhamento) perfeito.

Com relação aos contextos $\mathbb{K}^{k}$ construídos no Corolário 2.10, podemos dizer que cada grafo associado $\mathcal{G}\left(\mathbb{K}^{k}\right)$ é o grafo bipartido completo $K_{k, k}$, após sofrer a remoção de um emparelhamento perfeito qualquer. Esta família de grafos é conhecida como família dos grafos coroas. Para $k \in \mathbb{N}^{*}$, denotamos por $C G(2 k)$ o grafo coroa com $2 k$ vértices. Os grafos coroa com $k$ de 1 até 4 estão representados na Figura 2.12.

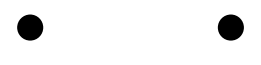

(a) $\mathrm{CG}(2)$

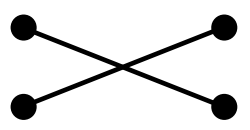

(b) $\mathrm{CG}(4)$

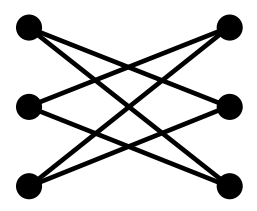

(c) $\mathrm{CG}(6)$

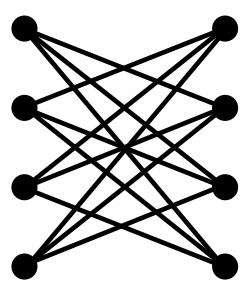

(d) $\mathrm{CG}(8)$

Figura 2.12: Grafos coroa $C G(2 k)$ com $k$ de 1 até 4.

Como já haviamos adiantado, um reticulado completo pode se realizar de diversas maneiras como um reticulado de conceitos. Podemos ilustrar este fato utilizando os Corolários 2.9 e 2.10. Considere o reticulado booleano $\mathcal{P}([k])$. Apesar do Corolário 2.9 garantir o isomorfismo $\mathcal{P}([k]) \cong \mathfrak{B}(\mathcal{P}([k]), \mathcal{P}([k]), \subseteq)$, o Corolário 2.10 mostra que $\mathcal{P}([k])$ é isomorfo ao reticulado de conceitos de um contexto muito menor: $\mathcal{P}([k]) \cong \mathfrak{B}([k],[k], \neq)$. 


\section{Capítulo 3}

\section{O problema da contagem de conceitos}

O conceito de solubilidade em tempo polinomial na teoria de complexidade computacional é de extrema importância para a ciência da computação, e muitas vezes norteia a noção de quais problemas podem ser resolvidos, em tempo hábil, por um processo computacional.

Neste trabalho, estamos particularmente interessados nos problemas pertencentes à classe de complexidade NP. O leitor interessado em uma abordagem introdutória precisa a esta classe é convidado a consultar [GJ79]. Os problemas em NP são problemas de decisão, como por exemplo: decidir se uma fórmula booleana possui atribuição que a satisfaça, decidir se um grafo possui um caminho hamiltoniano e etc. Se um problema $\Pi$, pertencente a NP, admitir uma solução de tempo polinomial, então dizemos que $\Pi$ pertence à classe $\mathbf{P}$.

Durante os Capítulos 5 e 6, serão abordados problemas de enumeração. Em contraste com os problemas de decisão, as soluções dos problemas de enumeração consistem em processos computacionais que listam ${ }^{1}$ todos os objetos possuindo certa característica: a listagem dos conceitos de um contexto formal, a listagem dos triângulos ${ }^{2}$ de um grafo e etc.

Os problemas de enumeração a serem tratados em tais capítulos servem de motivação para abordarmos um terceiro tipo de problema: os problemas de contagem. Neste capítulo, falaremos em especial sobre uma classe de problemas chamada \#P, a ser mais detalhadamente descrita na próxima seção.

\subsection{Problemas de contagem}

Os problemas de contagem que aqui nos interessam pertencem a uma classe de complexidade chamada \#P , introduzida por Leslie Valiant em [Val79a]. Esta classe é constituída pelos problemas de contagem associados aos problemas de NP, como por exemplo: contar quantas atribuições satisfazem uma fórmula booleana, contar quantos caminhos hamiltonianos um grafo possui e etc. Muitas vezes diremos somente problema de contagem para nos referirmos a um problema em \#P.

Assim como na classe NP, existem problemas em \#P que são completos [Val79a, Val79b]. Isto é, existem problemas de contagem para o qual todo problema de contagem pode ser redu-

\footnotetext{
${ }^{1}$ Os verbos "enumerar" e "encontrar" também são encontrados na literatura, apresentando o mesmo significado para o qual usamos a palavra "listar" aqui.

${ }^{2}$ Um triângulo é um conjunto de três vértices, dos quais dois a dois são adjacentes.
} 
zido, em tempo polinomial. Naturalmente, tais problemas são chamados de \#P-completos. Como demonstração de que os problemas \#P-completos são difíceis, é possível mostrar, de maneira totalmente elementar, que existem problemas de contagem que são pelo menos tão difíceis quanto os NP-completos. Considere, por exemplo ${ }^{3}$, o problema \#3-SAT:

\section{Problema \#3-SAT}

Entrada: Uma fórmula $\phi$ da forma $\bigwedge_{i=1}^{s} C_{i}$, onde cada $C_{i}$ é uma cláusula com três literais.

Saída: O número de atribuições das variáveis de $\phi$ que a satisfazem.

É evidente que a existência de um algoritmo de tempo polinomial para o problema descrito acima implica que existe um algoritmo, também de tempo polinomial, para o problema de decisão 3-SAT, e portanto implica que $\mathbf{P}=\mathbf{N P}$. Consequentemente, podemos considerar que \#3-SAT é um problema difícil, assim como todos os problemas \#P-completos.

De maneira geral, os problemas de contagem são pelo menos tão difíceis quanto sua versão original de decisão. Em alguns casos, as versões de contagem são muito mais difíceis: existem problemas \#P-completos associados a problemas em P. Por exemplo, considere o problema de decisão 2-SAT:

\section{Problema 2-SAT}

Entrada: Uma fórmula $\phi$ da forma $\bigwedge_{i=1}^{s} C_{i}$, onde cada $C_{i}$ é uma cláusula com dois literais.

Saída: Sim, se existir atribuição das variáveis de $\phi$ que a satisfazem e não caso contrário.

Este problema pode ser resolvido em tempo polinomial [Kro67]. No entanto, Valiant mostrou em [Val79b] que o \#2-SAT-MONótono, que é a restrição de \#2-SAT em que não há quaisquer negações em literais, é \#P-completo. Naturalmente, isso implica que o \#2-SAT é \#P-completo. Ainda em [Val79b], o autor mostra outro exemplo de problema em $\mathbf{P}$ cuja versão de contagem é difícil: o problema de decidir, dado um grafo bipartido, se este possui um emparelhamento perfeito.

\section{Um problema de contagem em Análise de Conceitos Formais}

Contextos que possuem um número muito grande de conceitos, como por exemplo, contextos associados aos grafos coroa $C G(2 k)$, não podem ter seus conceitos enumerados em tempo polinomial. Isso se deve ao fato da saída ser exponencialmente maior do que a entrada, e portanto, a própria devolução da saída, por parte do algoritmo, requer tempo exponencial nestes casos.

Tais contextos podem ser vistos como casos difíceis para os algoritmos que veremos nos Capítulos 5 e 6, e também como indesejáveis para aplicações interativas que serão exibidas

\footnotetext{
${ }^{3} \mathrm{O}$ simbolo $\bigwedge$ já foi utilizado por nós para denotar o maior dos limitantes inferiores, e aqui significa especificamente a operação de conjunção, isto é, "E lógico".
} 
durante o Capítulo 4. Uma solução para o problema da contagem de conceitos de um contexto, portanto, configuraria um processo computacional de interesse para a detecção de tais contextos. Mais formalmente, consideraremos o problema \#CONCEPT:

\section{Problema \#CONCEPT}

Entrada: Um contexto formal $(G, M, I)$

Saída: $|\mathfrak{B}(G, M, I)|$.

Note que a saída do problema \#CONCEPT é um número natural limitado superiormente ${ }^{4}$ por $2^{|G|+|M|}$ e que, em notação binária, isto pode ser representado usando espaço polinomial em $|G|$ e em $|M|$. Assim, os tamanhos das saídas das instâncias do problema \#CONCEPT não impedem a existência de um algoritmo de tempo polinomial para este problema. No entanto, a existência de um tal algoritmo é improvável: mostraremos em seguida o seguinte resultado, devido a Sergei Kuznetsov [Kuz01], que mostra que a contagem de conceitos é difícil.

Teorema 3.1. O problema \#CONCEPT é \#P-completo.

Prova. Será mostrada uma redução de tempo polinomial do problema \#2-SAT-MONótono ao \#CONCEPT. Seja $\left\{x_{1}, x_{2}, \ldots, x_{n}\right\}$ um conjunto de variáveis e seja $\phi$ uma fórmula do \#2-SAT-MONótono sobre as variáveis $\left\{x_{1}, x_{2}, \ldots, x_{n}\right\}$, isto é:

$$
\phi=\bigwedge_{i=1}^{s} C_{i}
$$

onde cada $C_{i}$ é uma cláulusa com dois literais positivos: $C_{i}=x_{i_{1}} \vee x_{i_{2}}$, com $i_{1}, i_{2} \in[n]$ para todo $1 \leq i \leq s$. Consideramos a negação de $\phi$, e a chamamos de $\psi$. Claramente, se soubermos o número de atribuições que satisfazem $\psi$, também saberemos em tempo polinomial o número de atribuições que satisfazem $\phi$, bastando para isso subtrair tal número de $2^{n}$. Para $i=1, \ldots, s$, seja $D_{i}=\overline{C_{i}}=\overline{x_{i_{1}}} \wedge \overline{x_{i_{2}}}$. Assim,

$$
\psi=\bar{\phi}=\bigvee_{i=1}^{s} \overline{C_{i}}=\bigvee_{i=1}^{s} D_{i}
$$

Seja $M=\left\{x_{1}, x_{2}, \ldots, x_{n}\right\}$ um conjunto, que será o conjunto de atributos de um contexto formal a ser construído. Desta maneira, os subconjuntos de $M$ estão em bijeção com o conjunto de atribuições de valores para $\left\{x_{1}, \ldots, x_{n}\right\}$. Mais especificamente, dado $S \subseteq M$, associamos a $S$ a atribuição que confere às variáveis de $S$ o valor "verdadeiro" e confere "falso" às demais.

Para cada conjunção $D_{i}$ de $\psi$, definiremos um contexto $\mathbb{K}_{i}=\left(G_{i}, M_{i}, I_{i}\right)$. O operador de derivação de objetos do contexto $\mathbb{K}_{i}$ será denotado por ${ }^{\triangleright_{i}}$, e seu operador de derivação

\footnotetext{
${ }^{4} \mathrm{Na}$ realidade, vale mais: $|\mathfrak{B}| \leq 2^{\min \{|G|,|M|\}}$, uma vez que a extensão de um conceito determina sua intenção e vice-versa. Veremos cotas superiores mais detalhadamente no Capítulo 7.
} 
de atributos por $\triangleleft_{i}$. Os conjuntos $G_{i}, M_{i}$ são definidos da seguinte maneira, assim como a relação $I_{i}$, que é definida através da derivação de conjuntos unitários de objetos:

$$
\begin{aligned}
G_{i} & =\left\{g_{0}^{i}, g_{1}^{i}, \ldots, g_{n-2}^{i}\right\} \\
M_{i} & =M \backslash\left\{x_{i_{1}}, x_{i_{2}}\right\}=\left\{m_{1}^{i}, m_{2}^{i}, \ldots, m_{n-2}^{i}\right\} \\
\left\{g_{0}^{i}\right\}^{\triangleright_{i}} & =M_{i} \\
\left\{g_{j}^{i}\right\}^{\triangleright_{i}} & =M_{i} \backslash\left\{m_{j}^{i}\right\}, \quad \text { para cada } j \in\{1,2, \ldots, n-2\} .
\end{aligned}
$$

Note que $G_{i}$ possui um elemento a mais que $M_{i}$, e que cada par de conjuntos $G_{i}, G_{j}$ com $i \neq j$ é disjunto. Vamos mostrar agora que o conjunto das intenções de cada $\mathbb{K}_{i}$ é o conjunto das partes $\mathcal{P}\left(M_{i}\right)$. Isto é:

$$
\operatorname{Int}\left(\mathbb{K}_{i}\right)=\mathcal{P}\left(M_{i}\right)
$$

Para mostrarmos (3.1), mostraremos que a função ${ }^{\triangleright_{i}}$ é sobrejetora, isto é, para todo $S \subseteq M_{i}$ existe $A \subseteq G_{i}$ tal que $A^{\triangleright_{i}}=S$. Note que isso, de fato, implica (3.1) pois, pela Proposição 2.3, item (iii), temos que $A^{\triangleright_{i} \triangleleft_{i} \triangleright_{i}}=A^{\triangleright_{i}}$. Assim, $\left(A^{\triangleright_{i} \triangleleft_{i}}, A^{\triangleright_{i}}\right)=\left(S^{\triangleleft_{i}}, S\right)$ é um conceito de $\mathbb{K}_{i}$. A fim de mostrarmos tal sobrejetividade, seja $S \subseteq M_{i}$ e $J$ o conjunto de índices dos elementos de $S$, isto é:

$$
J=\left\{j \in \mathbb{N} \mid m_{i}^{j} \in S, 1 \leq j \leq n-2\right\} .
$$

Agora, basta que tomemos $\bar{J}=\{1,2, \ldots, n-2\} \backslash J$ e $A=\left\{g_{i}^{j} \in G_{i} \mid j \in \bar{J}\right\}$, e é fácil verificar que $A^{\triangleright_{i}}=S$.

Definimos o contexto

$$
\mathbb{K}=\left(\bigcup_{i}^{s} G_{i}, M, \bigcup_{i}^{s} I_{i}\right)=(G, M, I)
$$

Denotaremos por ${ }^{\triangleright} \mathrm{e}^{\triangleleft}$ os operadores de derivação de $\mathbb{K}$. Agora, será mostrado que as intenções de $\mathbb{K}$ que são diferentes de $M$ estão em bijeção com as atribuições de variáveis que satisfazem a fórmula $\psi$, e isto concluirá a redução.

Seja, então, $B \in \operatorname{Int}(\mathbb{K}) \operatorname{com} B \neq M$. Vamos mostrar que $B \subseteq M_{i}$, para algum inteiro $i \in\{1,2, \ldots, s\}$. Suponha, por absurdo, que vale que $B \nsubseteq M_{i}$, para todo $i$. Portanto, para todo $i$, temos que $x_{i_{1}}$ ou $x_{i_{2}}$ pertence a $B$. Logo, para todo objeto $g_{j}^{i}$ do contexto $\mathbb{K}$, temos:

$$
g_{j}^{i} 干_{i} x_{i_{1}} \text { e } g_{j}^{i} 干_{i} x_{i_{2}} \Rightarrow g_{j}^{i} \notin B^{\triangleleft} \Rightarrow B^{\triangleleft}=\emptyset \Rightarrow B^{\triangleleft \triangleright}=M .
$$

Como $B \in \operatorname{Int}(\mathbb{K})$, temos pela Proposição 2.2 que $B=B^{\triangleleft \triangleright}=M$, o que contradiz a escolha de $B$. Portanto, vale que $B \subseteq M_{i}$ para algum $i$, e então $x_{i_{1}} \notin B$ e $x_{i_{2}} \notin B$. Consequentemente, a atribuição de valores para variáveis associada a $B$ atribui "falso" para $x_{i_{1}}$ e para $x_{i_{2}}$, e, consequentemente, satisfaz $D_{i}$, isto é, a $i$-ésima conjunção de $\psi$. Portanto, esta atribuição satisfaz $\psi$.

Com relação ao sentido contrário, considere agora uma atribuição de variáveis que satisfaça $\psi$. Seja $S$ o subconjunto de $M$ associado a tal atribuição. Mostraremos que $S \in \operatorname{Int}(\mathbb{K})$ e que $S \neq M$. Como a atribuição em questão satisfaz $\psi$, existe pelo menos uma conjunção $D_{i}$ de $\psi$ que é satisfeita por esta atribuição. Logo, $m_{i_{1}}, m_{i_{2}} \notin S$, e então

$$
S \subseteq M_{i}
$$


Observe que isto implica que $S \neq M$. De (3.1), temos que $S$ é intenção de $\mathbb{K}_{i}$. Seja $A$ a extensão de $\mathbb{K}_{i}$ associada a $S$, isto é, $A=S^{\triangleleft_{i}}$ e $A^{\triangleright_{i}}=S$. Observe que, como o objeto $g_{0}^{i}$ possui todos os atributos do contexto $\mathbb{K}_{i}$, segue que $A$ é não vazio. Agora, como $A^{\triangleright_{i}}=S$, temos que todo atributo de $S$ é apresentado por cada objeto de $A$, e ainda, cada atributo de $\bar{S}=M_{i} \backslash S$ não é apresentado por algum objeto de $A$. Ou seja:

$$
A^{\triangleright_{i}}=S \Leftrightarrow\left\{\begin{array}{l}
\left(\forall g_{j}^{i} \in A\right)(\forall x \in S) g_{j}^{i} I_{i} x \\
\left(\forall x \in M_{i} \backslash S\right)\left(\exists g_{j}^{i} \in A\right) g_{j}^{i} 干_{i} x .
\end{array}\right.
$$

Como $I_{i}$ é subconjunto de $G_{i} \times M_{i}$, temos que, para todo $x \in M \backslash M_{i}$ e todo $g_{j}^{i} \in G_{i}$, vale que $g_{j}^{i} I_{i} x$. Além disso, como $A$ é subconjunto não vazio de $G_{i}$, segue que:

$$
\left(\forall x \in M \backslash M_{i}\right)\left(\exists g_{j}^{i} \in A\right) g_{j}^{i} 干_{i} x .
$$

Utilizando o fato de que $S \subseteq M_{i} \subseteq M$, assim como (3.2) e (3.3), concluímos que:

$$
(\forall x \in M \backslash S)\left(\exists g_{j}^{i} \in A\right) g_{j}^{i} 干_{i} x .
$$

Como, para todo $k \in\{1, \ldots, s\}$ com $k \neq i$, a relação de incidência $I_{k}$ é um subconjunto de $G_{k} \times M_{k}$ e por construção vale que, para todo $j, g_{j}^{i} \notin G_{k}$, então temos que $g_{j}^{i} 干_{k} x$ para todo $j$, todo $k \neq i$ e todo $x \in M$. Juntando isto a (3.4), e usando o fato de que $I=\bigcup_{k=1}^{s} I_{k}$, temos que:

$$
(\forall x \in M \backslash S)\left(\exists g_{j}^{i} \in A\right) g_{j}^{i} \mp x .
$$

De (3.2), temos que, para todo $g_{j}^{i} \in A$ e todo $x \in S$ vale que $g_{j}^{i} I_{i} x$, e portanto, como $I_{i} \subseteq I$, claramente temos que:

$$
\left(\forall g_{j}^{i} \in A\right)(\forall x \in S) g_{j}^{i} I x
$$

Logo, os itens (3.5) e (3.6) equivalem a $A^{\triangleright}=S$, e portanto $S$ é intenção de $\mathbb{K}$. 



\section{Capítulo 4}

\section{Aplicações}

\subsection{Sistemas conceituais de informação}

Segundo Becker e Correia, Sistemas Conceituais de Informação (SCI) são "sistemas que armazenam, processam e apresentam informação usando representações orientadas a conceitos que amparam tarefas como: análise de dados, recuperação de informação e construção de teorias de maneira humanamente centrada" [BC04].

O ciclo de vida de um SCI é separado em duas fases. Primeiramente, ocorre a fase de criação de reticulados do sistema, quando cada reticulado de conceitos é determinado, bem como uma representação geométrica deste. Em um segundo momento, acontece a interação do usuário com os reticulados criados durante a primeira fase. Ambas as fases podem ser conduzidas, por exemplo, com o auxílio da suíte de software ToscanaJ [Tos11].

\subsubsection{Fase de criação de reticulados de um sistema}

Considere um conjunto de automóveis à venda. Cada automóvel possui propriedades, booleanas ou não, e que podem ser representadas em uma tabela, como em um banco de dados:

\begin{tabular}{|c|c|c|c|c|l|l|l|}
\hline código & válvulas & portas & combustível & loja & $\begin{array}{l}\text { vidro } \\
\text { elétrico }\end{array}$ & $\begin{array}{l}\text { trava } \\
\text { elétrica }\end{array}$ & $\begin{array}{l}\text { direção } \\
\text { hidráulica }\end{array}$ \\
\hline Gol 1.6 (1) & 8 & 2 & álcool & não & não & não & não \\
\hline Gol 1.0 (1) & 8 & 2 & gasolina & não & não & não & não \\
\hline Gol 1.0 (2) & 16 & 4 & gasolina & não & não & não & sim \\
\hline Gol 1.6 (2) & 8 & 4 & álc. e gasolina & sim & não & sim & sim \\
\hline$\vdots$ & & & & & & & \\
\hline
\end{tabular}

Tabela 4.1: Tabela de carros

Os dados presentes nas Tabelas 4.1 e 4.2 são um excerto de dados sobre 20 automóveis à venda em um sítio de classificados [Web11].

Na fase de criação de reticulados de um SCI, duas operações são tipicamente realizadas: as definições de escala e as projeções dos dados. A primeira operação é essencial para 


\begin{tabular}{|c|c|c|c|c|}
\hline ar condicionado & cilindrada & preço & kms rodados & modelo \\
\hline não & 1600 & $\mathrm{R} \$ 7.900$ & 121.000 & 1993 \\
\hline não & 1000 & $\mathrm{R} \$ 8.000$ & 81.846 & 1995 \\
\hline sim & 1000 & $\mathrm{R} \$ 14.990$ & 200.000 & 2002 \\
\hline não & 1600 & $\mathrm{R} \$ 22.000$ & 75.000 & 2004 \\
\hline$\vdots$ & & & & \\
\hline
\end{tabular}

Tabela 4.2: Tabela de carros (continuação)

tratar propriedades que não são booleanas, transformando-as em atributos no sentido de FCA. As projeções de dados servem para definir um aspecto do conjunto de dados e, como efeito colateral desejável, evitar que reticulados de conceitos fiquem demasiadamente grandes. Discorreremos sobre essas duas operações a seguir.

\section{Projeção dos dados}

A projeção de dados é uma operação muito simples: trata-se da escolha de um subconjunto dos atributos. Normalmente, tais subconjuntos possuem semântica precisa, como por exemplo, o conjunto dos opcionais de um carro. Podemos definir a projeção dos dados sobre carros, com relação aos opcionais, como sendo o seguinte conjunto de dados:

\begin{tabular}{|c|c|c|c|c|}
\hline código & vidro elétrico & trava elétrica & direção hidráulica & ar condicionado \\
\hline Gol $1.6(1)$ & não & não & não & não \\
\hline Gol $1.0(1)$ & não & não & não & não \\
\hline Gol $1.0(2)$ & não & não & sim & sim \\
\hline Gol $1.6(2)$ & não & sim & sim & não \\
\hline$\vdots$ & & & & \\
\hline
\end{tabular}

Tabela 4.3: Dados sobre opcionais dos carros.

A Tabela 4.3 torna-se o contexto formal da Tabela 4.4, bastando para tanto considerarmos que a propriedade "código" é simplesmente uma identificação de cada carro, o que, na linguagem de FCA, pode ser tratado como um objeto.

\begin{tabular}{|c|c|c|c|c|}
\hline & vidro elétrico & trava elétrica & direção hidráulica & ar condicionado \\
\hline Gol $1.6(1)$ & & & & \\
\hline Gol $1.0(1)$ & & & & \\
\hline Gol $1.0(2)$ & & & $\times$ & $\times$ \\
\hline Gol $1.6(2)$ & & & & \\
\hline$\vdots$ & & & & \\
\hline
\end{tabular}

Tabela 4.4: Contexto formal sobre opcionais dos carros.

O reticulado de conceitos do contexto formal da Tabela 4.4 está representado na Figura 4.1. Este e os demais reticulados deste capítulo foram criados com o auxílio do software 
ToscanaJ. Uma tarefa de investigação sobre o reticulado da Figura 4.1, bem como o detalhamento da rotulação ali utilizada serão feitos na Seção 4.1.2.

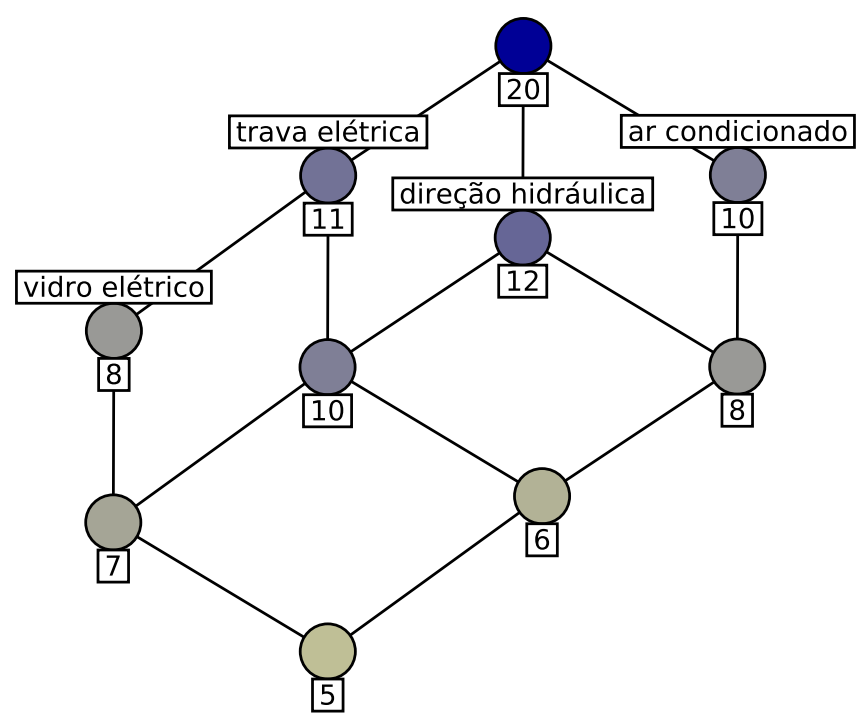

Figura 4.1: Reticulado de conceitos do contexto formal sobre opcionais.

\section{Definição de escala interordinal}

Nos SCIs, propriedades não booleanas frequentemente são propriedades que tomam valores no conjunto dos números reais. Para tratar tais propriedades, o usuário deve definir uma discretização do conjunto dos reais, utilizando predicados como a seguir. No exemplo, temos a propriedade "kms rodados". O usuário pode escolher como atributos " $<=130.000$ kms", "<=90.000 kms", "<=50.000 kms", ">130.000 kms", ">90.000 kms" e ">50.000 kms", resultando no seguinte contexto formal. Observe que omitimos os "kms" dos nomes dos atributos por questões de espaço.

\begin{tabular}{|c|c|c|c|c|c|c|}
\hline & $<130.000$ & $<90.000$ & $<50.000$ & $>=130.000$ & $>=90.000$ & $>=50.000$ \\
\hline Gol 1.6 (1) & $\times$ & & & & $\times$ & $\times$ \\
\hline Gol 1.0 (1) & $\times$ & $\times$ & & & & $\times$ \\
\hline Gol 1.0 (2) & & & & $\times$ & $\times$ & $\times$ \\
\hline Gol 1.6 (2) & $\times$ & $\times$ & & & & $\times$ \\
\hline$\vdots$ & & & & & & \\
\hline
\end{tabular}

Tabela 4.5: Contexto formal obtido após definição de escala interordinal.

O reticulado de conceitos do contexto formal da Tabela 4.5 está representado na Figura 4.2 .

O contexto da Tabela 4.5 poderia apresentar maior granularidade, isto é, poderíamos ter escolhido um número maior de atributos, de maneira a permitir uma análise mais refinada 


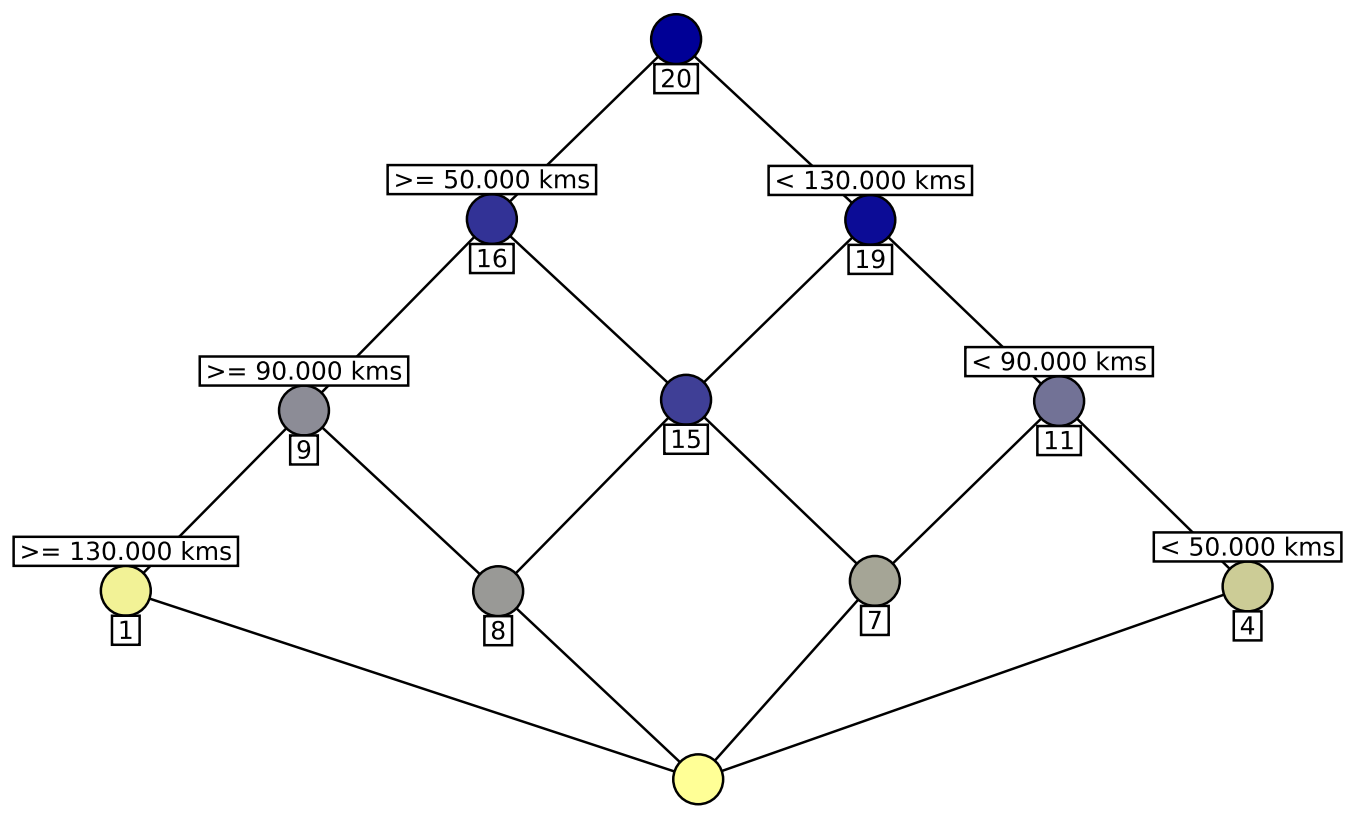

Figura 4.2: Reticulado de conceitos de uma escala interordinal, sobre kms rodados.

dos dados. Escolhemos esta quantidade de atributos (6) para facilitar uma representação explícita de seu contexto, em forma de tabela. Um reticulado sobre os preços dos carros, análogo ao da Figura 4.2, está representado na Figura 4.3.

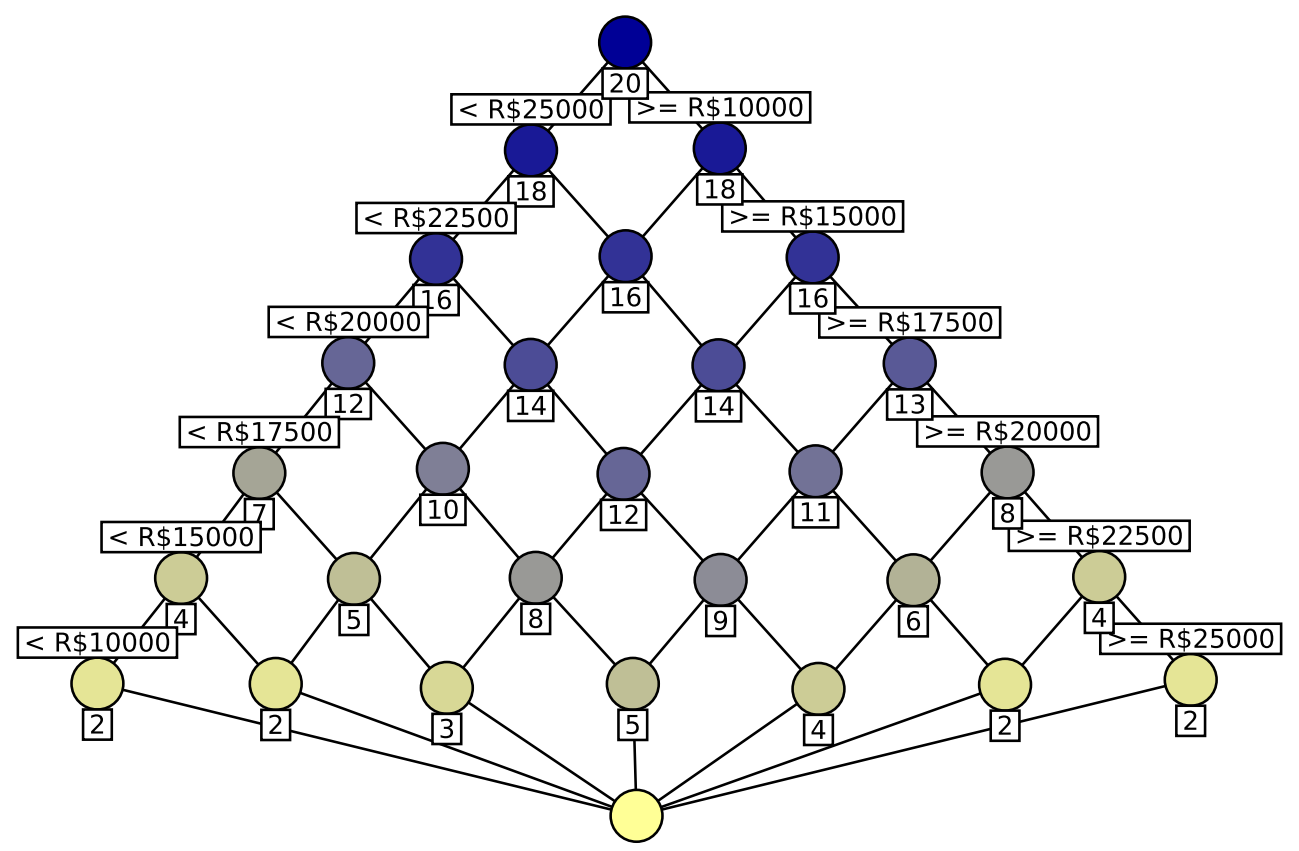

Figura 4.3: Reticulado de conceitos de uma escala interordinal, sobre preços. 


\section{Outras escalas}

Outra possibilidade para a ocorrência de propriedades não booleanas é a de propriedades enumerativas, como a propriedade "combustível", que assume três valores ("álcool", "gasolina" e "álcool e gasolina"). Para este tipo de propriedade, a criação de dois ou mais atributos é bastante natural, resultando em um contexto formal como o da Tabela 4.6.

\begin{tabular}{|c|c|c|}
\hline & a gasolina & a álcool \\
\hline Gol 1.6 (1) & & $\times$ \\
\hline Gol 1.0 (1) & $\times$ & \\
\hline Gol 1.0 (2) & $\times$ & \\
\hline Gol 1.6 (2) & $\times$ & $\times$ \\
\hline$\vdots$ & & \\
\hline
\end{tabular}

Tabela 4.6: Contexto formal sobre combustível dos carros.

\subsubsection{Fase de análise dos dados}

Durante a fase de análise de dados, os reticulados já foram criados e cabe ao usuário do sistema realizar tarefas de análise.

\section{Análise de reticulados individualmente}

Como foi argumentado, cada reticulado tipicamente reflete um aspecto do conjunto de dados. A análise de um destes reticulados já é suficiente para o usuário rapidamente adquirir informação sobre o conjunto de dados, mas sempre restrito ao aspecto associado ao reticulado em questão. Como exemplo, abaixo mostramos duas descobertas que um usuário pode rapidamente fazer, quando inspecionando o reticulado dos opcionais dos carros.

Na Figura 4.4, os rótulos acima de conceitos (rótulos de atributos) são como na Figura 2.6, quando definimos a rotulação reduzida. No entanto, os rótulos abaixo de conceitos estão representando as cardinalidades das extensões associadas aos respectivos conceitos. Esta rotulação permite ao usuário, para cada atributo $m$, tomar ciência da contagem de objetos que possuem o atributo $m$. Considere, por exemplo, o atributo $m=$ direção hidráulica. Da definição de rotulação reduzida, temos que $\mu(m)=\left(\{m\}^{\prime},\{m\}^{\prime \prime}\right)$, o que significa que a extensão do conceito em destaque na Figura 4.4(a) é o conjunto de automóveis que possuem o atributo $m$. Na rotulação aqui utilizada, não mostramos cada extensão, mas sim sua cardinalidade. Assim, vê-se na Figura 4.4(a) que há 12 carros com o atributo $m$, embora não saibamos quais são estes carros. Tomamos um segundo atributo, para fins de exemplificação: $n=$ ar condicionado. Desta maneira, a Figura 4.4(b) mostra que há 10 carros que possuem o atributo $n$. Naturalmente, podemos nos perguntar quantos carros possuem ambos os atributos $m$ e $n$. O Teorema 2.7 nos garante que existe o ínfimo dos conceitos $\mu(m)=\left(A_{1}, B_{1}\right)$ e $\mu(n)=\left(A_{2}, B_{2}\right)$, e que este ínfimo é exatamente o conceito

$$
\mu(m) \wedge \mu(n)=\left(A_{1} \cap A_{2},\left(B_{1} \cup B_{2}\right)^{\prime \prime}\right),
$$


o que significa que o conceito em foco na Figura 4.4(c) possui como extensão o conjunto dos carros que possuem ambos os atributos $m$ e $n$. Logo, há 8 carros com ambos os opcionais em questão. Note que a Figura 4.4(d) mostra que existem 5 carros com todos os opcionais.

Além da contagem de objetos que possuam um subconjunto de atributos, os reticulados de conceitos permitem a descoberta de implicações. Sejam, por exemplo, $m=$ trava elétrica e $n=$ vidro elétrico. Em qualquer reticulado da Figura 4.4, é fácil ver que o conceito $\mu(n)$ é menor ou igual ao conceito $\mu(m)$ (mais do que isto, vale que $\mu(n)$ é coberto por $\mu(m)$ ). Isto significa que a extensão de $\mu(n)$ está contida em $\mu(m)$, ou seja, $\{n\}^{\prime} \subseteq\{m\}^{\prime}$. Em outras palavras, neste conjunto de 20 carros, todo carro que possui vidro elétrico também possui trava elétrica.

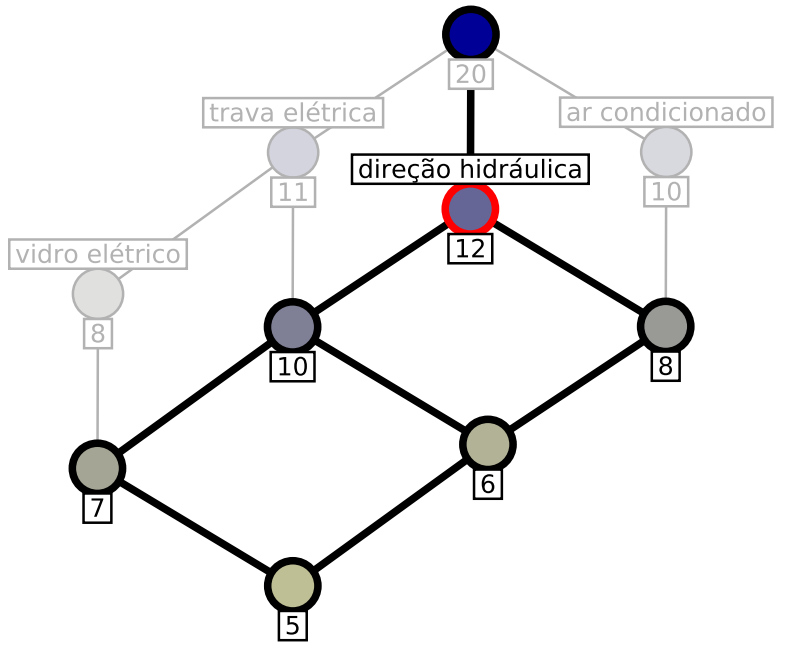

(a) Quantidade de carros com direção hidráulica.

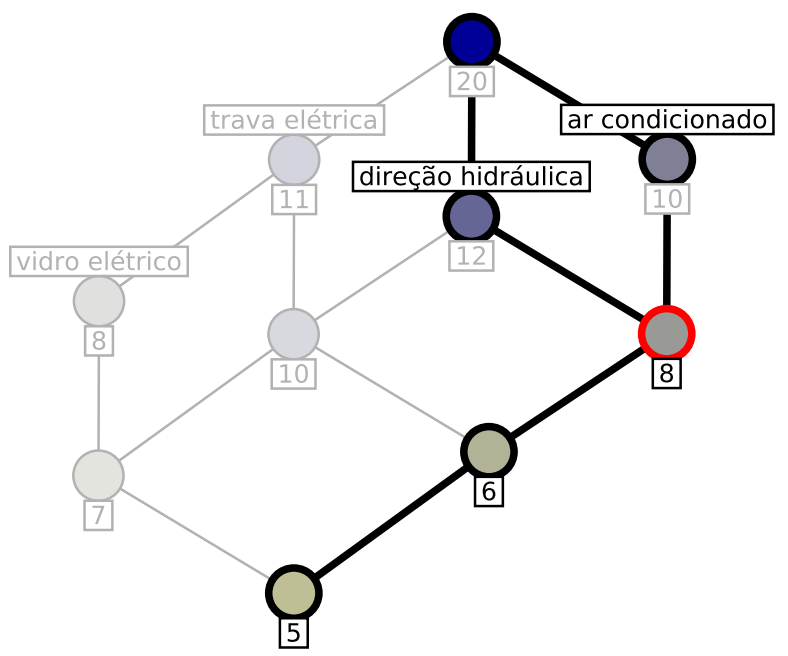

(c) Quantidade de carros com direção hidráulica e ar condicionado.

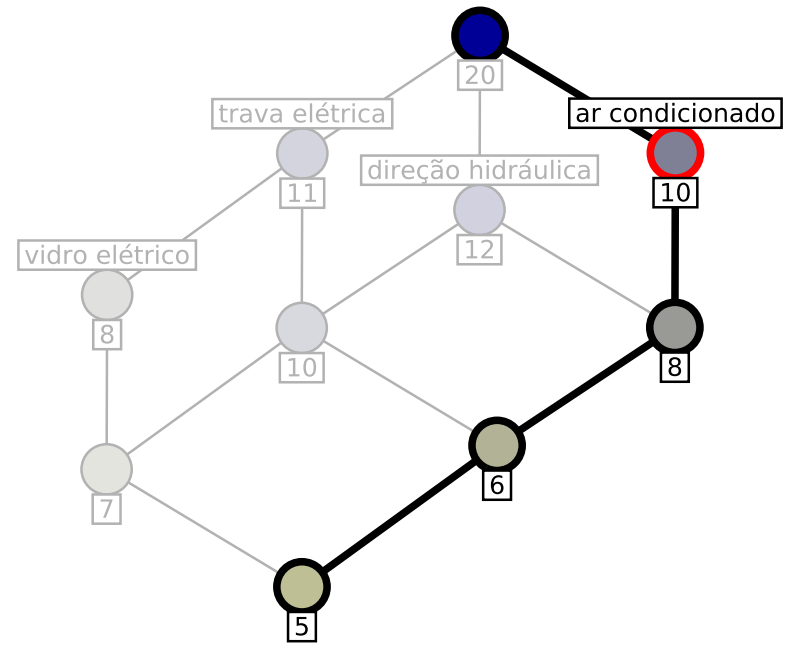

(b) Quantidade de carros com ar condicionado.

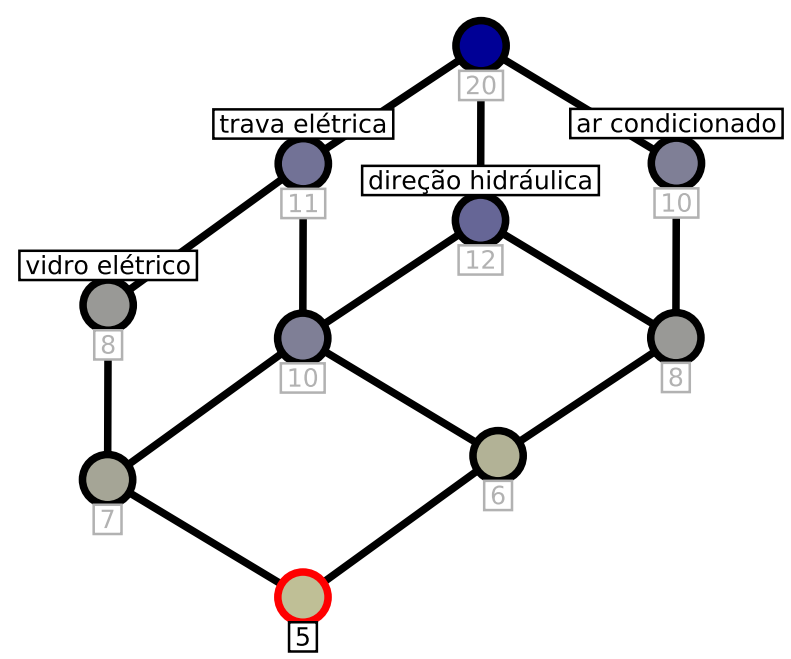

(d) Quantidade de carros com os quatro opcionais.

Figura 4.4: Análise do conjunto de carros sob o aspecto "opcionais". 
Reticulados de escalas interordinais possuem diagramas em forma de grade, que permitem facilmente a contagem de objetos que possuem a propriedade em questão pertencendo a cada intervalo da discretização. Um exemplo está presente na Figura 4.5.

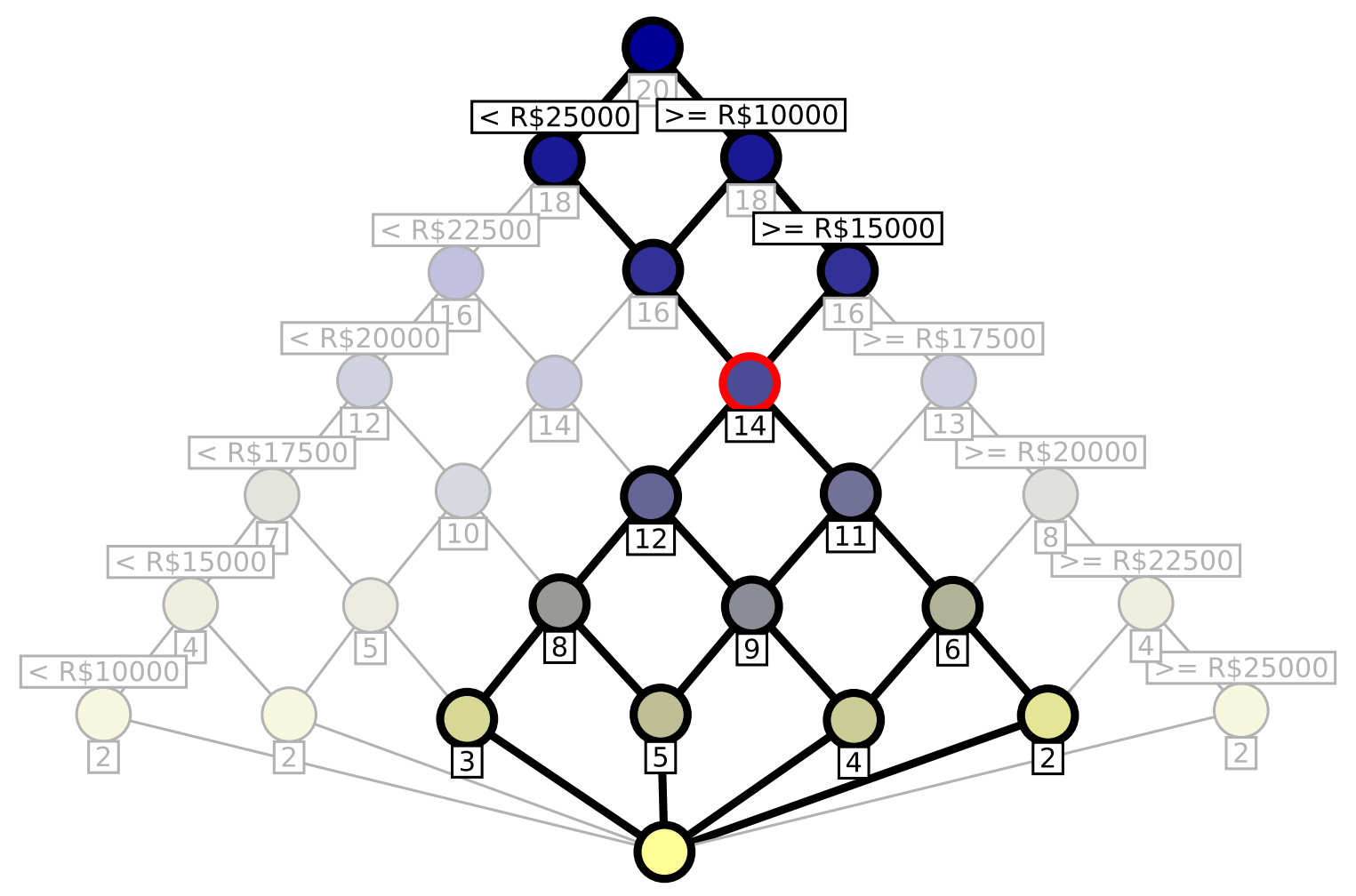

Figura 4.5: Reticulado sobre preços com foco no conceito de carros com preço entre $\mathrm{R} \$ 15.000$ e $R \$ 25.000$.

\section{Análise de vários reticulados: zoom}

A análise de um reticulado individualmente pode transmitir rapidamente informação sobre os dados a um usuário, mas de maneira restrita a um aspecto do conjunto de dados. É natural considerar um processo em que o usuário, durante a análise de um aspecto, elege um subconjunto de atributos, como por exemplo, \{direção $h$., ar condicionado $\}$, e deseja então analisar apenas objetos (carros) que possuam todos estes atributos, de acordo com um segundo aspecto (por exemplo, o preço de tais carros). A este processo se dá o nome de zoom.

A operação de zoom pode ser vista como a visualização de um reticulado de conceitos de um contexto $\left(G_{1}, M, I \cap\left(G_{1} \times M\right)\right)$ que é subcontexto de um contexto original $(G, M, I)$. É possível demonstrar que o reticulado de conceitos de um subcontexto é sempre uma subordem parcial do reticulado de conceitos do contexto original. Como a operação de zoom é realizada durante a fase de análise, e não durante a fase de construção dos reticulados, o software ToscanaJ exibe o reticulado do subcontexto em questão como uma sub-ordem parcial do reticulado original, aproveitando o diagrama já construido do reticulado original. 
O reticulado sobre preços, quando restrito aos carros que possuem direção hidráulica e ar condicionado, se torna o reticulado da Figura 4.6. Observe que, nesta figura, vários conceitos estão representados por círculos com diâmetro muito pequeno. Tais conceitos são chamados de conceitos não realizados, e são precisamente os conceitos do reticulado original que não pertencem ao reticulado do subcontexto.

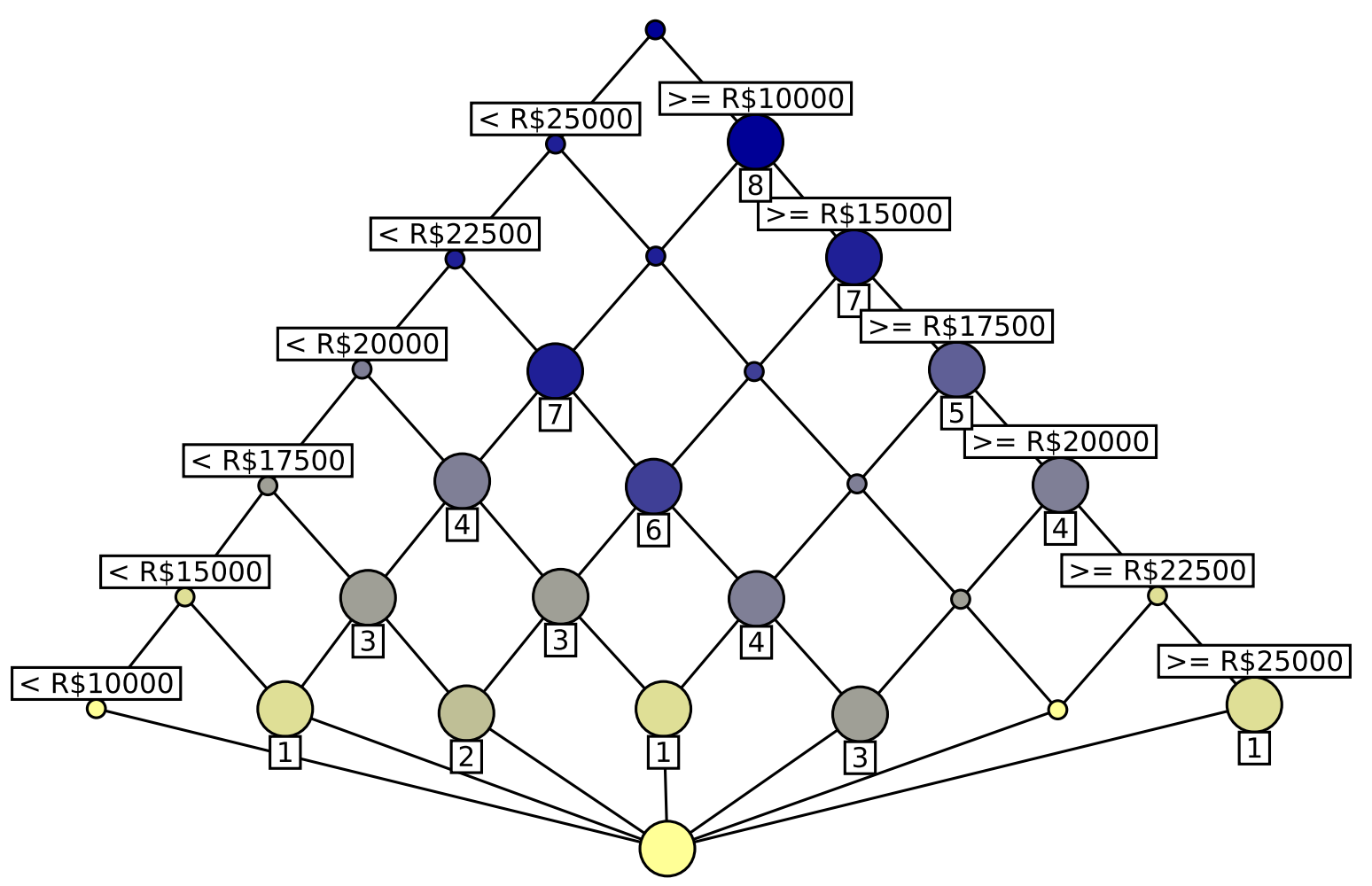

Figura 4.6: Reticulado sobre preços com zoom sobre carros com direção hidráulica e ar condicionado.

\subsubsection{Um exemplo de utilização}

Mostraremos um exemplo de uso de um SCI, utilizando como base o mesmo conjunto de dados sobre carros. Suponha que o usuário deseja tomar uma decisão sobre a compra de um carro, e inicialmente quer considerar apenas carros que custem menos que $\mathrm{R} \$ 22.500$. A análise por parte do usuário começa pelo reticulado representado na Figura 4.7.

Havendo 16 carros satisfazendo esta restrição sobre preços, o usuário deseja então analisar o aspecto "opcionais e motorização", e realiza zoom neste reticulado, obtendo o reticulado da Figura 4.8. Em tal reticulado, os atributos $>=1.3,>=1.6$ e $>=1.8$ se referem a cilindrada dos carros quando, respectivamente, são maiores ou iguais a 1300, 1600 e 1800.

Supondo que o usuário esteja interessado em carros com direção hidráulica, ar condicionado e que tenham 16 válvulas de motor, o usuário pode clicar no conceito associado a estes três atributos e verificar que há 4 carros satisfazendo todos os atributos considerados até agora. Como quatro carros constitui um conjunto relativamente pequeno de carros, mais 


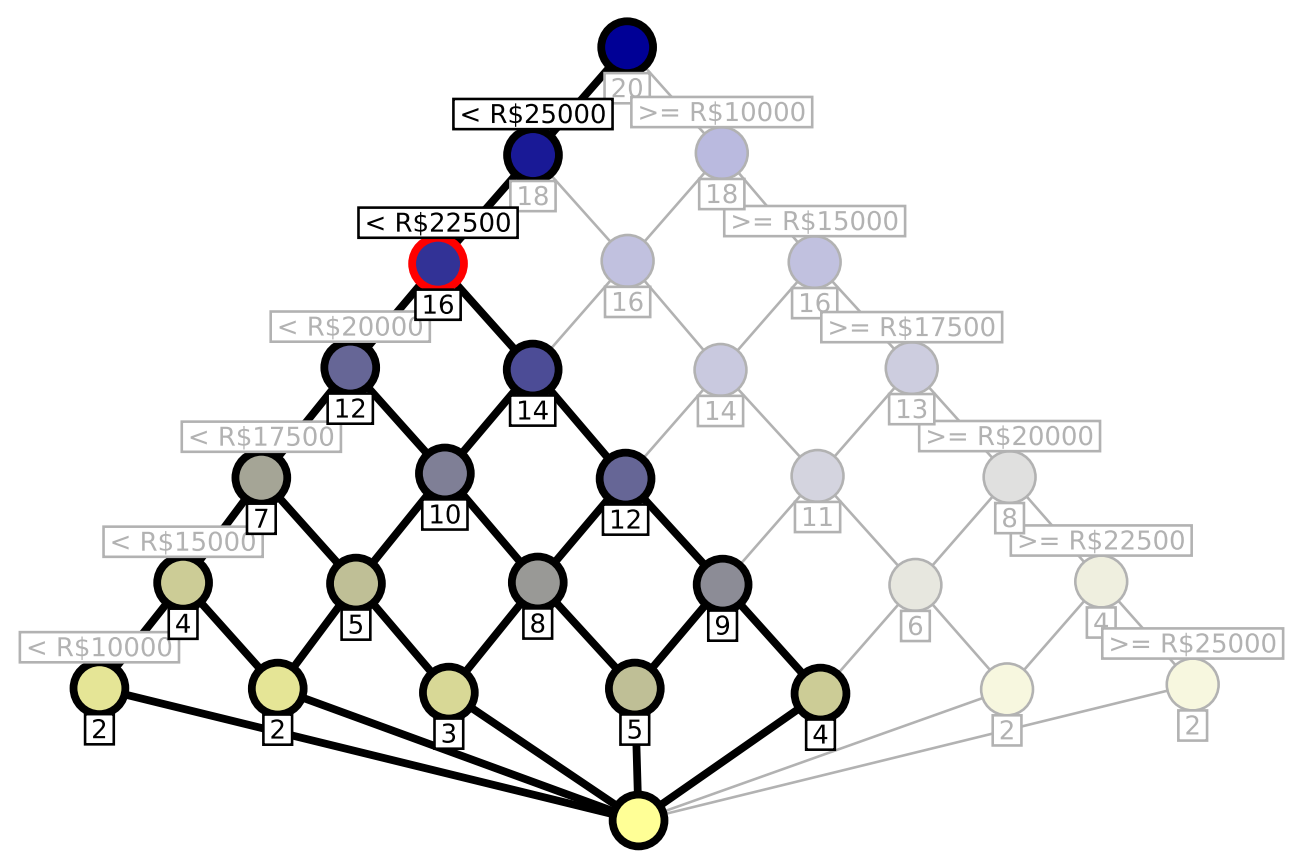

Figura 4.7: Reticulado sobre preços com foco em objetos (carros) com preço $<22500$.

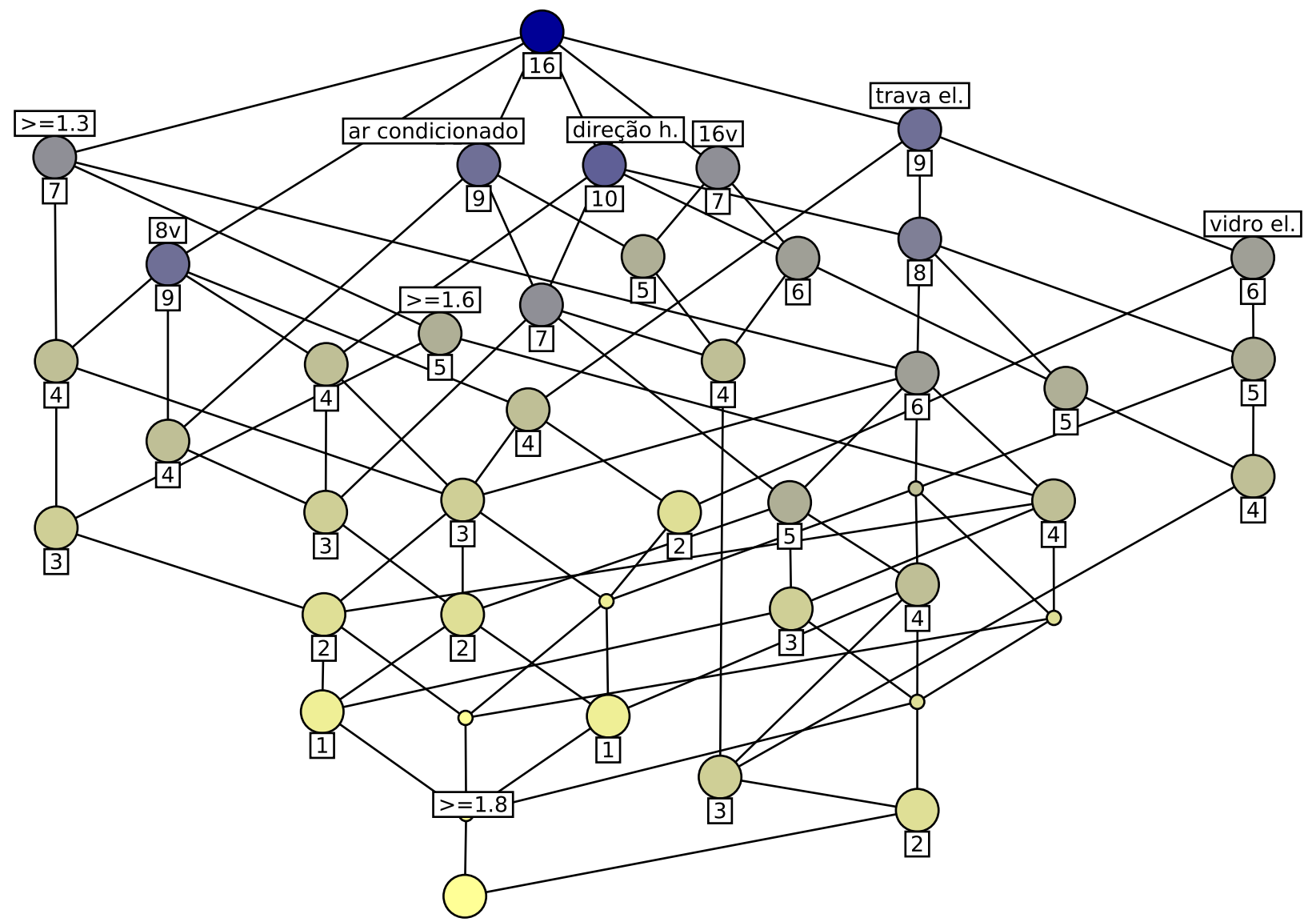

Figura 4.8: Reticulado sobre opcionais e motorização com zoom "preço < 22500". 
atenção pode ser dirigida a este conjunto por parte do usuário, que pode escolher visualizar o código dos quatro carros, como na Figura 4.9.

Após a análise do reticulado das Figuras 4.8 e 4.9, o usuário pode, se quiser, retroceder o processo de zoom que foi realizado, efetuando o que é chamado de drill up ${ }^{1}$. Neste exemplo, após um drill up, o usuário poderia então analisar carros cujo preço são iguais ou superiores a $\mathrm{R} \$ 22.500$. Alternativamente, o usuário pode manter o zoom atual e realizar um segundo zoom, como por exemplo sobre o aspecto "vendedor", para saber se quem está vendendo tais quatro carros são lojas ou pessoas físicas ("particulares"). Neste caso, um reticulado como o da Figura 4.10 seria apresentado para o usuário.

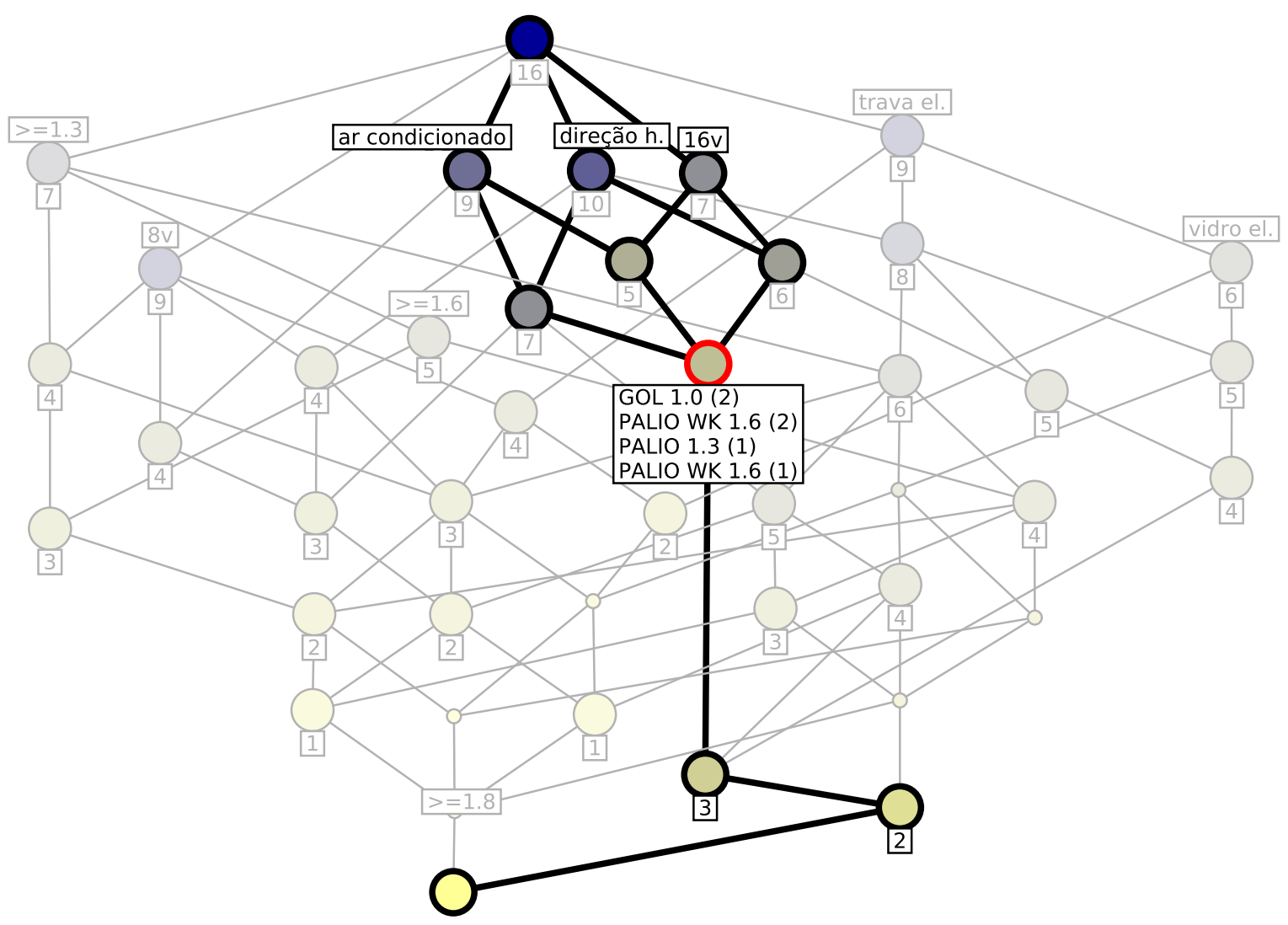

Figura 4.9: Reticulado sobre opcionais e motorização com zoom "preço < 22500" e foco.

Ao visualizar o reticulado da Figura 4.10, o usuário pode escolher visualizar mais informação sobre, por exemplo, os três carros que são vendidos por particulares. Esta visualização de informação pode simplesmente ser a exibição da tabela de dados, como nas Tabelas 4.1 e 4.2, ou senão, a exibição de um relatório, que pode incluir as fotos dos carros em questão, como na Figura 4.11.

\footnotetext{
${ }^{1} \mathrm{O}$ que chamamos de zoom também é conhecido na literatura como drill down, por isso seu antônimo ser drill up.
} 


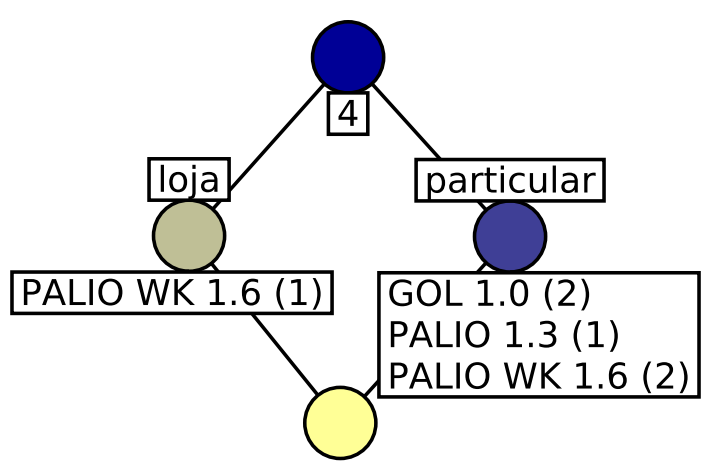

Figura 4.10: Reticulado sobre realizadores de venda dos carros, após dois zooms.

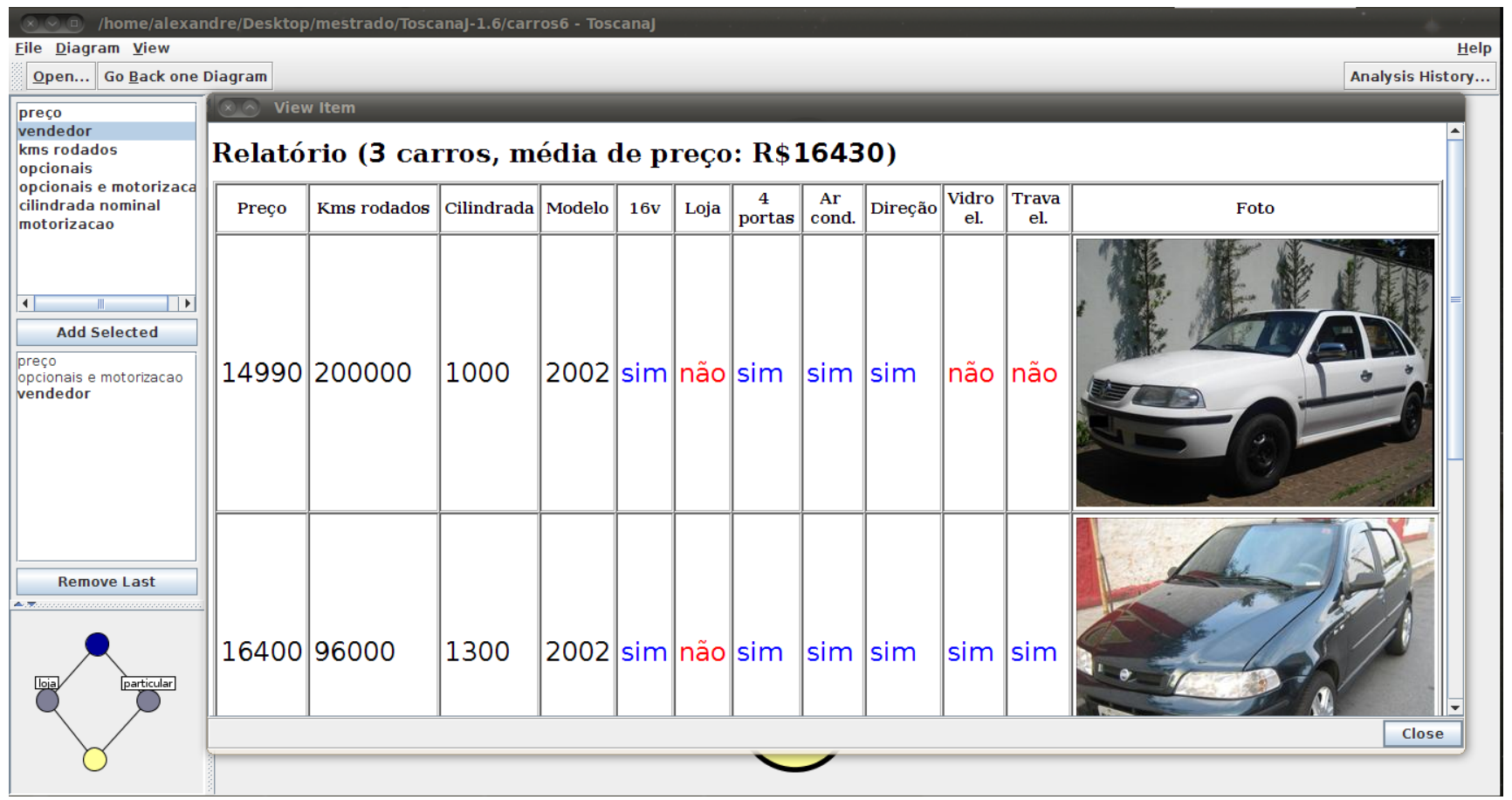

Figura 4.11: Relatório exibindo três carros.

\subsubsection{Sistemas Conceituais de Informação implantados}

Becker e Correia relatam em 2004 [BC04] que, desde o início dos anos 1990, muitos Sistemas Conceituais de Informação foram desenvolvidos e implantados com finalidades diversas, tais como:

- Análise de dados médicos sobre crianças com diabetes $\left[\mathrm{SSV}^{+} 93\right]$

- Exploração de leis e regulações em engenharia civil [KSVW94]

- Busca de livros em uma biblioteca [SKSW95]

- Auxílio para engenheiros na elaboração de encanamentos [Vog95] 
- Análise de paradigmas de verbos em lingüística [Gro96]

- Refatoração de código e design de hierarquia de classes em engenharia de software [DH98], [LS97]

- Investigação de cooperação internacional em ciências políticas [KKV00]

- Processamento analítico online (OLAP) e descoberta de conhecimento em banco de dados (Knowledge Discovery in Databases), na área de data warehousing [HSWW00], [CSWW03], [Stu00]

Mais recentemente, Jonas Poelmans afirma em [Poe10] que, de 2003 até 2009, foram publicados mais de 700 artigos nos quais FCA era utilizada de alguma maneira. Em 2009, o trabalho [PEVD09] recebeu o prêmio de melhor artigo de jovens profissionais (Young professionals best paper award) na International Conference on Data Mining, em Leipzig. Neste trabalho, Poelmans e outros utilizaram de maneira central as técnicas de Formal Concept Analysis para analisar informações, previamente extraídas e tratadas, de relatórios sobre violência doméstica da polícia da cidade de Amsterdã. Através deste processo de análise, foi possível compreender e mitigar ineficiências nos processos realizados pelo departamento de polícia, desta maneira melhorando o atendimento à população. Após a conclusão deste trabalho, o mesmo departamento de polícia requisitou um projeto similar mas, desta vez, para dados sobre tráfico humano [Poe10]. Três dos autores de [PEVD09] trabalharam em um projeto para melhoramento de processos hospitalares (integrated care pathways) de um hospital belga, novamente empregando técnicas de FCA. Seus resultados publicados em $\left[\mathrm{PDV}^{+} 10\right]$ resultaram no prêmio de melhor artigo da International Conference on Data Mining, em 2010, na cidade de Berlim.

\subsection{FCA em recuperação de informação}

Uma biblioteca digital é um sistema de catálogo de documentos munido de uma interface de consultas voltada para o usuário. Com o advento da web 2.0, tornou-se cada vez mais comum o etiquetamento de cada documento da biblioteca com metadados: isto é, dados que descrevem propriedades de cada documento, seja sobre o assunto tratado no documento, os autores, sua data de criação, seja sobre outras características do documento. Tal etiquetamento visa estabelecer uma emissão rápida de informações sobre um documento para o usuário.

Consideramos agora uma biblioteca na qual cada documento está etiquetado com palavraschave correspondentes aos assuntos tratados por aquele documento. Assim, identificamos cada palavra-chave com o que chamaremos de assunto. Um exemplo de resposta a uma consulta, pela expressão "polynomial delay" está presente na Figura 4.12.

Na Figura 4.12, vê-se que a resposta do sistema com relação à consulta realizada pelo usuário se apresenta exclusivamente em forma de tabela. Como de costume, os documentos presentes na tabela apresentam, de cima para baixo, ordem decrescente de relevância, segundo alguma métrica do sistema [MRS08].

Segundo Carpineto e Romano, resultados de buscas em bibliotecas digitais quase que invariavelmente se apresentam ao usuário de maneira tabular, como retratado na Figura 4.12. 


\begin{tabular}{l} 
Busca \\
\begin{tabular}{|l|l|}
\hline Resulynomial delay" \\
\hline An old solution of the maximal biclique generation problem \\
Palavras-chave: Formal Concept Analysis, Bicliques maximais, Grafos: teoria e desenhos, Algoritmos, Teoria da Computacao \\
A linear delay algorithm for building concept lattices - presentation \\
Palavras-chave: Construcao de Reticulado, Formal Concept Analysis, Algoritmos, Teoria da Computacao \\
Comparing Performance of Algorithms for Generating Concept Lattices \\
Palavras-chave: Construcao de Reticulado, Formal Concept Analysis, Algoritmos, Teoria da Computacao \\
Algorithms for the Construction of Concept Lattices and Their Diagram Graphs \\
Palavras-chave: Construcao de Reticulado, Formal Concept Analysis, Algoritmos, Teoria da Computacao \\
A linear delay algorithm for building concept lattices \\
Palavras-chave: Construcao de Reticulado, Formal Concept Analysis, Algoritmos, Teoria da Computacao \\
On Independent Sets and Bicliques in Graphs \\
Palavras-chave: Bicliques maximais, Conjuntos independentes, Grafos: teoria e desenhos \\
Faster Algorithms for Constructing a Concept (Galois) Lattice \\
Palavras-chave: Construcao de Reticulado, Formal Concept Analysis, Algoritmos, Teoria da Computacao \\
On the Intractability of Computing the Duquenne-Guigues Base \\
Palavras-chave: Formal Concept Analysis, Regras de Associacao, Inteligencia Artificial, Complexidade Computacional, Teoria \\
da Computacao \\
Consensus algorithms for the generation of all maximal bicliques \\
Palavras-chave: Bicliques maximais, Grafos: teoria e desenhos, Algoritmos, Teoria da Computacao \\
Some open problems in Formal Concept Analysis \\
Palavras-chave: Formal Concept Analysis
\end{tabular} \\
\hline
\end{tabular}

Figura 4.12: Interface convencional de sistema de recuperação de informação.

Além disso, eles argumentam em [CR04] que tal forma de transmissão de informação dificulta a obtenção de respostas a certas perguntas sobre a coleção retornada, tais como:

1. Existe algum assunto ubíquo (isto é, tratado por todos os documentos)?

2. Além dos ubíquos, quais são os assuntos "mais gerais" tratados pelos documentos?

3. Dado um assunto $t$, quantos (e quais) documentos tratam do assunto $t$ ?

4. Dados assuntos $t_{1}$ e $t_{2}$, quantos (e quais) documentos tratam dos assuntos $t_{1}$ e $t_{2}$ ? Mais geralmente, dados assuntos $t_{1}, t_{2}, \ldots, t_{k}$ quantos (e quais) documentos tratam de cada assunto $t_{i}, \operatorname{com} i=1, \ldots, k$ ?

Em [CR04], os autores argumentam que uma interface que empregue alguma representação de reticulado de conceitos mitiga tais dificuldades presentes em interfaces tradicionais. Um exemplo de interface com uma representação geométrica explícita de um reticulado de conceitos está presente na Figura 4.13. O contexto formal associado ao reticulado de tal figura é dado pela incidência das palavras-chaves (atributos) nos documentos (objetos).

Utilizando-se um reticulado de conceitos, a questão da existência de assunto ubíquo é facilmente endereçada, bastando para o usuário verificar se o elemento máximo do reticulado recebeu algum rótulo superior, conforme vimos anteriormente.

Com relação à questão número 2, Carpineto e Romano argumentam que o conjunto de rótulos superiores dos coátomos representam os assuntos não ubíquos mais gerais tratados pela coleção retornada. No exemplo da Figura 4.13, tais assuntos são "Formal Concept Analysis", "Teoria da Computação", "Bicliques maximais" e "Grafos: teoria e desenhos". 


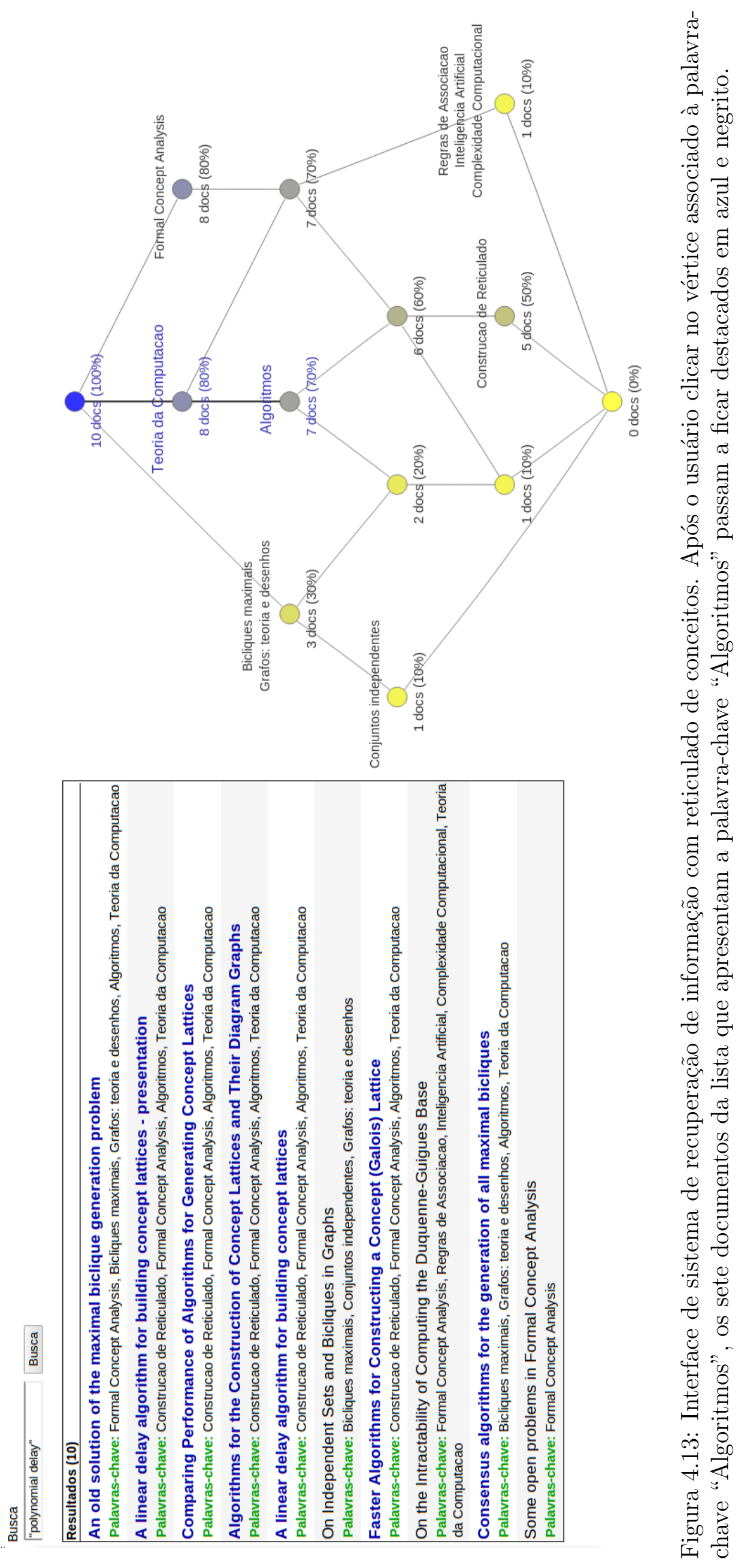


A representação do diagrama do reticulado permite que o usuário visualize, de maneira global, seus rótulos superiores. Com isso, o usuário toma conhecimento de quais palavraschave estão presentes na coleção retornada. Se alguma das palavras-chave, digamos, $t$, desperta o interesse do usuário, ele pode verificar a contagem de quantos documentos estão etiquetados com $t$, bastando para isto verificar o rótulo inferior associado a tal vértice. Se, além disso, o usuário quiser saber quais são tais documentos, basta ao usuário clicar no vértice, obtendo um resultado como o retratado na Figura 4.13, onde supomos que o usuário se interessou por $t=$ Algoritmos. Naquela figura, os títulos dos sete documentos que apresentam tal palavra-chave estão em azul e negrito. Naturalmente, o usuário pode se interessar por duas palavras-chave, digamos, $t_{1}$ e $t_{2}$ e tomar o ínfimo $\mu\left(t_{1}\right) \wedge \mu\left(t_{2}\right)$, a fim de obter a informação de quais documentos apresentam ambas as palavras-chave $t_{1}$ e $t_{2}$. A operação de ínfimo pode ser realizado com um número arbitrário de operandos, respondendo as questões 3 e 4 que foram enumeradas. 



\section{Capítulo 5}

\section{Determinação do conjunto de conceitos}

Neste capítulo, consideramos o problema de se encontrar o conjunto de conceitos de um contexto finito. Apresentaremos dois algoritmos para este problema. O primeiro algoritmo que será exposto segue a partir de uma caracterização das intenções, e de uma recursão que permite o cálculo eficiente dos subconjuntos presentes nesta caracterização. Veremos que este algoritmo apresenta complexidade de tempo $O(|\mathfrak{B}| \cdot|G| \cdot|M|)$.

O segundo algoritmo que será mostrado é um algoritmo de atraso polinomial: a quantidade de tempo gasta entre quaisquer impressões ${ }^{1}$ de conceitos, assim como a quantidade de tempo dispendiada antes da primeira impressão, bem como entre a última impressão de conceito e o encerramento do algoritmo, são polinomiais em $|G|$ e $|M|$. Mais especificamente, mostraremos que este algoritmo possui atraso $O\left(|G| \cdot|M|^{2}\right)$, o que confere a esta solução uma complexidade de tempo total $O\left(|\mathfrak{B}| \cdot|G| \cdot|M|^{2}\right)$.

Estes algoritmos encontram os conceitos, sem contudo encontrar os arcos do diagrama do reticulado, o que é feito pelos algoritmos do Capítulo 6.

\subsection{Fecho por intersecções de intenções-objetos}

Seja $\mathbb{K}=(G, M, I)$ um contexto. Assim como na demonstração do Teorema 2.8, escreveremos $g^{\prime}$ em vez do correto $\{g\}^{\prime}$. Para todo $g \in G$, chamamos um conjunto $g^{\prime} \subseteq M$ de intenção-objeto. Este nome justifica-se, já que $\left(g^{\prime \prime}, g^{\prime}\right)$ é sempre um conceito, para qualquer $g \in G$, e portanto $g^{\prime}$ é sempre uma intenção.

Visando um algoritmo que encontra os conceitos de um contexto, focaremos primeiramente em um algoritmo que encontra suas intenções. Antes disso, faremos uma definição: dado um conjunto $X$ qualquer, uma família de subconjuntos de $X$ (ou simplesmente, família) é um subconjunto $\mathcal{F} \subseteq \mathcal{P}(X)$. Em contraste com as famílias indexadas, estas famílias não estão associadas a um conjunto de índices. Esta definição será amplamente usada durante este e o próximo capítulos.

Na Proposição 5.1, mostramos uma caracterização das intenções de $\mathbb{K}$, em função das intersecções de intenções-objetos.

\footnotetext{
${ }^{1}$ Impressão, neste contexto, significa "devolução na saída".
} 
Proposição 5.1. Seja $\mathbb{K}$ um contexto. O conjunto de intenções de $\mathbb{K}$ pode ser descrito como

$$
\operatorname{Int}(\mathbb{K})=\left\{\bigcap_{g \in S} g^{\prime} \mid S \subseteq G\right\}
$$

Prova. Seja $B$ uma intenção de $\mathbb{K}$ e $A$ sua extensão associada, isto é, $A=B^{\prime}$ e $A^{\prime}=B$. Temos que

$$
B=\left(\bigcup_{g \in A}\{g\}\right)^{\prime}
$$

Logo, pelo Lema 2.6, segue que

$$
B=\bigcap_{g \in A} g^{\prime}
$$

Concluímos que toda intenção é intersecção de um conjunto de intenções-objetos, isto é, toda intenção é da forma $\bigcap_{g \in S} g^{\prime}$, para algum $S \subseteq G$. Provaremos agora que a recíproca é verdadeira, ou seja, todo elemento da família de subconjuntos $\mathcal{F} \subseteq \mathcal{P}(M)$, onde

$$
\mathcal{F}=\left\{\bigcap_{g \in S} g^{\prime} \mid S \subseteq G\right\}
$$

é uma intenção. De fato, se $B$ for um subconjunto em $\mathcal{F}$, então temos que $B=\bigcap_{g \in S} g^{\prime}$ para algum $S \subseteq G$ e vale o seguinte.

$$
\begin{aligned}
\left(B^{\prime}, B\right) & =\left(\left(\bigcap_{g \in S} g^{\prime}\right)^{\prime}, \bigcap_{g \in S} g^{\prime}\right) \\
& =\left(\left(\bigcup_{g \in S}\{g\}\right)^{\prime \prime},\left(\bigcup_{g \in S}\{g\}\right)^{\prime}\right) \quad \text { (pelo Lema 2.6). }
\end{aligned}
$$

Ou seja, $\left(B^{\prime}, B\right)$ é da forma $\left(A^{\prime \prime}, A^{\prime}\right)$. Pela propriedade iii) da Proposição 2.3 , temos que todo par da forma $\left(A^{\prime \prime}, A^{\prime}\right) \operatorname{com} A \subseteq G$ é um conceito. Consequentemente, $\left(B^{\prime}, B\right)$ é um conceito, e portanto $B$ é uma intenção de $\mathbb{K}$.

A Proposição 5.1 já inspira um algoritmo ingênuo para se encontrar o conjunto das intenções: o cálculo de $\bigcap_{g \in S} g^{\prime}$, para cada $S \subseteq G$. No entanto, esse algoritmo possui a desvantagem de apresentar complexidade computacional $\Omega\left(2^{|G|}\right)$, independentemente da cardinalidade de $\operatorname{Int}(\mathbb{K})$. Esta desvantagem contrasta com o objetivo aqui buscado, que é apresentar um algoritmo sensível à saída, e que possa ter seu tempo de execução delimitado superiormente por um polinômio em $|\operatorname{Int}(\mathbb{K})|,|G|$ e $|M|$. Ainda assim, a caracterização da Proposição 5.1 nos será útil e, para isso, consideramos adicionalmente que o conjunto $G$ se encontra sob alguma ordenação: $G=\left\{g_{i}|i=1, \ldots| G \mid,\right\}$. É claro que, com esta ordenação, temos uma equação análoga à contida na Proposição 5.1:

$$
\operatorname{Int}(\mathbb{K})=\left\{\bigcap_{i \in S} g_{i}^{\prime} \mid S \subseteq\{1, \ldots,|G|\}\right\} .
$$


Ainda considerando $G$ ordenado, vamos mostrar uma recorrência que permite um cálculo eficiente de $\operatorname{Int}(\mathbb{K})$. Definimos:

$$
\mathcal{L}_{i}= \begin{cases}\{M\}, & \text { se } i=0 \\ \mathcal{L}_{i-1} \cup\left\{g_{i}^{\prime} \cap B \mid B \in \mathcal{L}_{i-1}\right\}, & \text { se } 1 \leq i \leq|G|\end{cases}
$$

A próxima proposição mostra que a recorrência $\mathcal{L}_{i}$ pode ser utilizada para obtermos o conjunto $\operatorname{Int}(\mathbb{K})$.

Proposição 5.2. Sejam $\mathbb{K}=(G, M, I)$ um contexto e $\left\{g_{i}|i=1, \ldots| G \mid,\right\}$ uma ordenação dos elementos de G. Então,

$$
\mathcal{L}_{|G|}=\left\{\bigcap_{i \in S} g_{i}^{\prime} \mid S \subseteq\{1, \ldots,|G|\}\right\}
$$

e, consequentemente, $\mathcal{L}_{|G|}=\operatorname{Int}(\mathbb{K})$.

Prova. Procederemos por indução em $|G|$. Se $|G|=0$, então $L_{|G|}=\{M\}$, por definição, e claramente $\left\{\bigcap_{i \in S} g_{i}^{\prime} \mid S \subseteq\{1, \ldots,|G|\}\right\}=\{M\}$. Suponha que $|G|=n$ e que a hipótese de indução vale para $n-1$.

Provaremos que

$$
\mathcal{L}_{n} \subseteq\left\{\bigcap_{i \in S} g_{i}^{\prime} \mid S \subseteq\{1, \ldots, n\}\right\} .
$$

Seja $B \in \mathcal{L}_{n}$. Suponha o caso em que $B \in \mathcal{L}_{n-1}$. Então, pela hipótese de indução, temos que

$$
B \in\left\{\bigcap_{i \in S} g_{i}^{\prime} \mid S \subseteq\{1, \ldots, n-1\}\right\} \Rightarrow B \in\left\{\bigcap_{i \in S} g_{i}^{\prime} \mid S \subseteq\{1, \ldots, n\}\right\} .
$$

Agora, suponha o caso em que $B \notin \mathcal{L}_{n-1}$. Logo, pela definição da recorrência $\mathcal{L}$, temos que existe $Y \in \mathcal{L}_{n-1}$ tal que $B=g_{n}^{\prime} \cap Y$. Como $Y \in \mathcal{L}_{n-1}$, pela hipótese de indução segue que $Y \in\left\{\bigcap_{i \in S} g_{i}^{\prime} \mid S \subseteq\{1, \ldots, n-1\}\right\}$. Assim,

$$
\left.\begin{array}{l}
Y \in\left\{\bigcap_{i \in S} g_{i}^{\prime} \mid S \subseteq\{1, \ldots, n-1\}\right\} \\
B=g_{n}^{\prime} \cap Y
\end{array}\right\} \Rightarrow B \in\left\{\bigcap_{i \in S} g_{i}^{\prime} \mid S \subseteq\{1, \ldots, n\}\right\} .
$$

Resta provar que

$$
\left\{\bigcap_{i \in S} g_{i}^{\prime} \mid S \subseteq\{1, \ldots, n\}\right\} \subseteq \mathcal{L}_{n}
$$

Seja $S \subseteq\{1, \ldots, n\}$ e considere o conjunto $\bigcap_{i \in S} g_{i}^{\prime}$. Suponha o caso em que $n \notin S$. Assim,

$$
\bigcap_{i \in S} g_{i}^{\prime} \in\left\{\bigcap_{i \in S} g_{i}^{\prime} \mid S \subseteq\{1, \ldots, n-1\}\right\},
$$

e, pela hipótese de indução, vale que $\cap_{i \in S} g_{i}^{\prime} \in \mathcal{L}_{n-1} \subseteq \mathcal{L}_{n}$.

Suponha o caso em que $n \in S$. Então, segue que $\bigcap_{i \in S} g_{i}^{\prime}$ é da forma $g_{n}^{\prime} \cap B$, com $B \in\left\{\bigcap_{i \in S} g_{i}^{\prime} \mid S \subseteq\{1, \ldots, n-1\}\right\}$. Novamente pela hipótese de indução, $B \in \mathcal{L}_{n-1}$, portanto $\bigcap_{i \in S} g_{i}^{\prime} \in \mathcal{L}_{n}$, pela definição da recorrência $\mathcal{L}_{i}$. 
O Algoritmo 1 recebe um contexto $\mathbb{K}=(G, M, I)$ e encontra o conjunto $\mathcal{L}_{|G|}=\operatorname{Int}(\mathbb{K})$. É fácil verificar esta afirmação, já que, imediatamente após a $i$-ésima iteração do laço da linha 2, temos que o valor de $\mathcal{F}$ é $\mathcal{L}_{i}$. Para encontrarmos o conjunto de todos os conceitos, podemos realizar um pós processamento sobre $\mathcal{F}$ que faz o cálculo da derivação, $B^{\prime}$, de cada intenção $B \in \mathcal{F}$.

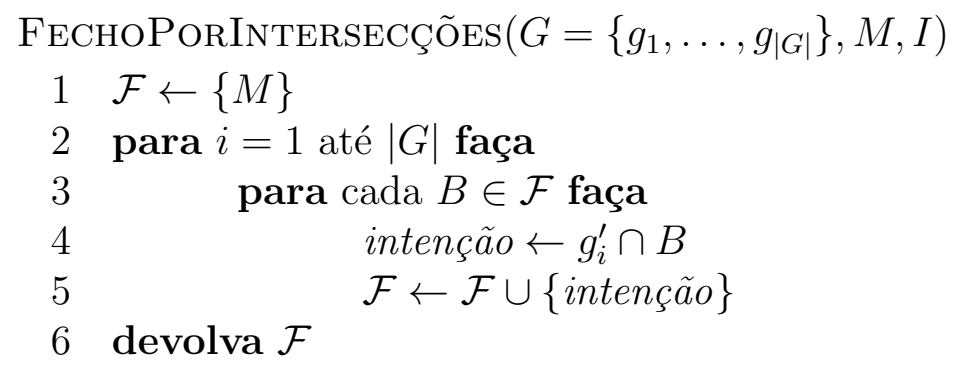

Algoritmo 1: Algoritmo para determinação das intenções.

\section{Um exemplo de execução}

Daremos a seguir um exemplo de execução do Algoritmo 1. Antes, explicaremos como serão representados os conjuntos durante a simulação. Seja $X=\left\{x_{i}|i=1, \ldots| X \mid,\right\}$ um conjunto ordenado. A ordenação de $X$ nos permite representar subconjuntos $A \subseteq X$ por palavras de comprimento $|X|$ sobre o alfabeto $\{0,1\}$. Mais especificamente, para cada subconjunto $A$ associamos a palavra $p=p_{1} p_{2} \ldots p_{|X|}$, onde $p_{i}=1$ se $p_{i} \in A$ e $p_{i}=0$ se $p_{i} \notin A$, para $i=1, \ldots,|X|$. Se um subconjunto $A$ tiver $p$ como palavra associada, cometeremos o abuso de denotar isto por $A=p$. Este tipo de representação será útil também durante a exposição da estrutura de dados, e em particular no exemplo da Figura 5.3.

O exemplo é o contexto dos vertebrados, representado na Tabela 2.1. Suporemos ordenações arbitrárias para $G$ e $M$, descritas a seguir na Figura 5.1.

$$
\begin{aligned}
& M=\left\{m_{1}, m_{2}, m_{3}, m_{4}, m_{5}, m_{6}, m_{7}, m_{8}\right\}, \text { onde: } \\
& m_{1}=\text { esqueleto, } m_{2}=\text { bico, } m_{3}=\text { voa, } m_{4}=\text { mãos, } \\
& m_{5}=\text { vivíparo, } m_{6}=\text { respira na água, } m_{7}=\text { vive na água, } \\
& m_{8}=\text { produz luz. } \\
& G=\left\{g_{1}, g_{2}, g_{3}, g_{4}\right\}, \text { onde: } \\
& g_{1}=\text { peixe lanterna, } g_{2}=\text { águia, } g_{3}=\text { macaco, } g_{4}=\text { peixe papagaio. }
\end{aligned}
$$

Figura 5.1: Conjuntos de objetos e de atributos, ordenados, como exemplo de entrada.

Usando a Tabela 2.1, temos:

$$
\begin{aligned}
& g_{1}^{\prime}=\left\{m_{1}, m_{6}, m_{7}, m_{8}\right\}=10000111 \\
& g_{2}^{\prime}=\left\{m_{1}, m_{2}, m_{3}\right\}=11100000 \\
& g_{3}^{\prime}=\left\{m_{1}, m_{4}, m_{5}\right\}=10011000 \\
& g_{4}^{\prime}=\left\{m_{1}, m_{2}, m_{6}, m_{7}\right\}=11000110 .
\end{aligned}
$$


Antes da primeira iteração do laço da linha 2, temos que $\mathcal{F}=\{M\}=\{11111111\}$. No fim de cada iteração do laço, temos:

$$
\begin{aligned}
& i=1: \\
& \mathcal{F}=\mathcal{F} \cup\left\{g_{1}^{\prime} \cap B \mid B \in \mathcal{F}\right\}=\{11111111,10000111\} \\
& i=2: \\
& \mathcal{F}=\mathcal{F} \cup\left\{g_{2}^{\prime} \cap B \mid B \in \mathcal{F}\right\}=\{11111111,10000111,11100000,10000000\} \\
& i=3: \\
& \mathcal{F}=\mathcal{F} \cup\left\{g_{3}^{\prime} \cap B \mid B \in \mathcal{F}\right\}=\{11111111,10000111,11100000,10000000,10011000\}, \\
& i=4: \\
& \mathcal{F}=\mathcal{F} \cup\left\{g_{4}^{\prime} \cap B \mid B \in \mathcal{F}\right\}=\{1111111,10000111,11100000,10000000,10011000, \\
&11000110,11000000,10000110\} .
\end{aligned}
$$

O leitor pode verificar que o último valor de $\mathcal{F}$ é o conjunto das intenções do contexto mencionado.

\subsubsection{Análise de Complexidade}

A complexidade do Algoritmo 1 dependerá da estrutura de dados utilizada para representarmos $\mathcal{F}$. Em particular, a escolha da estrutura de dados afetará a complexidade computacional associada à execução da linha 5 do Algoritmo 1. Em tal linha, o algoritmo deve decidir se o conjunto intenção pertence a $\mathcal{F}$ e, caso não pertença, deve adicioná-lo a $\mathcal{F}$. Portanto, antes de analisarmos a complexidade deste algoritmo como um todo, mostraremos uma estrutura de dados apropriada para estas duas operações.

Como sabemos, o conjunto $\mathcal{F}$ pode crescer exponencialmente em função dos tamanhos de $G$ e de $M$. Portanto, é de interesse projetar algoritmos cuja complexidade computacional, em função de $\mathcal{F}$, seja a menor possível. Observe que uma implementação que percorra todo o conjunto $\mathcal{F}$ para cada execução da linha 5 , terá complexidade $\Omega\left(|\mathcal{F}|^{2}\right)$. Assim, introduziremos agora uma estrutura de dados que permitirá a execução de tal linha em tempo $O(|M|)$.

Precisaremos de algumas definições preliminares. Uma árvore de raiz $r$ consiste em um grafo $\mathcal{T}=(V, E)$ e um elemento $r \in V$ tal que, para todo $v \in V$, existe exatamente um caminho de $r$ até $v$. Uma folha é um elemento de $V$, diferente de $r$, que sofre a incidência de apenas uma aresta de $E$. Um alfabeto é um conjunto cujos elementos são chamados de símbolos. Sequências de símbolos são chamadas de palavras e, como de costume, estão sujeitas à operação de concatenação.

\section{Árvore de prefixos}

Uma árvore de prefixos é uma quíntupla $(V, E, r, \Sigma, \lambda)$ onde $(V, E, r)$ é uma árvore de raiz $r, \Sigma$ é um alfabeto e $\lambda: E \rightarrow \Sigma$ é uma rotulação das arestas, tal que, para todo $u v, u w \in E$ com $v \neq w$, vale que $\lambda(u v) \neq \lambda(u w)$.

Denotaremos por $\lambda^{V}$ uma rotulação dos vértices, que é induzida por $\lambda$ da seguinte maneira: se $v \in V$, então $\lambda^{V}(v)$ é a concatenação, na ordem natural, dos símbolos presentes nos rótulos do caminho de $r$ até $v$. 
Um exemplo de árvore de prefixos está presente na Figura 5.2. Naquela figura, estão destacados dois vértices, além da raiz, com a finalidade de ilustrar a rotulação de vértices que definimos.

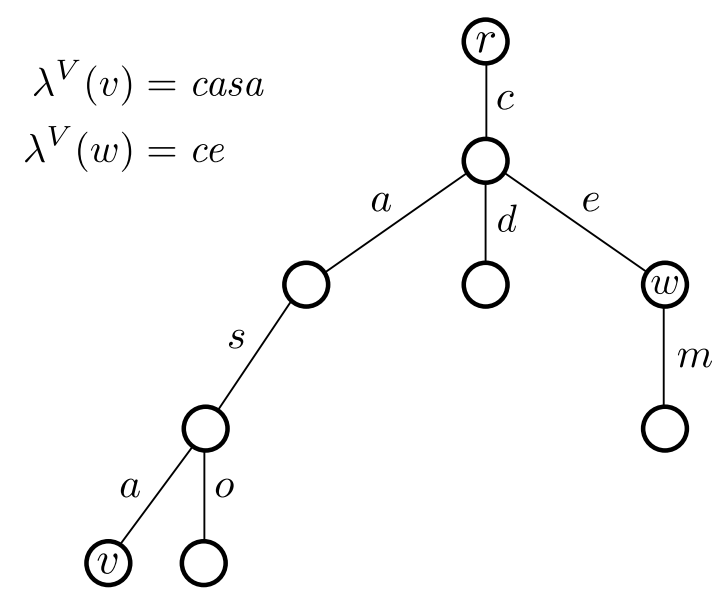

Figura 5.2: Uma árvore de prefixos, com três vértices destacados, e duas de suas rotulações.

\section{Árvore de prefixos de uma família de subconjuntos}

Iremos agora definir uma estrutura que representa as palavras associadas aos subconjuntos de uma família $\mathcal{F} \subseteq \mathcal{P}(M)$, com $M$ ordenado. Seja $\mathcal{F}$ uma família de subconjuntos de um conjunto ordenado $M$. A árvore de prefixos de $\mathcal{F}$ é uma árvore de prefixos $(V, E, r,\{0,1\}, \lambda)$ tal que:

- Para cada $Y \in \mathcal{F}$, existe uma folha $f \in V$ tal que $\lambda^{V}(f)$ é a palavra associada a $Y$.

- Se $f \in V$ for uma folha, então existe $Y \in \mathcal{F}$ cuja palavra associada é $\lambda^{V}(f)$.

Observe que $\left|\lambda^{V}(f)\right|=|M|$ é a distância da raiz a qualquer folha $f$.

Por exemplo, consideremos novamente os atributos do contexto formal dos vertebrados, presente na Tabela 2.1, e tomemos uma família de subconjuntos $\mathcal{F}$ cujos elementos são três intenções daquele contexto:

$$
\mathcal{F}=\{\{\text { esqueleto, mãos, vivíparo }\},\{\text { esqueleto, bico, voa }\}, M\} .
$$

A árvore de prefixos de $\mathcal{F}$ está representada na Figura 5.3. Nesta figura, escolhemos quatro vértices para exibirmos suas rotulações. Três dos quatro vértices escolhidos são folhas, e destacamos quais são os subconjuntos de $M$ associados aos rótulos de tais folhas. O vértice escolhido que não é folha possui como rótulo uma palavra de comprimento um, não havendo, portanto, subconjunto de $M$ associado ao rótulo deste vértice. 


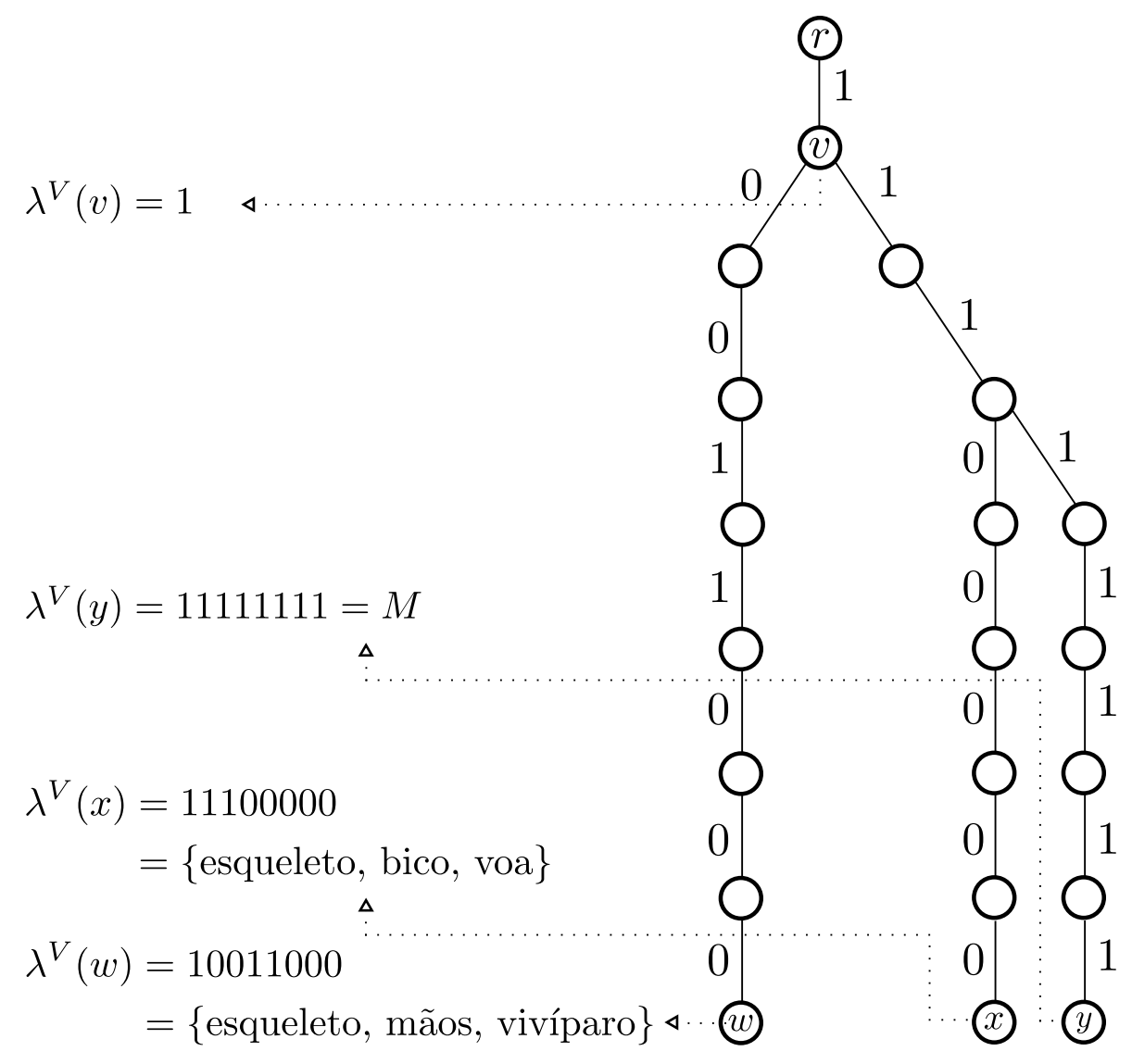

Figura 5.3: Uma árvore de prefixos de uma família de subconjuntos e as rotulações de quatro de seus vértices.

O seguinte lema, cuja demonstração será omitida, segue sem muitas dificuldades a partir do que já foi exposto.

Lema 5.3. Ao serem dados $Y \subseteq M$ e a árvore de prefixos $\mathcal{T}$ de uma família de subconjuntos $\mathcal{F} \subseteq \mathcal{P}(M)$, as operações de:

- Decidir se $Y \in \mathcal{F}$,

- Caso $Y \notin \mathcal{F}$, adicionar um vértice $v$ a $\mathcal{T}$ de maneira que $\lambda^{V}(v)=Y$,

podem ser executadas em tempo $O(|M|)$.

A complexidade de tempo de uma implementação do Algoritmo 1 que utilize uma árvore de prefixos será mostrada no próximo teorema. A obtenção de $\mathfrak{B}(\mathbb{K})$ a partir de $\operatorname{Int}(\mathbb{K})$ também é detalhada.

Teorema 5.4. Com a utilização de uma árvore de prefixos, o Algoritmo 1 pode ser implementado de maneira a encontrar o conjunto das intenções de um contexto $(G, M, I) \mathrm{em}$ tempo $O(|\mathfrak{B}| \cdot|G| \cdot|M|)$. O pós-processamento que obtém o conjunto de conceitos a partir do conjunto das intenções pode ser realizado com a mesma complexidade de tempo. 
Prova. Uma execução da linha 4 do Algoritmo 1 consome $O(|M|)$ unidades de tempo, que é o tempo necessário para o cálculo da intenção-objeto $g^{\prime}$, e também o tempo necessário para calcular a intersecção de dois subconjuntos de $M$. Com relação à linha 5, o Lema 5.3 garante uma complexidade de tempo $O(|M|)$ se representarmos $\mathcal{F}$ através de uma árvore de prefixos. Portanto, o Algoritmo 1 consome tempo $O(|\mathfrak{B}| \cdot|G| \cdot|M|)$.

O pós-processamento mencionado, cuja finalidade é obter o conjunto de todos os conceitos, pode claramente ser realizado em tempo $O(|\mathfrak{B}| \cdot|G| \cdot|M|)$, bastando para isto, derivar em tempo $O(|G||M|)$ cada uma das $|\mathfrak{B}|$ intenções devolvidas pelo Algoritmo 1.

\subsection{Algoritmo de Ganter}

Nesta seção, exporemos o algoritmo conhecido como algoritmo de Ganter (e também como NextClosure), publicado em [Gan84].

Recordamos que um operador de fecho é uma função $c l: \mathcal{P}(S) \rightarrow \mathcal{P}(S)$ satisfazendo, para quaisquer $P, Q \subseteq S$, as propriedades de monotonicidade crescente, extensividade e idempotência. Isto é, respectivamente, $P \subseteq Q \Rightarrow \operatorname{cl}(P) \subseteq \operatorname{cl}(Q), P \subseteq \operatorname{cl}(P)$ e, por fim, $\operatorname{cl}(\operatorname{cl}(P))=\operatorname{cl}(P)$ para todo $P, Q \subseteq S$.

Vimos na Proposição 2.2 que extensões e intenções de um contexto são fechados, respectivamente, dos operadores de fecho " $: \mathcal{P}(G) \rightarrow \mathcal{P}(G) \mathrm{e}^{\prime \prime}: \mathcal{P}(M) \rightarrow \mathcal{P}(M)$. O algoritmo de Ganter encontra os fechados de um operador de fecho qualquer $c l$, e, portanto, pode encontrar as extensões (ou as intenções) de um contexto. Este algoritmo possui a boa propriedade de ser um algoritmo de atraso polinomial (polynomial delay algorithm). Além disso, o espaço adicional $^{2}$ necessário a esta solução é baixo, uma vez que não é necessária a manutenção de uma lista com os fechados já encontrados. Mais precisamente, o espaço adicional utilizado por este algoritmo é $O(|G|+|M|)$, enquanto que no Algoritmo 1 é $\Omega(|\mathfrak{B}|)$.

Seja $S$ um conjunto ordenado. Suporemos, a fim de simplificar a exposição, que tal conjunto é $S=\{1,2, \ldots, n\}$, equipado com a ordem usual. Vamos definir uma ordenação total estrita sobre $\mathcal{P}(S)$, que chamaremos de ordem lexicográfica. Para dois subconjuntos $P$ e $Q$ de $S$, definimos $P<Q$ se e somente se $P \neq Q$ e o menor elemento de $S$ que pertence à diferença simétrica $P \triangle Q$ pertence a $Q$. Isto é, formalmente:

$$
P<Q \Leftrightarrow \exists i \in Q \backslash P: P \cap\{1,2, \ldots, i-1\}=Q \cap\{1,2, \ldots, i-1\} .
$$

Observe que < é uma ordem total estrita sobre $\mathcal{P}(S)$, pois para cada $P, Q \subseteq S$, distintos, vale que $P<Q$ ou $Q<P$. A menos que seja dito explicitamente o contrário, esta é a ordem que usaremos em $\mathcal{P}(S)$. Para cada $i \in S$, definimos uma ordem parcial estrita $<_{i}$, da seguinte maneira: $P<_{i} Q$ se e só se $P<Q$ e o menor elemento de $S$ que difere $Q$ de $P$ é $i$, ou seja:

$$
P<_{i} Q \Leftrightarrow i \in Q \backslash P: P \cap\{1,2, \ldots, i-1\}=Q \cap\{1,2, \ldots, i-1\} .
$$

Note que $P<_{i} Q \Rightarrow P<Q$.

Sejam $c l: \mathcal{P}(S) \rightarrow \mathcal{P}(S)$ um operador de fecho, $P \subseteq S$ e $i \in S$. Definimos, ainda:

$$
P \oplus i=c l(P \cap\{1,2, \ldots, i-1\} \cup\{i\}) .
$$

\footnotetext{
${ }^{2}$ Isto é, o espaço necessário além do gasto para se representar a entrada.
} 
Convém observar que, pela idempotência de $c l$, o conjunto $P \oplus i$ é sempre um fechado de $c l$. A estratégia do algoritmo de Ganter é encontrar os fechados de $c l$ na ordem < que acabamos de definir. A cada iteração é encontrado o sucessor do último fechado descoberto. Mostraremos um teorema que caracteriza o fechado que é sucessor de um subconjunto $P \subsetneq S$ arbitrário. Além disso, tal teorema afirma que o menor dos fechados é sempre $c l(\emptyset)$, e o maior, $S$. Feitas essas considerações, a estratégia do algoritmo de Ganter delinea-se da seguinte maneira: o primeiro fechado descoberto pelo algoritmo é $c l(\emptyset)$. A partir de então, sucessivamente, o algoritmo encontra o fechado que é sucessor do último fechado já obtido, através da caracterização mencionada.

Antes de mostrarmos o teorema que caracteriza o sucessor segundo a ordem $<$, precisamos mostrar algumas propriedades envolvendo as ordens $<\mathrm{e}<_{i}$, bem como a operação $\oplus$.

Proposição 5.5. Sejam $P, Q, R \subseteq S, c l: \mathcal{P}(S) \rightarrow \mathcal{P}(S)$ um operador de fecho $e \oplus$ a operação acima definida. Então valem:

1) $P<Q \Leftrightarrow P<{ }_{i} Q$ para exatamente um $i \in S$.

2) $P<_{i} Q, P<_{j} R$ e $i<j \Rightarrow R<_{i} Q$.

3) $P<_{i} Q$ e $Q=\operatorname{cl}(Q) \Rightarrow P \oplus i \subseteq Q \Rightarrow P \oplus i \leq Q$.

4) $P<_{i} Q$ e $Q=\operatorname{cl}(Q) \Rightarrow P<_{i} P \oplus i$.

Prova. 1) A direção $\Leftarrow$ segue da definição. Com relação à outra direção, suponha que $P<Q$. Então existe $i$ tal que $P<_{i} Q$. Seja, então, $i$ o menor inteiro tal que $P<_{i} Q$. Como $i \in P \backslash Q$, temos claramente que:

$$
P \cap\{1,2, \ldots, j-1\} \neq Q \cap\{1,2, \ldots, j-1\}, \text { para todo } j \geq i+1 .
$$

Então, para todo $j \geq i+1$, não vale que $P<_{j} Q$, e portanto existe um único $i$ satisfazendo $P<_{i} Q$.

2) Suponha que $P<_{i} Q, P<_{j} R$ e que $i<j$. Pela definição da ordem $<_{k}$, temos que:

$$
P<_{i} Q \Rightarrow\left\{\begin{array}{l}
i \in Q \\
i \notin P \\
P \cap\{1,2, \ldots, i-1\}=Q \cap\{1,2, \ldots, i-1\} .
\end{array}\right.
$$

Assim como:

$$
P<{ }_{j} R \Rightarrow\left\{\begin{array}{l}
j \in Q \\
j \notin P \\
P \cap\{1,2, \ldots, j-1\}=R \cap\{1,2, \ldots, j-1\} .
\end{array}\right.
$$

De (5.2) e de $i<j$, temos que $P \cap\{1,2, \ldots, i-1\}=R \cap\{1,2, \ldots, i-1\}$. Agora, por transitividade e (5.1), segue que

$$
R \cap\{1,2, \ldots, i-1\}=Q \cap\{1,2, \ldots, i-1\} .
$$


Como $P<_{j} R, j \neq i$ e o item 1$)$ garante que o inteiro $j$ tal que $P<_{j} R$ é único, temos que não vale que $P<_{i} R$. Agora, afirmamos que

$$
i \notin R
$$

De fato, pois, caso contrário valeria que $P<_{i} R$, uma vez que temos que $i \notin P$, por (5.1), e também que $P \cap\{1,2, \ldots, i-1\}=R \cap\{1,2, \ldots, i-1\}$, por (5.2) e $i<j$.

Por outro lado, a hipótese de $P<_{i} Q$ implica que $i \in Q$, e assim, $i \in Q \backslash R$. A validade de $i \in Q \backslash R$ em conjunto com (5.3) equivale a $R<_{i} Q$.

3) Suponha que $P<_{i} Q$ e que $Q=\operatorname{cl}(Q)$. Então:

$$
P<_{i} Q \Rightarrow\left\{\begin{array}{l}
P \cap\{1,2, \ldots, i-1\}=Q \cap\{1,2, \ldots, i-1\} \\
i \in Q \backslash P
\end{array}\right.
$$

Portanto, claramente $P \cap\{1,2, \ldots, i-1\} \subseteq Q$. Como $i \in Q$, vale que

$$
(P \cap\{1,2, \ldots, i-1\}) \cup\{i\} \subseteq Q .
$$

Como $c l$ é operador de fecho, podemos aplicá-lo aos dois lados de (5.4), e a inclusão se mantém:

$$
\begin{aligned}
c l((P \cap\{1,2, \ldots, i-1\}) \cup\{i\}) & \subseteq \operatorname{cl}(Q) \\
\Leftrightarrow P \oplus i & \subseteq \operatorname{cl}(Q)=Q \quad \text { (pela definição de } P \oplus i) .
\end{aligned}
$$

Assim, segue a primeira parte da tese. Agora mostraremos que $P \oplus i \leq Q$. Como acabamos de mostrar, $P \oplus i \subseteq Q$. Ou seja,

$$
(P \oplus i) \backslash Q=\emptyset
$$

Logo, não existe $i$ tal que $Q<_{i} P \oplus i$, e portanto não vale que $Q<P \oplus i$, pelo item 1 ). Mas, como < é ordem total, vale necessariamente que $P \oplus i<Q$ ou que $P \oplus i=Q$, ou seja, $P \oplus i \leq Q$.

4) Suponha que $P<_{i} Q$ e que $Q=\operatorname{cl}(Q)$. Vamos mostrar inicialmente que

$$
P \cap\{1,2, \ldots, i-1\}=(P \oplus i) \cap\{1,2, \ldots, i-1\} .
$$

Para tanto, mostraremos as duas inclusões em questão. Claramente, temos que

$$
\begin{aligned}
P \cap\{1,2, \ldots, i-1\} & \subseteq(P \cap\{1,2, \ldots, i-1\}) \cup\{i\} \\
& \subseteq c l((P \cap\{1,2, \ldots, i-1\}) \cup\{i\})=P \oplus i \quad(c l \text { é op. de fecho }) .
\end{aligned}
$$

Consequentemente, segue que $P \cap\{1,2, \ldots, i-1\} \subseteq P \oplus i$. Como evidentemente vale que $P \cap\{1,2, \ldots, i-1\} \subseteq\{1,2, \ldots, i-1\}$, temos então que

$$
P \cap\{1,2, \ldots, i-1\} \subseteq(P \oplus i) \cap\{1,2, \ldots, i-1\} .
$$


Agora, para a inclusão contrária, aplicamos o item 3), usando o fato de que, por hipótese, $P<_{i} Q$ e $Q=\operatorname{cl}(Q)$. Assim, obtemos que $P \oplus i \subseteq Q$. Portanto,

$$
(P \oplus i) \cap\{1,2, \ldots, i-1\} \subseteq Q \cap\{1,2, \ldots, i-1\} .
$$

Como $P<_{i} Q$, vale que

$$
Q \cap\{1,2, \ldots, i-1\}=P \cap\{1,2, \ldots, i-1\}
$$

Utilizando (5.6), (5.7) e a transitividade da relação $\subseteq$, obtemos:

$$
(P \oplus i) \cap\{1,2, \ldots, i-1\} \subseteq P \cap\{1,2, \ldots, i-1\}
$$

Logo, a equação (5.5) vale. Como por hipótese, temos que $P<_{i} Q$, então $i \notin P$. Por outro lado, claramente $i \in P \oplus i$, e portanto, $i \in(P \oplus i) \backslash P$. Este último fato em conjunto com (5.5) equivale a $P<_{i} P \oplus i$.

O próximo teorema caracteriza o fechado de $c l$ que é sucessor de um subconjunto próprio de $S$, e também quais fechados são o mínimo e o máximo em $\mathcal{P}(S)$.

Teorema 5.6. Sejam $S$ um conjunto ordenado e cl $: \mathcal{P}(S) \rightarrow \mathcal{P}(S)$ um operador de fecho. Dado $P \subsetneq S$, o menor fechado maior que $P$ é

$$
P \oplus i
$$

onde $i$ é o maior elemento de $S$ satisfazendo $P<_{i} P \oplus i$. Ademais, o menor de todos os fechados é cl $(\emptyset)$, e, o maior de todos os fechados, $S$.

Prova. Vamos mostrar primeiramente que $c l(\emptyset)$ é o menor dos fechados, segundo a ordem lexicográfica. Seja $P$ um fechado de $c l$, isto é, $P=c l(P)$. Claramente:

$$
\emptyset \subseteq P \Rightarrow \operatorname{cl}(\emptyset) \subseteq \operatorname{cl}(P)=P
$$

e, assim, $\operatorname{cl}(\emptyset) \backslash P=\emptyset$. Da definição de $<$, segue que $P \nless c l(\emptyset)$. Como a ordem $<$ é total (isto é, uma cadeia), temos que $\operatorname{cl}(\emptyset) \leq P$. Analogamente segue que $S=\operatorname{cl}(S)$ é o maior fechado de $c l$.

Agora, seja $P^{+}$o menor fechado maior que $P$. Já que $P<P^{+}$, podemos tomar $i \in S$ com $P<_{i} P^{+}$, pelas definições das ordens $<\mathrm{e}<_{k}$. Então:

$$
\begin{aligned}
P<_{i} P^{+} & \Rightarrow P<_{i} P \oplus i \quad \text { (pelo item 4), Proposição 5.5) } \\
& \Rightarrow P<P \oplus i .
\end{aligned}
$$

Por outro lado, o fato de $P<_{i} P^{+}$implica, pelo item 3) da Proposição 5.5, que $P \oplus i \leq P^{+}$. Logo,

$$
P<P \oplus i \leq P^{+} .
$$


Da escolha de $P^{+}$, e pelo fato de $P \oplus i$ ser fechado (pois $c l$ é idempotente), segue necessariamente que $P \oplus i=P^{+}$. Resta provar que não existe $j>i$ satisfazendo $P<_{j} P \oplus j$. Suponha, por absurdo, que exista um tal $j$. Assim:

$$
\left.\begin{array}{c}
P<_{i} P \oplus i \\
P<_{j} P \oplus j \\
j>i
\end{array}\right\} \Rightarrow P \oplus j<_{i} P \oplus i \quad \text { (pelo item 2), Proposição 5.5). }
$$

Então, novamente, pelas definições das ordens $<$ e $<_{k}$, segue que $P \oplus j<P \oplus i$. Da mesma maneira, $P<{ }_{j} P \oplus j$ claramente implica que $P<P \oplus j$. Assim,

$$
P<P \oplus j<P \oplus i=P^{+},
$$

o que contradiz a escolha de $P^{+}$.

O algoritmo de Ganter está descrito no Algoritmo 2. Sua entrada é composta por um conjunto ordenado $S$ e um operador de fecho $c l: \mathcal{P}(S) \rightarrow \mathcal{P}(S)$. Note que, a cada descoberta de um fechado, podemos imprimí-lo na saída, e que não são armazenados todos os fechados já descobertos até então, mas apenas o último.

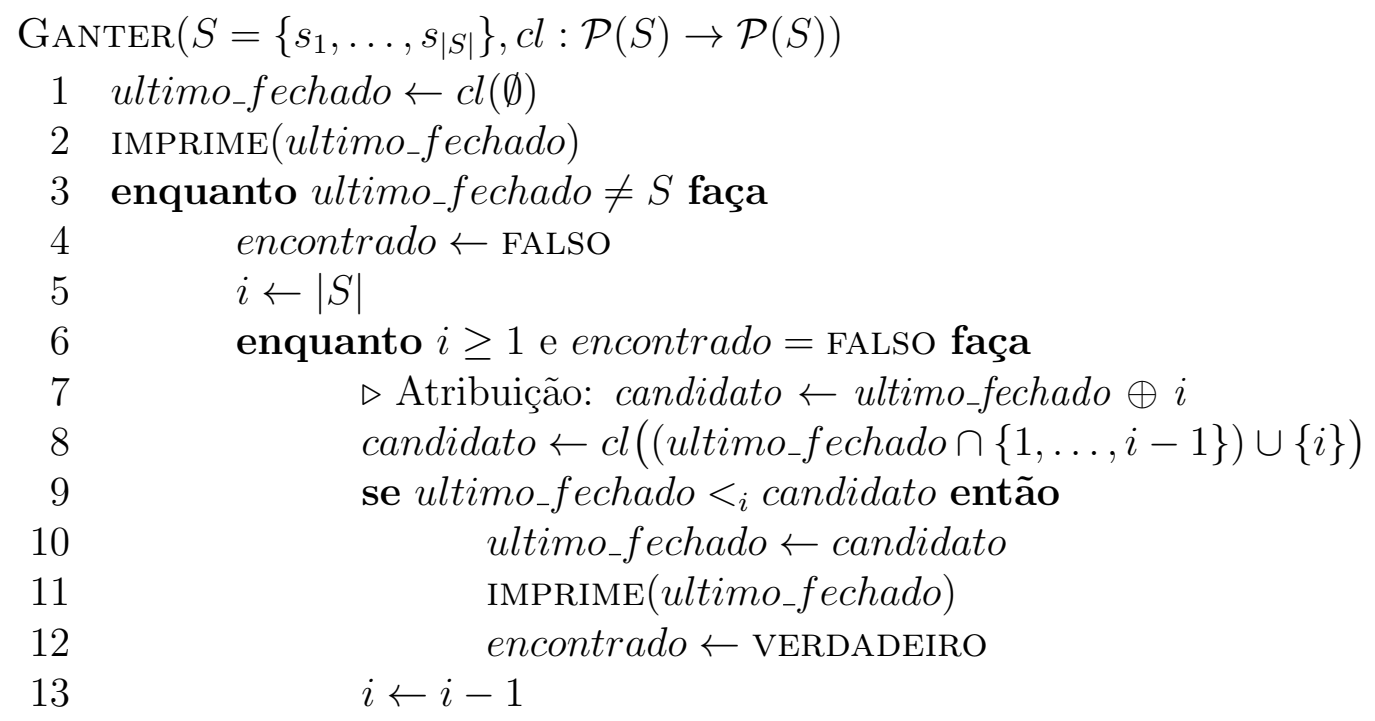

Algoritmo 2: Algoritmo de Ganter, para a descoberta dos fechados de um operador de fecho.

\section{Um exemplo de execução}

Daremos um exemplo de algumas iterações da execução do Algoritmo 2, novamente tomando como exemplo o contexto dos vertebrados, representado na Tabela 2.1. Como entrada, usamos $M$, sob a mesma ordenação do exemplo de execução do Algoritmo 1, que está representada na Figura 5.1, e $\mathrm{cl}$ como sendo o operador de derivação dupla sobre conjuntos de atributos, " $: \mathcal{P}(M) \rightarrow \mathcal{P}(M)$. Note que $\operatorname{cl}(\emptyset)=10000000$. Por motivos de espaço, na Tabela 5.1, abreviamos o nome da variável ultimo_fechado para ult_fechado, candidato para cand., e a coluna pré fecho se refere ao valor de (ultimo_fechado $\cap\{1, \ldots, i-1\}) \cup\{i\}$. 


\begin{tabular}{|c|c|c|c|c|c|}
\hline ult_fechado & $i$ & pré fecho & cand. & ult_fechado $<_{i}$ cand. $?$ & ação \\
\hline 10000000 & 8 & 10000001 & 10000111 & não & \\
\hline 10000000 & 7 & 10000010 & 10000110 & não & \\
\hline 10000000 & 6 & 10000100 & 10000110 & sim & imprime 10000110 \\
\hline 10000110 & 8 & 10000111 & 10000111 & sim & imprime 10000111 \\
\hline 10000111 & 8 & 10000111 & 10000111 & não & \\
\hline 10000111 & 7 & 10000110 & 10000110 & não & \\
\hline 10000111 & 6 & 10000100 & 10000110 & não & \\
\hline 10000111 & 5 & 10001000 & 10011000 & não & \\
\hline 10000111 & 4 & 10010000 & 10011000 & sim & imprime 10011000 \\
\hline$\vdots$ & & & & & \\
\hline
\end{tabular}

Tabela 5.1: Exemplo de execução do Algoritmo 2.

\subsubsection{Análise de complexidade}

Faremos uma análise de complexidade de tempo do Algoritmo 2. Seja $F$ o conjunto de fechados de $c l$. O número de iterações do laço presente na linha 3 é igual a $|F|-1$, já que $c l(\emptyset)$ é descoberto pela execução da linha 1 e, a cada iteração do laço em questão, temos um novo fechado sendo descoberto. A linha 8 pode ser trivialmente implementada e, ainda assim, consumir tempo $O(\max \{|S|$, custo $(c l)\})$, onde custo $(c l)$ é o tempo necessário para o cálculo de $c l$. As linhas 10, 11 e 12 consomem tempo $O(|S|)$. Portanto, concluímos que a complexidade de tempo do algoritmo como um todo é $O(|F| \cdot|S| \cdot \max \{|S|$, custo $($ cl $)\}$ ).

Para o caso particular onde $c l: \mathcal{P}(M) \rightarrow \mathcal{P}(M)$ é o operador de derivação dupla, temos um algoritmo de tempo $O\left(|\mathfrak{B}| \cdot|G| \cdot|M|^{2}\right)$. No que se refere a espaço consumido, conforme ressaltamos, não é necessária a manutenção de uma lista dos fechados (isto é, neste caso, as intenções) já descobertos. Assim, o espaço necessário para armazenar a última intenção é $O(|M|)$. Além disso, ao implementarmos o operador de derivação dupla de maneira iterada, temos que esta aplicação de $c l$ consome espaço $O(|G|+|M|)$, pois o resultado de uma aplicação da derivação de objetos é um conjunto de atributos, e este deve ser armazenado para a segunda aplicação de derivação. Logo, o consumo de espaço deste caso particular do Algoritmo 2 é $O(|G|+|M|)$, sem contarmos o espaço necessário para se representar a entrada.

Com relação ao atraso do Algoritmo 2, isto é, o tempo necessário até a impressão do primeiro fechado, assim como o tempo necessário entre a impressão do último fechado e o encerramento do algoritmo, bem como o tempo necessário entre quaisquer duas impressões de fechados, temos que esta grandeza é limitada em $O(|S| \cdot \max \{|S|$, custo $($ cl $)\})$. No caso particular da determinação das intenções, o Algoritmo 2 apresenta atraso $O\left(|G| \cdot|M|^{2}\right)$.

\subsubsection{Adaptação para a determinação dos conceitos}

Durante a exposição do Algoritmo 1, argumentamos que um pós processamento é suficiente para obter-se, com mesma complexidade de tempo, o conjunto de conceitos a partir do conjunto das intenções. Para obtermos o conjunto de conceitos, ao invés do conjunto das 
intenções, não consideraremos, no caso deste algoritmo, um pós processamento. Ao invés, expomos o Algoritmo 3, que se trata de uma aplicação e pequena modificação do Algoritmo 2. É fácil observar que tal algoritmo apresenta mesma complexidade de tempo e espaço com relação ao algoritmo original no caso em que $c l=$ ".

Nas linhas 8 e 9 do algoritmo modificado, a aplicação do operador de derivação dupla " é realizada em dois passos, armazenando em uma variável temporária o valor da primeira derivação. Seja $P$ o valor assumido pela variável ultimo_fechado naquele algoritmo. Toda vez que um candidato $P \oplus i$ é submetido ao teste $P<_{i} P \oplus i$, já se encontra armazenada em uma variável temporária o valor da derivação de $P \cap\{1,2, \ldots, i-1\} \cup\{i\}$. No caso de $P \oplus i$ ser uma intenção, isto é, no caso de $P \oplus i$ validar o teste da linha 11, o valor desta variável é claramente a extensão associada à intenção $P \oplus i$.

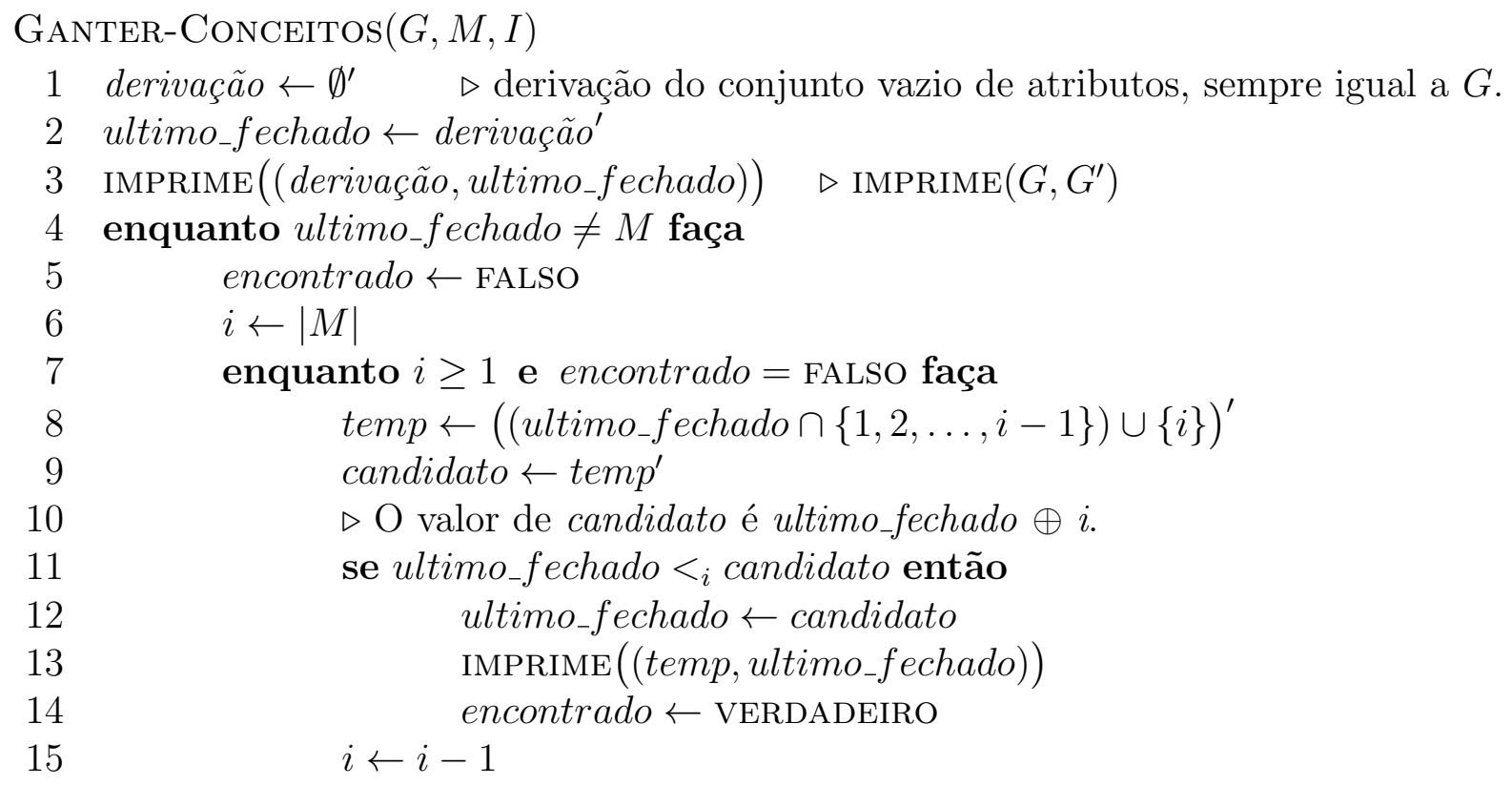

Algoritmo 3: Algoritmo de Ganter, aplicado à determinação dos conceitos. 


\section{Capítulo 6}

\section{Determinação do reticulado de conceitos}

Neste capítulo, consideramos o problema de se encontrar o diagrama de Hasse de um reticulado de conceitos de um contexto finito. Apresentaremos dois algoritmos para este problema, respectivamente nas Seções 6.1 e 6.2. O primeiro algoritmo que será exposto pode ser entendido como uma criação do diagrama de $\underline{\mathfrak{B}}(\mathbb{K})$ por níveis, a partir do seu elemento máximo. Veremos que esta solução apresenta complexidade de tempo $O\left(|\mathfrak{B}| \cdot|G| \cdot|M|^{2}\right)$.

O segundo algoritmo resolve o problema de se encontrar o diagrama de Hasse de um fecho por uniões. Fechos por uniões podem ser entendidos como duais dos fechos por intersecções, que foram objetos tratados durante a exposição do Algoritmo 1, realizada na Seção 5.1. Naquela ocasião, vimos que cada intenção $B$ pode ser escrita como $B=\bigcap_{g \in S} g^{\prime}$. Portanto, uma utilização elementar da lei de de Morgan implica que cada complemento de intenção, $\bar{B}$, pode ser escrito como $\bar{B}=\bigcup_{g \in S} \overline{g^{\prime}}$. Sem muitas dificuldades, também pode se mostrar que o conjunto de complementos de intenções também é um reticulado, e sempre dual (no sentido de ordem parcial) ao reticulado de intenções, o que estabelece uma caracterização de uma estrutura dual ao reticulado de intenções, em termos de uniões. Esta caracterização será vital para mostrarmos que o segundo algoritmo mostrado neste capítulo pode ser usado para resolver o problema de se encontrar o reticulado de conceitos. Em termos de complexidade, o tempo gasto por este algoritmo é $O(|\mathfrak{B}| \cdot|G| \cdot|M|)$.

\subsection{Algoritmo dos Próximos Vizinhos}

Seja $\mathbb{K}=(G, M, I)$ um contexto finito. O algoritmo Próximos Vizinhos encontra o diagrama de $\underline{\mathfrak{B}}(\mathbb{K})$ pelo que chamaremos de níveis. Definimos que o nível de um conceito $(A, B)$ é o menor $k$ tal que $(A, B)=\left(A_{1}, B_{1}\right) \prec\left(A_{2}, B_{2}\right) \prec \ldots \prec\left(A_{k}, B_{k}\right)=\left(G, G^{\prime}\right)$, para conceitos $\left(A_{i}, B_{i}\right) \in \mathfrak{B}(\mathbb{K}), i=1,2, \ldots, k$. Isto é, o tamanho (em número de vértices) do menor caminho orientado de $(A, B)$ a $\left(G, G^{\prime}\right)$ no diagrama de Hasse de $\underline{\mathfrak{B}}(\mathbb{K})$. Note que o nível do conceito $\left(G, G^{\prime}\right)$, isto é, do elemento máximo de $\underline{B}(\mathbb{K})$, é um.

De importância central para este algoritmo é a rotina que chamaremos de EncontraVizinhosDeBaixo. Esta rotina recebe um conceito $(A, B) \in \underline{\mathfrak{B}}(G, M, I)$ e encontra todos os conceitos que são cobertos por ele. A estratégia do algoritmo Próximos Vizinhos é a seguinte: 
1. O primeiro nível é trivialmente encontrado: $\left\{\left(G, G^{\prime}\right)\right\}$.

2. Após ser encontrado o $i$-ésimo nível, $\left\{\left(A_{1}, B_{1}\right),\left(A_{2}, B_{2}\right), \ldots,\left(A_{k}, B_{k}\right)\right\}$, o nível de número $i+1$ é encontrado ao aplicar-se a rotina Encontra VizinhosDeBaixo para cada elemento $\left(A_{i}, B_{i}\right), \operatorname{com} i=1, \ldots, k$.

Vamos mostrar uma proposição que descreve um conjunto de candidatos a cobrirem um conceito $(A, B) \in \underline{\mathfrak{B}}(G, M, I)$. Veremos que este conjunto de candidatos apresenta a boa propriedade de possuir $O(|M|)$ elementos, e que este será de grande utilidade para a rotina EncontraVizinhosDeBaixo, a ser descrita.

Proposição 6.1. Sejam $\underline{\mathfrak{B}}(G, M, I)$ um reticulado de conceitos e $(A, B)$ um de seus conceitos. Todos os conceitos cobertos por $(A, B)$ possuem extensão pertencente à família de subconjuntos

$$
\mathcal{L}=\left\{(B \cup\{m\})^{\prime} \mid m \in M \backslash B\right\} .
$$

Ademais, todo subconjunto em $\mathcal{L}$ é uma extensão contida propriamente em $A$.

Prova. Seja $(A, B)$ um conceito, e seja $\mathcal{L}$ como no enunciado. Seja, ainda, $(X, Y)$ um conceito coberto por $(A, B)$ e, portanto, $Y \supsetneq B$. Seja $m \in Y \backslash B$. Definimos $W=B \cup\{m\}$. Note que $B \subsetneq W \subseteq Y$. Consideramos agora o par $\left(W^{\prime}, W^{\prime \prime}\right)$. Pela Proposição 2.3, item iii'), temos que $\left(W^{\prime}, W^{\prime \prime}\right)$ é um conceito. Ademais, temos: $W \supsetneq B \Rightarrow W^{\prime \prime} \supseteq B^{\prime \prime}=B$ e, como $m \in W^{\prime \prime} \backslash B$, temos, na realidade, a inclusão estrita: $W^{\prime \prime} \supsetneq B$. Portanto, $\left(W^{\prime}, W^{\prime \prime}\right)<(A, B)$. Por outro lado, já que $W \subseteq Y$, também temos que $W^{\prime \prime} \subseteq Y^{\prime \prime}=Y$ e, portanto $(X, Y) \leq\left(W^{\prime}, W^{\prime \prime}\right)$. Em suma, temos:

$$
(X, Y) \leq\left(W^{\prime}, W^{\prime \prime}\right)<(A, B) .
$$

Como $(X, Y)$ é coberto por $(A, B)$, a desigualdade $(X, Y) \leq\left(W^{\prime}, W^{\prime \prime}\right)$ vale com igualdade e portanto $X=W^{\prime}=(B \cup\{m\})^{\prime}$ e, consequentemente, $X$ pertence a $\mathcal{L}$.

Para mostrarmos que todo subconjunto em $\mathcal{L}$ é uma extensão contida propriamente em $A$, tomamos $Z \in \mathcal{L}$, e portanto $Z$ é da forma $(B \cup\{m\})^{\prime}$, para algum $m \in M \backslash B$. Pela Proposição 2.3, item iii), e pela Proposição 2.2, item ii), segue que $Z$ é uma extensão. Usando o Lema 2.6, temos:

$$
\begin{aligned}
Z & =(B \cup\{m\})^{\prime} \\
& =B^{\prime} \cap\{m\}^{\prime}=A \cap\{m\}^{\prime} .
\end{aligned}
$$

Logo, $Z \subseteq A$. Como $m \notin B$, segue que $m \notin A^{\prime}$, e portanto existe $g \in A$ tal que $g \mp m$, consequentemente $\{m\}^{\prime} \nsupseteq A$ e, assim, $Z \subsetneq A$.

A recíproca da afirmação contida na Proposição 6.1, no entanto, não vale: nem todo elemento da família $\mathcal{L}$ presente no enunciado é uma extensão cujo conceito associado é coberto por $(A, B)$. Por exemplo, tomamos novamente o contexto dos vertebrados, e o conceito $(A, B)=(\{$ peixe papagaio, águia, macaco, peixe lanterna $\}$, \{esqueleto $\})$. Tomamos também um elemento da família $\mathcal{L}: X=(B \cup\{\text { produz luz }\})^{\prime}$. A Figura 6.1(a) mostra que $(B \cup\{\text { produz luz }\})^{\prime}=\{$ peixe lanterna $\}$, enquanto que a Figura 6.1(b) mostra o conjunto $\{\text { peixe lanterna }\}^{\prime}=X^{\prime}$. No entanto, é mostrado na Figura 6.2 que o conceito $\left(X, X^{\prime}\right)$ não é coberto por $(A, B)$. 


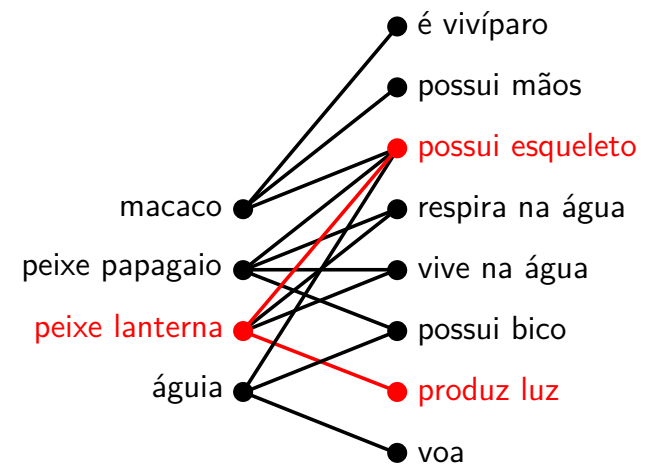

(a) Derivação de \{possui esqueleto, produz luz\}

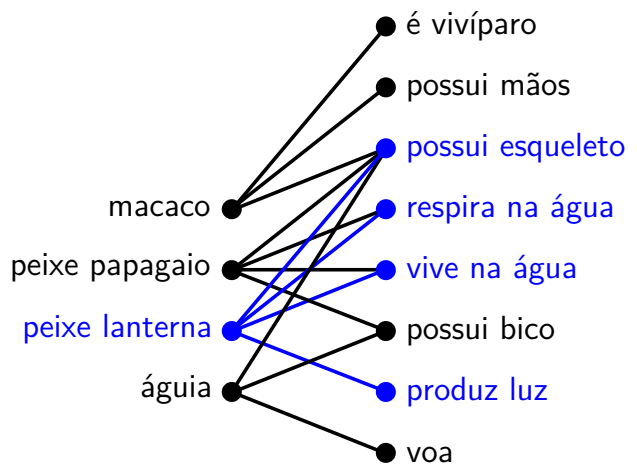

(b) Derivação de \{peixe lanterna\}

Figura 6.1: Conceito do contra-exemplo para a recíproca da Proposição 6.1.

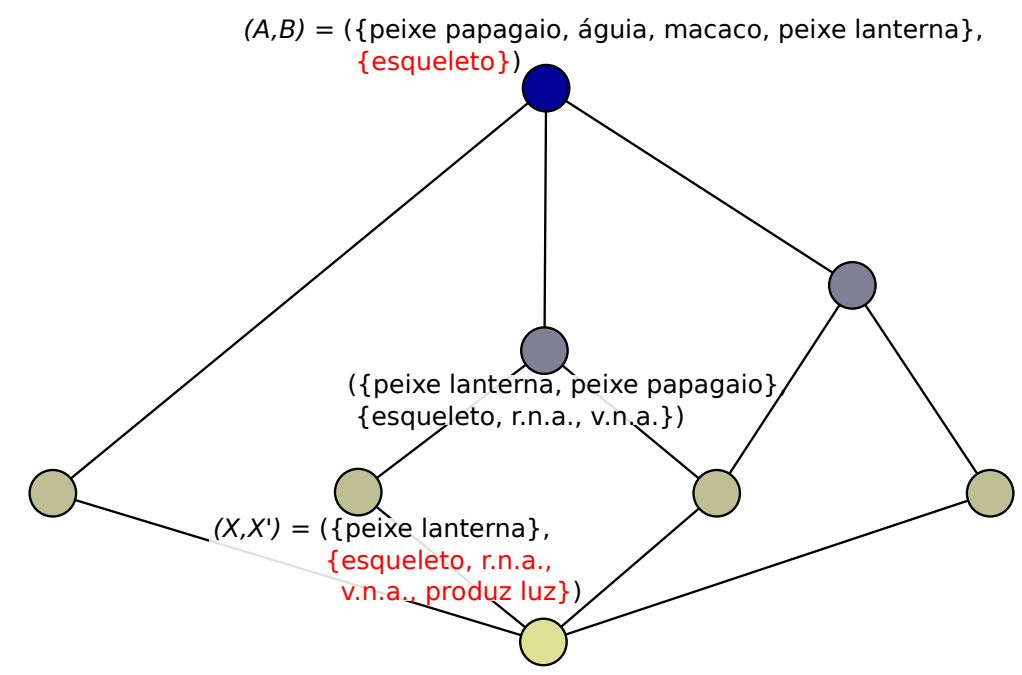

Figura 6.2: Contra-exemplo para a recíproca da Proposição 6.1.

A não validade da recíproca significa que devemos submeter $\mathcal{L}$ a um processo que remova de $\mathcal{L}$ extensões que não são de conceitos cobertos por $(X, Y)$. Na Proposição 6.2, vamos mostrar que as extensões pertencentes a $\mathcal{L}$ que devem ser removidas são exatamente as não maximais em $\mathcal{L}$.

Proposição 6.2. Sejam $\mathbb{K}=(G, M, I)$ um contexto e $(A, B) \in \mathfrak{B}(G, M, I)$ um conceito qualquer. Seja $\mathcal{L}$ a familia de candidatos definida como na Proposição 6.1. Seja $(X, Y)$ um conceito cuja extensão pertence a $\mathcal{L}$, isto é, $X=(B \cup\{m\})^{\prime}$, para algum $m \in M \backslash B$. Então, o conceito $(A, B)$ cobre $(X, Y)$ se e somente se $X$ é maximal em $\mathcal{L}$.

Prova. Vamos provar que, se $X$ não é maximal em $\mathcal{L}$, então $(A, B)$ não cobre $(X, Y)$. Suponha que $X$ não seja maximal em $\mathcal{L}$, e seja $X_{1} \in \mathcal{L}$ tal que $X \subsetneq X_{1}$. Pela Proposição 6.1, temos que $X_{1} \subsetneq A$ e que $X_{1}$ é uma extensão. Portanto, $\left(X_{1}, X_{1}^{\prime}\right)$ é um conceito. Além disso, temos $(X, Y)<\left(X_{1}, X_{1}^{\prime}\right)<(A, B)$, e portanto $(A, B)$ não cobre $(X, Y)$. 
Para a recíproca, vamos mostrar que se $(A, B)$ não cobre $(X, Y)$, então $X$ não é maximal em $\mathcal{L}$. Suponha, então, que $(A, B)$ não cobre $(X, Y)$, e tome $(Z, W) \in \mathfrak{B}(G, M, I)$ tal que $(X, Y)<(Z, W)<(A, B)$. Como $(Z, W)<(A, B)$, segue imediatamente que $W \supsetneq B$. Logo, podemos tomar $m \in W \backslash B$. Definimos $W_{1}=B \cup\{m\}$. Note que $W_{1}^{\prime} \in \mathcal{L}$ e que $W_{1} \subseteq W$. Vale que:

$$
W_{1} \subseteq W \Rightarrow W_{1}^{\prime \prime} \subseteq W^{\prime \prime}=W \Rightarrow(Z, W) \leq\left(W_{1}^{\prime}, W_{1}^{\prime \prime}\right) .
$$

Como $(X, Y)<(Z, W)$, temos, por transitividade, $(X, Y)<\left(W_{1}^{\prime}, W_{1}^{\prime \prime}\right) \Leftrightarrow X \subsetneq W_{1}^{\prime}$. Como $W_{1}^{\prime} \in \mathcal{L}$, temos que $X$ não é maximal em $\mathcal{L}$.

Seja $(A, B)$ um conceito do qual desejamos listar todos os elementos que são cobertos por ele. As Proposições 6.1 e 6.2 caracterizam as extensões de conceitos cobertos por $(A, B)$, e induzem um algoritmo simples para isto: basta construirmos a família $\mathcal{L}$, e depois descartar elementos não maximais para, finalmente, derivar cada um dos elementos restantes, obtendo o conjunto de conceitos cobertos por $(A, B)$. No entanto, consideraremos um algoritmo ligeiramente diferente, e o chamaremos de EncontraVizinhosDeBaixo: construiremos um conjunto chamado candidatos que armazena todos os conceitos cujas extensões pertencem a $\mathcal{L}$, e depois da construção deste conjunto, faremos o descarte de conceitos que possuem extensão não maximal, dentre todas as extensões de conceitos em candidatos. Tal processo está exposto no Algoritmo 4. A descrição da rotina ElementosMaximais, que realiza as remoções do conjunto candidatos, é trivial e não será explicitada aqui.

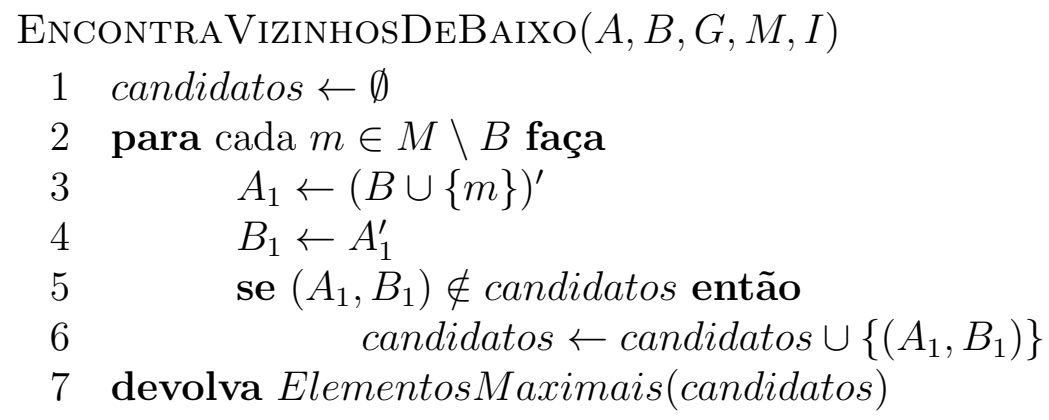

Algoritmo 4: Algoritmo EncontraVizinhosDeBaixo, para a determinação de conceitos cobertos por um determinado conceito $(A, B)$.

O algoritmo Próximos Vizinhos, que se encontra descrito no Algoritmo 5, encontra o conjunto de conceitos $\mathfrak{B}$, bem como a lista de adjacências de cada $(A, B) \in \mathfrak{B}$. A estratégia do algoritmo é encontrar os conceitos por níveis, do menor ao maior ${ }^{1}$. O nível mais alto consiste em $\left\{\left(G, G^{\prime}\right)\right\}$. A partir de um nível arbitrário, obtemos o próximo nível aplicando a função EncontraVizinhosDeBaixo para cada conceito do nível atual.

\footnotetext{
${ }^{1}$ Expomos aqui o algoritmo conforme a apresentação de Carpineto e Romano. A definição deles de adjacência no diagrama de Hasse é dual à nossa: $x$ é coberto por $y$ se e só se existe arco $(y, x)$. Esta estratégia de descoberta, "de cima para baixo", resulta em adjacências simétricas às definidas por nós na subseção 2.5.1.
} 


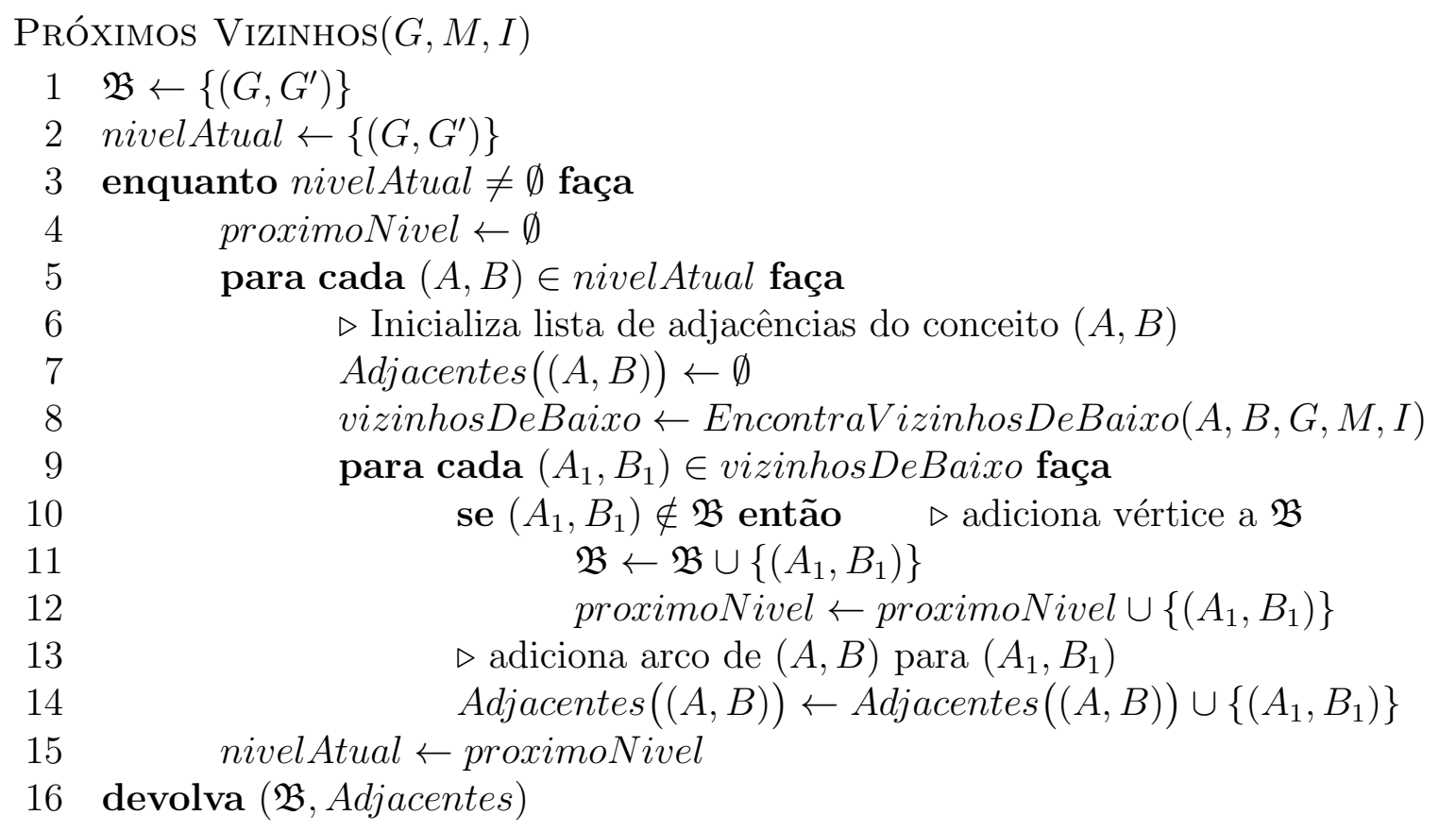

Algoritmo 5: Próximos Vizinhos

\subsubsection{Análise de complexidade}

Analisaremos o tempo consumido pelo algoritmo Próximos Vizinhos em duas etapas, analisando primeiramente a complexidade da subrotina EncontraVizinhosDeBaixo.

Análise de EncontraVizinhosDeBaixo. Consideremos as linhas internas do laço, isto é, linhas de 3 a 6 . A linha 3 pode ser executada em $O(|G|)$, tirando proveito do fato de que $Y^{\prime}=X$ é dado como entrada e $(Y \cup\{m\})^{\prime}=Y^{\prime} \cap\{m\}^{\prime}$, pelo Lema 2.6; ou seja, a linha 3 pode ser implementada calculando a derivação de um atributo e calculando a intersecção de dois subconjuntos de $G$ (ambas operações de complexidade $O(|G|)$ ). A linha 4 pode ser implementada trivialmente com complexidade $O(|G||M|)$. Se utilizarmos uma estrutura de lista para representar o conjunto candidatos, teremos tamanho máximo $O(|M|)$ para tal lista. A linha 5 pode ser implementada comparando a extensão $X_{1}$ com a extensão de cada elemento da lista, (portanto $O(|G|)$ por comparação), resultando numa complexidade de $O(|G||M|)$ por vez que a linha 5 é executada. Utilizando uma lista para representar candidatos, cujos elementos são referências para conceitos $(A, B)$, podemos obter complexidade $O(1)$ por execução da linha 6. O laço de EncontraVizinhosDeBaixo itera $O(|M|)$ vezes, resultando numa complexidade de $O\left(|G||M|^{2}\right)$ para a execução completa do laço.

Resta-nos analisar a complexidade da função ElementosMaximais. Esta função realiza eventuais remoções na lista candidatos, o que motiva o uso de uma lista ligada, para obtermos complexidade de tempo $O(1)$ por remoção de elemento. Então a lista ligada candidatos é passada para a função ElementosMaximais e esta realiza um teste para cada par de elementos $\left(A_{i}, B_{i}\right)$ e $\left(A_{j}, B_{j}\right)$ tais que $j>i$, da seguinte maneira: se $A_{i} \supsetneq A_{j}$, então $\left(A_{j}, B_{j}\right)$ não é coberto por $(A, B)$ e, neste caso, $\left(A_{j}, B_{j}\right)$ é removido da lista. Como este teste de comparação é feito sobre dois subconjuntos de $G$, temos um gasto de $O(|G|)$ unidades de tempo para isto. 
Logo, a complexidade de tempo desta implementação de ElementosMaximais é $O\left(|G||M|^{2}\right)$. Concluímos que a complexidade de tempo de EncontraVizinhosDeBaixo é $O\left(|G||M|^{2}\right)$.

Análise de Próximos Vizinhos. O laço da linha 3 itera exatamente o número de níveis do reticulado, enquanto o laço da linha 5 itera exatamente o número de conceitos no nível atual. Ou seja, as linhas internas ao laço da linha 5 (isto é, linhas 6 a 14) iteram exatamente uma vez por conceito do reticulado ( $|\mathfrak{B}|$ vezes $)$.

Analisamos agora o consumo de tempo das linhas 6 a 14. Já vimos que a linha 8 consome tempo $O\left(|G||M|^{2}\right)$. As linhas internas ao laço da linha 9, isto é, as linhas de 10 a 14, executam $\mid$ vizinhosDeBaixo $\mid=O(|M|)$ vezes. Se usarmos uma árvore de prefixos para representarmos as intenções de $\mathfrak{B}$ e, em cada vértice da árvore de prefixos mantivermos uma referência à extensão associada àquela intenção, então cada execução da linha 10 consumirá tempo $O(|M|)$, conforme o Lema 5.3. Idem para a linha 11. Para a linha 12, proximoNivel pode ser implementado como uma lista ligada, o que significa complexidade $O(1)$ por execução. No momento da execução da linha 14 , o conceito $\left(A_{1}, B_{1}\right)$ certamente está em $\mathfrak{B}$ e podemos supor que temos o vértice da árvore de prefixos que representa $\mathfrak{B}$ associado a $\left(A_{1}, B_{1}\right)$ (seja como subproduto da busca (linha 10), ou da adição (linha 11)). Para adicionarmos o $\operatorname{arco}\left(A_{1}, B_{1}\right) \rightarrow(A, B)$, simplesmente adicionamos uma referência a $\left(A_{1}, B_{1}\right)$ à lista de adjacências Adjacentes $((A, B))$. Então a linha 14 consome $O(1)$ unidades de tempo.

Em suma, a complexidade de tempo da linha 8 domina o tempo gasto pelas linhas 6 a 14, que, durante todo o algoritmo, consomem tempo $O\left(|\mathfrak{B}||M|^{2}\right)$. O consumo total do algoritmo Próximos Vizinhos é portanto $O\left(|\mathfrak{B}||G||M|^{2}\right)$.

\subsection{Algoritmo de Nourine e Raynaud}

Considere um conjunto finito $X$, e seja $\mathcal{F} \subseteq \mathcal{P}(X)$ uma família de subconjuntos de $X$. Por definição, o fecho de $\mathcal{F}$ por uniões é:

$$
\overline{\mathcal{F}}=\left\{\bigcup_{F \in I} F \mid I \subseteq \mathcal{F}\right\} .
$$

Por exemplo, seja $X=\{1,2,3,4,5,6,7,8\}$ e considere a seguinte família:

$$
\mathcal{F}=\{\{2,3,4,5\},\{4,5,6,7,8\},\{2,3,6,7,8\},\{3,4,5,8\}\} .
$$

Para facilitar a notação, se não houver ambiguidade quanto aos elementos de cada subconjunto, omitiremos as vírgulas entre seus elementos. Assim, neste exemplo, escrevere$\operatorname{mos} \mathcal{F}=\{\{2345\},\{45678\},\{23678\},\{3458\}\}$. A fim de ilustrarmos a definição, destacamos que $\{2345678\}$ é um dos subconjuntos presentes em $\overline{\mathcal{F}}$, uma vez que, quando escolhemos $I=\{\{2345\},\{45678\}\} \subseteq \mathcal{F}$, temos $\bigcup_{F \in I} F=\{2345678\}$. Com relação a este exemplo, é fácil, apesar de laborioso, determinar que o fecho de $\mathcal{F}$ por uniões é:

$$
\overline{\mathcal{F}}=\{\{\},\{2345\},\{45678\},\{23678\},\{3458\},\{2345678\},\{23458\},\{345678\}\} .
$$

Como $\overline{\mathcal{F}}$ é um subconjunto de $\mathcal{P}(X)$, a estrutura $\langle\overline{\mathcal{F}}, \subseteq\rangle$ é uma ordem parcial ${ }^{2}$, e podemos representá-la através de um diagrama de Hasse. Na Figura 6.3, se encontra representado

\footnotetext{
${ }^{2}$ É possível mostrar mais: um fecho por uniões é sempre um reticulado.
} 
tal diagrama para o fecho descrito em (6.2), bem como uma rotulação posicionada acima de vértices, que será definida em seguida.

Definimos a função $\gamma: \overline{\mathcal{F}} \rightarrow \mathcal{P}(\mathcal{F})$, como

$$
\gamma(G)=\{F \in \mathcal{F} \mid F \subseteq G\}
$$

Isto é, $\gamma$ leva um elemento $G$ do fecho por uniões na coleção dos subconjuntos da família $\mathcal{F}$ contidos em $G$. Esta função será muito importante para o algoritmo a ser apresentado.

Ilustraremos os valores da função $\gamma$ no caso do fecho $\overline{\mathcal{F}}$ em (6.2). A fim de obtermos maior clareza para esta ilustração, associaremos um símbolo a cada subconjunto da família $\mathcal{F}$ em (6.1): $F_{1}=\{2345\} ; F_{2}=\{45678\} ; F_{3}=\{23678\} ; F_{4}=\{3458\}$. Na Figura 6.3, os valores da função $\gamma$ estão representados acima dos vértices do diagrama de Hasse.

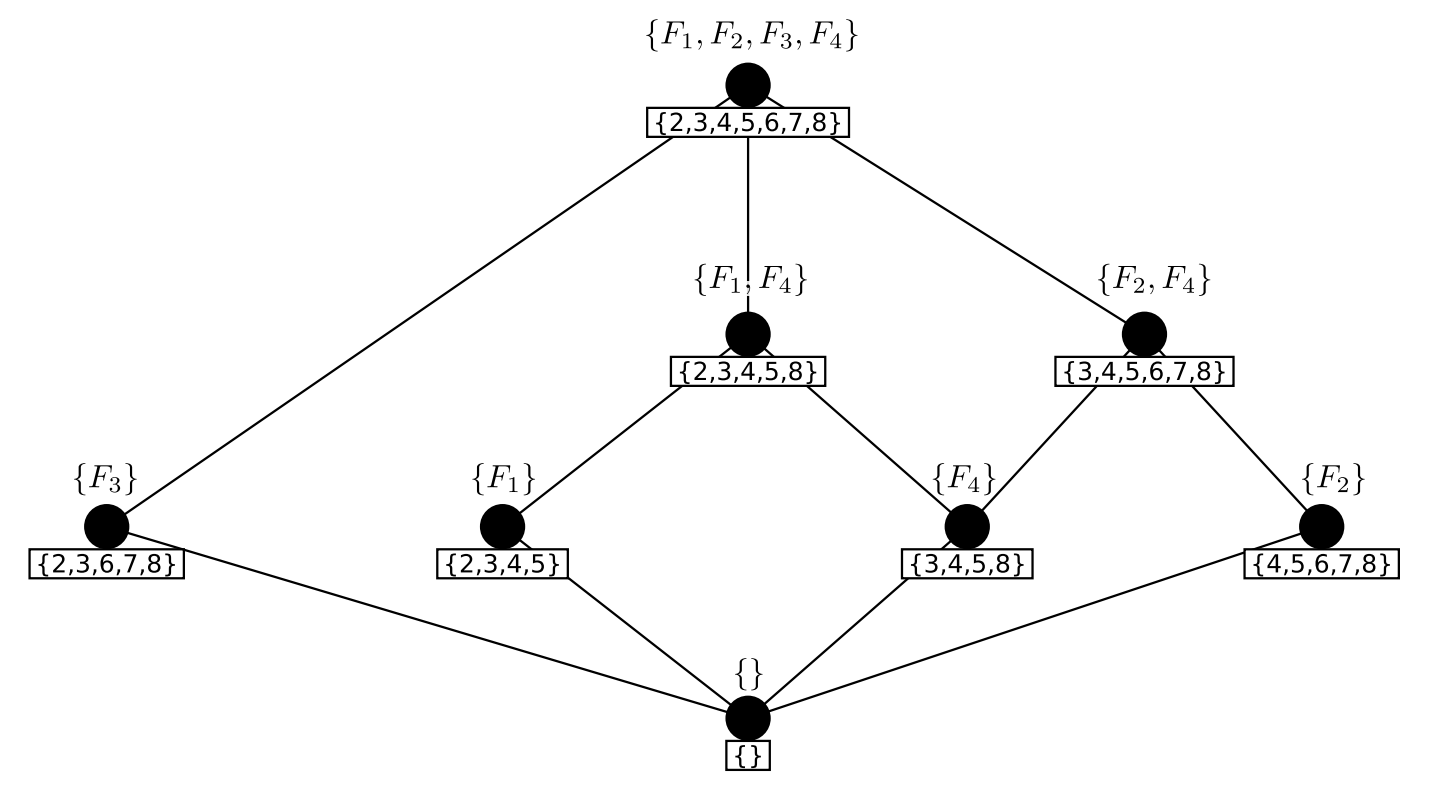

Figura 6.3: Diagrama de Hasse do fecho por uniões (6.2), juntamente com os valores da função $\gamma$.

Consideraremos o seguinte problema:

Reticulado do fecho por uniões de uma família (RFUF): Dado um conjunto finito $X$ e uma família $\mathcal{F} \subseteq \mathcal{P}(X)$, encontrar o diagrama de Hasse de $\langle\overline{\mathcal{F}}, \subseteq\rangle$.

Nourine e Raynaud mostraram um algoritmo $O((|X|+|\mathcal{F}|) \cdot|\mathcal{F}| \cdot|\overline{\mathcal{F}}|)$ para o RFUF [NR99], assim como um argumento, baseado na lei de de Morgan, que estabelece uma redução do problema da determinação do reticulado de conceitos para o RFUF. Dado um contexto $\mathbb{K}=(G, M, I)$, a complexidade de tempo total desta solução é $O(|\mathfrak{B}(\mathbb{K})| \cdot|G| \cdot|M|)$.

$\mathrm{O}$ algoritmo de Nourine e Raynaud pode ser considerado como um processo de duas etapas: primeiramente constrói-se uma lista com os elementos de $\overline{\mathcal{F}}$, assim como uma estrutura auxiliar chamada árvore lexicográfica de $\overline{\mathcal{F}}$. Tal estrutura contém toda a informação sobre quais elementos pertencem a $\overline{\mathcal{F}}$ e quais são os valores de $\gamma$, mas não descreve explicitamente 
a cobertura entre elementos de $\overline{\mathcal{F}}$, ou seja, não contém de maneira explícita a informação sobre o diagrama de Hasse de $\langle\overline{\mathcal{F}}, \subseteq\rangle$. Na segunda etapa, são realizadas contagens sobre tal estrutura auxiliar construída durante a primeira etapa, obtendo assim toda a informação sobre cobertura dos elementos de $\overline{\mathcal{F}}$.

Para simplificar a descrição do algoritmo a ser exposto, supomos sempre que $\emptyset \notin \mathcal{F}$, uma vez que a inclusão de $\emptyset$ em $\mathcal{F}$ não muda seu fecho por uniões.

Seja $\mathcal{F}=\left\{F_{1}, F_{2}, \ldots, F_{k}\right\} \subseteq \mathcal{P}(X)$. O primeiro algoritmo que mostraremos efetua a construção do fecho $\overline{\mathcal{F}}$, através da construção do fecho de subfamílias $\mathcal{F}_{i}=\left\{F_{1}, F_{2}, \ldots, F_{i}\right\}$, com $i$ crescendo de 1 até $k$. Note que $\mathcal{F}_{0}=\emptyset, \overline{\mathcal{F}_{0}}=\{\emptyset\}$ e $\overline{\mathcal{F}_{k}}=\overline{\mathcal{F}}$. De forma geral:

$$
\overline{\mathcal{F}_{i}}=\left\{\bigcup_{F \in S} F \mid S \subseteq\left\{F_{1}, \ldots, F_{i}\right\}\right\}
$$

O Algoritmo 6 descreve a construção de $\overline{\mathcal{F}}=\overline{\mathcal{F}_{k}}$, através de uma construção incremental de cada um dos fechos parciais $\overline{\mathcal{F}}_{i}$. (Por abuso de linguagem, permitiremos nominar variáveis $\overline{\mathcal{F}_{i}}$.)

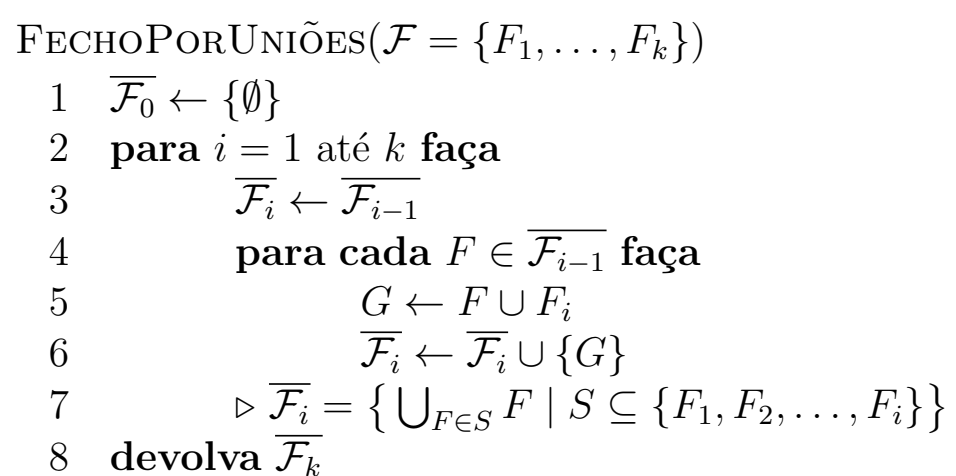

Algoritmo 6: Construção do fecho por uniões.

O algoritmo acima é essencialmente uma versão dual do Algoritmo 1, que encontra o fecho por intersecções de intenções-objetos. De fato, a correção do Algoritmo 6 pode ser provada facilmente por indução, assim como naquela ocasião. Assumimos, assim, o resultado abaixo, sem necessidade de demonstração.

Proposição 6.3. Após cada iteração do laço externo do Algoritmo 6, o valor da variável $\overline{\mathcal{F}_{i}}$ é o fecho parcial $\left\{\bigcup_{F \in S} F \mid S \subseteq\left\{F_{1}, F_{2}, \ldots, F_{i}\right\}\right\}$. Em particular, o Algoritmo 6 devolve corretamente o valor de $\overline{\mathcal{F}}$.

A primeira fase do algoritmo de Nourine e Raynaud, no entanto, não necessita apenas do valor de $\overline{\mathcal{F}}$. Também se fazem necessários os valores da função $\gamma$. O algoritmo que encontra os valores de $\gamma$, além de $\overline{\mathcal{F}}$, é uma pequena variação do Algoritmo 6, e se encontra descrito no Algoritmo 7. (Por abuso de linguagem, permitiremos nominar variáveis $\gamma_{i}$.) 


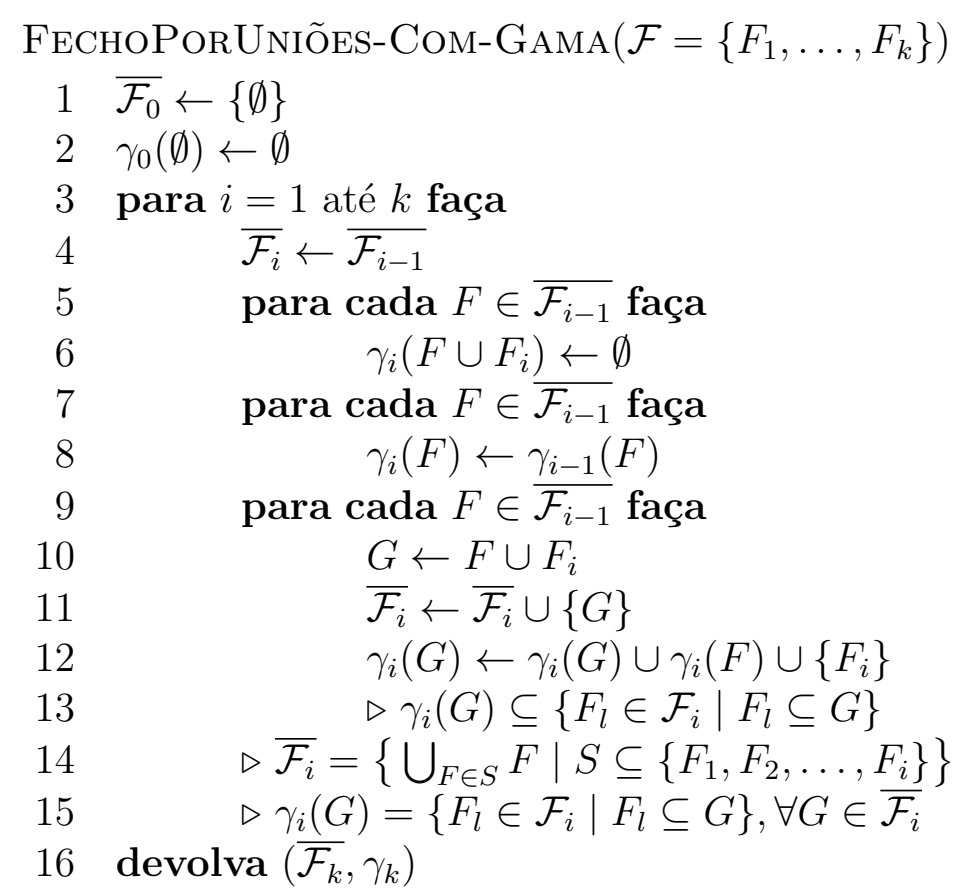

Algoritmo 7: Construção do fecho por uniões e da função $\gamma$.

Os invariantes das linhas 14 e 15 valem como consequência da próxima proposição, assim como da Proposição 6.3.

Proposição 6.4. Após cada iteração do laço externo do Algoritmo \%, o valor da variável $\gamma_{i}(G)$ é $\left\{F_{l} \in \mathcal{F}_{i} \mid F_{l} \subseteq G\right\}$, para todo $G \in \overline{\mathcal{F}}_{i}$. Em particular, o Algoritmo 7 devolve corretamente o valor de $\gamma$.

Prova. Para $1 \leq i \leq k$ e $1 \leq j \leq\left|\overline{\mathcal{F}_{i-1}}\right|$, sejam $F^{i, j}$ e $G^{i, j}$ os valores das variáveis $F$ e $G$, respectivamente, depois da $j$-ésima execução da linha 10, durante a $i$-ésima execução do laço da linha 3. Vamos mostrar que vale o invariante da linha 13, isto é, provaremos que para todo $i, j$ satisfazendo $1 \leq i \leq k$ e $1 \leq j \leq\left|\overline{\mathcal{F}_{i-1}}\right|$, após a execução da linha 12 , vale a seguinte inclusão:

$$
\gamma_{i}\left(G^{i, j}\right) \subseteq\left\{F_{l} \in \mathcal{F}_{i} \mid F_{l} \subseteq G^{i, j}\right\} .
$$

Para isso, faremos indução dupla em $i$ e $j$. Para o caso base, vamos mostrar que (6.3) vale para $i=1$ e todo $1 \leq j \leq\left|\overline{\mathcal{F}_{0}}\right|$. Como $\left|\overline{\mathcal{F}_{0}}\right|=1$, isto significa mostrar a validade de (6.3) para $i=1$ e $j=1$. É fácil verificar que $G^{1,1}=F_{1}$ e $\gamma_{1}\left(G^{1,1}\right)=\left\{F_{1}\right\}=\left\{F_{l} \in \mathcal{F}_{1} \mid F_{l} \subseteq G^{1,1}\right\}$.

Agora, seja $i^{*} \geq 2$. Suponha que (6.3) vale para todo $i \leq i^{*}-1$ e todo $j \leq\left|\overline{\mathcal{F}_{i-1}}\right|$. Mostraremos que (6.3) vale para $i=i^{*}$ e todo $j \leq\left|\overline{\mathcal{F}_{i^{*}-1}}\right|$. Para isto, faremos indução completa em $j$. Isto é, mostraremos que, para todo $j^{*}$, se (6.3) valer para $i=i^{*}$ e todo $j<j^{*}$, então (6.3) vale para $i=i^{*}$ e $j=j^{*}$. Seja $j^{*}$ com $1 \leq j^{*} \leq\left|\overline{\mathcal{F}_{i^{*}-1}}\right|$. Ao juntarmos as duas hipóteses de indução, temos a seguinte inclusão:

$$
\gamma_{i}\left(G^{i, j}\right) \subseteq\left\{F_{l} \in \mathcal{F}_{i} \mid F_{l} \subseteq G^{i, j}\right\}, \quad \forall i, j: 1 \leq i<i^{*}, 1 \leq j \leq\left|\overline{\mathcal{F}_{i-1}}\right| \text { ou } i=i^{*}, 1 \leq j<j^{*} .
$$

Isto é, temos que (6.3) vale para todo par $(i, j)$ lexicograficamente menor que $\left(i^{*}, j^{*}\right)$. Observe que, na linha 12, quando $i=i^{*}$ e $j=j^{*}$, temos que a variável $\gamma_{i^{*}}\left(G^{i^{*}}, j^{*}\right)$ recebe o valor 
$\gamma_{i^{*}}\left(G^{i^{*}, j^{*}}\right) \cup \gamma_{i^{*}}\left(F^{i^{*}, j^{*}}\right) \cup\left\{F_{i^{*}}\right\}$. Vamos mostrar que cada um dos três conjuntos de tal união é subconjunto de $\left\{F_{l} \in \mathcal{F}_{i^{*}} \mid F_{l} \subseteq G^{i^{*}}, j^{*}\right\}$, e assim, a tese seguirá. Inicialmente, mostraremos tal afirmação para o conjunto $\gamma_{i^{*}}\left(G^{i^{*}, j^{*}}\right)$. Separaremos em dois casos:

i) Suponha que não exista um par $(\hat{i}, \hat{j})$ lexicograficamente menor que $\left(i^{*}, j^{*}\right)$ satisfazendo $G^{i^{*}, j^{*}}=G^{\hat{i}, \hat{j}}$. Neste caso, para qualquer iteração do laço externo, digamos, a $m$-ésima, com $m \leq i^{*}$, o valor de $\gamma_{m}\left(G^{i^{*}, j^{*}}\right)$ é atualizado apenas nas linhas 6 e 8. Assim, temos que $\gamma_{i^{*}}\left(G^{i^{*}, j^{*}}\right)=\emptyset$, e a afirmação segue.

ii) Suponha que exista um par $(\hat{i}, \hat{j})$ lexicograficamente menor que $\left(i^{*}, j^{*}\right)$ satisfazendo $G^{i^{*}, j^{*}}=G^{\hat{i}, \hat{j}}$. Neste caso, suponha que $(\hat{i}, \hat{j})$ é o maior par, lexicograficamente, com essa propriedade. Assim, claramente o valor de $\gamma_{i^{*}}\left(G^{i^{*}, j^{*}}\right)$, antes da execução da linha 12, é igual a $\gamma_{\hat{i}}\left(G^{\hat{i}, \hat{j}}\right)$. Pela hipótese de indução, temos que:

$$
\begin{aligned}
\gamma_{\hat{i}}\left(G^{\hat{i}, \hat{j}}\right) & \subseteq\left\{F_{l} \in \mathcal{F}_{\hat{i}} \mid F_{l} \subseteq G^{\hat{i}, \hat{j}}\right\} \\
& =\left\{F_{l} \in \mathcal{F}_{\hat{i}} \mid F_{l} \subseteq G^{i^{*}, j^{*}}\right\} \subseteq\left\{F_{l} \in \mathcal{F}_{i^{*}} \mid F_{l} \subseteq G^{i^{*}, j^{*}}\right\} .
\end{aligned}
$$

Portanto, temos que, antes da execução da linha 12, vale que $\gamma_{i^{*}}\left(G^{i^{*}, j^{*}}\right)$ é um subconjunto de $\left\{F_{l} \in \mathcal{F}_{i^{*}} \mid F_{l} \subseteq G^{i^{*}, j^{*}}\right\}$. Um argumento idêntico ao acima mostra que $\gamma_{i^{*}}\left(F^{i^{*}, j^{*}}\right)$ é um subconjunto de $\left\{F_{l} \in \mathcal{F}_{i^{*}} \mid F_{l} \subseteq F^{i^{*}, j^{*}}\right\}$. A atribuição presente na linha 10 implica que $F^{i^{*}, j^{*}} \subseteq G^{i^{*}, j^{*}}$, e portanto, $\gamma_{i^{*}}\left(F^{i^{*}, j^{*}}\right)$ é um subconjunto de $\left\{F_{l} \in \mathcal{F}_{i^{*}} \mid F_{l} \subseteq G^{i^{*}, j^{*}}\right\}$. A inclusão $\left\{F_{i^{*}}\right\} \subseteq\left\{F_{l} \in \mathcal{F}_{i^{*}} \mid F_{l} \subseteq G^{i^{*}, j^{*}}\right\}$ segue pelo fato de que $F_{i^{*}} \subseteq G^{i^{*}, j^{*}}$, também por causa da atribuição da linha 10. Assim, mostramos as três inclusões mencionadas e a tese segue.

Agora mostraremos o invariante da linha 15:

$$
\gamma_{i}(G)=\left\{F_{l} \in \mathcal{F}_{i} \mid F_{l} \subseteq G\right\} \text {, para todo } G \in \overline{\mathcal{F}}_{i} .
$$

Após a primeira iteração do laço da linha 3 , é fácil verificar que $i=1, \overline{\mathcal{F}_{1}}=\left\{\emptyset, F_{1}\right\}$ e que $\gamma_{1}(\emptyset)=\emptyset$ e $\gamma_{1}\left(F_{1}\right)=\left\{F_{1}\right\}$. Para o passo de indução, seja $i \geq 2$, e suponha que vale que $\gamma_{i-1}(G)=\left\{F_{l} \in \mathcal{F}_{i-1} \mid F_{l} \subseteq G\right\}$, para todo $G \in \overline{\mathcal{F}_{i-1}}$. Seja $G \in \overline{\mathcal{F}}_{i}$. Vamos mostrar que, após a $i$-ésima execução do laço da linha 3 , temos que $\gamma_{i}(G)=\left\{F_{l} \in \mathcal{F}_{i} \mid F_{l} \subseteq G\right\}$. Dividiremos em dois casos:

i) Suponha que $F_{i} \not \subseteq G$ : Neste caso, temos que o valor de $F \cup\left\{F_{i}\right\}$ presente na linha 10, em nenhuma iteração do laço da linha 9, é igual a $G$. Logo, o valor de $\gamma_{i}(G)$, após ser inicializado na linha 8 , é mantido durante toda a iésima iteração. Ou seja, segue que $\gamma_{i}(G)=\gamma_{i-1}(G)$. Além disso, pela hipótese de indução, podemos concluir que $\gamma_{i-1}(G)=\left\{F_{l} \in \mathcal{F}_{i-1} \mid F_{l} \subseteq G\right\}=\left\{F_{l} \in \mathcal{F}_{i} \mid F_{l} \subseteq G\right\}$.

ii) Suponha que $F_{i} \subseteq G$ : Neste caso, seja $H=\bigcup\left\{F_{l} \in \mathcal{F}_{i-1} \mid F_{l} \subseteq G\right\}$. Note que $H \in \overline{\mathcal{F}_{i-1}}$ e que $G=H \cup\left\{F_{i}\right\}$, e, consequentemente, temos a seguinte igualdade: $\left\{F_{l} \in \mathcal{F}_{i-1} \mid F_{l} \subseteq H\right\}=\left\{F_{l} \in \mathcal{F}_{i-1} \mid F_{l} \subseteq G\right\}$. Em alguma iteração do laço da linha 9, temos que $F=H$, e assim, em tal iteração, o valor de $\gamma_{i}(H) \cup\left\{F_{i}\right\}$ é incluído em $\gamma_{i}(G)$. Pela inicialização de $\gamma_{i}(H)$ vale que $\gamma_{i}(H) \supseteq \gamma_{i-1}(H)$ e, pela hipótese de indução, temos necessariamente que $\gamma_{i-1}(H)=\left\{F_{l} \in \mathcal{F}_{i-1} \mid F_{l} \subseteq H\right\}=\left\{F_{l} \in \mathcal{F}_{i-1} \mid F_{l} \subseteq G\right\}$. Assim, 
vale que, durante tal iteração do laço da linha 9, após a execução da linha 12, temos que $\gamma_{i}(G) \supseteq\left\{F_{l} \in \mathcal{F}_{i-1} \mid F_{l} \subseteq G\right\} \cup\left\{F_{i}\right\}=\left\{F_{l} \in \mathcal{F}_{i} \mid F_{l} \subseteq G\right\}$. A inclusão contrária segue ao escolhermos o maior $j$ tal que $G^{i, j}=G$ e aplicarmos (6.3).

A primeira etapa do algoritmo tratado nesta seção é essencialmente uma versão do Algoritmo 7, diferindo apenas na utilização da estrutura de dados auxiliar que mencionamos, com a finalidade de obter-se uma complexidade computacional satisfatória para a execução das linhas 11 e 12. De fato, uma implementação ingênua da linha 11, como por exemplo, uma implementação que verifique a presença ou ausência de $G$ em uma lista ligada representando $\overline{\mathcal{F}_{i}}$, acarreta uma complexidade total em $\Omega\left(|\overline{\mathcal{F}}|^{2}\right)$ para o Algoritmo 7 , o que inviabilizaria uma solução do RFUF com a complexidade almejada.

No que segue, introduziremos qual será tal estrutura auxiliar e, em seguida, explicitaremos a quais operações desejamos submeter tal estrutura, para enfim detalharmos como a representação de tal estrutura é feita em uma implementação do algoritmo, e qual é a complexidade computacional que pode ser obtida por cada operação.

\subsection{1 Árvore Lexicográfica}

Suponha dado um conjunto finito $X$, uma família de subconjuntos $\mathcal{F} \subseteq \mathcal{P}(X)$, e uma ordem total estrita sobre o conjunto $X$, que denotamos por $<$.

Definições Uma arborescência é um grafo orientado $(V, A)$, obtido após orientar-se as arestas de uma árvore enraizada no sentido do afastamento de sua raiz $r$. O vértice $r$ é dito raiz da arborescência, e por vezes utilizamos o termo arborescência de raiz $r$ para nos referirmos à tripla $(V, A, r)$. Em uma arborescência, um vértice é dito folha se possuir grau de entrada igual a um e grau de saída igual a zero.

Seja $\mathcal{T}=(V, A, r)$ uma arborescência de raiz $r$. Se $\lambda: A \rightarrow X$ for uma função, associando um elemento de $X$ para cada arco de $\mathcal{T}$, dizemos que $\lambda$ é uma rotulação de arcos. Dada uma rotulação de arcos $\lambda$, chamamos de rotulação de vértices induzida por $\lambda$, e denotamos por $\lambda^{V}$ a função que, para um vértice $v \in V$ associa a união dos rótulos no único caminho de $r$ até $v$ em $\mathcal{T}$. Note que já definimos, durante a apresentação da árvore de prefixos, no Capítulo 5, uma rotulação de vértices, mas que não deve ser confundida com esta.

Dada uma família $\mathcal{F}$ de subconjuntos de $X$, a árvore lexicográfica $\mathcal{T}_{\mathcal{F}}$ é uma quádrupla $(V, A, r, \lambda)$, onde $(V, A, r)$ é uma arborescência de raiz $r$ e $\lambda$ é uma rotulação dos seus arcos tal que:

1. Se $u, v, z \in V \operatorname{com} u v, v z \in A$ então $\lambda(u v)<\lambda(v z)$.

2. Se $x, y, y^{\prime} \in V \operatorname{com} x y, x y^{\prime} \in A$ e $y \neq y^{\prime}$, então $\lambda(x y) \neq \lambda\left(x y^{\prime}\right)$.

3. A rotulação $\lambda^{V}: V \rightarrow \mathcal{P}(X)$ induzida por $\lambda$ é tal que, para todo $F \in \mathcal{F}$, existe $v_{F} \in V$ tal que $\lambda\left(v_{F}\right)=F$.

4. Se $v \in V$ é folha, então $\lambda(v) \in \mathcal{F}$. 
Observe que as condições 1 e 2 implicam a injetividade de $\lambda^{V}$. Portanto, o vértice $v_{F}$ presente na condição 3 é único, e assim, o conjunto $\mathcal{F}$ está em bijeção com o conjunto $\{v \in V \mid \lambda(v) \in \mathcal{F}\}$. Este conjunto é chamado de conjunto de vértices finais. Também convém observar que a definição de árvore lexicográfica não exige que a família $\mathcal{F}$ seja o fecho por uniões de alguma família.

A árvore lexicográfica da família (6.2) está representada na Figura 6.4, onde convencionamos que as orientações dos arcos devem ser subentendidas como partindo de um vértice com maior $Y$-coordenada para um vértice com menor $Y$-coordenada. Ademais, os vértices finais de uma árvore lexicográfica são representados possuindo um círculo adicional.

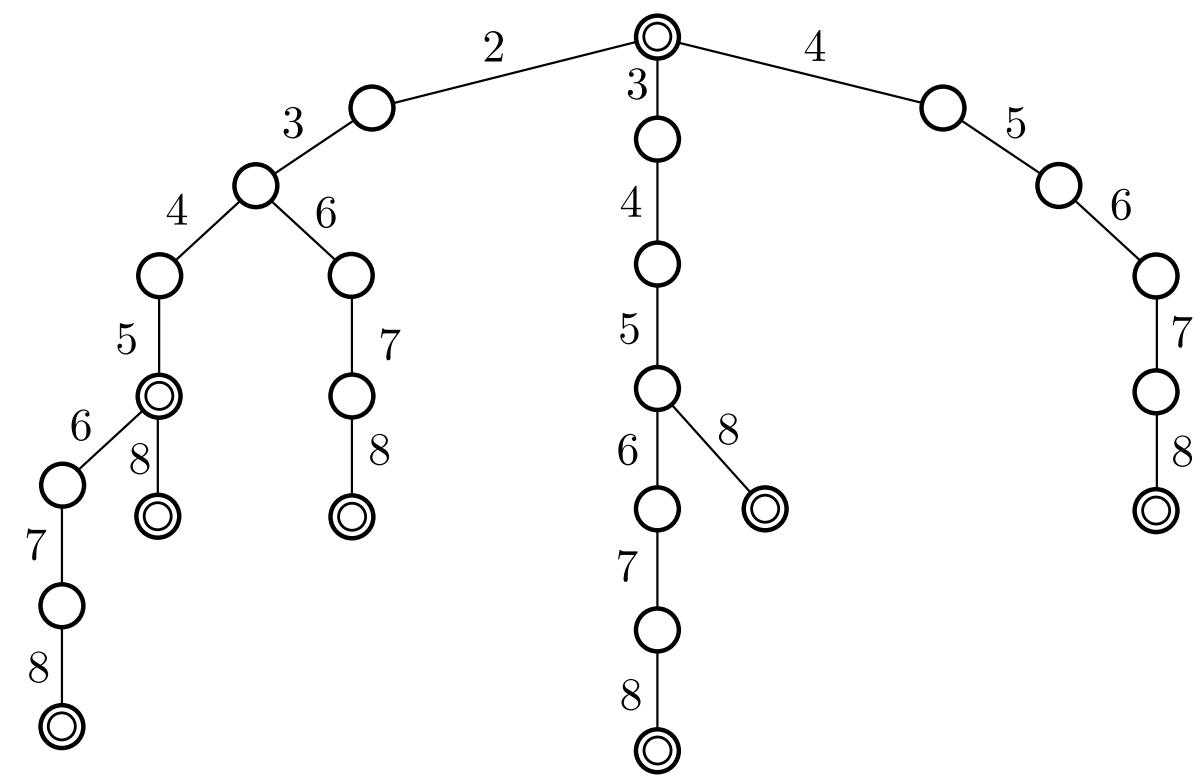

Figura 6.4: Árvore lexicográfica do fecho por uniões $\overline{\mathcal{F}}$ descrito em (6.2).

No caso de $\mathcal{T}_{\overline{\mathcal{F}}}$ ser a árvore lexicográfica de um fecho por uniões $\overline{\mathcal{F}}$, podemos representar sob os vértices finais de $\mathcal{T}_{\overline{\mathcal{F}}}$ o valor da função $\gamma$. Um exemplo está presente na Figura 6.5, onde mantivemos a associação entre letras e subconjuntos de $\mathcal{F}$ utilizada na Figura 6.3. Observe que, como $\overline{\mathcal{F}}$ é um fecho por uniões, então $\emptyset \in \overline{\mathcal{F}}$, e seu vértice final associado é a raiz da árvore lexicográfica. Ademais, o valor de $\gamma(\emptyset)$ é vazio, uma vez que supomos que $\emptyset \notin \mathcal{F}$.

\subsubsection{Estrutura utilizada para representação da árvore lexicográfica}

Durante a primeira etapa do algoritmo, a estrutura de árvore lexicográfica será utilizada para representar os conjuntos de $\overline{\mathcal{F}}$ já presentes no fecho $\mathcal{G}=\overline{\mathcal{F}_{i}}$ sendo construído. Na segunda etapa, será importante a presença, na árvore lexicográfica, da informação sobre a cardinalidade de $\gamma(F)$, para cada $F \in \overline{\mathcal{F}}$. Mais especificamente, ao longo das duas etapas, precisamos realizar as seguintes operações com satisfatória complexidade computacional:

1) $\operatorname{Pertence}\left(G, \mathcal{T}_{\mathcal{G}}\right)$ : Decide se $G$ está representado em $\mathcal{T}_{\mathcal{G}}$. 


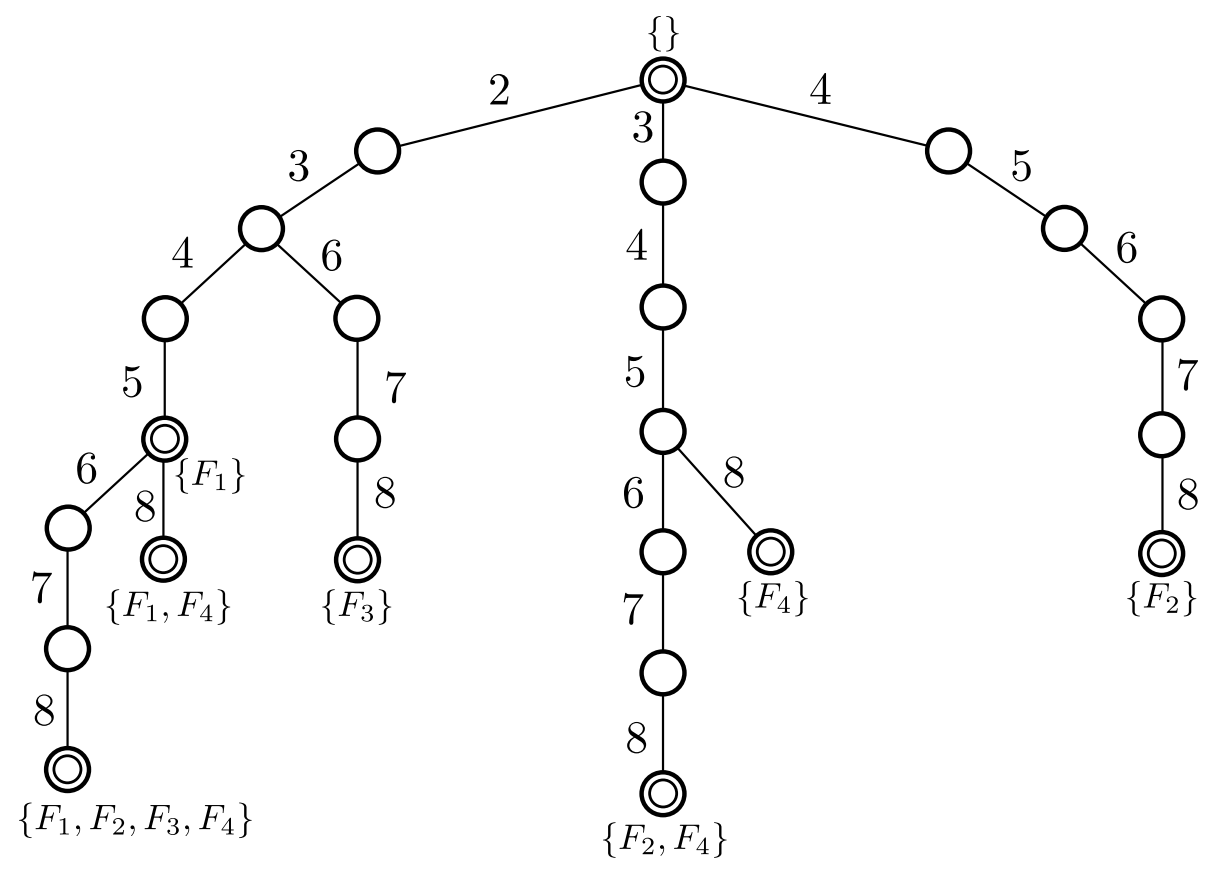

Figura 6.5: Árvore lexicográfica da familia $\overline{\mathcal{F}}$, descrita em (6.2), incluindo os valores de $\gamma$.

2) $\operatorname{Adiciona}\left(G, \mathcal{T}_{\mathcal{G}}\right):$ Adiciona $G$ à $\mathcal{T}_{\mathcal{G}}$.

3) $\operatorname{AtualizaGama}\left(G, F, i, \mathcal{T}_{\mathcal{G}}\right)$ : Atualiza a família $\gamma(G)$ para $\gamma(G) \cup \gamma(F) \cup\left\{F_{i}\right\}$.

4) $\operatorname{CardGama}\left(G, \mathcal{T}_{\mathcal{G}}\right)$ : Devolve a cardinalidade de $\gamma(G)$.

Para tanto, as adjacências de cada vértice da árvore lexicográfica serão representadas por listas ligadas ordenadas segundo os rótulos das arestas. Isto é, se $u$ for um vértice de $\mathcal{T}_{\mathcal{G}}$ cujo conjunto de vértices adjacentes é $S \subseteq V\left(\mathcal{T}_{\mathcal{G}}\right)$, então as adjacências de $v$ são representadas em uma lista ligada, na ordem $\left\{v_{1}, \ldots, v_{k}\right\}=S$ tal que $\lambda\left(u v_{i}\right)<\lambda\left(u v_{j}\right)$ se $i<j$. Para a representaç̧̃a de $\gamma$, mantemos em cada vértice final $v_{F}$ da árvore lexicográfica um vetor binário de $|\mathcal{F}|$ posições. O valor '1' presente na $i$-ésima posição deste vetor indica a presença de $F_{i}$ em $\gamma(F)$, enquanto que o valor '0' indica ausência. A presença do vetor armazenando, desta maneira, o valor de $\gamma$, também identifica que o vértice em questão é um vértice final: vértices não finais não possuem um tal vetor associado.

Lema 6.5. Com a representação da estrutura acima descrita, a operação PERTENCE pode ser implementada para consumir tempo $O(|X|)$, e as operações Adiciona, AtualizaGama $e$ CARDGama para consumirem tempo $O(|X|+|\mathcal{F}|)$.

Prova. Considere a operação $\operatorname{Pertence}\left(G, \mathcal{T}_{\overline{\mathcal{F}}}\right)$, e sejam $x_{1}, \ldots, x_{k}$ os elementos de $G$ conforme a ordem total estrita sobre $X$. Seja, ainda, $r$ a raiz de $\mathcal{T}_{\mathcal{F}}$, e considere o seguinte processo de busca em tal árvore: inicialmente o vértice visitado é $r$. Verifica-se na lista ordenada de adjacências de $r$ se tal vértice é adjacente a algum $v$ com $\lambda(r v)=x_{1}$. Caso negativo, então claramente não há vértice final cujo rótulo é $G$. Caso afirmativo, a busca 
descende para $v$ e verifica se $v$ é adjacente a $w \operatorname{com} \lambda(v w)=x_{2}$, e assim por diante. A busca termina com uma resposta positiva se e somente se é encontrado um vértice final $v$ com $\lambda(v)=G$. Note que, pela propriedade 1 da definição de árvore lexicográfica, no máximo $|X|$ arestas são verificadas em tal busca pelas adjacências.

A operação $\operatorname{Adiciona}\left(G, \mathcal{T}_{\mathcal{G}}\right)$ supõe que $G$ ainda não se encontra representado em $\mathcal{T}_{\mathcal{G}}$ e inicia-se com uma busca idêntica à realizada pela operação PERTENCE, até ocorrer uma de duas coisas:

1. Não existe vértice $v \in V\left(\mathcal{T}_{\mathcal{G}}\right)$ tal que $\lambda(v)=G$,

2. Existe vértice $v \in V\left(\mathcal{T}_{\mathcal{G}}\right)$ tal que $\lambda(v)=G$, mas $v$ não é final.

No primeiro caso, são criados não mais do que $|X|$ vértices, sendo um deles um vértice final, assim como não mais do que $|X|$ arestas em $\mathcal{T}_{\mathcal{G}}$, resultando na árvore lexicográfica de $\mathcal{G} \cup\{G\}$. O vetor de comprimento $|\mathcal{F}|$, que armazena a informação sobre $\gamma(G)$, também é inicializado para o novo vértice final. No segundo caso, basta alocar tal vetor.

A operação AtualizaGama $\left(G, F, i, \mathcal{T}_{\mathcal{G}}\right)$ pode ser implementada da seguinte maneira: são encontrados os vértices associados a $G$ e $F$ em $\mathcal{T}_{\mathcal{G}}$ conforme a operação PERTENCE recém detalhada. A partir disso, atribui-se 1 para a $i$-ésima posição do vetor que representa $\gamma(G)$, a fim de representar que $F_{i} \in \gamma(G)$. Depois, itera-se sobre o vetor binário $\gamma(F)$, e atribui-se 1 para as posições de $\gamma(G)$ tais que a correspondente posição de $\gamma(F)$ também possua o valor 1 .

Com relação à operação CARDGAma $\left(G, \mathcal{T}_{\overline{\mathcal{F}}}\right)$, podemos realizá-la encontrando o vértice associado a $G$, em tempo $O(|X|)$ e, em seguida, calculando o número de posições com valor igual a 1 do vetor que representa $\gamma(G)$.

Além das operações descritas no Lema 6.5, precisaremos da operação ARVORETRIVIAL, que cria uma árvore lexicográfica somente com um vértice final representando sua raiz. $\mathrm{O}$ algoritmo que constitui a primeira etapa está descrito no Algoritmo 8.

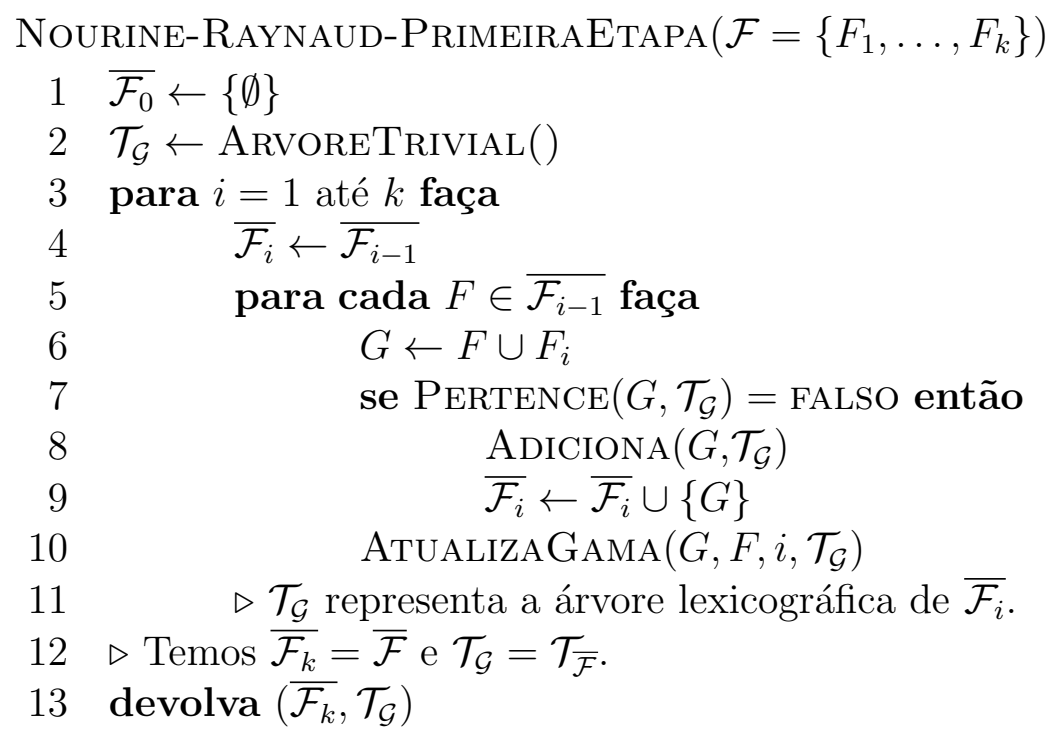

Algoritmo 8: Primeira etapa do algoritmo de Nourine e Raynaud. 
Lema 6.6. O Algoritmo 8 pode ser implementado de maneira a apresentar complexidade de tempo $O(|\overline{\mathcal{F}}| \cdot|\mathcal{F}| \cdot(|X|+|\mathcal{F}|))$ e consumir $O(\overline{\mathcal{F}} \cdot(|X|+|\mathcal{F}|))$ unidades de espaço.

Prova. A afirmação sobre a complexidade de tempo segue diretamente do Lema 6.5. Em termos de espaço, basta observarmos que o número de folhas de $\mathcal{T}_{\overline{\mathcal{F}}}$ é sempre menor ou igual a $\overline{\mathcal{F}}$, e a profundidade de toda folha é limitada superiormente por $|X|$. Logo, há $O(|\overline{\mathcal{F}}| \cdot|X|)$ vértices em $\mathcal{T}_{\overline{\mathcal{F}}}$, e portanto tal quantidade de espaço é suficiente para representarmos os conjuntos de vértices e arcos de $\mathcal{T}_{\overline{\mathcal{F}}}$ utilizando a representação mencionada no Lema 6.5. Falta, no entanto, contabilizar o espaço utilizado para a representação dos valores de $\gamma$. Como $\gamma$ está definido apenas para vértices finais, uma quantidade $O(|\overline{\mathcal{F}}| \cdot|\mathcal{F}|)$ de espaço é suficiente para a representação de todos os valores de $\gamma$ em $\mathcal{T}_{\overline{\mathcal{F}}}$.

\subsubsection{Diagrama de Hasse de $\langle\overline{\mathcal{F}}, \subseteq\rangle$}

Veremos como a construção da árvore lexicográfica de um fecho por uniões $\overline{\mathcal{F}}$ pode auxiliar a obtenção do seu diagrama de Hasse. A função $\gamma$ será particularmente útil para isto.

A seguinte proposição é de imediata demonstração.

Proposição 6.7. Sejam $G, H \in \overline{\mathcal{F}}$ satisfazendo $G \subseteq H$. Então:

$$
H=G \cup \bigcup\{F \in \mathcal{F} \mid F \in \gamma(H) \backslash \gamma(G)\}
$$

O próximo teorema é uma caracterização da cobertura de dois subconjuntos que pertencem ao fecho $\overline{\mathcal{F}}$. Lembramos que se um elemento $G$ for coberto por um elemento $H$, frequentemente expressamos este fato através da notação $G \prec H$.

Teorema 6.8. Sejam $G, H \in \overline{\mathcal{F}}$ com $G \subsetneq H$. As seguintes afirmações são equivalentes:

i) $G$ é coberto por $H$,

ii) Para todo $F_{1}, F_{2} \in \gamma(H) \backslash \gamma(G)$, vale que $F_{1} \backslash G=F_{2} \backslash G$,

iii) Para todo $F \in \gamma(H) \backslash \gamma(G)$, vale que $H=G \cup F$.

Prova. Vamos mostrar as implicações $i i i) \Rightarrow$ ii), ii) $\Rightarrow$ i) e i) $\Rightarrow$ iii).

iii) $\Rightarrow$ ii) : Sejam $F_{1}, F_{2} \in \gamma(H) \backslash \gamma(G)$. Por iii), temos: $H=G \cup F_{1}$ e $H=G \cup F_{2}$. Portanto, $H \backslash G=F_{1} \backslash G=F_{2} \backslash G$.

ii) $\Rightarrow$ i) : Suponha ii) e seja $I \in \overline{\mathcal{F}}$ tal que $G \subsetneq I \subseteq H$. Para mostrarmos que $G \prec H$, basta que provemos $I=H$. Tendo $G \subsetneq I \subseteq H$, podemos escrever

$$
\left\{\begin{array}{c}
H=G \cup \bigcup\{F \in \mathcal{F} \mid F \in \gamma(H) \backslash \gamma(G)\} \\
I=G \cup \bigcup\{F \in \mathcal{F} \mid F \in \gamma(I) \backslash \gamma(G)\}
\end{array}\right.
$$

assim como

$$
\left\{\begin{array}{c}
H \backslash G=\bigcup\{F \backslash G \mid F \in \gamma(H) \backslash \gamma(G)\} \\
I \backslash G=\bigcup\{F \backslash G \mid F \in \gamma(I) \backslash \gamma(G)\} .
\end{array}\right.
$$


Observe que, como $I \subseteq H$, necessariamente temos que $\gamma(I) \backslash \gamma(G) \subseteq \gamma(H) \backslash \gamma(G)$. Ainda, como $G \subsetneq I$, temos também que $\gamma(I) \backslash \gamma(G) \neq \emptyset$. Seja, então, $F^{*} \in \gamma(I) \backslash \gamma(G)$. Pela hipótese, vale que, para todo $F_{1}, F_{2} \in \gamma(H) \backslash \gamma(G)$, temos $F_{1} \backslash G=F_{2} \backslash G$. Em particular, temos, para todo $F_{1} \in \gamma(H) \backslash \gamma(G)$, a igualdade $F_{1} \backslash G=F^{*} \backslash G$. Portanto:

$$
\left\{\begin{array}{l}
\bigcup\{F \backslash G \mid F \in \gamma(H) \backslash \gamma(G)\}=F^{*} \backslash G \\
\bigcup\{F \backslash G \mid F \in \gamma(I) \backslash \gamma(G)\}=F^{*} \backslash G .
\end{array}\right.
$$

Consequentemente, temos que $I \backslash G=H \backslash G$. Como $I, H \supseteq G$, segue que $I=H$, o que mostra que $G \prec H$.

i) $\Rightarrow$ iii) : Suponha que $G$ seja coberto por $H$. Pela Proposição 6.7, podemos expressar $H$ como $H=G \cup \bigcup\{F \in \mathcal{F} \mid F \in \gamma(H) \backslash \gamma(G)\}$. Seja $F \in \gamma(H) \backslash \gamma(G)$. Claramente, temos que $G \subseteq G \cup F \subseteq H$. Como $G$ é coberto por $H$, temos então que $G \cup F=G$ ou que $G \cup F=H$. Como $F \notin \gamma(G)$, a possibilidade $G \cup F=G$ está descartada, e portanto, $G \cup F=H$.

O algoritmo de construção do diagrama de Hasse de $\overline{\mathcal{F}}$ se baseia no seguinte corolário do Teorema 6.8.

Corolário 6.9. Sejam $G \in \overline{\mathcal{F}}$ e $\mathcal{M}$ o multiconjunto definido por:

$$
\mathcal{M}=\{G \cup F \mid F \in \mathcal{F} \backslash \gamma(G)\} .
$$

Então, para todo $H \supsetneq G$, temos que $G \prec H$ se e somente se a multiplicidade de $H$ em $\mathcal{M}$ é igual a $|\gamma(H)|-|\gamma(G)|$.

Prova. Seja $H \supsetneq G$ e, portanto, $\gamma(G) \subseteq \gamma(H)$. Assim, temos a seguinte união disjunta:

$$
\mathcal{F} \backslash \gamma(G)=(\mathcal{F} \backslash \gamma(H)) \cup(\gamma(H) \backslash \gamma(G)) .
$$

E, consequentemente:

$$
\mathcal{M}=\{G \cup F \mid F \in \mathcal{F} \backslash \gamma(H)\} \cup\{G \cup F \mid F \in \gamma(H) \backslash \gamma(G)\} .
$$

No multiconjunto $\{G \cup F \mid F \in \mathcal{F} \backslash \gamma(H)\}$, o conjunto $H$ não ocorre nenhuma vez, já que um conjunto $G \cup F$ com $F \notin \gamma(H)$ não pode estar contido em $H$. Por outro lado, a equivalência entre i) e iii) no Teorema 6.8 garante que $G \prec H$ se e somente se o multiconjunto $\{G \cup F \mid F \in \gamma(H) \backslash \gamma(G)\}$ contém apenas $H$, e portanto, com multiplicidade igual a $|\gamma(H)|-|\gamma(G)|$.

No Algoritmo 9 se encontra descrita a segunda etapa. Sua correção segue a partir do Corolário 6.9. A apresentação ali contida é essencialmente matemática, e os detalhes de implementação, bem como a complexidade do Algoritmo 9 são abordados no Lema 6.10. 


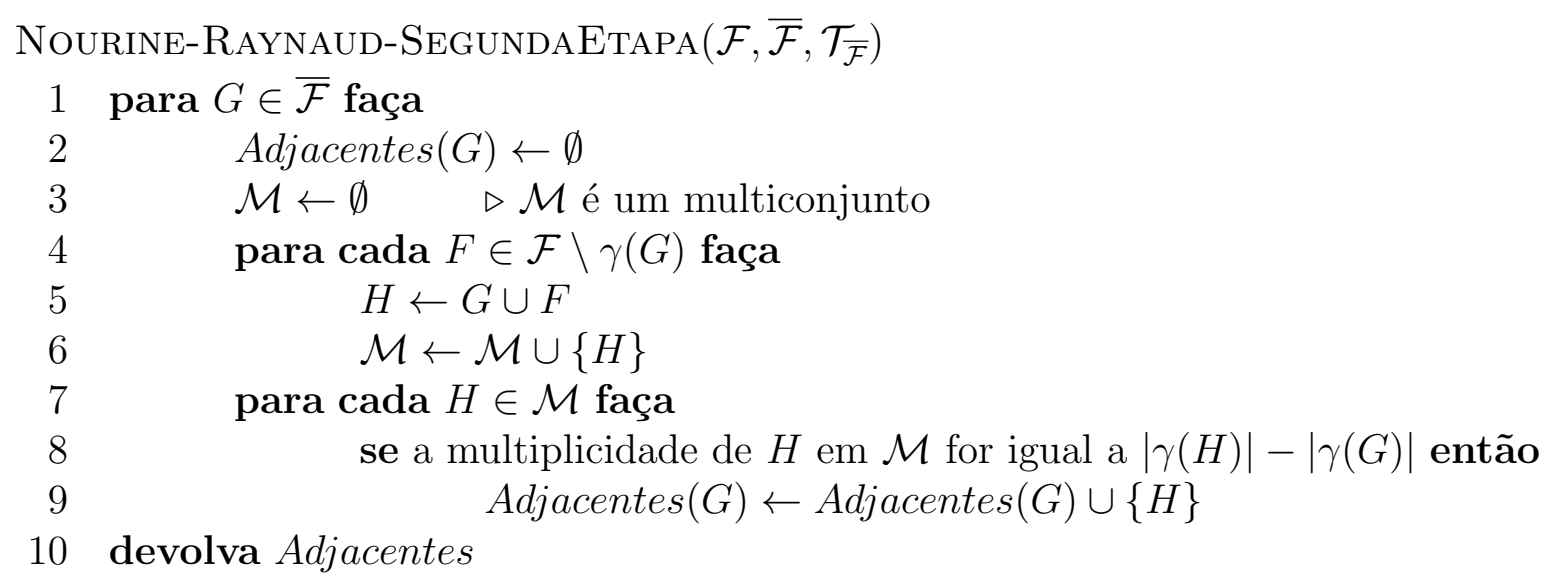

Algoritmo 9: Segunda etapa do algoritmo de Nourine e Raynaud.

Lema 6.10. O Algoritmo 9 pode ser implementado para consumir $O(|\overline{\mathcal{F}}| \cdot|\mathcal{F}| \cdot(|X|+|\mathcal{F}|))$ unidades de tempo.

Prova. Considere uma implementação cuja representação de $\mathcal{M}$ é realizada em uma lista ligada, assim como a representação de $\operatorname{Adjacentes}(G)$, para cada $G \in \overline{\mathcal{F}}$. Cada nó da lista ligada $\mathcal{M}$ representa uma ocorrência de um conjunto $H$ no multiconjunto $\mathcal{M}$.

Para cada $F \in \mathcal{F}$, a realização do teste $F \stackrel{?}{\in} \mathcal{F} \backslash \gamma(G)$ presente na linha 4 é realizável em tempo $O(|X|)$, já que temos $F \in \mathcal{F}$, e portanto, vale que $F \notin \gamma(G)$ se e somente se $F \nsubseteq G$. A operação de união na linha 5 pode ser executada em tempo $O(|X|)$, assim como a a linha 6 (na realidade, tempo $O(1)$ se representarmos apenas referências para os conjuntos $H \in \mathcal{M}$ ). Logo, as linhas 5 e 6 consomem, durante toda a execução, tempo $O(|\overline{\mathcal{F}}||\mathcal{F} \| X|)$.

A execução da linha 8 exige o conhecimento da multiplicidade de $H$ em $\mathcal{M}$, que pode ser obtido em $O(|X|)$ unidades de tempo se mantivermos, para cada vértice (final) $v_{H}$ de $\mathcal{T}_{\overline{\mathcal{F}}}$, um contador indicando a multiplicidade de $H$ em $\mathcal{M}$. Observe que isso pode ser feito se incrementarmos tal contador associado a $v_{H} \operatorname{logo}$ após a execução da linha 5 . Também observe que isto pode ser realizado em tempo $O(|X|)$, tendo em vista a complexidade da operação Pertence, estabelecida no Lema 6.5. Além disso, para evitarmos que os valores dos contadores propaguem para a iteração seguinte do laço da linha 1, bem como evitarmos eventuais adições de valores repetidos na lista $\operatorname{Adjacentes}(G)$, reinicializamos o contador da multiplicidade de $H$ para zero, logo após a execução da linha 8. Convém salientar que, durante a execução da linha 8, o valor de $|\gamma(H)|$ nunca é igual a $|\gamma(G)|$, pois $H=G \cup F$ com $F \in \mathcal{F}$ e $F \notin \gamma(G)$, o que implica que $\gamma(H) \supsetneq \gamma(G)$.

Ainda com relação à linha 8, para a obtenção dos valores de $|\gamma(G)|$ e $|\gamma(H)|$, conforme o Lema 6.5, podemos consumir tempo $O(|X|+|\mathcal{F}|)$. Logo, a linha 8 pode ser executada em $O(|X|+|\mathcal{F}|)$ unidades de tempo.

Para a linha 9, se Adjacentes $(G)$ for uma lista ligada, e representarmos $H$ explicitamente em tal lista, podemos obter complexidade $O(|X|)$ na execução de tal linha: basta mantermos uma referência para o último nó de tal lista. Se representarmos, em Adjacentes $(G)$, apenas uma referência para $H$, a complexidade de tempo $O(1)$ pode ser obtida.

Portanto a complexidade de tempo, nessa implementação, é dominada pela linha 8 que, por ser executada $O(|\overline{\mathcal{F}}| \cdot|\mathcal{F}|)$ vezes, confere complexidade de tempo $O(|\overline{\mathcal{F}}| \cdot|\mathcal{F}| \cdot(|X|+|\mathcal{F}|))$ 
a essa implementação do Algoritmo 9.

\subsubsection{Redução a partir do problema de construção de reticulado de conceitos}

O algoritmo para a construção de um reticulado de conceitos utilizando uma solução para o RFUF se baseia no próximo teorema. Lembramos que, conforme descrevemos na Seção 2.6, toda ordem parcial $\langle P, \leq\rangle$ possui uma ordem parcial dual, denotada por $\langle P, \geq\rangle$ ou $P^{\partial}$. A relação de ordem nesta ordem dual é definida a partir da ordem original: $x \geq y \Leftrightarrow y \leq x$.

Teorema 6.11. Seja $\mathbb{K}=(G, M, I)$ um contexto, e seja $\mathcal{F}=\left\{M \backslash g^{\prime} \mid g \in G\right\}$. Então,

$$
\langle\overline{\mathcal{F}}, \subseteq\rangle \cong\langle\operatorname{Int}(\mathbb{K}), \subseteq\rangle^{\partial} \cong \underline{\mathfrak{B}}(G, M, I) .
$$

De maneira dual, se $\mathcal{F}=\left\{G \backslash m^{\prime} \mid m \in M\right\}$, então

$$
\langle\overline{\mathcal{F}}, \subseteq\rangle \cong\langle\operatorname{Ext}(\mathbb{K}), \subseteq\rangle^{\partial} \cong \underline{\mathfrak{B}}(G, M, I)^{\partial} .
$$

Prova. Vamos mostrar a primeira afirmação, a segunda segue de maneira análoga. Seja $\operatorname{Int}(\mathbb{K})$ o conjunto das intenções de $\mathbb{K}$, e seja $\overline{\operatorname{Int}(\mathbb{K})}$ o conjunto de complementos de intenções, isto é

$$
\overline{\operatorname{Int}(\mathbb{K})}=\{M \backslash B \mid B \in \operatorname{Int}(\mathbb{K})\} .
$$

É fácil mostrar que $\overline{\operatorname{Int}(\mathbb{K})}$ é um reticulado anti-isomorfo a $\operatorname{Int}(\mathbb{K})$. De fato, a função $\varphi: \operatorname{Int}(\mathbb{K}) \rightarrow \overline{\operatorname{Int}(\mathbb{K})}^{\partial}$ dada por $\varphi(B)=M \backslash B$ pode ser facilmente verificada como um isomorfismo de ordem.

Da Proposição 5.1, temos:

$$
\operatorname{Int}(\mathbb{K})=\left\{\bigcap_{g \in J} g^{\prime} \mid J \subseteq G\right\},
$$

que implica que:

$$
\begin{aligned}
\overline{\operatorname{Int}(\mathbb{K})} & =\left\{M \backslash \bigcap_{g \in J} g^{\prime} \mid J \subseteq G\right\} \\
& =\left\{\bigcup_{g \in J} M \backslash g^{\prime} \mid J \subseteq G\right\} \text { (lei de de Morgan) } \\
& =\left\{\bigcup_{F \in J} F \mid J \subseteq\left\{M \backslash g^{\prime} \mid g \in G\right\}\right\}=\overline{\mathcal{F}} .
\end{aligned}
$$

Portanto, $\langle\overline{\mathcal{F}}, \subseteq\rangle$ é anti-isomorfo a $\langle\operatorname{Int}(\mathbb{K}), \subseteq\rangle$ e, assim, isomorfo a $\underline{\mathfrak{B}}(G, M, I)$.

O algoritmo que reduz o problema de se construir o reticulado de conceitos ao RFUF, com a complexidade prometida está esclarecido no próximo corolário.

Corolário 6.12. Usando-se apropriadamente os Algoritmos 8 e 9, obtemos um algoritmo de tempo $O(|\mathfrak{B}| \cdot|G| \cdot|M|)$ para o problema da construção do reticulado de conceitos $\underline{\mathfrak{B}}(G, M, I)$. 
Prova. Considere o seguinte algoritmo:

1. Se $|G| \leq|M|$, utilizam-se os Algoritmos 8 e 9 para o RFUF com $\mathcal{F}=\left\{M \backslash g^{\prime} \mid g \in G\right\}$.

2. Se $|G|>|M|$, utilizam-se o Algoritmos 8 e 9 para o RFUF com $\mathcal{F}=\left\{G \backslash m^{\prime} \mid m \in M\right\}$. Realiza-se um pós-processamento no diagrama de Hasse obtido, a fim de inverter a direção de cada arco, devido à obtenção do dual do reticulado de conceitos.

No caso 1 , temos $|\mathcal{F}|=|G|,|X|=|M|$ e portanto $|X|+|\mathcal{F}|=O(|M|)$, já que $|M| \geq|G|$, e a complexidade desta solução é de $|\overline{\mathcal{F}}| \cdot|\mathcal{F}| \cdot(|X|+|\mathcal{F}|)=O(|\mathfrak{B}| \cdot|G| \cdot|M|)$ unidades de tempo, devido aos Lemas 6.6 e 6.10 .

No caso 2, temos $|\mathcal{F}|=|M|,|X|=|G|$ e portanto $|X|+|\mathcal{F}|=O(|G|)$, já que $|G|>|M|$, e a complexidade desta solução é de $|\overline{\mathcal{F}}| \cdot|\mathcal{F}| \cdot(|X|+|\mathcal{F}|)=O(|\mathfrak{B}| \cdot|M| \cdot|G|)$ unidades de tempo, devido aos Lemas 6.6 e 6.10 . 



\section{Capítulo 7}

\section{Contextos com número polinomial de conceitos}

A correspondência entre bicliques maximais e conceitos, exposta na Proposição 2.2, permite o transporte de resultados da teoria dos grafos para o cenário de Formal Concept Analysis. Vimos que existe uma família de grafos bipartidos $\left\{G_{i}\right\}_{i \in I}$ para a qual existe uma função $f(n)=\Omega\left(c^{n}\right)$, onde $c>1$ é uma constante e $n=n(i)$ é o número de vértices de cada membro da família, tal que todo grafo $G_{i}$ possui um número de bicliques maximais maior ou igual a $f(n)$. De fato, durante a exposição do Corolário 2.10, vimos que este é o caso para a família dos grafos coroa. Aplicações interativas de FCA a grafos que pertençam a uma tal família são inviáveis mesmo para grafos com poucos vértices, face ao crescimento exponencial dos tamanhos de reticulados de conceitos associados. Neste capítulo, nosso objetivo é encontrar famílias de casos mais tratáveis, isto é, desejamos encontrar famílias de grafos bipartidos que possuam um número polinomial de bicliques maximais. Mais precisamente, queremos encontrar famílias $\left\{G_{i}\right\}_{i \in I}$ de grafos bipartidos e uma função polinomial $p(n)$ de maneira que cada $G_{i}$ possua um número de bicliques maximais menor ou igual a $p(n)$.

Lembramos que cada membro $C G(2 k)$ da família dos grafos coroa é $(k-1)$-regular, e que portanto possui grau máximo elevado, proporcional ao número de vértices do grafo. É então natural investigar se ao limitarmos o grau máximo de um grafo, podemos obter uma cota superior polinomial em $n$ para seu número de bicliques maximais. De fato, mostraremos a existência de uma tal cota neste capítulo. Ademais, mostraremos dois outros resultados da mesma natureza, dados em função do número de arestas e de uma medida chamada arboricidade. Tais resultados são devidos respectivamente, a Schütt e a David Eppstein. Para a cota de Eppstein, será mostrado um limite inferior que prova que esta cota é quase justa, em um sentido preciso. Depois, provaremos uma cota superior em função do que definiremos como a convexidade do grafo bipartido em questão. Serão mostradas famílias de grafos para a qual este novo resultado oferece uma melhor cota que as cotas do grau máximo, de Eppstein e de Schütt. Em particular, serão mostradas famílias para as quais a cota baseada em convexidade é cúbica, enquanto que as demais são exponenciais. 


\subsection{Definições e a cota da classe mínima}

Seja $G=(V, E)$ um grafo não orientado. Como anteriormente, uma aresta $\left\{v_{1}, v_{2}\right\} \in E$ é denotada simplesmente por $v_{1} v_{2}$ e, neste caso, os vértices $v_{1}$ e $v_{2}$ são chamados de pontas. Dizemos ainda que $v_{1}$ e $v_{2}$ são vizinhos ou adjacentes, e que a aresta $v_{1} v_{2}$ incide em $v_{1} \mathrm{e}$ em $v_{2}$. Se $V$ admitir uma partição $\{U, W\}$ de maneira que toda aresta de $G$ possua uma ponta em $U$ e outra em $W$, então dizemos que o grafo $G$ é $(U, W)$-bipartido. Neste trabalho, um grafo bipartido é um grafo $G$ cujo conjunto de vértices se encontra sob uma tal partição fixada, e denotamos isto por $G=(U, W, E)$. Os conjuntos $U$ e $W$ são chamados de classes de vértices, ou simplesmente classes. Utilizaremos os símbolos $V(G)$ e $E(G)$ para nos referirmos, respectivamente, ao conjunto de vértices e ao conjunto de arestas de $G$.

Considere um grafo bipartido $(U, W, E)$. Lembramos que uma biclique de um tal grafo é um $\operatorname{par}(A, B)$ com $A \subseteq U, B \subseteq W$ e $u w \in E$ para todo $u \in A, w \in B$. O conjunto $A$ é chamado de primeira coordenada, e $B$ chamado de segunda coordenada. Para todo vértice $v$ do grafo em questão, dizemos que uma biclique $(A, B)$ contém $v$ se $v \in A \cup B$. Note que a definição de biclique não impede a ocorrência de coordenadas iguais ao conjunto vazio. Essa ocorrência potencial de coordenadas vazias pode parecer artificial a princípio, mas se prova útil para a realização de um paralelo entre FCA e teoria dos grafos, como o que foi realizado na Proposição 2.2. Bicliques com coordenadas vazias requerem, muitas vezes, consideração especial durante a demonstração de um resultado. Desta maneira, se $A \neq \emptyset$ e $B \neq \emptyset$, dizemos que $(A, B)$ é uma biclique com arestas. Caso contrário, dizemos que $(A, B)$ é uma biclique sem arestas.

Recordamos que uma biclique maximal é uma biclique $(A, B)$ tal que, para toda biclique $(C, D)$ a seguinte implicação vale: $C \supseteq A$ e $D \supseteq B \Rightarrow C=A$ e $D=B$. Neste capítulo, utilizaremos com frequência o símbolo $\mathfrak{B}$ para denotar o conjunto de bicliques maximais de um grafo bipartido, quando estiver claro, a partir do contexto, qual é o grafo em questão.

No caso de grafos bipartidos, convém utilizar a seguinte notação para designarmos a vizinhança de vértices $u \in U$ e $w \in W$ :

$$
N(u)=\{w \in W \mid u w \in E\} \quad \text { e } \quad N(w)=\{u \in U \mid u w \in E\} .
$$

Por convenção, no caso de $A$ e $B$ serem o conjunto vazio, as intersecções $\cap_{u \in A} N(u)$ e $\cap_{w \in B} N(w)$ valem respectivamente, $W$ e $U$.

Pela caracterização contida na Proposição 2.2, temos que uma biclique $(A, B)$ é maximal se e só se $A=\cap_{w \in B} N(w)$ e $B=\cap_{u \in A} N(u)$. Esta caracterização mostra que, assim como em conceitos, o conhecimento de uma das coordenadas de uma biclique maximal automaticamente determina a outra coordenada. Desta maneira, temos uma primeira cota, bastante elementar, para o número de bicliques maximais:

Proposição 7.1 (Cota da classe mínima). Seja $(U, W, E)$ um grafo bipartido. Então, temos que $|\mathfrak{B}| \leq 2^{\min \{|U|,|W|\}}$.

Dados dois grafos $G=(V, E)$ e $G^{\prime}=\left(V^{\prime}, E^{\prime}\right)$, se existir uma bijeção $\phi: V \rightarrow V^{\prime}$ tal que, para todo $v_{1}, v_{2} \in V$, vale a equivalência $v_{1} v_{2} \in E \Leftrightarrow \phi\left(v_{1}\right) \phi\left(v_{2}\right) \in E^{\prime}$, então dizemos que $G$ e $G^{\prime}$ são grafos isomorfos. Durante este capítulo, falaremos sobre famílias notáveis de grafos. Chamamos de grafo bipartido completo com $k$ e l vértices um grafo bipartido $(U, W, E)$ com 
$|U|=k,|W|=l$ e $u w \in E$ para todo $u \in U, w \in W$. Não fazemos distinção entre grafos isomorfos e, assim, para fixados $k, l \in \mathbb{N}$, um tal grafo será chamado de $o$ grafo bipartido completo com $k$ e $l$ vértices, e o denotaremos por $K_{k, l}$. A subfamília $\left\{K_{1, l}\right\}_{l \in \mathbb{N}}$ será chamada de familia das estrelas.

O grau de um vértice $v$, denotado $g(v)$, é o número de arestas que incidem em $v$. Denotamos por $\Delta$ o grau máximo de um grafo, isto é, o máximo dentre os graus de seus vértices. Na próxima seção veremos, em particular, uma cota superior para $|\mathfrak{B}|$ em função de $\Delta$.

\subsection{As cotas dos graus máximos}

Nesta seção, trataremos de cotas superiores para $|\mathfrak{B}|$ em função do grau máximo de um grafo bipartido. Antes de expormos o primeiro resultado, precisamos de um resultado que caracteriza as bicliques maximais sem arestas.

Proposição 7.2. Seja $G=(U, W, E)$ um grafo bipartido. $O$ par $(A, \emptyset)$ é sempre uma biclique, e é maximal se e somente se $A=U$ e $\cap_{u \in U} N(u)=\emptyset$. Analogamente, $(\emptyset, B)$ é sempre uma biclique, e é maximal se e só se $B=W$ e $\cap_{w \in W} N(w)=\emptyset$.

Prova. Segue diretamente a partir da Proposição 2.2.

Uma consequência imediata da Proposição 7.2 é que todo grafo bipartido possui no máximo duas bicliques maximais sem arestas. Assim, uma primeira tentativa para limitar o número de bicliques maximais de um grafo bipartido poderia, então, ser através da limitação do próprio número de bicliques (não necessariamente maximais) com arestas. A próxima proposição exibe uma cota para este número.

Proposição 7.3. Seja $G=(U, W, E)$ um grafo bipartido com $n$ vértices, e seja $\Delta o$ grau máximo de $G$. Então, o número de bicliques com arestas de $G$ é menor ou igual a $\min \{|U|,|W|\} \cdot 2^{\Delta-1}\left(2^{\Delta}-1\right)=O\left(n \cdot 2^{2 \Delta}\right)$.

Prova. Sem perda de generalidade, suponha que $|U| \leq|W|$. Seja $u \in U$. O número de segundas coordenadas de bicliques com arestas que contém $u$ é menor ou igual a $2^{\Delta}-1$. De fato, se $B$ for uma tal segunda coordenada, então vale que $B \subseteq N(u)$ e que $B \neq \emptyset$. Suponha agora que fixamos uma tal segunda coordenada, $B$. Temos, assim, algum vértice $w \in B$ e, portanto, o número de primeiras coordenadas de bicliques $(A, B)$ é $2^{\Delta-1}$, uma vez que obrigatoriamente $u \in A$ e $A \subseteq N(w)$. Temos, assim, não mais do que $2^{\Delta-1}\left(2^{\Delta}-1\right)$ escolhas para bicliques com arestas que contém $u$. Repetindo este argumento para cada $u \in U$, obtemos o resultado.

Uma consequência das Proposições 7.2 e 7.3 é que o número de bicliques maximais de um grafo bipartido nunca excede $n 2^{2 \Delta}+2$. É de se esperar que esta cota possa ser melhorada, já que estamos limitando o número de quaisquer bicliques com arestas. De fato, veremos no Lema 7.4 uma melhor cota para $|\mathfrak{B}|$ em termos de $\Delta$, que não chega a limitar o número de bicliques (não necessariamente maximais) com arestas.

Uma das motivações para a procura de um limite superior específico para $|\mathfrak{B}|$, isto é, sem forçarmos um limite para o número de bicliques com arestas, é a facilidade de se exibir 
famílias de grafos que possuem simultaneamente muitas bicliques com arestas mas poucas bicliques maximais. Um caso muito simples é a família das estrelas $K_{1, k}$. Entretanto, o uso da cota da classe mínima, vista na Proposição 7.1, nos dá um resultado bastante justo para o número de bicliques maximais dos grafos da família $K_{1, k}$. Todavia, se aplicarmos a mesma cota, para um membro da família descrita na Figura 7.1, teremos uma cota para $|\mathfrak{B}|$ que é exponencial no número de vértices do grafo em questão, que contudo, pode-se verificar, possui exatamente $n / 2+1$ bicliques maximais ${ }^{1}$.

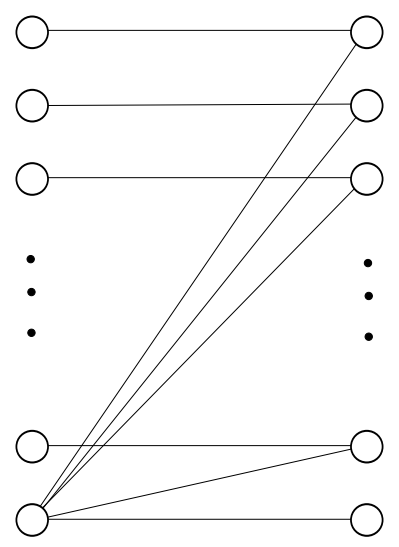

Figura 7.1: Família de grafos com grau máximo elevado, muitas bicliques com arestas, mas poucas bicliques maximais.

Apesar dos grafos de tal família possuirem grau máximo elevado, isto é causado apenas por um vértice de $U$, enquanto que os graus de vértices pertencentes a $W$ são pequenos. No Lema 7.4 a seguir, vemos que é possível considerar separadamente o grau máximo de $U$ e de $W$, e obter cotas superiores específicas para o número de bicliques maximais em função destes. Ademais, sua cota $n 2^{\Delta}$ é melhor que a cota $n 2^{2 \Delta}$ obtida para $|\mathfrak{B}|$ como consequência da Proposição 7.3.

Lema 7.4 (Cotas dos graus máximos). Seja $G=(U, W, E)$ um grafo bipartido, e sejam $\Delta_{U}$ o grau máximo dos vértices em $U$ e $\Delta_{W}$ o grau máximo dos vértices em $W$. Então,

$$
|\mathfrak{B}| \leq|U| \cdot\left(2^{\Delta_{U}}-1\right)+2=O\left(|U| \cdot 2^{\Delta_{U}}\right) .
$$

Analogamente,

$$
|\mathfrak{B}| \leq|W| \cdot\left(2^{\Delta_{W}}-1\right)+2=O\left(|W| \cdot 2^{\Delta_{W}}\right) .
$$

Em particular, temos que $|\mathfrak{B}|=O\left(n \cdot 2^{\Delta}\right)$, onde $n=|U|+|W|$ é o número de vértices de $G$.

Prova. Mostraremos a primeira desigualdade. Inicialmente, estimamos o número de bicliques maximais com arestas. O número de bicliques maximais com arestas que contém um vértice fixado $u \in U$, é no máximo $2^{\Delta_{U}}-1$. De fato, para toda biclique $(A, B)$ nestas condições, temos que $B \subseteq N(u)$ e que $B \neq \emptyset$. Portanto, há no máximo $2^{\Delta_{U}}-1$ possibilidades para a escolha de $B$. Como a escolha de $B$ automaticamente determina a escolha de $A$ no caso de

\footnotetext{
${ }^{1}$ Cada um dos $\frac{n}{2}-1$ vértices de $U$ com grau um participam de um $K_{2,1}$ maximal. Fora estes, há somente um $K_{1,|W|}$ maximal e um $K_{|U|, 0}$ maximal.
} 
bicliques maximais, o argumento segue. Aplicando esse raciocínio para cada $u \in U$, temos que o número de bicliques maximais com arestas de $G$ é no máximo $|U| \cdot\left(2^{\Delta_{U}}-1\right)$. Utilizando a Proposição 7.2, temos no máximo mais duas bicliques maximais: as sem arestas.

Se um grafo bipartido possuir uma de suas classes com vértices de grau pequeno, então a cota associada a esta classe fornecida pelo Lema 7.4 será candidata para prover uma boa cota superior para $|\mathfrak{B}|$. No entanto, é possível que as duas classes possuam algum vértice com grau elevado, como na família representada na Figura 7.2, mas possuam poucas bicliques maximais ${ }^{2}$.

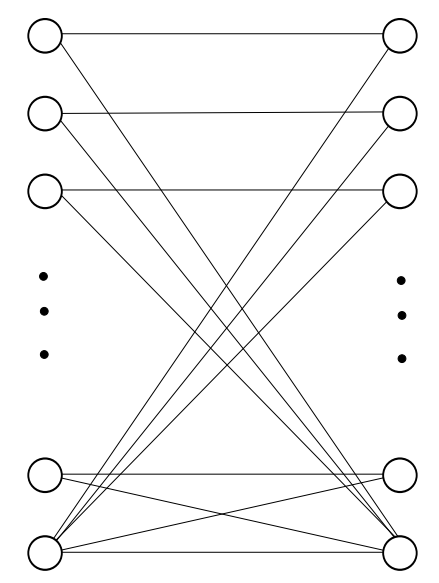

Figura 7.2: Família de grafos com $\Delta_{U}$ e $\Delta_{W}$ em $\Omega(n)$.

Famílias de grafos como a da Figura 7.2 motivam a busca para novas cotas superiores para $|\mathfrak{B}|$. Apesar de tal família não possuir grau máximo limitado por uma constante, veremos que tal família é "esparsa". Na próxima seção, abordaremos a relação entre "esparsidade" e o número de bicliques maximais.

\subsection{Família esparsa com $|\mathfrak{B}|$ superpolinomial}

Seja $\left\{G_{i}\right\}_{i \in I}$ uma família de grafos, com cada um de seus membros possuindo $n=n(i)$ vértices. Neste capítulo, dizemos que tal família é esparsa se existir uma função em $O(n)$ que limita superiormente a cardinalidade do conjunto de arestas de cada $G_{i}$. Observe que um grafo arbitrário da família retratada na Figura 7.2 possui $\frac{3 n}{2}-2$ arestas.

Com frequência, famílias esparsas são consideradas aquelas cujos membros possuem $o\left(n^{2}\right)$ arestas, enquanto que membros de famílias densas possuem $\Omega\left(n^{2}\right)$ arestas. Nesta seção, veremos que mesmo exigindo $O(n)$ arestas para uma família se qualificar como sendo esparsa, ainda assim podemos exibir um exemplo de família esparsa com número de bicliques maximais superpolinomial em $n$.

Considere um grafo bipartido $(U, W, E)$. O seguinte fato proveniente da teoria dos grafos é de imediata demonstração:

$$
\sum_{u \in U} g(u)=\sum_{w \in W} g(w)=|E| .
$$

\footnotetext{
${ }^{2}$ Uma contagem semelhante à realizada sobre a Figura 7.1 mostra que $|\mathfrak{B}|=\frac{n}{2}+1$.
} 
A equação (7.2) implica que famílias de grafos bipartidos com grau máximo de $U$ (de $W$ ) limitado por uma mesma constante são esparsas. Observe que uma das consequências das cotas dos graus máximos (Lema 7.4) é que, se os grafos de uma família possuirem grau máximo de $U$ (de $W$ ) limitados por uma constante, então o número de bicliques maximais de cada grafo desta família é $O(n)$. Assim, pode-se supor que, a esparsidade da família possa ser usada como generalização da condição de grau máximo constante, e ainda assim obter-se o mesmo resultado de $|\mathfrak{B}|=O(n)$, para um membro qualquer de tal família. No entanto, veremos um contra-exemplo para esta pretensa generalização.

Considere um grafo $G$, de $n$ vértices, e suponha que uma das componentes ${ }^{3}$ de $G$ é o grafo coroa $C G(2\lfloor\sqrt{n} / 2\rfloor)$, cujo número de bicliques maximais é $2^{\left\lfloor\frac{\sqrt{n}}{2}\right\rfloor} \geq 2^{\frac{\sqrt{n}}{2}-1}=\sqrt{2}^{\sqrt{n}-2}$. Note que uma biclique maximal com arestas desta componente também é uma biclique maximal com arestas de $G$. Além disso, note que $|E(G)|=(\lfloor\sqrt{n} / 2\rfloor)(\lfloor\sqrt{n} / 2\rfloor-1)=O(n)$. Se as outras componentes de $G$ possuirem, ao todo, uma quantidade $O(n)$ de arestas, teremos que a família descrita por esta construção é esparsa, e não há polinômio que limite superiormente o valor de $|\mathfrak{B}|$ de cada membro desta família. Um exemplo desta construção está retratado na Figura 7.3.

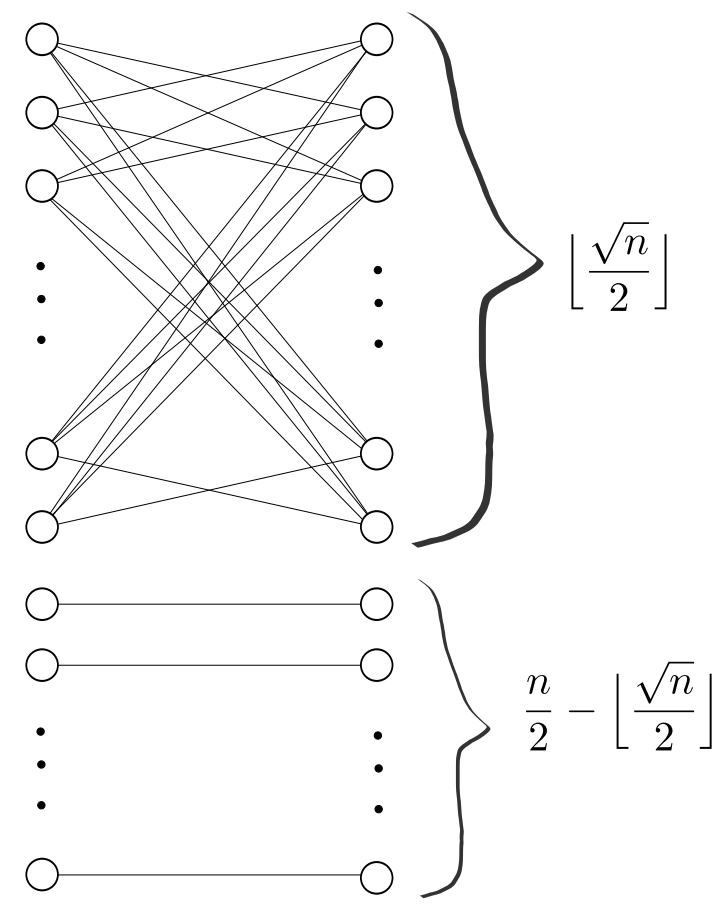

Figura 7.3: Família esparsa de grafos com $\Omega\left(\sqrt{2}^{\sqrt{n}}\right)$ bicliques maximais.

Na próxima seção, exporemos um resultado, devido a David Eppstein, que é capaz de prover boas cotas para o número de bicliques maximais de famílias como a descrita na Figura 7.2.

\footnotetext{
${ }^{3}$ A definição de componente (conexa) utilizada aqui, e em todo o texto, é a usual, não devendo suscitar dúvidas. Postergamos esta definição para a Seção 7.5, pouco antes da apresentação do Lema 7.7, onde este conceito é mais utilizado.
} 


\subsection{A arboricidade de um grafo}

Abordaremos nesta seção grafos não necessariamente bipartidos, e precisaremos de algumas definições adicionais. Seja $G=(V, E)$ um grafo. Dizemos que $H=(W, F)$ é um subgrafo de $G$, e denotamos isto por $H \subseteq G$, se $W \subseteq V$ e $F \subseteq E \cap\left\{\left\{w_{1}, w_{2}\right\} \mid w_{1}, w_{2} \in W\right\}$. Se $H \neq G$, dizemos adicionalmente que $H$ é um subgrafo próprio, e escrevemos $H \subsetneq G$.

Dado um conjunto de vértices $S \subseteq V(G)$, o subgrafo de $G$ que possui $S$ como vértices, e como arestas possui todas aquelas que possuem ambas as pontas em $S$, é chamado de subgrafo (de $G$ ) induzido por $S$ e denotado por $G[S]$. Um subgrafo $H$ de $G$ é dito induzido se existir $S \subseteq V(G)$ tal que $H=G[S]$.

Um caminho é uma sequência de vértices distintos $\left(v_{1}, v_{2}, \ldots, v_{k}\right)$ tal que $v_{i} v_{i+1} \in E$ para todo $i \in\{1, \ldots, k-1\}$, e os vértices $v_{1}$ e $v_{k}$ são seus extremos. Um circuito é uma sequência de vértices $\left(v_{1}, v_{2}, \ldots, v_{k}\right)$ tal que a subsequência de seus primeiros $k-1$ elementos é um caminho, $v_{k}=v_{1}$ e $v_{k-1} v_{k} \in E$. Uma floresta é um subgrafo que não possui circuitos.

Seja $k \in \mathbb{N}^{*}$. Definimos que um grafo $G$ é $k$-floresta-decomponivel se $E(G)$ puder ser escrito como

$$
E(G)=\bigcup_{i=1}^{k} E\left(F_{i}\right)
$$

onde cada $F_{i}$ é uma floresta, para $i=1, \ldots, k$ e $E\left(F_{i}\right) \cap E\left(F_{j}\right)=\emptyset$ para todo $i \neq j$. Neste caso, chamamos $F_{1}, \ldots, F_{k}$ de $k$-floresta-decomposição de $G$. Convencionamos que um grafo $G$ é 0-floresta-decomponível se $E(G)=\emptyset$. Por definição, a arboricidade de um grafo $G$, denotada $\Upsilon(G)$, é

$$
\Upsilon(G)=\min \{k \in \mathbb{N}: G \text { é } k \text {-floresta-decomponível }\} \text {. }
$$

Na Figura 7.4 se encontra um exemplo de um grafo sob uma 2-floresta-decomposição, onde arestas pontilhadas pertencem a uma das florestas, e as arestas com traço cheio pertencem à outra. Observe que este grafo é membro ${ }^{4}$ da família descrita na Figura 7.2, e também que tal grafo não admite uma 1-floresta-decomposição, uma vez que ele possui um circuito. Assim, a arboricidade deste grafo é dois, assim como a de qualquer membro da família descrita na Figura 7.2, por argumento idêntico.

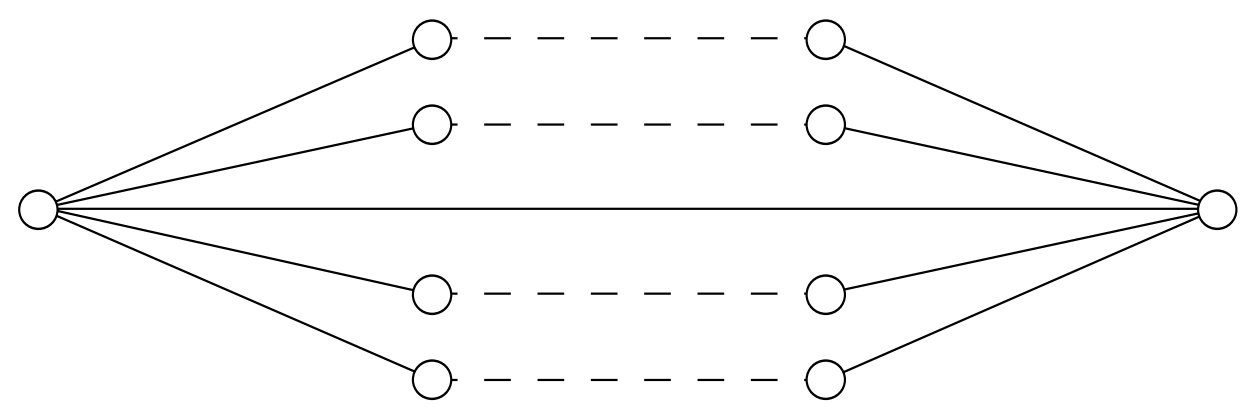

Figura 7.4: Uma 2-floresta-decomposição de um membro da família descrita na Figura 7.2.

\footnotetext{
${ }^{4}$ Nesta representação, os vértices de grau elevado foram movidos para lados opostos.
} 
O resultado presente no Teorema 7.5 foi demonstrado por Nash-Williams em [NW64], como sendo uma consequência de um resultado anterior, demonstrado independentemente pelo próprio autor em [NW61] e por Tutte [Tut61]. Uma demonstração recente desses resultados pode ser encontrada em [Die10].

Teorema 7.5 (Nash-Williams). Um grafo $G$ é $k$-floresta-decomponivel se e somente se $|E(H)| \leq k(|V(H)|-1)$, para todo subgrafo $H \subseteq G$.

Como consequência, temos o seguinte corolário, que provê uma fórmula para o cálculo da arboricidade de um grafo.

Corolário 7.6. Seja G um grafo. Então:

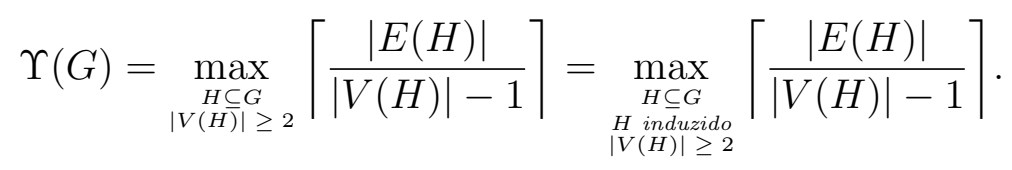

Prova. A primeira igualdade é clara. O máximo pode ser restrito apenas a subgrafos induzidos pois, para um subgrafo qualquer $H \subseteq G$, o valor da fração $|E(H)| /(|V(H)|-1)$ é menor ou igual à fração correspondente do subgrafo induzido por $V(H)$, isto é, $G[V(H)]$.

Utilizando o Corolário 7.6, é possível comparar a arboricidade do grafo da Figura 7.3, quando mostramos um contra-exemplo relacionado à esparsidade, e o grafo da Figura 7.2, que possui um vértice de grau elevado em cada classe. Seja $G$ um membro da família descrita na Figura 7.3, e $n$ o número de seus vértices. Seja, ainda, $k=\left\lfloor\frac{\sqrt{n}}{2}\right\rfloor$, e $H$ o subgrafo de $G$ isomorfo ao grafo coroa $C G(2 k)$. O máximo presente no Corolário 7.6 pode ser limitado inferiormente pelo valor atingido pelo subgrafo $H: \Upsilon(G) \geq\left\lceil\frac{k(k-1)}{2 k-1}\right\rceil=\Omega(k)$. Portanto, $\Upsilon(G)=\Omega(\sqrt{n})$. Assim, apesar de as famílias descritas nas Figuras 7.3 e 7.2 apresentarem a propriedade de serem esparsas, as arboricidades de seus membros são bastante diferentes, pois os grafos da família da Figura 7.2 têm arboricidade 2, enquanto que os da Figura 7.3 têm arboricidade $\Omega(\sqrt{n})$.

\subsection{A cota da arboricidade}

Foram considerados por Eppstein, em [Epp94], grafos não necessariamente bipartidos e seus chamados subgrafos bipartidos completos maximais, algo similar à nossa definição de biclique maximal. Faremos agora mais definições, a fim de detalhar a linguagem utilizada por Eppstein.

Seja $G=(V, E)$ um grafo. Dizemos que $S \subseteq V$ é independente em $G$ se $v_{1} v_{2} \notin E$ para todo $v_{1}, v_{2} \in S$. Um subgrafo bipartido completo de $G$ é um subgrafo $(W, F)$ de $G$ tal que $W$ admite uma bipartição $\left\{W_{1}, W_{2}\right\}$, com $W_{1}$ e $W_{2}$ independentes em $(W, F)$, e com cada vértice de $W_{1}$ sendo adjacente, em $(W, F)$, a cada vértice de $W_{2}$. Um subgrafo bipartido completo maximal é um subgrafo bipartido completo que não é subgrafo próprio de nenhum subgrafo bipartido completo. Um exemplo está representado na Figura 7.5. Nesta figura, temos destacado o subgrafo bipartido completo maximal $(W, F)$, com conjunto de vértices $W=\left\{v_{1}, v_{2}, v_{3}, v_{4}, v_{5}, v_{6}\right\}=\left\{v_{1}, v_{2}, v_{3}, v_{4}\right\} \cup\left\{v_{5}, v_{6}\right\}$ e conjunto de arestas, representado com pontilhado na Figura 7.5, sendo $F=\left\{v_{i} v_{j} \mid v_{i} \in\left\{v_{1}, v_{2}, v_{3}, v_{4}\right\}, v_{j} \in\left\{v_{5}, v_{6}\right\}\right\}$. 


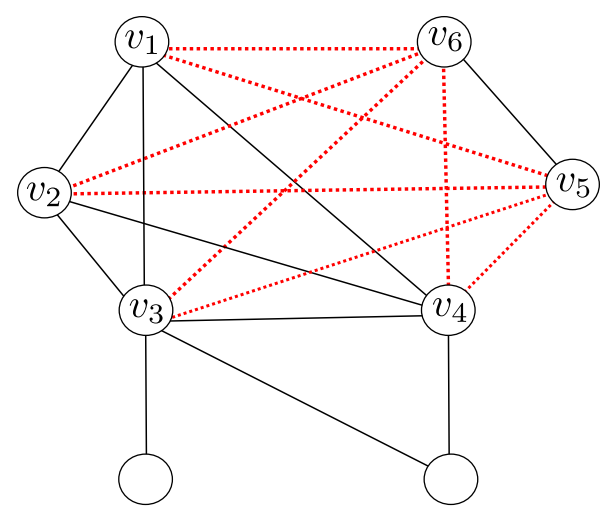

Figura 7.5: Um grafo com um subgrafo bipartido completo maximal destacado.

Nossa definição de biclique é aplicável apenas para grafos bipartidos, o que não ocorre com os subgrafos bipartidos completos abordados por Eppstein. Todavia, nossa definição de biclique apresenta a vantagem de ser próxima à definição de conceitos, em Formal Concept Analysis, de maneira que o conteúdo do paralelo realizado na Proposição 2.2 é de fácil exposição.

Em [Epp94], Eppstein mostra uma cota superior para o número de subgrafos bipartidos completos maximais de um grafo em função da sua arboricidade. A semelhança formal entre subgrafos bipartidos completos maximais e bicliques maximais torna possível uma sutil adaptação deste resultado, de forma a obter-se uma cota superior para o número de bicliques maximais de um grafo bipartido.

Neste trabalho, nosso interesse principal incide em grafos bipartidos e, portanto, não apresentaremos os resultados de [Epp94] utilizando a linguagem dos subgrafos bipartidos completos maximais. Ao invés, continuaremos o emprego da linguagem já desenvolvida sobre bicliques maximais. Isso implica a necessidade de adaptarmos e adicionarmos uma hipótese a dois dos resultados que apresentaremos. Mais especificamente, o Lema 7.9 e o Teorema 7.10 são resultados baseados nos resultados de [Epp94], mas com a hipótese adicional de bipartição do grafo, a fim de haver conformidade destes resultados com nossas definições. A princípio, uma adição de hipótese pode levantar indagações sobre a possibilidade de se provar uma conclusão mais forte. Em resposta a isso, mostraremos na Seção 7.6 que este aumento de hipóteses não permite melhores conclusões do que a tese do Teorema 7.10, que é o resultado sobre a cota superior para $|\mathfrak{B}|$ em função da arboricidade.

Os dois primeiros lemas que mostraremos são resultados que não dependem da propriedade de bipartição do grafo. Antes de mostrá-los, precisamos de definições adicionais, principalmente sobre grafos orientados.

Seja $G$ um grafo. Se existir, em $G$, um caminho com extremos $v_{1}$ e $v_{2}$, então dizemos que $v_{1}$ e $v_{2}$ se acessam. Se cada um dos vértices de um grafo acessa todos os outros, dizemos que o grafo em questão é conexo. Os subgrafos conexos maximais de um grafo são chamados de componentes conexas ou, simplesmente, componentes. Cada componente de uma floresta será chamada de árvore.

Se houver uma orientação de $G$, clara a partir do contexto, e quisermos considerar $G$ sob tal orientação, utilizaremos o símbolo $\vec{G}$ para denotá-lo sob tal orientação. Para um grafo orientado $(V, A)$, cada arco $\left(v_{1}, v_{2}\right) \in A$ será denotado por $v_{1} v_{2}$. O conjunto de vizinhos de 
um vértice $u$ é denotado e definido da seguinte forma: $\vec{N}(u)=\{v \in V \mid u v \in A\}$. Além disso, o grau de saída de um vértice $u \in V$, denotado $g^{+}(u)$, é o número de arcos em $A$ da forma $u v$, com $v \in V$, isto é, $|\vec{N}(u)|$. O grau de entrada de $u$, denotado $g^{-}(u)$, é definido analogamente. Uma orientação d-limitada de $G$ é uma orientação de $G$ na qual cada vértice possui grau de saída no máximo $d$.

Lema 7.7. Se um grafo $G$ possui arboricidade $\Upsilon$, então $G$ possui uma orientação $\Upsilon$-limitada.

Prova. Considere uma $\Upsilon$-floresta-decomposição de $G, E(G)=\bigcup_{i=1}^{\Upsilon} E\left(F_{i}\right)$. Para cada componente (isto é, cada árvore) $T$ de cada floresta $F_{i}$, considere um vértice arbitrário $r \in V(T)$, que será definido como sendo a raiz associada a $T$. Definimos uma orientação para $T$ : os arcos de $\vec{T}$ estão orientados em direção a $r$. É claro que, por termos definido uma orientação para cada árvore de cada floresta da decomposição, acabamos por definir uma orientação para todo o grafo $G$.

Seja $v \in V(G)$ um vértice qualquer. Para cada floresta $F_{i}$ na floresta-decomposição considerada, temos que $v$ pertence a no máximo uma das componentes de $F_{i}$, e portanto $v$ é um vértice de no máximo $\Upsilon$ árvores. Para cada $\vec{T}$ contendo $v$ como vértice, há apenas um arco saindo de $v$ pois, caso contrário, existiriam dois caminhos orientados de $v$ até a raiz de $\vec{T}$, o que implicaria um circuito no grafo não orientado subjacente a $\vec{T}$, isto é, $T$. Logo, o grau de saída de $v$ é no máximo $\Upsilon$.

Uma orientação acíclica de um grafo é uma orientação que não possui circuitos orientados (a definição de circuito orientado é idêntica à de circuito, exceto pela presença de arcos em vez de arestas). O próximo lema mostra que, a partir de uma orientação limitada, podemos obter uma orientação acíclica, e ainda manter algum limite para o grau de saída dos vértices.

Lema 7.8. Se um grafo $G$ possui uma orientação d-limitada, então $G$ também possui uma orientação $2 d$-limitada acíclica.

Prova. Seja $n$ o número de vértices de $G$. Vamos utilizar indução em $n$. Podemos supor que $n \geq 3$, pois consideramos apenas grafos simples e, assim, o lema vale trivialmente para grafos com até 2 vértices. Como caso base, consideramos $n=3$. Seja, então, $G$ um grafo com 3 vértices e considere uma orientação $d$-limitada de $G$. Se tal orientação for acíclica, não há o que provar. Caso contrário, claramente temos que $G$ é completo e os arcos de $\vec{G}$ formam um circuito orientado de comprimento três. Portanto, esta orientação é 1-limitada. Podemos obter uma orientação 2-limitada acíclica ao invertermos a direção de um arco qualquer de $\vec{G}$.

Para o passo de indução, seja $n \geq 4$ e suponha que o lema vale para grafos de $n-1$ vértices. Seja $G$ um grafo com $n$ vértices, e considere uma orientação $d$-limitada de $G$. Assim:

$$
|E(G)|=\sum_{v \in V(G)} g^{+}(v) \leq d n .
$$

Por outro lado, ao realizarmos contagem de arestas com $G$ não orientado:

$$
\sum_{v \in V(G)} g(v)=2|E(G)| \leq 2 d n .
$$


A última inequação implica que existe algum vértice de $G$ que não sofre mais do que $2 d$ incidências de arestas. Seja $v$ um tal vértice, e seja $H$ o subgrafo de $G$ obtido ao remover-se $v$ (e as arestas que incidem em $v$ ) de $G$. A orientação $d$-limitada de $G$, restrita ao subgrafo $H$, é claramente $d$-limitada e, assim, por hipótese de indução, vale que $H$ possui uma orientação $2 d$-limitada acíclica. Podemos estender tal orientação a uma orientação $2 d$-limitada acíclica de todo o grafo $G$, bastando orientar as arestas de $G$ que não pertencem a $H$ no sentido de saída de $v$. A orientação assim obtida é $2 d$-limitada, já que em $v$ incidem no máximo $2 d$ arestas. Além disso, esta orientação é acíclica, uma vez que em $H$ não há circuitos orientados, e $v$ não pertence a nenhum circuito orientado, por possuir grau de entrada igual a zero.

O lema a seguir é o primeiro resultado nesta seção que relaciona bicliques com orientações acíclicas. Para tanto, é utilizada a noção de ordenação topológica. Considere um grafo orientado $G=(V, A)$. Uma ordenação topológica de $G$ é uma ordenação do seu conjunto de vértices, $V=\left\{v_{1}, \ldots, v_{|V|}\right\}$, tal que, para todo $i \in\{1, \ldots,|V|\}$, o conjunto $\vec{N}\left(v_{i}\right)$ é um subconjunto de $\left\{v_{i+1}, \ldots, v_{|V|}\right\}$.

Lema 7.9. Seja $G=(U, W, E)$ um grafo bipartido que possui uma orientação acíclica. Então, para toda biclique $(A, B)$ de $G$, existe $v \in A \cup B$ tal que $A$ ou $B$ é um subconjunto de $\vec{N}(v)$.

Prova. Considere uma orientação acíclica de $G$. Sejam $V=U \cup W$ e $\left\{v_{1}, \ldots, v_{|V|}\right\}$ uma ordenação topológica de $\vec{G}$. Sejam, ainda, $(A, B)$ uma biclique de $G$, e $v_{i}$ o primeiro vértice de $A \cup B$ que ocorre na ordenação topológica.

Suponha que $v_{i} \in A$. Quando consideramos o grafo não orientado $G$, temos que $v_{i}$ é adjacente a cada $w \in B$. Desta forma, para cada $w \in B$, existe, em $\vec{G}$, o arco $v_{i} w$ ou o arco $w v_{i}$. No entanto, a existência de um arco $w v_{i}$ contrariaria a escolha de $v_{i}$, pois assim, $w$ seria um vértice de $A \cup B$ que ocorreria antes de $v_{i}$ na ordenação topológica. Logo, para cada $w \in B$, temos que $v_{i} w \in A$. Portanto, $B$ é um subconjunto de $\vec{N}\left(v_{i}\right)$.

Suponha que $v_{i} \in B$. Um argumento análogo mostra que para todo vértice $u \in A$ vale que $v_{i} u \in A$, e assim, neste caso, $A$ é um subconjunto de $\vec{N}\left(v_{i}\right)$.

A este ponto estamos aptos a apresentar a cota de Eppstein.

Teorema 7.10 (Cota da arboricidade). Seja $G=(U, W, E)$ um grafo bipartido de $n$ vértices e arboricidade $\Upsilon$. Seja $\mathfrak{B}$ o seu conjunto de bicliques maximais. Então,

$$
|\mathfrak{B}| \leq 2^{2 \Upsilon} \cdot n+2 .
$$

Prova. A utilização dos Lemas 7.7 e 7.8 nos provê uma orientação $2 \Upsilon$-limitada acíclica do grafo $G$. Consideramos $G$ sob tal orientação, e definimos

$$
P_{v}=\mathcal{P}(\vec{N}(v)) \text {, para } v \in V(G) .
$$

Seja $\left\{\left(A_{1}, B_{1}\right),\left(A_{2}, B_{2}\right), \ldots,\left(A_{k}, B_{k}\right)\right\}$ o conjunto de bicliques maximais com arestas de $G$. Pelo Lema 7.9, para cada $i \in\{1, \ldots, k\}$, existe um vértice $v_{i} \in A_{i} \cup B_{i}$ e um subconjunto 
$V_{i} \subseteq \vec{N}\left(v_{i}\right)$ tal que $A_{i}=V_{i}$ ou $B_{i}=V_{i}$. Note que $V_{i} \in P_{v_{i}}$, para todo $i \in\{1,2, \ldots, k\}$ Definimos, ainda:

$$
\begin{aligned}
\mathcal{G} & =\bigcup_{v \in V(G)} P_{v}, \\
\mathcal{F} & =\left\{V_{i} \mid i=1, \ldots, k\right\} .
\end{aligned}
$$

Observe que $\mathcal{F} \subseteq \mathcal{G}$. Agora, queremos mostrar que $|\mathcal{F}|=k$. Para todo $i, j \in\{1,2, \ldots, k\}$, claramente temos que $A_{i} \neq B_{j}$, pois $A_{i}$ e $B_{j}$ são subconjuntos não vazios de $U$ e de $W$, respectivamente. Ademais, como uma biclique maximal fica determinada após ser dada uma de suas coordenadas, vale que $A_{i} \neq A_{j}$ e $B_{i} \neq B_{j}$ para todo $i \neq j$ com $1 \leq i, j \leq k$. Logo, todos os subconjuntos $V_{i}$ dados pelo Lema 7.9 são distintos, e portanto $|\mathcal{F}|=k$.

Como a orientação considerada é $2 \Upsilon$-limitada, temos que $\left|P_{v}\right| \leq 2^{2 \Upsilon}$ para todo $v \in V(G)$. Portanto, $|\mathcal{G}| \leq n 2^{2 \Upsilon}$. Então:

$$
\mathcal{F} \subseteq \mathcal{G} \Rightarrow k=|\mathcal{F}| \leq|\mathcal{G}| \leq n 2^{2 \Upsilon}
$$

Logo, o número de bicliques maximais com arestas de $G$ é limitado superiormente por $n 2^{2 \Upsilon}$. Ao considerarmos as duas possíveis bicliques maximais sem arestas, obtemos o resultado.

\subsection{Cota inferior para o resultado de Eppstein}

É natural a tentativa de se construir famílias de grafos cujo número de bicliques maximais seja próximo de $2^{2 \Upsilon} \cdot n$. Isso significaria que a cota contida no Teorema 7.10 é, em certo sentido, justa. O conteúdo original do Teorema 7.10, descrito na linguagem de [Epp94], é uma cota para o número de subgrafos bipartidos completos maximais de um grafo. No mesmo trabalho em que este resultado foi mostrado, é exposta uma construção, para cada $\Upsilon \in \mathbb{N}$ e cada $n \geq 2 \Upsilon$, de um grafo com arboricidade $\Upsilon, n$ vértices e cujo número de subgrafos bipartidos completos maximais é pelo menos $2^{\Upsilon} n / \Upsilon$.

Vale a pena notar que esta cota inferior, $2^{\Upsilon} n / \Upsilon$, possui uma folga exponencial em $\Upsilon$ frente ao valor $2^{2 \Upsilon} n$. De fato, o problema de se fechar tal folga, ou mostrar uma versão mais forte do resultado referente ao Teorema 7.10 foi um problema deixado em aberto pelo próprio Eppstein naquele trabalho.

O reaproveitamento da família construída por Eppstein poderia ser uma primeira tentativa para a obtenção de uma cota inferior, em função da arboricidade e do número de vértices, para o número de bicliques maximais. Isso, todavia, não é possível, uma vez que os membros desta família são exclusivamente grafos não bipartidos.

Nesta seção, construiremos uma família de dois parâmetros, $\Upsilon$ e $n$, cujos membros são grafos bipartidos de $n$ vértices, arboricidade $\Upsilon$ e com pelo menos $\Omega\left(2^{2 \Upsilon} n / \Upsilon\right)$ bicliques maximais. Esta família também servirá para responder à questão deixada em aberto por Eppstein, acerca da folga exponencial em $\Upsilon$ entre a cota inferior e a cota superior presentes em [Epp94]. De fato, é fácil mostrar que, em um grafo bipartido, o conjunto de bicliques maximais com arestas se injetam naturalmente no conjunto de subgrafos bipartidos completos maximais. Para realizarmos a construção desta família, será necessário o cálculo da arboricidade do grafo coroa, a ser feito no próximo lema. 


\section{Lema 7.11.}

$$
\Upsilon(C G(2 k))=\left\lceil\frac{k(k-1)}{2 k-1}\right\rceil \text {, para todo } k \in \mathbb{N}^{*}
$$

Prova. O conjunto de arestas de $C G(2)$ é vazio, e portanto, por convenção, sua arboricidade é zero. O fato de $C G(4)$ ser acíclico mostra que $\Upsilon(C G(4))=1$. É imediato verificar que tais valores correspondem à expressão do enunciado. Assumimos daqui em diante, portanto, que $k \geq 3$ e consideramos o grafo $G=C G(2 k)$. Vamos mostrar inicialmente que:

$$
\left\lceil\frac{|E(G)|}{|V(G)|-1}\right\rceil=\left\lceil\frac{k(k-1)}{2 k-1}\right\rceil \geq 2
$$

De fato, considere a função quadrática $p(k)=k(k-1)-2 k+1=k^{2}-3 k+1$. Sua concavidade é positiva e sua maior raiz é $\frac{3+\sqrt{5}}{2}<\frac{3+\sqrt{9}}{2}=3$. Logo,

$$
p(k)>0 \Leftrightarrow k(k-1)>2 k-1 \Leftrightarrow\left\lceil\frac{k(k-1)}{2 k-1}\right\rceil \geq 2 .
$$

Sejam $U$ e $W$ as classes de vértices de $G$. Pelo Corolário 7.6, temos que:

$$
\Upsilon(G)=\max _{\substack{H \subseteq G \\|V(H)| \geq 2}}\left\lceil\frac{|E(H)|}{|V(H)|-1}\right\rceil .
$$

Vamos mostrar que o máximo acima é atingido quando $H$ é o subgrafo $H=G$. Para isso, mostraremos que, para todo subgrafo próprio $H$ com pelo menos 2 vértices, existe um subgrafo $J$ tal que $H \subsetneq J \subseteq G$, e que satisfaz

$$
\left\lceil\frac{|E(J)|}{|V(J)|-1}\right\rceil \geq\left\lceil\frac{|E(H)|}{|V(H)|-1}\right\rceil .
$$

Seja, então, $H \subsetneq G$ um subgrafo próprio com $|V(H)| \geq 2$. Sejam $U(H)$ o conjunto $V(H) \cap U$ e $W(H)$ o conjunto $V(H) \cap W$. Definimos $x=|U(H)|, y=|W(H)|$ e $m=|E(H)|$. Note que $m \leq x y$.

Considere o caso em que $\max \{x, y\} \leq 2$. Então, claramente $H$ possui no máximo um circuito, e portanto, $|E(H)| \leq|V(H)|$. Note que, como $|V(H)| \geq 2$, então temos que $|V(H)| \leq 2(|V(H)|-1)$. Assim,

$$
\left\lceil\frac{|E(H)|}{|V(H)|-1}\right\rceil \leq\left\lceil\frac{|V(H)|}{|V(H)|-1}\right\rceil \leq\left\lceil\frac{2(|V(H)|-1)}{|V(H)|-1}\right\rceil=2 .
$$

Portanto, em virtude de (7.3), basta tomarmos $J=G$ para satisfazermos (7.4).

Suponha que $\max \{x, y\} \geq 3$. Sem perda de generalidade, suponha adicionalmente que $\max \{x, y\}=y$. Agora, vamos mostrar que existe um subgrafo $J$ tal que $H \subsetneq J \subseteq G$ e que satisfaz

$$
\frac{|E(J)|}{|V(J)|-1} \geq \frac{|E(H)|}{|V(H)|-1}
$$


Note que isso claramente implica (7.4). Como $H$ é próprio e $x \leq y$, temos que existe um vértice $u^{*} \in U \backslash U(H)$. Definimos o grafo $J$, cujo conjunto de vértices é $V(H) \cup\left\{u^{*}\right\}$, e cujo conjunto de arestas é

$$
E(J)=E(H) \cup\left\{u^{*} w \mid w \in W(H), u w \in E(G)\right\} .
$$

Observe que $J$ possui um vértice a mais, e pelo menos $y-1$ arestas a mais que $H$. Assim, temos que

$$
\begin{aligned}
& \frac{|E(J)|}{|V(J)|-1}-\frac{|E(H)|}{|V(H)|-1} \geq \frac{m+y-1}{x+y}-\frac{m}{x+y-1} \\
& =\frac{(m+y-1)(x+y-1)-m(x+y)}{(x+y)(x+y-1)} \\
& =\frac{y^{2}-2 y-x-m+x y+1}{(x+y)(x+y-1)} \\
& \geq \frac{y^{2}-3 y+1}{(x+y)(x+y-1)} \quad(\text { pois } y \geq x \text { e } x y \geq m) \\
& >0 \text {. (pois } y \geq 3 \text { ) }
\end{aligned}
$$

Portanto, o máximo é atingido em $H=G$. Como $|E(G)|=k(k-1)$ e $|V(G)|=2 k$, o resultado segue.

O próximo teorema descreve a família que serve de cota inferior para o Teorema 7.10, e que também fecha a folga exponencial em $\Upsilon$ entre as cotas inferior e superior de Eppstein, sobre os subgrafos bipartidos completos maximais.

Teorema 7.12. Para todo $\Upsilon \in \mathbb{N}^{*}$ e todo natural $n \geq 4 \Upsilon+2$, existe um grafo bipartido que possui $n$ vértices, arboricidade $\Upsilon$ e cujo número de bicliques maximais com arestas é pelo menos

$$
\left(2^{2 \Upsilon-2}-2\right) \cdot\lfloor n /(4 \Upsilon+2)\rfloor=\Omega\left(2^{2 \Upsilon} \cdot n / \Upsilon\right)
$$

Prova. Sejam $n$ e $\Upsilon$ como no enunciado. Considere a sequência $k \mapsto \frac{k(k-1)}{2 k-1}$. A diferença entre dois elementos sucessivos desta sequência é

$$
\frac{(k+1) k}{2 k+1}-\frac{k(k-1)}{(2 k-1)}=\frac{2 k^{2}}{(2 k-1)(2 k+1)} \leq \frac{k}{2 k-1} \leq 1, \text { para todo } k \in \mathbb{N} .
$$

Assim, podemos tomar $k$ tal que $\Upsilon=\left\lceil\frac{k(k-1)}{2 k-1}\right\rceil \geq\left\lceil\frac{k(k-1)}{2 k}\right\rceil=\left\lceil\frac{k-1}{2}\right\rceil$, e consequentemente, tal que $k-1 \leq 2 \Upsilon$, e, ainda:

$$
2 k \leq 4 \Upsilon+2 \leq n
$$

Por outro lado, observe que

$$
\Upsilon=\left\lceil\frac{k(k-1)}{2 k-1}\right\rceil \leq\left\lceil\frac{k(k-1)}{2(k-1)}\right\rceil \leq \frac{k}{2}+1
$$

o que implica que

$$
k \geq 2 \Upsilon-2 .
$$


Considere o grafo $G$ dado por $\left\lfloor\frac{n}{2 k}\right\rfloor \geq 1$ cópias do grafo coroa $C G(2 k)$. Pelo Lema 7.11, segue que cada componente deste grafo possui arboricidade $\Upsilon$. Além disso, não é difícil observar que uma $\Upsilon$-floresta-decomposição de uma de suas componentes conexas induz uma $\Upsilon$-floresta-decomposição de todo o grafo $G$. Assim, segue que a arboricidade do grafo $G$ é igual a $\Upsilon$. A adição de $n-\left\lfloor\frac{n}{2 k}\right\rfloor$ vértices isolados, a alguma das classes de $G$, claramente não muda sua arboricidade, e $G$ passa a possuir $n$ vértices. Agora, é fácil ver que cada biclique maximal com arestas de uma componente conexa de $G$ também é uma biclique maximal com arestas do grafo $G$. Logo, o número de bicliques maximais com arestas de $G$ é

$$
\left(2^{k}-2\right) \cdot\left\lfloor\frac{n}{2 k}\right\rfloor \geq\left(2^{2 \Upsilon-2}-2\right) \cdot\left\lfloor\frac{n}{4 \Upsilon+2}\right\rfloor=\Omega\left(2^{2 \Upsilon} \cdot \frac{n}{\Upsilon}\right) .
$$

\subsection{A cota do número de arestas}

Alguns autores como Ganter e Wille [GW99] e Kuznetsov [Kuz01] citam mais uma cota superior para o número de bicliques maximais, que é devida a Schütt. Como não temos acesso ao trabalho [Sch87], onde tal resultado é apresentado, nos limitamos aqui a reproduzir a cota relatada por eles.

Teorema 7.13 (Cota do número de arestas). Seja $G=(U, W, E)$ um grafo bipartido com $|E| \geq 3$. Então,

$$
|\mathfrak{B}(G)| \leq\left(\frac{3}{2} \cdot 2^{\sqrt{|E|+1}}\right)-1
$$

\subsection{A cota da convexidade}

Vimos no Teorema 7.12 a construção de uma família de grafos bipartidos para a qual a cota da arboricidade é quase justa. No entanto, esta cota apresenta uma grande folga perante o número de bicliques maximais de algumas famílias, como por exemplo, a família dos grafos $K_{k, k}$. De fato, para qualquer $k$, o grafo $K_{k, k}$ possui apenas uma biclique maximal e, por outro lado, apresenta $k^{2}$ arestas, o que significa que sua arboricidade é pelo menos $k^{2} /(2 k-1)=\Omega(k)$. Assim, a cota da arboricidade, aplicada aos grafos desta família, apresenta grande folga com relação aos números de bicliques maximais. Claramente, as cotas de grau máximo, bem como a cota do número de arestas, fornecem resultado semelhante. Os grafos $K_{k, k}$, no entanto, possuem estruturas muito especiais com relação às suas vizinhanças de vértices. Isto resulta em um argumento simples, a ser apresentado na próxima proposição, que é capaz de limitar o número de bicliques maximais de tais grafos. Tal argumento se baseia em uma operação de contração de vértices. Mais especificamente, seja $G$ um grafo bipartido e $v_{1}, v_{2}$ dois vértices de uma mesma classe. O grafo simples obtido a partir da contração de $v_{1}$ e $v_{2}$ é o grafo obtido após substituir os vértices $v_{1}$ e $v_{2}$ por um novo vértice $v^{*}$, pertencente à mesma classe de vértices de $v_{1}$ e $v_{2}$, e com vizinhança $N\left(v^{*}\right)$ definida por $N\left(v_{1}\right) \cup N\left(v_{2}\right)$. 
Proposição 7.14. Seja $G=(U, W, E)$ um grafo bipartido, e sejam $u_{1}, u_{2} \in U$ vértices com mesmo conjunto de vizinhos, isto é, $N\left(u_{1}\right)=N\left(u_{2}\right)$. Então, o grafo simples obtido a partir da contração dos vértices $u_{1}$ e $u_{2}$ possui o mesmo número de bicliques maximais que $G$.

Prova. Seja $H$ o grafo dado pela contração dos vértices $u_{1}$ e $u_{2}$, e seja $u^{*}$ o vértice de $H$ resultante desta contração. Seja $(A, B)$ uma biclique maximal de $G$. Observe que há apenas duas possibilidades: $u_{1}, u_{2} \in A$ ou $u_{1}, u_{2} \notin A$. De fato, se tivéssemos, por exemplo, $u_{1} \in A$ e $u_{2} \notin A$, teríamos o par $\left(A \cup\left\{u_{2}\right\}, B\right)$ sendo uma biclique pois, como $u_{1} \in A$, temos $u_{1} w \in E$ para todo $w \in B \Rightarrow B \subseteq N\left(u_{1}\right)=N\left(u_{2}\right)$. Assim, se ambos $u_{1}$ e $u_{2}$ não pertencerem a $A$, o par $(A, B)$ também é uma biclique maximal do grafo $H$. Por outro lado, se ambos os vértices pertencerem a $A$, a biclique $(A, B)$ pode ser colocada em correspondência com a biclique maximal $\left(\left(A \backslash\left\{u_{1}, u_{2}\right\}\right) \cup\left\{u^{*}\right\}, B\right)$ do grafo $H$. Esta correspondência é bijetora.

Se aplicarmos iteradamente a Proposição 7.14 para o grafo $K_{k, k}$, concluiremos que os números de bicliques maximais de $K_{1, k}$ e de $K_{k, k}$ são iguais. Como vimos, a utilização da cota da classe mínima provê uma boa delimitação para $\left|\mathfrak{B}\left(K_{1, k}\right)\right|$ e, portanto, indiretamente, também para $\left|\mathfrak{B}\left(K_{k, k}\right)\right|$. No entanto, veremos, em seguida, um exemplo de família para o qual a Proposição 7.14 não é efetiva, e, assim como para $K_{k, k}$, a aplicação das cotas de grau máximo, do número de arestas e da arboricidade resultam apenas em limites exponenciais. Este exemplo servirá de motivação para a Proposição 7.16, a ser exposta em breve, que apresentará uma cota superior quadrática em $n$ para o número de bicliques maximais de grafos que possuam uma determinada propriedade.

Seja $k$ um inteiro qualquer. O grafo Ferrers $(2 k)=(U, W, E)$ é definido da seguinte maneira: $U=\left\{u_{1}, u_{2}, \ldots, u_{k}\right\}, W=\left\{w_{1}, w_{2}, \ldots, w_{k}\right\}$, com $U$ e $W$ disjuntos, e conjunto de arestas definido por $E=\left\{u_{i} w_{j} \in W \mid 1 \leq i \leq k, 1 \leq j \leq i\right\}$. Note que o grau máximo em $U$ (em $W$ ) do grafo assim definido é $k$. Além disso, uma simples observação de que os vértices em $U$ (em $W$ ) possuem graus em uma certa progressão aritmética estabelece que $|E|=k(k+1) / 2$. Logo, assim como observado para o grafo $K_{k, k}$, as cotas da arboricidade, do número de arestas e dos graus máximos, aplicadas a Ferrers $(2 k)$, são exponenciais ${ }^{5} \mathrm{em}$ $k$. Um membro genérico desta família está retratado na Figura 7.6.

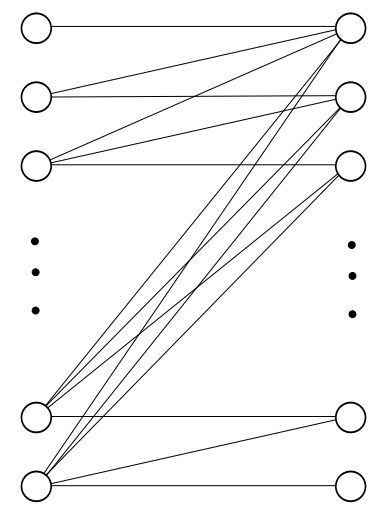

Figura 7.6: Família Ferrers(2k), cujos membros são imunes à Proposição 7.14.

\footnotetext{
${ }^{5}$ Isto vale para toda família densa, e não apenas para Ferrers $(2 k)$ e $K_{k, k}$. De fato, todo grafo com $n$ vértices possui algum vértice com grau pelo menos $2|E| / n$, e a arboricidade é sempre pelo menos $|E| /(n-1)$.
} 
É fácil ver que a Proposição 7.14 não é efetiva para um grafo Ferrers $(2 k)$. De fato, para todo par de vértices distintos $u_{1}, u_{2} \in U$, temos que $N\left(u_{1}\right) \neq N\left(u_{2}\right)$. Além disso, $N\left(w_{1}\right) \neq N\left(w_{2}\right)$, sempre que $w_{1}, w_{2} \in W, w_{1} \neq w_{2}$. Tais propriedades são chamadas de, respectivamente, propriedade de vizinhança distinta em $U$ e propriedade de vizinhança distinta em $W$. Observe que qualquer grafo coroa $C G(2 k)$ também possui ambas tais propriedades. Para grafos que possuam tais propriedades, o argumento de contração de vértices contido na Proposição 7.14 não é efetivo, bem como o argumento da versão dual daquela proposição, sobre contração de vértices em $W$.

A propósito, o argumento da contração de vértices não é o único método de simplificação de grafos que nos permite calcular o número de bicliques maximais a partir daqueles de grafos mais simples. A proposição seguinte, de fácil demonstração, simplifica a contagem no caso de grafos não conexos que, diga-se de passagem, não é o caso dos grafos Ferrers $(2 k)$.

Proposição 7.15. Seja $G$ um grafo bipartido e $G_{1}, G_{2}, \ldots, G_{k}$ suas componentes conexas. $O$ número de bicliques maximais com arestas de $G$ é igual à soma do número de bicliques maximais com arestas de cada $G_{i}$.

Conforme adiantamos, mostraremos uma cota superior para o número de bicliques maximais que, quando aplicada a um grafo $\operatorname{Ferrers}(2 k)$, é quadrática em $k$. Para isto, serão necessárias definições adicionais.

Considere um grafo bipartido $G=(U, W, E)$ com $|W|=k$ e seja $\sigma:[k] \rightarrow W$ uma bijeção. Definimos que um $\sigma$-intervalo de $W$ é um subconjunto de $W$ que é vazio ou da forma $\{\sigma(i), \sigma(i+1), \ldots, \sigma(i+l)\}$ para algum $i \in[k]$ e algum $l \geq 0$. Note que o número de subconjuntos desta forma é igual a $k+(k-1)+\ldots+1=k(k+1) / 2$, e portanto o número de $\sigma$-intervalos de um conjunto $W$ é $[|W|(|W|+1) / 2]+1$. Também note que a intersecção arbitrária de $\sigma$-intervalos é novamente um $\sigma$-intervalo.

Definimos que um vértice $u \in U$ possui vizinhança convexa sob $\sigma$ se o conjunto $N(u)$ for um $\sigma$-intervalo de $W$. Dizemos que $G$ é convexo sobre $W$ se existir uma bijeção $\sigma$ tal que cada $u \in U$ possui vizinhança convexa sob $\sigma$. Como exemplo, estão representados nos dois itens da Figura 7.7 um mesmo grafo bipartido $G=(U, W, E)$, onde $U=\left\{u_{1}, u_{2}, u_{3}\right\}$, $W=\left\{w_{1}, w_{2}, w_{3}, w_{4}, w_{5}\right\}$ e $E$ se encontra explicitamente representado. Considere a bijeção $\sigma$ definida por $\sigma(1)=w_{1}, \sigma(2)=w_{3}, \sigma(3)=w_{4}, \sigma(4)=w_{5}$ e $\sigma(5)=w_{2}$. Tal bijeção está representada implicitamente na Figura 7.7(a) pois, em tal figura, representamos os vértices de $W$, de cima para baixo, na ordem $\sigma(1), \sigma(2), \sigma(3), \sigma(4), \sigma(5)$. Na Figura 7.7(b), vemos representados os vértices de $W$ sob uma ordenação associada a uma outra bijeção, $\tau:\{1,2,3,4,5\} \rightarrow W$, que é idêntica a $\sigma$, a menos dos valores $\tau(4)$ e $\tau(5)$, para os quais $\tau$ associa, respectivamente, os valores $w_{2}$ e $w_{5}$.

Com relação à Figura 7.7(a), pode-se verificar que $u_{1}$ e $u_{2}$ possuem vizinhança convexa sob $\sigma$, enquanto que $u_{3}$ não possui. Na Figura 7.7(b), vê-se que cada vértice $u \in U$ possui vizinhança convexa sob $\tau$, o que mostra que o grafo deste exemplo é convexo sobre $W$. Claramente, para todo $k$, os grafos Ferrers $(2 k)$ e $K_{k, k}$ também são convexos sobre $W$.

A propriedade de ser convexo sobre $U$ pode ser definida analogamente. No entanto, a fim de simplificarmos o que será exposto, convencionamos que, ao dizermos que um grafo é convexo, estamos afirmando que tal grafo é convexo sobre $W$. Adiantamos, no entanto, que é possível que um grafo seja convexo sobre uma das classes, mas não convexo sobre a outra. A Figura 7.8 mostrará um exemplo que, em particular, comprova este fato. 


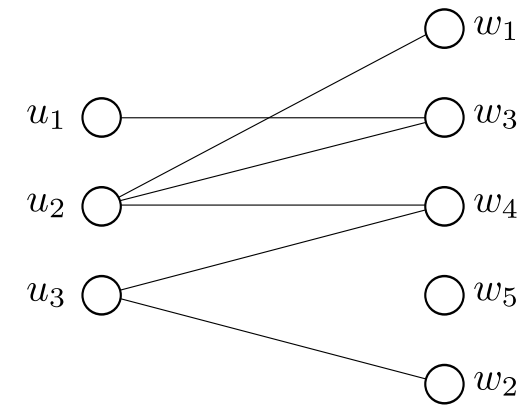

(a) Os vértices $u_{1}$ e $u_{2}$ possuem vizinhança convexa sob $\sigma$.

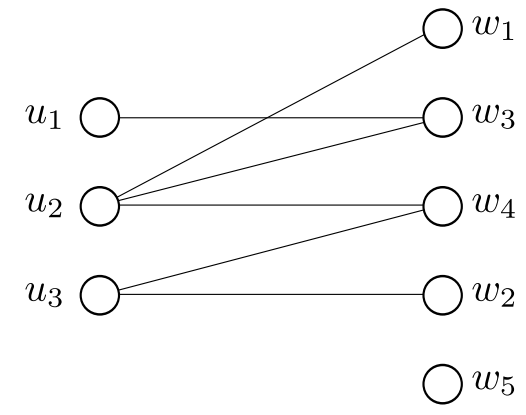

(b) A bijeção $\tau$ torna todo vértice $u \in U$ detentor de vizinhança convexa.

Figura 7.7: Vértices de $U$ com vizinhança convexa sob diferentes bijeções de $W$.

A próxima proposição, cujo conteúdo pode por exemplo ser encontrado em [AAC ${ }^{+}$04], mostra uma cota superior para $|\mathfrak{B}|$ quadrática em $|W|$ para grafos convexos.

Proposição 7.16. Se $G=(U, W, E)$ for um grafo bipartido convexo e $\mathfrak{B}$ seu conjunto de bicliques maximais, então

$$
|\mathfrak{B}| \leq \frac{|W|(|W|+1)}{2}+1=O\left(|W|^{2}\right) .
$$

Prova. Seja $\pi_{2}(\mathfrak{B})$ o conjunto de segundas coordenadas de bicliques maximais de $G$. Recordamos que, pela caracterização de bicliques maximais da Proposição 2.2, temos que $\left|\pi_{2}(\mathfrak{B})\right|=|\mathfrak{B}|$. Seja $k=|W|$ e $\sigma:[k] \rightarrow W$ uma bijeção tal que todo vértice de $U$ possui vizinhança convexa sob $\sigma$. Assim, para todo $u \in U$, o conjunto $N(u)$ é um $\sigma$-intervalo. Vimos na Proposição 5.1 que:

$$
\pi_{2}(\mathfrak{B})=\left\{\bigcap_{u \in I} N(u) \mid I \subseteq U\right\} .
$$

Como a intersecção de um número arbitrário de $\sigma$-intervalos é novamente um $\sigma$-intervalo, temos que a cardinalidade de $\pi_{2}(\mathfrak{B})$ é no máximo o número total de $\sigma$-intervalos, isto é, $\frac{|W|(|W|+1)}{2}+1$.

Uma pergunta natural de ser feita é se a cota da Proposição 7.16 é assintoticamente justa, ou seja, se existem famílias infinitas de grafos bipartidos convexos com $\Omega\left(|W|^{2}\right)$ bicliques maximais. Infelizmente, o grafo Ferrers $(2 k)$ não demonstra isto, já que, como veremos, seu número de bicliques maximais é linear em $k$. O exemplo que será dado no Lema 7.28, no entanto, mostrará que, de fato, esta cota é assintoticamente justa.

Como consequência da Proposição 7.16, podemos exibir um primeiro exemplo de grafo não convexo, a saber, o grafo coroa $C G(6)$. De fato, sabemos que este grafo possui $2^{3}=8$ bicliques maximais, e o valor de $|W|(|W|+1) / 2+1$ para $|W|=3$ é igual a 7 . Este fato pode ser facilmente generalizado, bastando para tanto mostrar que $2^{k}>k(k+1) / 2+1$, para todo $k \geq 3$. Isto significa que, para todo $k \geq 3$, o grafo $C G(2 k)$ não é convexo. O próximo lema mostrará uma generalização desta última afirmação. Note que, para todo $k \geq 2$, o grafo $C G(2 k)$ possui um subgrafo induzido isomorfo a $C G(2(k-1))$. 
Lema 7.17. Todo grafo bipartido que possui um subgrafo induzido isomorfo a $C G(6)$ não é convexo.

Prova. Seja $G=(U, W, E)$ um grafo nessas condições, $k=|W|$, e seja $H$ um subgrafo induzido de $G$ isomorfo a $C G(6)$. Denotamos por $U(H)$ o conjunto $V(H) \cap U$ e por $W(H)$ o conjunto $V(H) \cap W$. Vamos mostrar que, para toda bijeção $\sigma:[k] \rightarrow W$, existe um vértice em $U(H) \subseteq U$ sem a propriedade da vizinhança convexa. Seja $\sigma:[k] \rightarrow W$ uma bijeção. Como $W(H) \subseteq W$, temos que $W(H)=\{\sigma(i), \sigma(j), \sigma(l)\}$, para uma tripla $(i, j, l)$ com $i<j<l$.

Como $H$ é isomorfo a $C G(6)$, temos que existe um vértice em $U(H)$ que, em $H$, é adjacente a $\sigma(i)$ e $\sigma(l)$, mas não adjacente a $\sigma(j)$. Seja $u_{j}$ tal vértice. Observe agora que $u_{j} \sigma(j) \notin E(H) \Rightarrow u_{j} \sigma(j) \notin E(G)$, pois $H$ é subgrafo induzido. Assim, o vértice $u_{j}$ não possui a propriedade da vizinhança convexa, uma vez que $i<j<l$ e o conjunto de vizinhos de $u_{j}$ em $G$ contém os elementos $\sigma(i)$ e $\sigma(l)$ mas não contém o elemento $\sigma(j)$.

A propriedade de um grafo ser ou não convexo pode ser naturalmente generalizada, dando origem a o que chamaremos de convexidade (sobre $W$ ). Sejam $G=(U, W, E)$ um grafo bipartido, $k=|W|$ e $\sigma:[k] \rightarrow W$ uma bijeção. Por definição, a convexidade de $G$ sob $\sigma$ é

$$
\operatorname{conv}(G, \sigma)=\mid\{u \in U \mid u \text { possui vizinhança convexa sob } \sigma\} \mid .
$$

Como exemplo, podemos citar que a convexidade do grafo retratado na Figura 7.7(a), sob aquela bijeção, é igual a dois, e a convexidade do mesmo grafo sob a bijeção representada na Figura 7.7(b) é igual a três. Por fim, definimos a convexidade de $G$ (sobre $W$ ) como sendo

$$
\mathfrak{C}(G)=\max _{\substack{\sigma:[k] \rightarrow W \\ \sigma \text { bijeção }}}\{\operatorname{conv}(G, \sigma)\} .
$$

Note que o valor de $\mathfrak{C}(G)$ é capaz de informar se $G$ é convexo. Mais precisamente, temos que $\mathfrak{C}(G)=|U| \Leftrightarrow G$ é convexo. Conforme detalhamos no início desta seção, os graus máximos, os números de arestas e as arboricidades dos grafos $K_{k, k}$, Ferrers $(2 k)$ e $C G(2 k)$ são muito parecidos, em um sentido que fizemos preciso. A convexidade de tais grafos, no entanto, são muito diferentes. Claramente, $\mathfrak{C}\left(K_{k, k}\right)=\mathfrak{C}(F \operatorname{errer} s(2 k))=k$, pois tais grafos são convexos. Por outro lado, não é difícil mostrar que $\mathfrak{C}(C G(2 k)) \leq 2$, para todo $k \in \mathbb{N}$. De fato, o grafo coroa $C G(2 k)$ possui a propriedade da vizinhança distinta em $U$, e cada $N(u)$ é um conjunto de cardinalidade $k-1$. Além disso, para qualquer bijeção $\sigma$, existem apenas dois $\sigma$-intervalos de cardinalidade $k-1$, a saber, $\{\sigma(1), \sigma(2), \ldots, \sigma(k-1)\}$ e $\{\sigma(2), \sigma(3), \ldots, \sigma(k)\}$, o que prova que $\mathfrak{C}(G) \leq 2$.

É claro que a convexidade sobre $U$ pode ser definida analogamente, assim como no caso da propriedade. Além disso, as convexidades de um grafo bipartido sobre cada uma de suas classes podem ser muito diferentes, como veremos na Figura 7.8. No item a) daquela figura, podemos ver claramente que o grafo retratado é convexo sobre $W$. Com relação à convexidade sobre $U$, note primeiramente que cada um dos vértices $w_{i}, i \in\{1,2,3\}$ é adjacente a $u^{*}$ e a exatamente um vértice a mais, a saber, $u_{i}$. Considere uma bijeção qualquer $\sigma:\{1,2,3,4\} \rightarrow U$, e seja $j$ tal que $j=\sigma^{-1}\left(u^{*}\right) \Leftrightarrow \sigma(j)=u^{*}$. É fácil ver que um vértice $w_{i}$ possui vizinhança convexa sob $\sigma$ se e só se $\sigma(j-1)=u_{i}$ ou $\sigma(j+1)=u_{i}$. Assim, a convexidade sobre $U$ daquele grafo é no máximo dois. Naquela figura, a bijeção implícita 
na ordenação de $U$ é facilmente verificada como apresentando convexidade dois, e portanto, a convexidade daquele grafo sobre $U$ é igual a dois. O item b) da Figura 7.8 mostra que podemos adicionar um vértice $w_{4}$ de maneira a manter a propriedade do grafo ser convexo sobre $W$, enquanto que a convexidade sobre $U$ permanece sendo igual a dois. Claramente, um número arbitrário de vértices pode ser adicionado desta maneira a $W$.

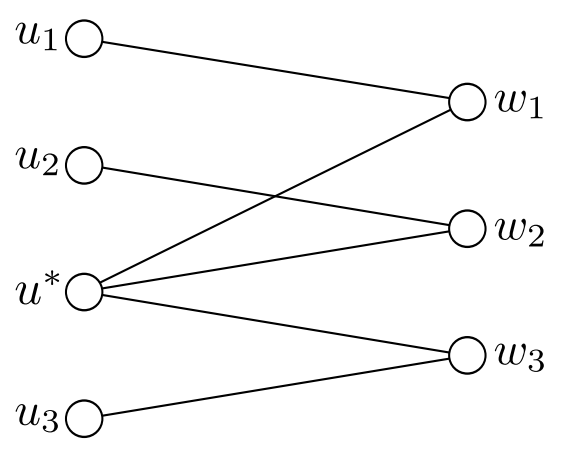

(a)

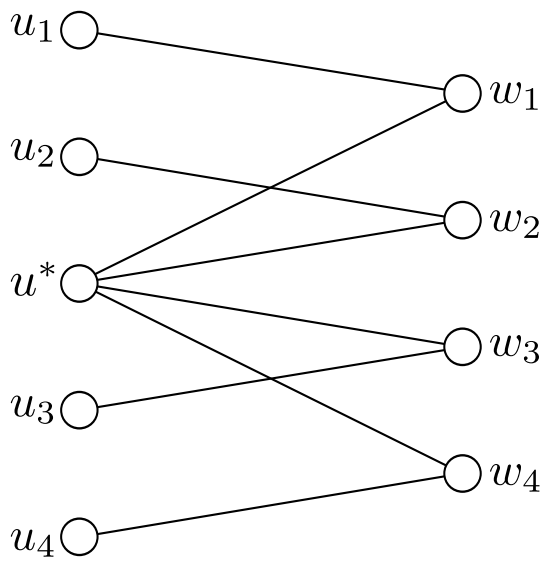

(b)

Figura 7.8: Diferença arbitrária entre a convexidade sobre $W$ e sobre $U$.

Assim como no caso da propriedade, diremos apenas convexidade de $G$ para nos referirmos à convexidade de $G$ sobre $W$. Na Seção 7.10, abordaremos a questão da complexidade computacional do cálculo desta medida.

Como vimos na Proposição 7.16, famílias de grafos convexos possuem um número quadrático de bicliques maximais e, assim, é natural imaginar a existência de cotas superiores melhores do que as já apresentadas para grafos com convexidade "suficientemente grande". Vamos mostrar que esta suposição é verdadeira. Antes, porém, exporemos um resultado de decomposição, que permite delimitar o número de bicliques maximais de um grafo em função do número de bicliques maximais de dois de seus subgrafos.

O próximo teorema apresenta tal resultado de decomposição. Diferentemente da apresentação original, realizada por Wille em [Wil82] sob linguagem algébrica, exporemos tal resultado com o emprego da linguagem de teoria dos grafos.

Teorema 7.18. Seja $G=(U, W, E)$ um grafo bipartido e seja $U=U_{1} \cup U_{2}$. O número de bicliques maximais de $G$ é no máximo o produto do número de bicliques maximais de $G_{1}=G\left[U_{1} \cup W\right]$ e $G_{2}=G\left[U_{2} \cup W\right]$. Esta cota é justa.

Prova. Seja $\mathfrak{B}$ o conjunto de bicliques maximais de $G$, e sejam $\mathfrak{B}_{1}$ e $\mathfrak{B}_{2}$ os conjuntos de bicliques maximais de $G_{1}$ e $G_{2}$, respectivamente. Dados $u \in U, w \in W$, sejam $N(u)$ e $N(w)$ os conjuntos de vizinhos de vértices do grafo $(U, W, E)$, conforme definido em 7.1. Analogamente, definimos como $N_{1}(u), N_{1}(w)$ e $N_{2}(u), N_{2}(w)$ os conjuntos de vizinhos dos vértices de cada classe de vértices dos grafos $G_{1}$ e $G_{2}$. É fácil observar que $N_{1}(u)=N(u)$ 
para todo $u \in U_{1}$ e $N_{2}(u)=N(u)$ para todo $u \in U_{2}$. Também definimos:

$$
\begin{aligned}
\triangleright: \mathcal{P}(U) & \rightarrow \mathcal{P}(W) & \triangleleft: \mathcal{P}(W) & \rightarrow \mathcal{P}(U) \\
A & \mapsto \bigcap_{u \in A} N(u) & B & \mapsto \bigcap_{w \in B} N(w) .
\end{aligned}
$$

Assim como, para $i \in\{1,2\}$ :

$$
\begin{aligned}
\triangleright_{i}: \mathcal{P}\left(U_{i}\right) & \rightarrow \mathcal{P}(W) & \triangleleft_{i}: \mathcal{P}(W) & \rightarrow \mathcal{P}\left(U_{i}\right) \\
A & \mapsto \bigcap_{u \in A} N_{i}(u) & B & \mapsto \bigcap_{w \in B} N_{i}(w) .
\end{aligned}
$$

Isto é, as funções ${ }^{\triangleright} \mathrm{e}^{\triangleleft}$ correspondem aos operadores de derivação do contexto induzido por $(U, W, E)$, assim como os dois pares de funções ${ }^{\triangleright_{1}},{ }^{\triangleleft_{1}} \mathrm{e}^{\triangleright_{2}}, \triangleleft_{2}$ correspondem aos operadores de derivação dos subcontextos induzidos pelos grafos $G_{1}$ e $G_{2}$. Agora, definiremos a seguinte função $\varphi: \mathfrak{B} \rightarrow \mathfrak{B}_{1} \times \mathfrak{B}_{2}$ :

$$
\varphi((A, B))=\left(\left(A \cap U_{1},\left(A \cap U_{1}\right)^{\triangleright}\right),\left(A \cap U_{2},\left(A \cap U_{2}\right)^{\triangleright}\right)\right) .
$$

A função $\varphi$ está bem definida. De fato, seja $(A, B) \in \mathfrak{B}$. Então, $A \cap U_{1}$ é o conjunto de vértices de $G_{1}$ adjacentes a cada $b \in B$, ou seja, $A \cap U_{1}$ está na imagem da função ${ }^{{ }_{1}}$. Logo, $A \cap U_{1}$ é a primeira coordenada de uma biclique maximal de $G_{1}$, pelas Proposições 2.2 e 2.3, item (iii). Consequentemente, $\left(A \cap U_{1},\left(A \cap U_{1}\right)^{\triangleright}\right)$ é uma biclique maximal de $G_{1}$. Analogamente, $\left(A \cap U_{2},\left(A \cap U_{2}\right)^{\triangleright}\right)$ é uma biclique maximal de $G_{2}$.

Vamos agora mostrar que $\varphi$ é injetora. Suponha que $\left(A_{1}, B_{1}\right)$ e $\left(A_{2}, B_{2}\right)$ são tais que $\varphi\left(\left(A_{1}, B_{1}\right)\right)=\varphi\left(\left(A_{2}, B_{2}\right)\right)$. Então $A_{1} \cap U_{1}=A_{2} \cap U_{1}$ e $A_{1} \cap U_{2}=A_{2} \cap U_{2}$. Como $U_{1} \cup U_{2}=U$, temos que $A_{1}=A_{2}$. Consequentemente, $B_{1}=B_{2}$, e portanto $\varphi$ é injetora.

No caso em que $G=(U, W, E)$ é isomorfo ao grafo coroa, e $\left\{U_{1}, U_{2}\right\}$ é uma partição qualquer de $U$, não é difícil mostrar que ${ }_{1}^{\triangleright} \mathrm{e}_{2}^{\triangleright}$ são injetoras. Assim, os números de segundas coordenadas de bicliques maximais de $G_{1}$ e de $G_{2}$ são, respectivamente, $2^{\left|U_{1}\right|}$ e $2^{\left|U_{2}\right|}$, e cujo produto é igual a $2^{\left|U_{1}\right|+\left|U_{2}\right|}=2^{|U|}=|\mathfrak{B}(G)|$.

Como vimos na Proposição 7.16, grafos convexos possuem um número de bicliques maximais quadrático em $|W|$. A condição de convexidade porém, pode ser muito restritiva, já que qualquer grafo com um subgrafo induzido isomorfo a $C G(6)$ não é convexo, conforme o Lema 7.17. O Teorema 7.19 generaliza o resultado da Proposição 7.16, e é particularmente útil quando $|U|-\mathfrak{C}$ é pequeno relativamente a $|U|$, mas é aproveitável mesmo quando $|U|-\mathfrak{C}$ é uma fração arbitrariamente grande de $|U|$, como veremos no Teorema 7.23.

Teorema 7.19 (Cota da convexidade). Seja $G=(U, W, E)$ um grafo bipartido de convexidade $\mathfrak{C}$. Então,

$$
|\mathfrak{B}(G)| \leq 2^{|U|-\mathfrak{C}}\left(\frac{|W|(|W|+1)}{2}+1\right)=O\left(2^{|U|-\mathfrak{C}}|W|^{2}\right) .
$$

Prova. Sejam $k=|W|$ e $\sigma:[k] \rightarrow W$ uma bijeção que maximize $\operatorname{conv}(G, \sigma)$. Definimos os conjuntos

$$
\begin{aligned}
& U_{1}=\{u \in U \mid u \text { possui vizinhança convexa sob } \sigma\} \mathrm{e} \\
& U_{2}=U \backslash U_{1} .
\end{aligned}
$$


Considere os subgrafos induzidos de $G$ definidos por:

$$
\begin{aligned}
& G_{1}=G\left[U_{1} \cup W\right] \mathrm{e} \\
& G_{2}=G\left[U_{2} \cup W\right] .
\end{aligned}
$$

Note que $G_{1}$ é convexo. O Teorema 7.18 afirma que $|\mathfrak{B}(G)| \leq\left|\mathfrak{B}\left(G_{1}\right)\right| \cdot\left|\mathfrak{B}\left(G_{2}\right)\right|$. Ao utilizarmos a Proposição 7.16 para obtermos uma cota superior quadrática para $\left|\mathfrak{B}\left(G_{1}\right)\right|$, e utilizarmos a cota da classe mínima para $\left|\mathfrak{B}\left(G_{2}\right)\right|$, temos o resultado.

É natural que nos perguntemos se existem grafos para os quais a cota da convexidade é útil a ponto de prover cotas superiores para $|\mathfrak{B}|$ mais justas do que as cotas obtidas pelos demais métodos conhecidos. Mostraremos afirmativamente esta suposição e, para isso, consideraremos grafos cujas classes podem ser biparticionadas de maneira que seus vértices pertençam a um subgrafo induzido Ferrers ou a um subgrafo induzido coroa. Mais especificamente, definimos que um grafo bipartido $(U, W, E)$ é Ferrers-coroa se $U$ e $W$ admitem bipartições, respectivamente, $\left\{U_{F}, U_{C}\right\}$ e $\left\{W_{F}, W_{C}\right\}$ tais que $\left|U_{F}\right|=\left|W_{F}\right|,\left|U_{C}\right|=\left|W_{C}\right|$ de maneira que $G\left[U_{F} \cup W_{F}\right]$ é isomorfo ao grafo Ferrers $\left(\left|U_{F}\right|+\left|W_{F}\right|\right)$ e $G\left[U_{C} \cup W_{C}\right]$ é isomorfo ao grafo $C G\left(\left|U_{C}\right|+\left|W_{C}\right|\right)$. Uma maneira simples de se construir um grafo Ferrers-coroa é tomando a união disjunta entre um grafo Ferrers $(2 f)$ e um grafo $C G(2 c)$, com $f$ e $c$ quaisquer. Observe que o grafo assim obtido possui ambas as propriedades das vizinhanças distintas, mas que no entanto, não é conexo. Um exemplo de grafo Ferrers-coroa conexo e com ambas as propriedades de vizinhanças distintas está representado na Figura 7.9.

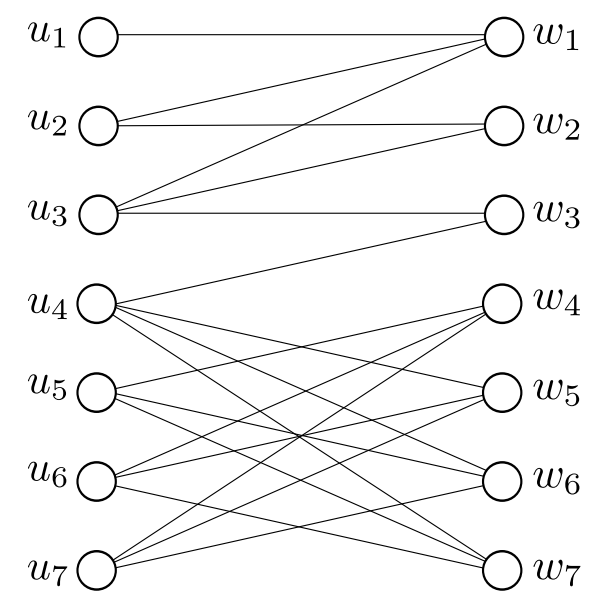

Figura 7.9: Um grafo Ferrers-coroa conexo, com vizinhanças distintas, $\left|U_{F}\right|=3\left(=\left|W_{F}\right|\right)$ e $\left|U_{c}\right|=4\left(=\left|W_{C}\right|\right)$.

O grafo da Figura 7.9 é um caso particular da seguinte construção: toma-se uma união disjunta entre um grafo Ferrers e um grafo coroa, e se adiciona arbitrariamente arestas entre vértices de $U_{C}$ e $W_{F}$. Esta adição de arestas mantém ambas as propriedades de vizinhanças distintas, uma vez que para quaisquer dois vértices $u_{1}, u_{2}$ de $U_{C}$, os conjuntos $N\left(u_{1}\right) \cap W_{C}$ e $N\left(u_{2}\right) \cap W_{C}$ não são iguais. Para vértices $u_{1}, u_{2} \in U_{F}$ ou $u_{1} \in U_{F}, u_{2} \in U_{C}$, a desigualdade $N\left(u_{1}\right) \neq N\left(u_{2}\right)$ é clara. Note que, desde que $\left|U_{C}\right| \geq 3$, esta operação também garante que o grafo assim obtido é conexo. Também note que, se adicionarmos arestas entre vértices de 
$U_{F}$ e $W_{C}$, em vez de entre vértices de $U_{C}$ e $W_{F}$, também obtemos um grafo conexo e com ambas as propriedades.

A próxima proposição nos auxiliará a medir a performance de todas as cotas, a menos da cota da convexidade, para grafos Ferrers-coroas.

Proposição 7.20. Seja $G$ um grafo Ferrers-coroa de $2 k$ vértices. Então, o grau máximo dos vértices de $G$, sobre cada uma das classes de vértices, é pelo menos $\lceil(k-1) / 2\rceil$. Além disso, a arboricidade de $G$ é pelo menos $\lceil k / 6\rceil$ e seu número de arestas pelo menos $\left\lceil k^{2} / 3-3 / 8\right\rceil$.

Prova. Sejam $\left\{U_{C}, U_{F}\right\}$ e $\left\{W_{C}, W_{F}\right\}$ as bipartições de $U$ e $W$ tais que $G\left[U_{F} \cup W_{F}\right]$ é isomorfo a Ferrers $(2 f)$ e $G\left[U_{C} \cup W_{C}\right]$ é isomorfo a $C G(2 c)$, onde $f=\left|U_{F}\right|$ e $c=\left|U_{C}\right|$. Como $G$ possui $2 k$ vértices, vale que $k=f+c$.

Com relação ao grau máximo sobre a classe $U$ de $G$, note que existe um vértice $u \in U_{F}$ com grau pelo menos igual a $f$, e todo vértice de $U_{C}$ apresenta grau pelo menos igual a $c-1=k-f-1$. Seja $u^{*}$ um vértice qualquer de $U_{C}$. A soma dos graus de $u$ e $u^{*}$ é pelo menos $(k-1)$, e portanto, um deles apresenta grau maior ou igual a $\lceil(k-1) / 2\rceil$. Um argumento análogo estabelece o mesmo limitante inferior para o grau máximo sobre os vértices de $W$ do grafo $G$.

Para limitarmos inferiormente $\Upsilon(G)$, escolhemos os seguintes subgrafos:

$$
H_{F}=G\left[U_{F} \cup W_{F}\right] \quad \text { e } \quad H_{C}=G\left[U_{C} \cup W_{C}\right],
$$

e utilizamos o Corolário 7.6. As frações presentes naquele corolário, correspondentes a $H_{F} \mathrm{e}$ $H_{C}$ são:

$$
\begin{aligned}
& \frac{\left|E\left(H_{F}\right)\right|}{\left|V\left(H_{F}\right)\right|-1}=\frac{f(f+1)}{2} \cdot \frac{1}{2 f-1}=\frac{f(f+1)}{4 f-2} \geq \frac{f+1}{4}=\frac{k-c+1}{4} \quad \mathrm{e} \\
& \frac{\left|E\left(H_{C}\right)\right|}{\left|V\left(H_{C}\right)\right|-1}=\frac{c(c-1)}{2 c-1} \geq \frac{c-1}{2} .
\end{aligned}
$$

E, portanto,

$$
\Upsilon(G) \geq \max \left\{\frac{k-c+1}{4}, \frac{c-1}{2}\right\} \geq \min _{0 \leq c \leq k} \max \left\{\frac{k-c+1}{4}, \frac{c-1}{2}\right\},
$$

ou seja, $\Upsilon(G)$ está limitado inferiormente pelo mínimo do máximo entre duas funções lineares em c. Claramente, este mínimo é atingido quando $\frac{1}{4}(k-c+1)=\frac{1}{2}(c-1)$, ou seja, quando $c=\frac{k+3}{3}$, atingindo o valor $\frac{k}{6}$. Como $\Upsilon(G)$ é inteiro, segue que $\Upsilon(G) \geq\left\lceil\frac{k}{6}\right\rceil$.

Com relação ao número de arestas, temos claramente que

$$
\begin{aligned}
|E| \geq\left|E\left(H_{F}\right)\right|+\left|E\left(H_{C}\right)\right| & =\frac{f(f+1)}{2}+c(c-1) \\
& =\frac{f(f+1)}{2}+(k-f)(k-f-1) \\
& =\frac{f^{2}}{2}+\frac{f}{2}+k^{2}-2 k f-k+f^{2}+f \\
& =\frac{3 f^{2}}{2}+\left(\frac{3}{2}-2 k\right) f+k^{2}-k .
\end{aligned}
$$


Assim como

$$
\frac{3 f^{2}}{2}+\left(\frac{3}{2}-2 k\right) f+k^{2}-k \geq \min _{0 \leq f \leq k}\left\{\frac{3 f^{2}}{2}+\left(\frac{3}{2}-2 k\right) f+k^{2}-k\right\} .
$$

Considere a função quadrática em $f$ associada à expressão com o mínimo aplicado acima. Tal função apresenta concavidade positiva, e portanto, atinge mínimo para $f$ igual a

$$
\left(2 k-\frac{3}{2}\right) \cdot \frac{1}{3}=\frac{2 k}{3}-\frac{1}{2}
$$

e o valor deste mínimo é

$$
\frac{3}{2}\left(\frac{2 k}{3}-\frac{1}{2}\right)^{2}+\left(\frac{3}{2}-2 k\right)\left(\frac{2 k}{3}-\frac{1}{2}\right)+k^{2}-k=\frac{k^{2}}{3}-\frac{3}{8}
$$

Sendo $|E|$ um inteiro, segue que $|E| \geq\left\lceil\frac{k^{2}}{3}-\frac{3}{8}\right\rceil$.

Considere uma família $\mathcal{F}=\left\{G_{i}\right\}_{i \in \mathbb{N}^{*}}$ de grafos Ferrers-coroa tal que, para cada $i$, a razão entre a convexidade de $G_{i}$ e a cardinalidade de $U,|U(i)|$, seja estritamente maior que dois terços. Uma maneira simples de se obter uma tal família é tomar grafos Ferrers-coroa com bipartição de $U$ sendo $\left\{U_{F}, U_{C}\right\}$ tal que $\left|U_{F}\right| \geq(2 / 3+\epsilon)|U|$, com $\epsilon>0$ fixado, e adicionar apenas arestas entre $U_{C}$ e $W_{F}$. O próximo teorema mostra que, para qualquer família $\mathcal{F}$ satisfazendo esta condição, a cota da convexidade provê, assintoticamente, melhores resultados que as demais cotas. Será mostrado ainda no Teorema 7.21 que, se a convexidade de cada grafo da família atender a uma exigência assintoticamente mais forte, a saber, de ser pelo menos $\left(1-\log _{2}|U|\right) \cdot|U|$, então a cota da convexidade estabelece que os grafos de tal família possuem um número cúbico de bicliques maximais, enquanto que os outros resultados fornecem apenas limites superiores exponenciais.

Teorema 7.21. Seja $\mathcal{F}=\left\{G_{i}\right\}_{i \in \mathbb{N}^{*}}$ uma família de grafos Ferrers-coroa e seja $k(i)$ a cardinalidade das classes de vértices de cada $G_{i}$. Então, as cotas da classe minima, do grau máximo, da arboricidade e de número de arestas, quando aplicadas aos grafos de $\mathcal{F}$, são limitadas inferiormente por funções exponenciais em $k(i)$. Adicionalmente, suponha que a sequência $(k(1), k(2), \ldots)$ seja não-decrescente e ilimitada. Assim,

- Se existir $\epsilon>0$ tal que $\mathfrak{C}\left(G_{i}\right) \geq\left(\frac{2}{3}+\epsilon\right) k(i)$ para todo $i \in \mathbb{N}^{*}$, então existe $i_{0} \in \mathbb{N}^{*}$ tal que a cota da convexidade é mais justa que as cotas da classe mínima, dos graus máximos, da arboricidade e de número de arestas para todo grafo $G_{i}$ com $i \geq i_{0}$.

- Se existir $\alpha \in \mathbb{N}$ tal que $\mathfrak{C}\left(G_{i}\right) \geq k(i)-\alpha \cdot \log _{2} k(i)$ para todo $i \in \mathbb{N}^{*}$, então a cota da convexidade é limitada superiormente por $\frac{1}{2}\left[k(i)^{\alpha+2}+k(i)^{\alpha+1}+2 k(i)^{\alpha}\right]$. Em particular, se $\mathfrak{C}\left(G_{i}\right) \geq k(i)-\log _{2} k(i)$ para todo $i \in \mathbb{N}^{*}$, então a cota da convexidade é limitada superiormente por uma função polinomial cúbica em $k(i)$.

Prova. Os fatores exponenciais das cotas da classe mínima, dos graus máximos, da arboricidade e de número de arestas são, respectivamente, $2^{\min \{|U|,|W|\}}, 2^{\Delta_{U}}, 2^{\Delta_{W}}, 2^{2 \Upsilon}$ e $2^{\sqrt{|E|+1}}$. A limitação inferior exponencial para a cota da classe mínima é clara, uma vez que um 
grafo Ferrers-coroa sempre possui classes de vértices de mesma cardinalidade, enquanto que a mesma afirmação sobre as outras cotas segue diretamente da Proposição 7.20, que limita inferiormente os valores de $\Delta_{U}, \Delta_{W}, \Upsilon$ e $|E|$ para qualquer grafo Ferrers-coroa.

Suponha o caso em que existe $\epsilon>0$ tal que $\mathfrak{C}\left(G_{i}\right) \geq\left(\frac{2}{3}+\epsilon\right) k(i)$ para todo $i$. Então o expoente presente no fator exponencial da cota da convexidade pode ser limitado superiormente:

$$
k(i)-\mathfrak{C}\left(G_{i}\right) \leq\left(\frac{1}{3}-\epsilon\right) k(i) .
$$

Por outro lado, pela Proposição 7.20, temos que

$$
\begin{aligned}
\min \left\{\Delta_{U}\left(G_{i}\right), \Delta_{W}\left(G_{i}\right)\right\} & \geq \frac{(k(i)-1)}{2} \\
2 \Upsilon\left(G_{i}\right) & \geq \frac{k(i)}{3} \\
\sqrt{\left|E\left(G_{i}\right)\right|+1} & >\sqrt{\frac{k(i)^{2}}{3}}=\frac{\sqrt{3}}{3} k(i) .
\end{aligned}
$$

e cada uma das funções lineares em $k(i)$, mostradas como limitantes inferiores acima, apresenta coeficiente angular estritamente maior que $(1 / 3-\epsilon)$.

Agora, suponha que existe $\alpha \in \mathbb{N}$ tal que $\mathfrak{C}\left(G_{i}\right) \geq k(i)-\alpha \log _{2} k(i)$, para todo $i$. Neste caso, vale que $k(i)-\mathfrak{C}\left(G_{i}\right) \leq \alpha \cdot \log _{2} k(i)$, o que implica que

$$
\begin{aligned}
2^{k(i)-\mathfrak{C}\left(G_{i}\right)}\left(\frac{k(i)(k(i)+1)}{2}+1\right) & \leq k(i)^{\alpha}\left(\frac{k(i)(k(i)+1)}{2}+1\right) \\
& =\frac{1}{2} \cdot\left[k(i)^{\alpha+2}+k(i)^{\alpha+1}+2 k(i)^{\alpha}\right] .
\end{aligned}
$$

O Teorema 7.21 mostra que a cota da convexidade dá, assintoticamente, resultados mais justos que os obtidos pelas outras cotas, quando aplicada a qualquer família infinita de grafos Ferrers-coroa que satisfaçam uma exigência de convexidade mínima. No entanto, esta exigência de convexidade é de pelo menos $(2 / 3+\epsilon)|U|$. No que segue, construiremos uma família de grafos Ferrers-coroa com dois parâmetros, $c$ e $k$, com $0<c \leq 1$, tal que, para cada $c$ fixado (mesmo inferiores a 2/3), os grafos da subfamília $\left\{G_{c, k}\right\}_{k \in \mathbb{N}^{*}}$ possuem $k$ vértices na classe $U($ e $W)$, convexidade exatamente $\lfloor c k\rfloor$ e, ainda assim, nesta subfamília, a cota da convexidade é assintoticamente mais justa que as demais cotas, isto é, mais justa que as demais cotas para todo $k$ suficientemente grande.

Antes de definirmos tal família, precisaremos considerar uma família baseada na construção do grafo da Figura 7.9. Naquela ocasião, tomamos cópias disjuntas de um grafo Ferrers $(2 f)$ e um grafo $C G(2 r)$, adicionamos arestas entre vértices de $U_{C}$ e $W_{F}$, e argumentamos que o grafo resultante era conexo e possuia ambas as propriedades das vizinhanças distintas. É possível observar sem muitas dificuldades que, se, em vez de adicionarmos arbitrariamente arestas entre $U_{C}$ e $W_{F}$, realizarmos a adição de todas as possíveis arestas que possuam pontas em $U_{C}$ e $W_{F}$ ou pontas em $U_{F}$ e $W_{C}$, teremos igualmente um grafo que possui todas as propriedades mencionadas. 
Sejam $r \geq 1$ e $f \geq 1$ dois inteiros. Definimos que o grafo Ferrers-coroa completo de $(r, f)$ é $C F C G(r, f)=(U, W, E)$, onde:

$$
\begin{aligned}
U_{C} & =\left\{u_{1}, u_{2}, \ldots, u_{r}\right\}, \quad U_{F}=\left\{u_{r+1}, u_{r+2}, \ldots, u_{r+f}\right\} \\
W_{C} & =\left\{w_{1}, w_{2}, \ldots, w_{r}\right\}, W_{F}=\left\{w_{r+1}, w_{r+2}, \ldots, w_{r+f}\right\} \\
U & =U_{C} \cup U_{F} \\
W & =W_{C} \cup W_{F} \\
E_{C} & =\left\{u_{i} w_{j} \mid 1 \leq i \leq r, 1 \leq j \leq r, i \neq j\right\} \\
E_{F} & =\left\{u_{i} w_{j} \mid r+1 \leq i \leq r+f, r+1 \leq j \leq i\right\} \\
E & =E_{F} \cup E_{C} \cup\left\{u_{i} w_{j} \mid u_{i} \in U_{F}, w_{j} \in W_{C}\right\} \cup\left\{u_{i} w_{j} \mid u_{i} \in U_{C}, w_{j} \in W_{F}\right\} .
\end{aligned}
$$

Note que os subgrafos induzidos $G\left[U_{C} \cup W_{C}\right]$ e $G\left[U_{F} \cup W_{F}\right]$ são isomorfos, respectivamente, a $C G(2 r)$ e a Ferrers $(2 f)$. Também note que os argumentos das Proposições 7.14 e 7.15, acerca de contrações e contagem por componentes conexas, não se aplicam a quaisquer grafos $C F C G(r, f)$. Por fim, observe que cada um dos vértices $u_{r+f}$ e $w_{r+1}$ possuem grau igual a $r+f$. O grafo $C F C G(3,2)$ está retratado na Figura 7.10. Naquela figura, arestas de $E_{C}$ e de $E_{F}$ estão representadas com traço cheio, enquanto que as demais estão em pontilhado.

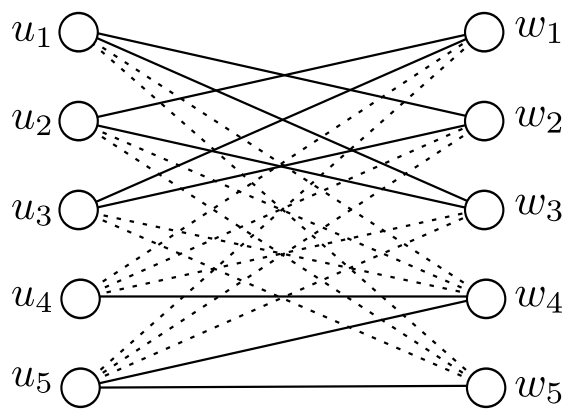

Figura 7.10: Grafo Ferrers-coroa completo com $r=3$ e $f=2$.

A família que mencionamos possuir como parâmetros $c$ e $k$ com $0 \leq c<1$, é essencialmente a família $C F C G(r, f)$ com o parâmetro $r$ sendo fixado em função de $f$. Definiremos tal família após mostrada a seguinte proposição, que efetua o cálculo da convexidade dos grafos Ferrers-coroa completos.

\section{Proposição 7.22.}

$$
\mathfrak{C}(C F C G(r, f))=f+1 \text {, para todo } r, f \in \mathbb{N}^{*} \text { com } f \geq 2 .
$$

Prova. Sejam $U$ e $W$ as classes de vértices de $C F C G(r, f)$ e $U_{F}, U_{C}, W_{F}, W_{C}$ os subconjuntos de $U$ e de $W$ conforme realizado durante sua construção. Note que $r=\left|U_{C}\right|$ e que $f=\left|U_{F}\right|$. Seja $k=|W|=r+f$. Considere a bijeção $\sigma:[k] \rightarrow W$ dada por $\sigma(i)=w_{i}$. O grafo $C F C G(r, f)$ apresenta convexidade $f+1$ sob tal bijeção, já que cada vértice de $U_{F}$ possui vizinhança convexa sob $\sigma$, assim como o vértice $u_{1}$. Isto mostra que $\mathfrak{C}(C F C G(r, f)) \geq f+1$.

Vamos mostrar que $\mathfrak{C}(C F C G(r, f)) \leq f+1$, da seguinte maneira. Será mostrado que no máximo dois vértices de $U_{C}$ possuem a propriedade da vizinhança convexa, para qualquer 
bijeção de $[k]$ em $W$. Depois, será mostrado que se uma bijeção $\sigma:[k] \rightarrow W$ deixa dois vértices de $U_{C}$ com a propriedade da vizinhança convexa, então existe algum vértice de $U_{F}$ sem a propriedade da vizinhança convexa.

Para o primeiro fato, note primeiramente que, quaisquer dois vértices distintos de $U_{C}$ possuem vizinhanças distintas, e tais vizinhanças possuem cardinalidade $k-1$. Ademais, qualquer bijeção $\sigma:[k] \rightarrow W$ possui exatamente dois $\sigma$-intervalos de cardinalidade $k-1$, a saber, $\{\sigma(1), \sigma(2), \ldots, \sigma(k-1)\}$ e $\{\sigma(2), \sigma(3), \ldots, \sigma(k)\}$.

Com relação ao segundo fato, seja $\sigma:[k] \rightarrow W$ uma bijeção e sejam $u_{i}, u_{j} \in U_{C}$ vértices com a propriedade da vizinhança convexa sob $\sigma$. Observe que $u_{i}, u_{j} \in U_{C} \Rightarrow 1 \leq i, j \leq r$. Agora, note que, como $u_{i}$ e $u_{j}$ possuem vizinhança convexa sob $\sigma$, e ainda, $N\left(u_{i}\right)=W \backslash\left\{w_{i}\right\}$ e $N\left(u_{j}\right)=W \backslash\left\{w_{j}\right\}$, então claramente temos que $\{\sigma(1), \sigma(k)\}=\left\{w_{i}, w_{j}\right\}$. Considere o vértice $u_{r+1}$. Note que $u_{r+1}$ é adjacente a $w_{i}$ e a $w_{j}$, pois $1 \leq i, j \leq r$. Também note que $f \geq 2 \Rightarrow N\left(u_{r+1}\right) \subsetneq W$. Assim, $u_{r+1}$ não possui a propriedade da vizinhança convexa, uma vez que $u_{r+1}$ é adjacente a $w_{i}$ e $w_{j}$, isto é, adjacente a $\sigma(1)$ e $\sigma(k)$, mas possui estritamente menos que $k=|W|$ vizinhos, já que $N\left(u_{r+1}\right) \subsetneq W$.

Seja $c \in \mathbb{Q}$ tal que $0<c \leq 1$ e considere a família de grafos

$$
\mathcal{F}^{c}=\{C F C G(k-\lfloor c k\rfloor+1,\lfloor c k\rfloor-1) \mid k \in \mathbb{N},\lfloor c k\rfloor \geq 3\} .
$$

Cada grafo em $\mathcal{F}^{c}$ é um grafo Ferrers-coroa completo com $2 k$ vértices e com convexidade igual a $\lfloor c k\rfloor$, por conta do que mostra a Proposição 7.22. Conforme mencionamos, nenhum dos argumentos de simplificação é efetivo para um grafo Ferrers-coroa completo, como é o caso dos membros de $\mathcal{F}^{c}$. A comparação de cada uma das cotas expostas, aplicadas a $\mathcal{F}^{c}$, é feita no próximo teorema.

Teorema 7.23. Seja $c \in \mathbb{Q}$ tal que $0<c \leq 1$ e seja $\mathcal{F}^{c}$ a família de grafos Ferrers-coroa completos $\{C F C G(k-\lfloor c k\rfloor+1,\lfloor c k\rfloor-1) \mid k \in \mathbb{N},\lfloor c k\rfloor \geq 3\}$. Para os grafos desta família, os expoentes das cotas da classe minima, da arboricidade, dos graus máximos, do número de arestas e da convexidade podem ser limitados superior e inferiormente por funções lineares em k. A saber:

$$
\begin{array}{rlrlrl}
(1-c) k & \leq & |U|-\mathfrak{C} & & \leq(1-c) k+1 \\
k & \leq & \min \{|U|,|W|\} & \leq k \\
& \left(1-\frac{c^{2}}{2}\right) k-3 & \leq & 2 \Upsilon & & \leq k+2 \\
k & \leq & \Delta_{U}=\Delta_{W} & \leq k \\
\sqrt{(1-c)} k & \leq & \sqrt{|E|+1} & & \leq k+1 .
\end{array}
$$

Em particular, para a família $\mathcal{F}^{c}$, a cota da convexidade é, assintoticamente, a mais justa.

Prova. Seja $k \in \mathbb{N}$ com $\lfloor c k\rfloor \geq 3$, e seja $G=G(c, k)$ o grafo de índice $k$ da família $\mathcal{F}^{c}$. Sejam, ainda, $U$ e $W$ suas classes de vértices, $\Upsilon$ sua arboricidade e $E$ seu conjunto de arestas.

A afirmação sobre a cota da classe mínima é clara, uma vez que as classes de vértices de qualquer grafo Ferrers-coroa possuem a mesma cardinalidade $k$. Além disso, os vértices $u_{k}$ e $w_{k-\lfloor c k\rfloor+2}$ são adjacentes a todos os vértices da classe oposta, e portanto, o grau máximo dentre os vértices de $U$ (dentre os vértices de $W$ ) é $k$. 
Com relação à cota da convexidade, temos que seu expoente, $|U|-\mathfrak{C}(G)$, pela Proposição 7.22 , é igual a $k-\lfloor c k\rfloor$. Assim, claramente,

$$
k-c k \leq k-\lfloor c k\rfloor \leq k-c k+1 \Leftrightarrow(1-c) k \leq|U|-\mathfrak{C}(G) \leq(1-c) k+1 .
$$

O número de arestas de $G$ é

$$
|E|=\frac{(\lfloor c k\rfloor-1)}{2}(k-\lfloor c k\rfloor+2+k)+(k-\lfloor c k\rfloor+1)(k-1) .
$$

Em particular,

$$
\begin{aligned}
|E| & \geq(k-\lfloor c k\rfloor+1)(k-1) \\
& \geq(k-c k+1)(k-1)=(1-c) k^{2}+c k-1,
\end{aligned}
$$

e portanto, $\sqrt{|E|+1} \geq \sqrt{(1-c)} k$. A simples observação de que $|E| \leq k^{2}$ estabelece que $\sqrt{|E|+1} \leq \sqrt{k^{2}+1}<k+1$.

Com relação à arboricidade de $G$, utilizamos as equações (7.6) e $|V(G)|=2 k$, a fim de aplicar o Corolário $7.6 \mathrm{com} H=G$, obtendo assim a seguinte cota inferior para $\Upsilon$ :

$$
\begin{aligned}
\Upsilon & \geq\left[\frac{(\lfloor c k\rfloor-1)}{2}(k-\lfloor c k\rfloor+2+k)+(k-\lfloor c k\rfloor+1)(k-1)\right] \cdot \frac{1}{2 k-1} \\
& \geq\left[\frac{(c k-2)}{2}(2 k-c k+2)+(k-c k+1)(k-1)\right] \cdot \frac{1}{2 k-1} \\
& =\left[\frac{2 c k^{2}-c^{2} k^{2}+4 c k-4 k-4}{2}+k^{2}-c k^{2}+c k-1\right] \cdot \frac{1}{2 k-1} \\
& =\left[\left(1-\frac{c^{2}}{2}\right) k^{2}+(3 c-2) k-3\right] \cdot \frac{1}{2 k-1} \geq\left(\frac{1}{2}-\frac{c^{2}}{4}\right) k-\frac{3}{2} \quad(\text { pois } k \geq 3),
\end{aligned}
$$

o que implica que

$$
2 \Upsilon \geq\left(1-\frac{c^{2}}{2}\right) k-3 .
$$

Por outro lado, podemos limitar superiormente a arboricidade de $G$ da seguinte maneira. Considere os grafos $G$ e $C G(2 k)$ como sendo subgrafos de $K_{k, k}$. Então, claramente vale que $\Upsilon(G) \leq \Upsilon\left(K_{k, k}\right)$. Além disso, uma l-floresta-decomposição qualquer de $C G(2 k)$ induz uma $(l+1)$-floresta-decomposição de $K_{k, k}$, pois o conjunto de arestas de $K_{k, k}$ difere do conjunto de arestas de $C G(2 k)$ apenas por um emparelhamento, e este corresponde a um subgrafo acíclico. Consequentemente, vale que $\Upsilon\left(K_{k, k}\right) \leq \Upsilon(C G(2 k))+1$. O Lema 7.11 garante que

$$
\Upsilon(C G(2 k))=\left\lceil\frac{k(k-1)}{2 k-1}\right\rceil \leq\left\lceil\frac{k-1}{2}\right\rceil \leq \frac{k}{2} .
$$

Portanto, por transitividade, temos que

$$
2 \Upsilon(G) \leq k+2 .
$$

Para a família $\mathcal{F}^{c}$, a cota da convexidade é, assintoticamente, a mais justa. De fato, temos que $c>0$ e que o expoente da cota da convexidade é limitado superiormente por $(1-c) k+1$. 
Ademais, os expoentes das demais cotas são limitadas inferiormente por funções lineares em $k$ cujos coeficientes angulares são estritamente maiores que $(1-c)$, a saber, $1-c^{2} / 2$ para a cota da arboricidade, $\sqrt{1-c}$ para a cota do número de arestas e 1 para as cotas da classe mínima e dos graus máximos.

Na próxima seção, verificaremos a existência de grafos para os quais a cota da convexidade se mostra justa, ou quase.

\subsection{Limite inferior para a cota da convexidade}

Nesta seção, trataremos da construção de famílias de grafos cujo número de bicliques maximais seja próximo da cota da convexidade, $O\left(2^{|U|-\mathfrak{c}|W|^{2}}\right)$. A primeira família apresentada como cota inferior é essencialmente a família $C F C G(r, f)$ e, assim, será necessário que calculemos o número de bicliques maximais do grafo Ferrers(2k).

Proposição 7.24. O grafo Ferrers $(2 k)$ possui $k$ bicliques maximais.

Prova. Sejam $\left\{u_{1}, u_{2}, \ldots, u_{k}\right\}$ e $\left\{w_{1}, w_{2}, \ldots, w_{k}\right\}$ suas classes de vértices e $\pi_{2}(\mathfrak{B})$ o conjunto das segundas coordenadas de suas bicliques maximais. Por definição, para todo $i$, temos que $N\left(u_{i}\right)=\left\{w_{1}, w_{2}, \ldots, w_{i}\right\}$. Pela Proposição 5.1, temos que

$$
\pi_{2}(\mathfrak{B})=\left\{\bigcap_{u_{i} \in I} N\left(u_{i}\right) \mid I \subseteq U\right\} .
$$

Note que a interseção arbitrária de conjuntos da forma $\left\{w_{1}, w_{2}, \ldots, w_{i}\right\}$ é novamente um conjunto desta forma. Assim, o número de conjuntos em $\pi_{2}(\mathfrak{B})$, isto é, o número de bicliques maximais, é menor ou igual a $k$. As simples observações de que cada $N\left(u_{i}\right)$ pertence a $\pi_{2}(\mathfrak{B})$, e de que Ferrers $(2 k)$ possui a propriedade da vizinhança distinta em $U$ estabelecem que $\left|\pi_{2}(\mathfrak{B})\right| \geq k$, e portanto, $\left|\pi_{2}(\mathfrak{B})\right|=k$.

Considere um membro da família $C F C G(r, f)$, e sejam $F$ o grafo Ferrers $(2 f)$ e $C$ o grafo $C G(2 r)$, com classes de vértices $U_{F}, W_{F}$ e $U_{C}, W_{C}$, respectivamente. O grafo $C F C G(r, f)$ foi definido como a união disjunta de $F$ e $C$, mais a adição de arestas entre quaisquer vértices $u$ e $w$, onde $u$ pertence a $U_{F}$ e $w$ a $W_{C}$, ou $u$ pertence a $U_{C}$ e $w$ a $W_{F}$. Esta operação é chamada de soma direta dos grafos bipartidos $F$ e $C$, e o grafo resultante desta operação é denotado $F \oplus C$. Sobre o número de bicliques maximais de uma soma direta, podemos provar o seguinte:

Lema 7.25. Sejam $G_{1}$ e $G_{2}$ dois grafos bipartidos e seja $G=G_{1} \oplus G_{2}$. Então,

$$
|\mathfrak{B}(G)|=\left|\mathfrak{B}\left(G_{1}\right)\right| \cdot\left|\mathfrak{B}\left(G_{2}\right)\right| .
$$

Prova. Sejam $\left(A_{1}, B_{1}\right)$ e $\left(A_{2}, B_{2}\right)$ bicliques maximais de $G_{1}$ e $G_{2}$, respectivamente. Note que, no grafo $G$, cada $u \in A_{1}$ é adjacente a todo elemento de $B_{1} \cup W_{2}$. Analogamente, todo 
$u \in A_{2}$ é adjacente a todo elemento de $B_{2} \cup W_{1}$. Agora, vamos mostrar que $\left(A_{1} \cup A_{2}, B_{1} \cup B_{2}\right)$ é uma biclique maximal de $G$. De fato,

$$
\begin{aligned}
\bigcap_{u \in A_{1} \cup A_{2}} N(u) & =\bigcap_{u \in A_{1}} N(u) \cap \bigcap_{u \in A_{2}} N(u) \\
& =\left(B_{1} \cup W_{2}\right) \cap\left(B_{2} \cup W_{1}\right) \\
& =\left(B_{1} \cap B_{2}\right) \cup\left(B_{1} \cap W_{1}\right) \cup\left(W_{2} \cap B_{2}\right) \cup\left(W_{2} \cap W_{1}\right) \\
& =\emptyset \cup B_{1} \cup B_{2} \cup \emptyset=B_{1} \cup B_{2} .
\end{aligned}
$$

Um cálculo análogo mostra que $\bigcap_{w \in B_{1} \cup B_{2}} N(w)=A_{1} \cup A_{2}$, e assim, $\left(A_{1} \cup A_{2}, B_{1} \cup B_{2}\right)$ é uma biclique maximal de $G$.

Uma aplicação direta do Lema 7.25 sobre o grafo $C F C G(r, f)$, utilizando o fato de que este grafo é a soma direta entre $C G(2 r)$ e Ferrers $(2 f)$, resulta no seguinte corolário:

Corolário 7.26 .

$$
|\mathfrak{B}(C F C G(r, f))|=2^{r} \cdot f .
$$

O próximo teorema mostra uma família de grafos com três parâmetros, cujos membros possuem $\Omega\left(2^{|U|-\mathfrak{C}} \mathfrak{C}\right)$ bicliques maximais. Em uma subfamília de dois parâmetros, o número de bicliques maximais é $\Omega\left(2^{|U|-\mathfrak{C}}|W|\right)$, apresentando uma folga linear em $|W|$ perante a cota da convexidade.

Teorema 7.27. Para todo $k, l, c \in \mathbb{N}^{*}$ satisfazendo $l \geq k+1$ e $3 \leq c \leq k$, existe um grafo bipartido $G=(U, W, E) \operatorname{com}|U|=k,|W|=l, \mathfrak{C}(G)=c e$

$$
|\mathfrak{B}(G)|=2^{k-c+1} \cdot(c-1)+1 .
$$

Em particular, se $c=\alpha$ para uma constante racional $\alpha$ satisfazendo $0<\alpha<1$, então segue que $|\mathfrak{B}(G)|=2^{k-c+1}(\alpha l-1)+1=\Omega\left(2^{k-c} \cdot l\right)$.

Prova. Seja $f=c-1$ e $r=k-c+1$. Observe que $f \geq 2$. Tomamos $G_{1}$ como sendo o grafo $C F C G(r, f)$. Pela Proposição 7.22, a convexidade de $G_{1}$ é $f+1=c$, e pelo Corolário 7.26, o número de bicliques maximais de $G_{1}$ é $2^{r} \cdot f=2^{k-c+1}(c-1)$. Note que $G_{1}$ possui $r+f=k$ vértices em cada uma de suas classes.

Seja $G$ o grafo obtido após a adição de $l-k \geq 1$ vértices isolados à classe $W$ de $G_{1}$. Assim, $G$ possui $k$ vértices em $U$ e $l$ vértices em $W$. Claramente as convexidades de $G_{1}$ e $G$ são iguais, e portanto, $\mathfrak{C}(G)=c$. Também são iguais os números de bicliques maximais com arestas de $G_{1}$ e $G$. No entanto, $G$ possui exatamente uma biclique maximal sem arestas: $(\emptyset, W)$, ao passo que $G_{1}$ não possui nenhuma. Portanto, o resultado desta operação de adição de vértices é um grafo $G$ que atende as exigências do enunciado.

A partir de agora e até o fim desta seção, o objetivo buscado é mostrar uma família de grafos para a qual a cota da convexidade é assintoticamente justa. A fim de chegarmos a este resultado, será necessário estabelecermos primeiramente que a cota superior para grafos convexos, contida na Proposição 7.16, é assintoticamente justa.

Definiremos, assim, uma família de grafos convexos, que mostraremos possuir um número de bicliques maximais quadrático em $|W|$. Sejam $k, l \in \mathbb{N} \operatorname{com} k \leq\left\lceil\frac{l}{2}\right\rceil$. O grafo interordinal 
de $(k, l)$ é denotado $I G(k, l)$ e definido da seguinte maneira: $I G(k, l)=(U, W, E)$ onde $U=\left\{u_{1}, u_{2}, \ldots, u_{k}\right\}$ e $W=\left\{w_{1}, w_{2}, \ldots, w_{l}\right\}$ são dois conjuntos disjuntos e seu conjunto de arestas é definido por $E=\left\{u_{i} w_{j} \mid i=1, \ldots, k, j=i, \ldots, i+k-1\right\}$. Estamos particularmente interessados em casos em que $l=\left\lceil\frac{k}{2}\right\rceil$ e, por isso, retratamos na Figura 7.11 os grafos interordinais para $k$ variando de 1 até 6 , enquanto que $l=\left\lceil\frac{k}{2}\right\rceil$.

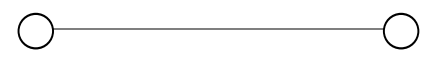

(a) $\operatorname{IG}(1,1)$

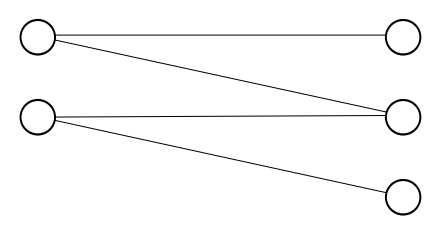

(d) $\operatorname{IG}(2,4)$

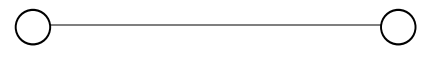

$\bigcirc$

(b) $\mathrm{IG}(1,2)$

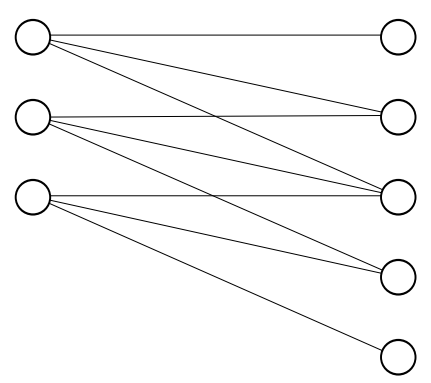

(e) $\operatorname{IG}(3,5)$

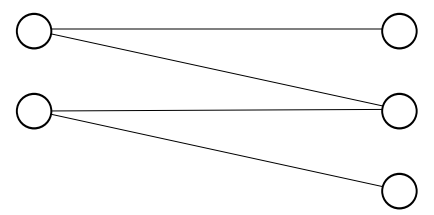

(c) $\operatorname{IG}(2,3)$

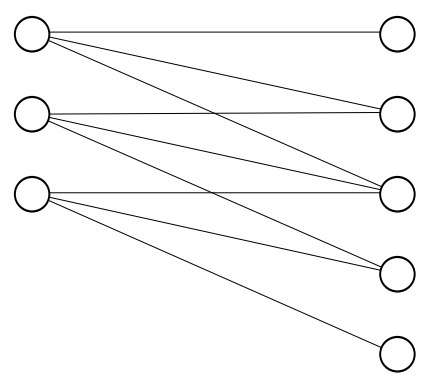

(f) $\operatorname{IG}(3,6)$

Figura 7.11: Grafos interordinais de ordem 1 até $6, \operatorname{com} l=\left\lceil\frac{k}{2}\right\rceil$.

O próximo lema apresenta o cálculo do número de bicliques maximais com arestas do grafo interordinal. Como consequência, seguirá que a cota superior sobre grafos convexos, presente na Proposição 7.16, é assintoticamente justa. De fato, por construção, o grafo $I G(k, l)$ é convexo sobre $W$ e possui $l$ vértices naquela classe e, de acordo com o Lema 7.28, no caso em que $k=\lceil l / 2\rceil$, temos que o número de bicliques maximais de $I G(k, l)=I G(\lceil l / 2\rceil, l)$ é pelo menos $\frac{1}{2}\left[\frac{l}{2}\left(\frac{l}{2}+1\right)\right]=\Omega\left(l^{2}\right)$.

Lema 7.28. Sejam $k, l \in \mathbb{N}^{*}$ com $k \leq\left\lceil\frac{l}{2}\right\rceil$. O número de bicliques maximais com arestas do grafo $I G(k, l)$ é

$$
\frac{k(k+1)}{2} .
$$

Prova. Sejam $U=\left\{u_{1}, u_{2}, \ldots, u_{k}\right\}$ e $W=\left\{w_{1}, w_{2}, \ldots, w_{l}\right\}$ as classes de vértices em questão.

Vamos mostrar que toda primeira coordenada de biclique maximal com arestas é um conjunto da forma

$$
\left\{u_{i}, u_{i+1}, \ldots, u_{i+p}\right\} \text {, para algum } i \in[k] \text { e algum } 0 \leq p \leq k-i .
$$

De fato, seja $A \neq \emptyset$ uma primeira coordenada de uma biclique maximal com arestas. Se $A$ contiver apenas um elemento, não há o que provar. Caso contrário, sejam $u_{i}, u_{j}$ elementos 
de $A$ com $i<j$. Pela construção de $I G(k, l)$, temos que $N\left(u_{i}\right)=\left\{w_{i}, w_{i+1}, \ldots, w_{i+k-1}\right\}$ e que $N\left(u_{j}\right)=\left\{w_{j}, w_{j+1}, \ldots, w_{j+k-1}\right\}$. Claramente:

$$
j \leq k \text { e } i \geq 1 \Rightarrow j-i<k \Rightarrow j \leq i+k-1
$$

assim como $i<j \Rightarrow i+k-1 \leq j+k-1$. Logo,

$$
N\left(u_{i}\right) \cap N\left(u_{j}\right)=\left\{w_{j}, w_{j+1} \ldots, w_{i+k-1}\right\} .
$$

Seja $B=\cap_{u_{x} \in A} N\left(u_{x}\right)$ a segunda coordenada da biclique maximal associada a $A$. Do fato de $u_{i}, u_{j} \in A$ e de $(A, B)$ ser biclique, temos claramente que

$$
B \subseteq N\left(u_{i}\right) \cap N\left(u_{j}\right)
$$

Agora mostraremos que, para todo $x$ tal que $i \leq x \leq j$, vale que $u_{x} \in A$. De $j \geq x$ e $i \leq x$, concluímos que $N\left(u_{x}\right)=\left\{w_{x}, w_{x+1}, \ldots, w_{x+k-1}\right\} \supseteq\left\{w_{j}, \ldots, w_{i+k-1}\right\}$. Assim, temos que vale que $N\left(u_{x}\right) \supseteq N\left(u_{i}\right) \cap N\left(u_{j}\right)$, e portanto, $N\left(u_{x}\right) \supseteq B$. Então, para todo $w \in B$, existe a aresta $u_{x} w$. Como $(A, B)$ é maximal, concluímos que $u_{x} \in A$. Consequentemente, $A$ é um conjunto da forma mencionada.

Agora mostraremos a recíproca, isto é, será provado que todo conjunto da forma

$$
\left\{u_{i}, u_{i+1}, \ldots, u_{i+p}\right\} \text {, para algum } i \in[k] \text { e algum } 0 \leq p \leq k-i,
$$

é a primeira coordenada de alguma biclique maximal com arestas. Seja $S=\left\{u_{i}, u_{i+1}, \ldots, u_{i+p}\right\}$ um tal conjunto, e seja $B=\bigcap_{j=0}^{p} N\left(u_{i+j}\right)=\left\{w_{i+p}, w_{i+p+1}, \ldots, w_{i+k-1}\right\}$. Claramente, $(S, B)$ é uma biclique. Vamos mostrar que $(S, B)$ é maximal e possui arestas.

Primeiramente, mostramos que não existe $T \supsetneq S$ tal que $(T, B)$ é uma biclique (isto é, o par $(S, B)$ é uma biclique $U$-maximal). Seja $u_{x} \in U \backslash S$ e, portanto, $x<i$ ou $x>i+p$. Suponha o caso em que $x<i$. Então, temos que $x+k-1<i+k-1$, e portanto, $u_{x}$ não é adjacente a $w_{i+k-1}$. Agora, suponha o caso em que $x>i+p$. Então, temos que $u_{x}$ não é adjacente a $w_{i+p}$. Em qualquer um dos dois casos, temos que $u_{x}$ não é adjacente algum vértice de $B$, e portanto $(S, B)$ é $U$-maximal.

Por outro lado, a escolha de $B$, como intersecção de adjacências, garante que $(S, B)$ também é $W$-maximal, e portanto, trata-se de uma biclique maximal.

Resta mostrarmos que $(S, B)$ possui arestas. Claramente, temos que $S \neq \emptyset$. Além disso, o vértice $w_{k}$ pode ser facilmente verificado como adjacente a todo $u \in U$ e, portanto, a todo $u \in S$, e, assim, pela $W$-maximalidade de $(S, B)$, segue que $w_{k} \in B \Rightarrow B \neq \emptyset$.

O número de conjuntos da forma $\left\{u_{i}, u_{i+1}, \ldots, u_{i+p}\right\}$ com $i \in[k]$ e $0 \leq p \leq k-i$ é

$$
k+(k-1)+\ldots+1=\frac{k(k+1)}{2} .
$$

A fim de estender o resultado do lema anterior para a cota da convexidade, consideraremos a seguinte construção. A união disjunta de um grafo interordinal, $I G=I G\left(\left\lceil l_{1} / 2\right\rceil, l_{1}\right)$, e um grafo coroa de tamanho "constante", resulta em um grafo de convexidade elevada (no sentido da diferença entre $|U|$ e $\mathfrak{C}$ ser constante) e com pelo menos $|\mathfrak{B}(I G)|=\Theta\left(l_{1}^{2}\right)$ 
bicliques maximais. Sejam $k$ e $l$ o número de vértices das classes $U$ e $W$ deste grafo. Note que $l_{1}=\Theta(l)$. Pelo fato da convexidade ser elevada, temos que a cota da convexidade, neste caso, é o produto de uma função quadrática em $l$ por $2^{\alpha}$, onde $\alpha=k-\mathfrak{C}$ é uma constante. Neste caso, o número de bicliques maximais, assim como a cota da convexidade, pertencem a $\Theta\left(l^{2}\right)$. O conteúdo do próximo teorema é um desenvolvimento mais formal desta argumentação. Como resultado, segue a construção de uma família infinita, de um parâmetro, para a qual a cota da convexidade é assintoticamente justa.

Teorema 7.29. Para todo $k, l, c \in \mathbb{N}^{*}$ satisfazendo $l \geq k+2$ e $3 \leq c \leq k$, existe um grafo bipartido $G=(U, W, E)$ com $|U|=k,|W|=l, \mathfrak{C}(G)=c e$

$$
|\mathfrak{B}(G)|=2^{k-c+2}+\frac{1}{2}\left(\left\lceil\frac{c}{2}\right\rceil-2\right)\left(\left\lceil\frac{c}{2}\right\rceil-1\right)+2 .
$$

Em particular, se $c=k-\alpha$ e $k=\beta l$ para duas constantes $\alpha, \beta$ satisfazendo $0 \leq \alpha \leq k$ e $0<\beta<1$, então vale que $|\mathfrak{B}(G)|=2^{\alpha+2}+\frac{1}{2}\left(\left\lceil\frac{\beta l-\alpha}{2}\right\rceil-2\right)\left(\left\lceil\frac{\beta l-\alpha}{2}\right\rceil-1\right)+2=\Omega\left(l^{2}\right)$. Neste caso, a cota da convexidade é assintoticamente justa.

Prova. Seja $G_{C}$ o grafo coroa $C G(2(k-c+2))$, e sejam assim $U_{C}=\left\{u_{1}, u_{2}, \ldots, u_{k-c+2}\right\}$ e $W_{C}=\left\{w_{1}, w_{2}, \ldots, w_{k-c+2}\right\}$ suas classes de vértices, de maneira que $E\left(G_{c}\right)=\left\{u_{i} w_{j} \mid i \neq j\right\}$. Seja $G_{I G}$ o grafo $I G\left(\left\lceil\frac{c}{2}\right\rceil-2, c\right)$, com classes de vértices $U_{I G}=\left\{u_{k-c+3}, u_{k-c+4}, \ldots, u_{k-c+\lceil c / 2\rceil}\right\}$ e $W_{I G}=\left\{w_{k-c+3}, w_{k-c+4}, \ldots, w_{k+2}\right\}$. Seja, ainda, $G_{1}$ a cópia disjunta dos grafos $G_{C}$ e $G_{I G}$, e sejam $U_{1}=U_{C} \cup U_{I G}$ e $W_{1}=W_{C} \cup W_{I G}$ as classes de $G_{1}$. Assim:

$$
\begin{aligned}
U_{1} & =\left\{u_{1}, u_{2}, \ldots, u_{k-c+\lceil c / 2\rceil}\right\} \\
W_{1} & =\left\{w_{1}, w_{2}, \ldots, w_{k+2}\right\} .
\end{aligned}
$$

Sendo $G_{1}$ dado pela união disjunta dos grafos $G_{C}$ e $G_{I G}$, temos que, pela Proposição 7.15, o número de bicliques maximais com arestas de $G_{1}$ é a soma das bicliques maximais com arestas de $G_{C}$ e $G_{I G}$, isto é, $2^{k-c+2}+\frac{1}{2}\left(\left\lceil\frac{c}{2}\right\rceil-2\right)\left(\left\lceil\frac{c}{2}\right\rceil-1\right)$. É imediato verificar que $G_{1}$ possui as duas bicliques maximais sem arestas possíveis, e, portanto,

$$
\left|\mathfrak{B}\left(G_{1}\right)\right|=2^{k-c+2}+\frac{1}{2}\left(\left\lceil\frac{c}{2}\right\rceil-2\right)\left(\left\lceil\frac{c}{2}\right\rceil-1\right)+2 .
$$

Tomamos $G=(U, W, E)$ como sendo $G_{1}$ após a adição de $c-\left\lceil\frac{c}{2}\right\rceil$ vértices isolados a $U_{1}$ e $l-k-2$ vértices isolados a $W_{1}$. Assim, $G$ possui, nas classes $U$ e $W$, respectivamente, $k$ e $l$ vértices. Verifica-se facilmente que cada biclique maximal com arestas de $G$ também é uma biclique maximal com arestas de $G_{1}$, e reciprocamente. Além disso, $G$ também possui duas bicliques maximais sem arestas. Logo, temos que $|\mathfrak{B}(G)|=\left|\mathfrak{B}\left(G_{1}\right)\right|$. Assim, resta somente mostrar que a convexidade de $G$ é $c$.

Seja $\sigma:[l] \rightarrow W$ dada por $\sigma(i)=w_{i}$. Pela construção do grafo interordinal, cada vértice de $U_{I G}$ possui vizinhança convexa sob $\sigma$, assim como os vértices $u_{1}$ e $u_{k-c+2}$ de $U_{C}$, bem como os $c-\left\lceil\frac{c}{2}\right\rceil$ vértices isolados adicionados a $U$, perfazendo um total de $c$ vértices. Isto mostra que $\mathfrak{C}(G) \geq c$.

Vamos mostrar agora que no máximo dois vértices de $U_{C}$ possuem vizinhança convexa sob qualquer bijeção $\tau:[l] \rightarrow W$. Note que isto implica que $\mathfrak{C}(G) \leq c$, pois $\left|U_{C}\right|=k-c+2$ e $|U|=k \Rightarrow\left|U \backslash U_{C}\right|=c-2$. Definimos $r=k-c+2=\left|U_{C}\right|$. Seja $\tau:[l] \rightarrow W$ uma 
bijeção, e sejam $u_{i}, u_{j} \in U_{C}$ com $i \neq j$ dois vértices com vizinhança convexa sob $\tau$. Note que $N\left(u_{i}\right) \cap N\left(u_{j}\right)=r-2$. Como $N\left(u_{i}\right)$ e $N\left(u_{j}\right)$ são $\tau$-intervalos, segue que $N\left(u_{i}\right) \cap N\left(u_{j}\right)$ também é. Além disso, como $N\left(u_{i}\right) \neq N\left(u_{j}\right)$ e $\left|N\left(u_{i}\right)\right|=\left|N\left(u_{j}\right)\right|$, temos que o vértice $\tau(1)$ não pertence a ambos os conjuntos, bem como o vértice $\tau(l)$. Consequentemente, temos que existe $x \in\{2,3, \ldots, l-r+2\}$ com

$$
N\left(u_{i}\right) \cap N\left(u_{j}\right)=\{\tau(x), \tau(x+1), \ldots, \tau(x+r-3)\} .
$$

Note que $N\left(u_{i}\right) \backslash N\left(u_{j}\right)=\left\{w_{j}\right\}$ e que $N\left(u_{j}\right) \backslash N\left(u_{i}\right)=\left\{w_{i}\right\}$, pela definição de adjacências do grafo $C G(2 k)$. Do fato de $N\left(u_{i}\right)$ e $N\left(u_{j}\right)$ serem $\tau$-intervalos distintos, e também de (7.7), concluímos que $\tau(x-1)=w_{i}$ e $\tau(x+r-2)=w_{j}$ ou $\tau(x-1)=w_{i}$ e $\tau(x+r-2)=w_{j}$. Assim, todo $\tau$-intervalo que contenha $w_{i}$ e $w_{j}$ possui no mínimo $x+r-2-(x-1)+1=r$ elementos. Por fim, seja $u_{s} \in U_{C} \operatorname{com} s \neq i, s \neq j$. De $s \neq i$ e $s \neq j$, temos que $u_{s}$ é adjacente a $w_{i}$ e $w_{j}$. Como o grau de $u_{s}$ é $r-1$, temos que $N\left(u_{s}\right)$ não pode ser um $\tau$-intervalo, em virtude de $\left|N\left(u_{s}\right)\right|=r-1$ e de $w_{i}, w_{j} \in N\left(u_{s}\right)$. Em outras palavras, $u_{s}$ não possui a propriedade da vizinhança convexa.

No caso em que $c=k-\alpha$ para uma constante $0 \leq \alpha \leq k$, temos que a cota da convexidade é $2^{|U|-\mathfrak{C}} \cdot[|W|(|W|+1) / 2+1]=2^{\alpha} \cdot[l(l+1) / 2+1]=O\left(l^{2}\right)$. Se, adicionalmente, tivermos que $k=\beta l$ para uma constante $0<\beta<1$, então temos que o número de bicliques maximais do grafo $G$ é

$$
|\mathfrak{B}(G)|=\left|\mathfrak{B}\left(G_{1}\right)\right|=2^{\alpha+2}+\frac{1}{2}\left(\left\lceil\frac{\beta l-\alpha}{2}\right\rceil-2\right)\left(\left\lceil\frac{\beta l-\alpha}{2}\right\rceil-1\right)=\Omega\left(l^{2}\right) .
$$

Na próxima seção, abordaremos a questão do cálculo da convexidade de um grafo bipartido.

\subsection{Complexidade do cálculo de convexidade}

A convexidade sobre $W$ de um grafo bipartido foi definida em (7.5) como um máximo tomado sobre todas as bijeções $\sigma:[|W|] \rightarrow W$. Desta maneira, uma computação de $\mathfrak{C}(G)$ diretamente através de sua definição mostra-se rapidamente impraticável, uma vez que o número de tais bijeções cresce conforme o fatorial de $|W|$. Provaremos nesta seção que a existência de um algoritmo polinomial para o cálculo da convexidade implica que $\mathbf{P}=\mathbf{N P}$. Mais especificamente, consideraremos o seguinte problema de decisão:

\section{Problema CONV}

Entrada: Um grafo bipartido $G=(U, W, E)$ e um número natural $c$.

Saída: SIM, se $\mathfrak{C}(G) \geq c ;$ NÃO, caso contrário.

Provaremos no Teorema 7.30 que CONV é NP-completo. A redução de tempo polinomial será realizada a partir de um problema sobre um grafo completo, munido de uma função de distância $d: E \rightarrow\{1,2\}$ sobre suas arestas. Neste cenário, definimos que uma aresta $e \in E$ é curta se $d(e)=1$, e longa se $d(e)=2$. O problema mencionado é: 


\section{Problema TSP- $\{1,2\}$-SEM-RETORNO}

Entrada: Um grafo completo $K_{n}$, uma função de distância $d: E\left(K_{n}\right) \rightarrow\{1,2\}$ e um número natural $b$.

Saída: SIM, se existir um caminho hamiltoniano em $K_{n}$ com pelo menos $b$ arestas curtas; NÃO, caso contrário.

Note que, em uma instância do problema TSP-\{1,2\}-SEM-RETORNO, vale que existe um caminho hamiltoniano com pelo menos $b$ arestas curtas se e só se existe um caminho hamiltoniano de distância total percorrida menor ou igual a $2(n-1)-b$. Assim, o problema TSP-\{1,2\}-SEM-RETORNO é NP-completo, conforme [GJ79, p. 211].

Para simplificarmos a descrição, supomos que o conjunto de vértices de um grafo completo proveniente de uma instância do problema TSP-\{1,2\}-SEM-RETORNO é sempre o conjunto de naturais $\{1,2, \ldots, n\}$. Chamaremos os elementos deste conjunto de cidades.

Teorema 7.30. O problema CONV é NP-completo. A pertinência deste problema à classe dos problemas NP-completos se mantém quando suas instâncias são restringidas a grafos bipartidos cujos vértices de $U$ possuem grau igual a dois.

Prova. Claramente o problema pertence à classe NP, uma vez que, dados $G=(U, W, E)$, uma bijeção $\sigma$ de $\{1,2, \ldots,|W|\}$ em $W$ e um natural $c$, o teste $\operatorname{conv}(G, \sigma) \geq c$ pode ser conduzido em tempo polinomial.

Seja $\mathcal{I}=\left(K_{n}, d\right)$ uma instância do TSP- $\{1,2\}$-SEM-RETORNo. Definimos um grafo bipartido $G_{\mathcal{I}}=\left(U_{\mathcal{I}}, W_{\mathcal{I}}, E_{\mathcal{I}}\right)$, onde:

- Para cada cidade $i \in[n]$, existe um vértice $w_{i} \in W_{\mathcal{I}}$ biunivocamente associado.

- Para cada aresta curta $i j \in E\left(K_{n}\right)$, existe um vértice $u_{i j} \in U_{\mathcal{I}}$ e duas arestas: $u_{i j} w_{i}, u_{i j} w_{j} \in E_{\mathcal{I}}$.

Observe que a construção de $G_{\mathcal{I}}$, a partir da instância $\mathcal{I}$, pode ser realizada em tempo polinomial em $n$. Além disso, todo vértice $u \in U_{\mathcal{I}}$ possui grau igual a dois.

Para cada bijeção $\sigma:[n] \rightarrow W_{\mathcal{I}}$, associaremos um caminho hamiltoniano no grafo $K_{n}$, que será denotado $P(\sigma)$ e dado da seguinte maneira. A sequência de cidades visitadas por $P(\sigma)$ são os índices dos elementos da sequência $(\sigma(1), \sigma(2), \ldots, \sigma(n))$, nesta mesma ordem. Note que todo caminho hamiltoniano do $K_{n}$ pode ser obtido desta forma, isto é, para todo caminho hamiltoniano $Q$ do grafo $K_{n}$, existe uma bijeção $\sigma:[n] \rightarrow W_{\mathcal{I}}$ tal que $P(\sigma)=Q$.

Seja $\sigma:[n] \rightarrow W_{\mathcal{I}}$ uma bijeção. Vamos mostrar que $\operatorname{conv}\left(G_{\mathcal{I}}, \sigma\right)$ é igual ao número de arestas curtas de $P(\sigma)$. Para isso, mostraremos primeiramente que os vértices de $U$ com vizinhança convexa sob $\sigma$ correspondem injetivamente às arestas curtas de $P(\sigma)$.

Seja $u_{i j} \in U_{\mathcal{I}}$ um vértice com a propriedade da vizinhança convexa sob $\sigma$. Então, temos que $N\left(u_{i j}\right)=\{\sigma(k), \sigma(k+1)\}$, para algum $k \in\{1,2, \ldots, n-1\}$. Note que, por construção, se $u_{i j} \in U_{\mathcal{I}}$, então $i j$ é uma aresta curta de $K_{n}$. Novamente por construção, vale que $N\left(u_{i j}\right)=\left\{w_{i}, w_{j}\right\}$, e portanto $\{\sigma(k), \sigma(k+1)\}=\left\{w_{i}, w_{j}\right\}$. Consequentemente, a $k$-ésima e a $(k+1)$-ésima cidades visitadas por $P(\sigma)$ são $i$ e $j$ ou $j$ e $i$. Em qualquer um dos casos, 
a aresta curta $i j$ é percorrida pelo caminho $P(\sigma)$. Esta correspondência entre $u_{i j}$ e $i j$ é claramente injetiva.

Agora, mostraremos uma correspondência injetiva no sentido contrário. Suponha que $e=i j$ seja uma aresta curta percorrida pelo caminho hamiltoniano $P(\sigma)$. Assim, existe $k \in\{1,2, \ldots, n-1\}$ tal que $\{\sigma(k), \sigma(k+1)\}=\left\{w_{i}, w_{j}\right\}$. Como $i j$ é aresta curta, então, por construção, existe um vértice $u_{i j} \in U_{\mathcal{I}}$ cujo conjunto de vizinhos é $\left\{w_{i}, w_{j}\right\}=\{\sigma(k), \sigma(k+1)\}$. Logo, $u_{i j}$ possui vizinhança convexa sob $\sigma$. Esta associação também é claramente injetiva.

Provamos assim que conv $\left(G_{\mathcal{I}}, \sigma\right)$ é igual ao número de arestas curtas em $P(\sigma)$. Como para todo caminho hamiltoniano $Q$, existe $\sigma$ tal que $P(\sigma)=Q$, temos que existe um caminho hamiltoniano com pelo menos $b$ arestas curtas no grafo $K_{n}$ se e somente se existe bijeção $\sigma$ tal que $\operatorname{conv}\left(G_{\mathcal{I}}, \sigma\right) \geq b$, isto é, se e somente se $\mathfrak{C}\left(G_{\mathcal{I}}\right) \geq b$.

Um aspecto interessante do cálculo de convexidade é que, foi mostrado por Booth e Lueker um algoritmo de tempo $O(\max \{|U|,|W|\}+|E|)$ para o reconhecimento de um grafo convexo [BL75]. Isto é, apesar do cálculo de $\mathfrak{C}(G)$ ser NP-difícil, como mostramos, decidir se $\mathfrak{C}(G)=|U|$ é um problema solúvel em tempo polinomial. 


\section{Capítulo 8}

\section{Conclusão e trabalhos futuros}

Realizamos nesta dissertação uma resenha da Análise de Conceitos Formais. Sua fundamentação foi apresentada da maneira tradicional, algébrica, bem como sob a linguagem da teoria dos grafos. O aspecto algorítmico foi abordado, com a exposição de métodos para a determinação do reticulado de conceitos, e de uma subestrutura simplificada deste. Expomos aplicações, munidas de dados reais, na área de análise de dados exploratória e recuperação de informação.

Dada a existência de famílias de grafos bipartidos com um número exponencial de bicliques maximais, um problema combinatório importante que se coloca é o estabelecimento de cotas superiores para o número de bicliques maximais de um grafo bipartido. Neste trabalho, expomos todas as cotas superiores encontradas por nós na literatura e estabelecemos um limite inferior mais justo para uma delas. Definimos uma nova invariante em grafos bipartidos, e baseados nela provamos uma nova cota superior, e construímos famílias de casos para os quais nosso resultado provê uma limitação mais justa que as demais cotas. Em particular, mostramos famílias infinitas para as quais nossa cota é limitada por uma função polinomial, enquanto que as demais cotas crescem exponencialmente. Limites inferiores para nossa cota foram mostrados. Também estabelecemos a complexidade computacional do cálculo da nova invariante, a convexidade de um grafo bipartido.

Nossa cota superior foi apresentada em conferência [AdL11], e uma compilação abrangente dos resultados originais foi submetida a revista e aguarda resposta.

Uma questão em aberto que se coloca naturalmente é: existem algoritmos de aproximação para o cálculo da convexidade? Esta questão se mostra particularmente relevante

se considerarmos que o teorema da cota da convexidade permance válido quando trocamos a convexidade por uma aproximação inferior qualquer.

\section{Trabalhos futuros}

Além da questão citada, delineamos em um horizonte futuro as seguintes frentes de pesquisa.

Convexidade Apesar do cálculo de convexidade ser NP-difícil, temos resultados preliminares que indicam que o seu cálculo em algumas classes é um problema solúvel em tempo poli- 
nomial. Uma direção a seguir é investigar a respeito destas classes, e discutir sua abundância nas aplicações de FCA.

Uma outra frente de atuação seria relacionar convexidade com propriedades e invariantes de grafos, e conseguir estabelecer cotas inferiores para convexidade, de maneira a ainda assim ser capaz de utilizar uma cota superior, com um resultado eventualmente aproximado.

Imersões Como mostramos, reticulados de conceitos podem transmitir informação de maneira valiosa a um usuário. Essa poderosa capacidade requer uma representação geométrica apropriada, e assim, os métodos da área de graph drawing são fundamentais para as aplicações de FCA. Nesta área, se inserem diversos problemas combinatórios como minimização de cruzamento de arestas e maximização de simetrias, e pesquisa específica para desenhos de reticulados vem sendo desenvolvida, como em [EV10], [Zsc07], [Fre04] e [Col01].

Aplicações não interativas As aplicações exibidas neste trabalho são fundamentalmente interativas: todo reticulado é apresentado a um usuário que realiza análise. Existem resultados na literatura sobre aplicações não interativas, como por exemplo [HFC07]. Esse tipo de aplicação promove ainda mais a pesquisa sobre algoritmos eficientes de construção de reticulados de conceitos, como os explorados no Capítulo 6. 


\section{Referências Bibliográficas}

$\left[\mathrm{AAC}^{+} 04\right]$ Gabriela Alexe, Sorin Alexe, Yves Crama, Stephan Foldes, Peter L. Hammer, and Bruno Simeone. Consensus algorithms for the generation of all maximal bicliques. Discrete Applied Mathematics, 145(1):11-21, 2004. 118

[AdL11] Alexandre Albano and Alair Pereira do Lago. New upper bound for the number of maximal bicliques of a bipartite graph. In 10th Cologne-Twente Workshop on Graphs and Combinatorial Optimization, Frascati, Italy, 2011. 137

[BC04] Peter Becker and Joachim Hereth Correia. The ToscanaJ suite for implementing Conceptual Information Systems. In Formal Concept Analysis: State of the Art, Berlin Heidelberg. Springer, 2004. 16, 51, 61

[BL75] Kellogg S. Booth and George S. Lueker. Linear algorithms to recognize interval graphs and test for the consecutive ones property. In Proc. of 7th annual ACM symposium on Theory of computing, STOC '75, pages 255-265, NY, USA, 1975. ACM. 136

[Bor86] Jean-Paul Bordat. Calcul pratique du treillis de Galois d'une correspondance. Mathématiques et Sciences Humaines, 96:31-47, 1986. 17

[Col01] Richard Cole. Automated layout of concept lattices using layered diagrams and additive diagrams. In $A C S C$, pages 47-53. IEEE Computer Society, 2001. 138

[CR04] Claudio Carpineto and Giovanni Romano. Concept Data Analysis: Theory and Applications. John Wiley \& Sons, 2004. 15, 17, 63

[CSWW03] Joachim Hereth Correia, Gerd Stumme, Rudolf Wille, and Uta Wille. Conceptual knowledge discovery - a human-centered approach. Applied Artificial Intelligence, 17(3):281-302, 2003. 62

[DH98] Stephan Düwel and Wolfgang Hesse. Identifying candidate objects during system analysis. In Third CAISE'98/IFIP 8.1 International Workshop on Evaluation of Modeling Methods in System Analysis and Design, Pisa, 1998. 62

[Die10] Reinhard Diestel. Graph Theory, volume 173 of Graduate Texts in Mathematics. Springer, Heidelberg, 4th edition, 2010. 108

[DP02] B. A. Davey and H. A. Priestley. Introduction to Lattices and Order. Cambridge University Press, 2002. 35, 36 
[Epp94] David Eppstein. Arboricity and bipartite subgraph listing algorithms. Information Processing Letters, 51(4):207-211, 1994. 17, 108, 109, 112

[EV10] Peter W. Eklund and Jean Villerd. A survey of hybrid representations of concept lattices in conceptual knowledge processing. In Léonard Kwuida and Baris Sertkaya, editors, ICFCA, volume 5986 of Lecture Notes in Computer Science, pages 296-311. Springer, 2010. 138

[Fre04] Ralph Freese. Automated lattice drawing. In Concept Lattices. Proc. 2nd International Conf. on Formal Concept Analysis (ICFCA 04), pages 112-127. Springer, 2004. 138

[Gan84] Bernhard Ganter. Two basic algorithms in concept analysis. FB4-Preprint 831, TH Darmstadt, 1984. 17, 74

[Gan10] Bernhard Ganter. Personal communication, 2010. 16, 26

[GJ79] Michael R. Garey and David S. Johnson. Computers and Intractability, A Guide to the Theory of NP-Completeness. W.H. Freeman and Company, New York, 1979. 45, 135

[Gro96] Anja Großkopf. Formal concept analysis of verb paradigms in linguistics. Ordinal and symbolic data analysis, pages 70-79, 1996. 62

[GW99] Bernhard Ganter and Rudolf Wille. Formal Concept Analysis: Mathematical Foundations. Springer, Berlin-Heidelberg, 1999. 115

[HFC07] Yang Huang and Martin Farach-Colton. Lattice based clustering of temporal gene-expression matrices. In SDM. SIAM, 2007. 138

[HSWW00] Joachim Hereth, Gerd Stumme, Rudolf Wille, and Uta Wille. Conceptual knowledge discovery and data analysis. In Proceedings of the Linguistic on Conceptual Structures: Logical Linguistic, and Computational Issues, pages 421-437, London, UK, 2000. Springer-Verlag. 62

[KKV00] Beate Kohler-Koch and Frank Vogt. Normen und regelgeleitete internationale Kooperationen, pages 325-340. Springer, Berlin - Heilderberg - New York, 2000. 62

[Kro67] M. R. Krom. The decision problem for a class of first-order formulas in which all disjunctions are binary. Zeitschrift für mathematische Logik und Grundlagen der Mathematik, 13:15-20, 1967. 46

[KSVW94] W. Kollewe, M. Skorsky, F. Vogt, and R. Wille. TOSCANA - ein Werkzeug zur begrifflichen Analyse und Erkundung von Daten. In R. Wille and M. Zickwolff, editors, Begriffliche Wissensverarbeitung-Grundfragen und Aufgaben, pages 267288, Mannheim, 1994. B. I. -Wissenschaftsverlag. 61 
[Kuz01] Sergei O. Kuznetsov. On computing the size of a lattice and related decision problems. Order, 18(4):313-321, 2001. 16, 47, 115

[LS97] Christian Lindig and Gregor Snelting. Assessing modular structure of legacy code based on mathematical concept analysis. In Proceedings of the 19th international conference on Software engineering, ICSE '97, pages 349-359, New York, NY, USA, 1997. ACM. 62

[MRS08] Christopher D. Manning, Prabhakar Raghavan, and Hinrich Schütze. Introduction to Information Retrieval. Cambridge University Press, July 2008. 62

[NR99] Lhouari Nourine and Olivier Raynaud. A fast algorithm for building lattices. Information Processing Letters, 71(5-6):199-204, 1999. 17, 87

[NW61] C. St J. A. Nash-Williams. Edge-disjoint spanning trees of finite graphs. Journal of London Mathemathical Society, 36, 1961. 108

[NW64] C. St.J. A. Nash-Williams. Decomposition of finite graphs into forests. Journal of the London Mathematical Society, s1-39(1):12, 1964. 17, 108

[PDV $\left.{ }^{+} 10\right]$ Jonas Poelmans, Guido Dedene, Gerda Verheyden, Herman Van der Mussele, Stijn Viaene, and Edward Peters. Combining business process and data discovery techniques for analyzing and improving Integrated Care Pathways. In Petra Perner, editor, ICDM, volume 6171 of Lecture Notes in Computer Science, pages 505-517. Springer, 2010. 62

[PEVD09] Jonas Poelmans, Paul Elzinga, Stijn Viaene, and Guido Dedene. A case of using Formal Concept Analysis in combination with Emergent Self Organizing Maps for detecting domestic violence. In Petra Perner, editor, ICDM, volume 5633 of Lecture Notes in Computer Science, pages 247-260. Springer, 2009. 62

[Poe10] Jonas Poelmans. Essays on using Formal Concept Analysis in Information Engineering. PhD thesis, Faculteit Economie en Bedrijfswetenschappen, Katholieke Universiteit Leuven, 2010. 62

[Sch82] B. Schmidt. Ein Zusammenhang zwischen Graphentheorie und Verbandstheorie. Diplomarbeit, TH Darmstadt, 1982. 16, 26

[Sch87] Dieter Schütt. Abschätzungen für die Anzahl der Begriffe von Kontexten. Master's thesis, TH Darmstadt, 1987. 17, 115

[SKSW95] Rudi Schmiede, W. Kollewe, C. Sander, and R. Wille. TOSCANA als Instrument der bibliothekarischen Sacherschließung, volume 1716 of Preprint. Techn. Hochschule, FB Mathematik, Darmstadt, January 1995. Ersch. ebenf. in: Aufbau u. Erschließung begrifflicher Datenbanken. Hrsg.: H. Havekost u.a. - Oldenburg 1995. S. 95-114. 61 
[SSV ${ }^{+}$93] Patrick Scheich, Martin Skorsky, Frank Vogt, Wachter Cornelia, and Rudolf Wille. Conceptual data systems. Information and classification, pages 72-84, 1993. 61

[Stu00] Gerd Stumme. Conceptual on-line analytical processing. In Katsumi Tanaka, Shahram Ghandeharizadeh, and Yahiko Kambayashi, editors, Information organization and databases, chapter Conceptual on-line analytical processing, pages 191-203. Kluwer Academic Publishers, Norwell, MA, USA, 2000. 62

[Tos11] ToscanaJ, 2011. http://toscanaj.sourceforge.net. 51

[Tut61] W. T. Tutte. On the problem of decomposing a graph in n connected factors. Journal of London Mathemathical Society, 36, 1961. 108

[Val79a] Leslie G. Valiant. The complexity of computing the permanent. Theoretical Computer Science, 8:189-201, 1979. 45

[Val79b] Leslie G. Valiant. The complexity of enumeration and reliability problems. SIAM Journal on Computing, 8(3):410-421, 1979. 45, 46

[Vog95] Niko Vogel. Ein begriffliches Erkundungssystem für Rohrleitungen. Master's thesis, TH Darmstadt, 1995. 61

[Web11] WebMotors, 2011. http://www.webmotors.com.br. 51

[Wil82] R. Wille. Restructuring lattice theory: an approach based on hierarchies of concepts. In Rival, I. (ed.): Ordered Sets, pages 445-470. Boston, 1982. 15, 16, 36,120

[Zsc07] Christian Zschalig. An FDP-algorithm for drawing lattices. In Peter W. Eklund, Jean Diatta, and Michel Liquiere, editors, CLA, volume 331 of CEUR Workshop Proceedings. CEUR-WS.org, 2007. 138 